\title{
Characterization of optically active perovskite electrodes
}

\author{
Gergely Ferenc Samu
}

Supervisors:

Dr. Csaba Janáky

Assistant professor

Prof. Csaba Visy

Professor emeritus

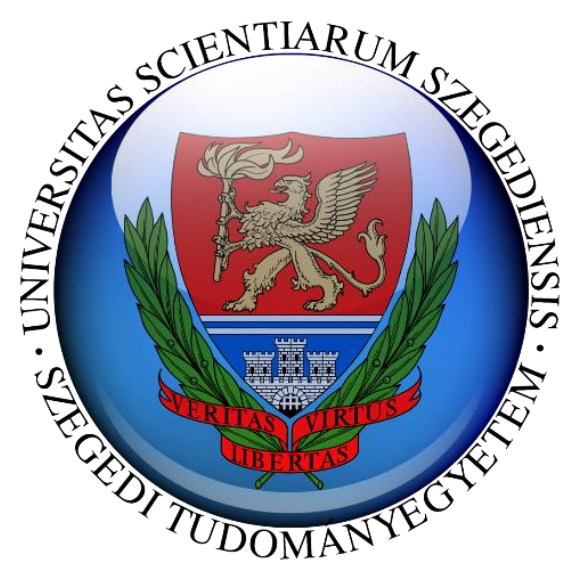

DOCTORAL SCHOOL OF CHEMISTRY

University of Szeged

Faculty of Science and Informatics

Department of Physical Chemistry and Materials Science

Szeged

2018 


\section{Table of contents}

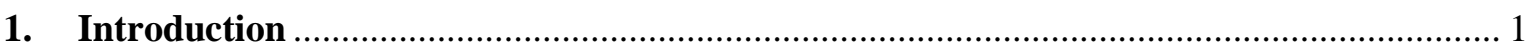

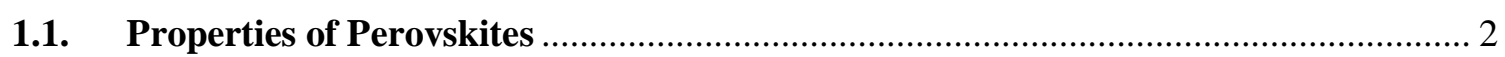

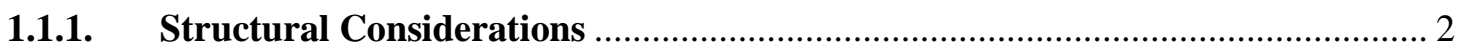

1.1.2. Synthetic Strategies to Prepare Perovskites............................................................ 5

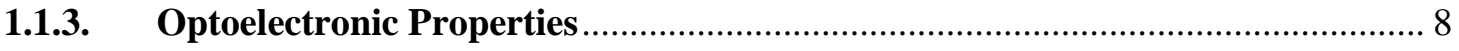

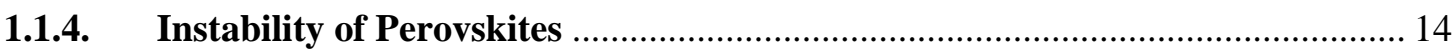

1.1.5. Light-Induced Phase Segregation in Mixed Halide Perovskites ....................... 20

1.1.6. Electrochemical Properties of Perovskites ......................................................... 20

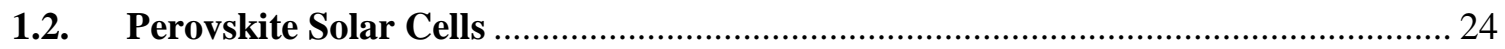

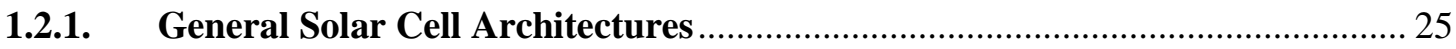

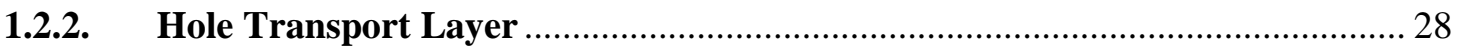

1.3. Conducting Polymers as Hole Transporters in Perovskite Solar Cells ..................... 29

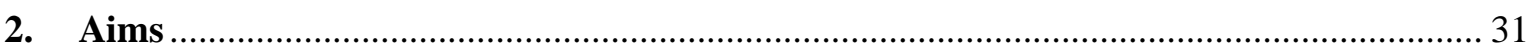

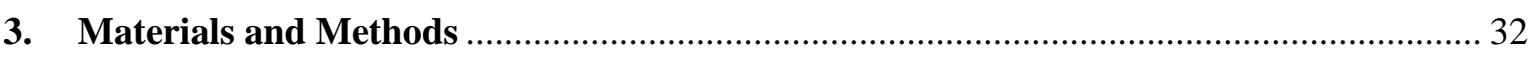

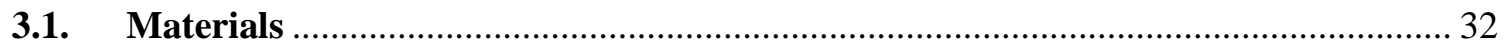

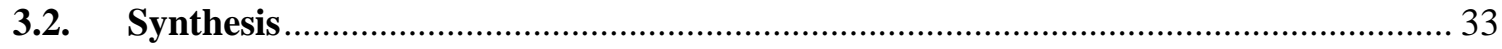

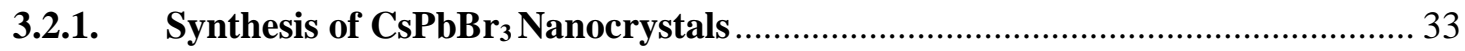

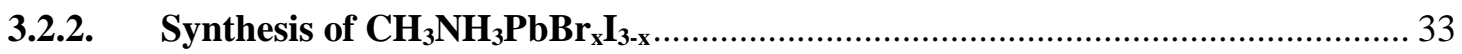

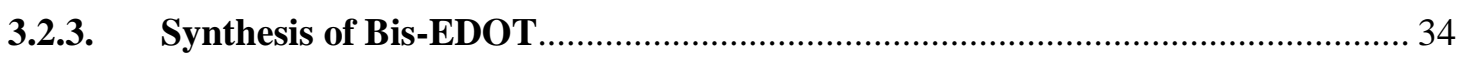

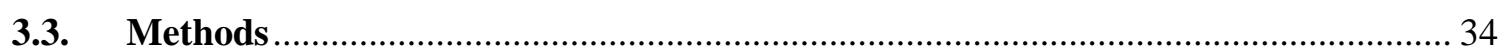

3.3.1. Electrode/Solar Cell Preparation ....................................................................... 34

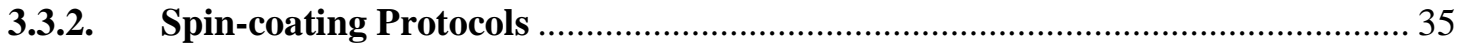

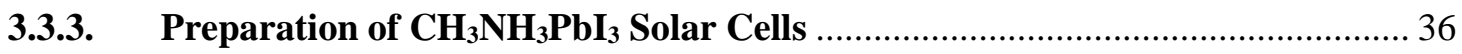

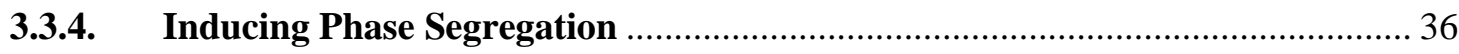

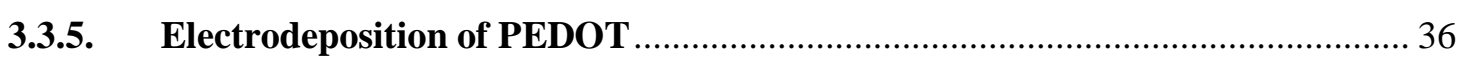

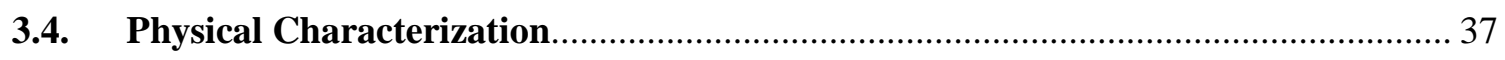

3.4.1. Electrochemistry/Spectroelectrochemistry …................................................. 37

3.4.2. Ultrafast Transient Spectroelectrochemistry ………........................................... 39

3.4.3. X-ray Photoelectron Spectroscopy .................................................................. 42

3.4.4. Scanning Electron Microscopy ...................................................................... 42

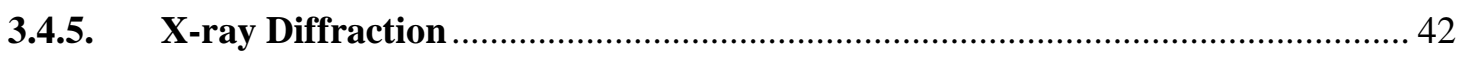

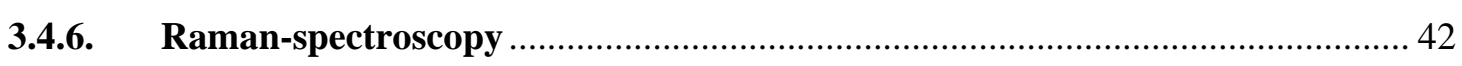

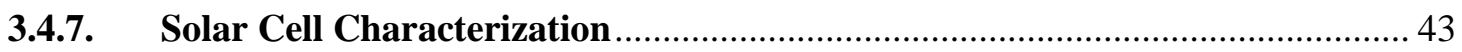

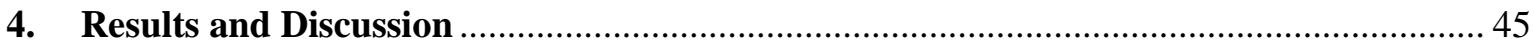

4.1. Establishing the Electrochemical Stability Window of Perovskite Electrodes ......... 45 
4.1.1. UV-vis Spectroscopic Studies of the Dissolution of $\mathrm{CsPbBr}_{3}$ Electrodes........... 45

4.1.2. Spectroelectrochemistry of $\mathrm{CsPbBr}_{3}$ Electrodes ................................................. 46

4.1.3. Chemical Composition of Reduced/Oxidized $\mathrm{CsPbBr}_{3}$ Electrode Surfaces...... 48

4.1.4. Structural Changes of Reduced/Oxidized $\mathrm{CsPbBr}_{3}$ Electrodes ......................... 51

4.1.5. Morphological Changes of Reduced/Oxidized $\mathrm{CsPbBr}_{3}$ Electrodes .................. 54

4.1.6. Spectroelectrochemistry of $\mathbf{P b B r}_{2}$ Electrodes................................................... 55

4.1.7. UV-vis Spectroscopic Studies of the Dissolution of $\mathrm{CH}_{3} \mathrm{NH}_{3} \mathrm{PbI}_{3}$ Electrodes ... 56

4.1.8. Spectroelectrochemistry of $\mathrm{CH}_{3} \mathrm{NH}_{3} \mathrm{PbI}_{3}$ Electrodes …...................................5

4.1.9. Spectroelectrochemistry of $\mathbf{P b I}_{2}$ Electrodes..................................................... 59

4.1.10. Potential Diagram of Optically Active Perovskite Electrodes............................ 60

4.1.11. Best Practice for Electrochemical Experiments ................................................ 61

4.2. Ultrafast Transient Spectroelectrochemistry of Perovskite Photoelectrodes ........... 63

4.2.1. Transient Absorption of $\mathrm{CsPbBr}_{3}$ with Applied Bias …..................................... 63

4.2.2. Effect of Electrochemical Bias on the Charge Carrier Recombination ............ 65

4.2.3. Reversibility of Bleaching Recovery Kinetics to Applied Bias .............................66 66

4.2.4. The Influence of $\mathrm{TiO}_{2}$ Layer on Bleaching Recovery Lifetime .......................... 69

4.3. Light-Induced Phase Segregation in Mixed Halide Perovskite Solar Cells .............. 71

4.3.1. UV-vis Spectroscopic Monitoring of Phase Segregation in $\mathrm{CH}_{3} \mathrm{NH}_{3} \mathrm{PbBr}_{1.5} \mathrm{I}_{1.5}$

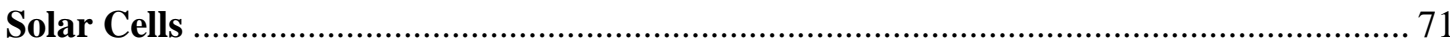

4.3.2. Effect of Phase Segregation on the Efficiency of $\mathrm{CH}_{3} \mathrm{NH}_{3} \mathrm{PbBr}_{1.5} \mathrm{I}_{1.5}$ Solar Cells . 72

4.3.3. Effect of Phase Segregation on the Quantum Efficiency of Solar Cells ............ 72

4.4. Electrosynthesis of PEDOT Hole Transporter Layer on $\mathbf{C H}_{3} \mathbf{N H}_{3} \mathbf{P b I}_{3}$ Electrodes .. 76

4.4.1. Spectroelectrochemistry of PEDOT Deposition on $\mathrm{CH}_{3} \mathbf{N H}_{3} \mathrm{PbI}_{3}$ Electrodes ... 76

4.4.2. Morphological Features of Electrodeposited PEDOT …................................... 78

4.4.3. Efficiency of Solar Cells Employing Electrodeposited PEDOT Hole Transporter

4.4.4. Electrochemical Post Treatment of the Deposited PEDOT Hole Transporter. 80

4.4.5. Fine-tuning the Electrochemical Post Treatment .............................................. 83

4.4.6. Transient Absorption Spectroscopy of Electrodeposited PEDOT Hole

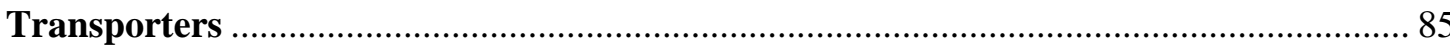

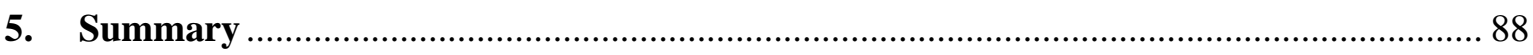

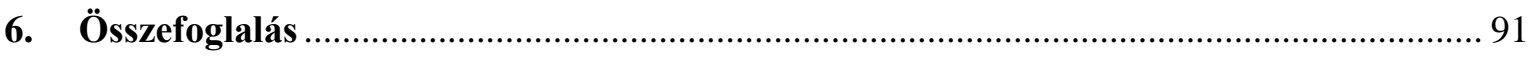

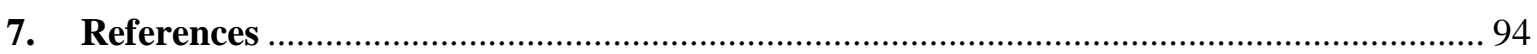

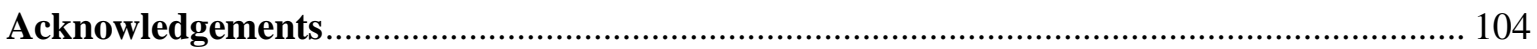




\section{List of Figures}

Figure 1. The improvement in power conversion efficiency of perovskite solar cells over the years, graphed together with the number of publications and the number of generated citations (Web of Knowledge statistics 2018.01.01 search term: perovskite solar cell)...... 1

Figure 2. Crystal structure of a $3 D$ HOIP, with a general stoichiometry of $A B X_{3}$............. 3

Figure 3. Formation landscape of $3 D$ lead (red line) and tin (blue line) perovskites as a function of A-site cation and X-site anion size. Solid and dashed lines mark the bounds of tolerance and octahedral factors respectively. Adapted from ref. ${ }^{[19]}$

Figure 4. HOIP thin film deposition methods. A: Antisolvent assisted spin-coating method. B: Two-step (sequential) deposition spin-coating method. C: Vapor deposition (coevaporation) method. Adopted from ref. ${ }^{[26]}$

Figure 5. Summary of optoelectronic properties of HOIPs.

Figure 6. A: $U V$-vis absorption spectra of MAPbBr${ }_{x} I_{3-x}$ thin layers. The inset shows the determined optical bandgap from the onset of the absorption spectra. B: Representation of the electronic band diagram of $\mathrm{MAPbBr}_{x} \mathrm{I}_{3-x}$ compounds.

Figure 7. Schematic representation of the various photophysical processes in perovskites following photoexcitation. Adapted from ref. ${ }^{[90]}$.

Figure 8. Important factors governing the stability HOIPs.

Figure 9. Illustration of the different migration pathways in a $\mathrm{MAPbI}_{3}$ lattice. A: The migration of $\mathrm{I}^{-}, \mathrm{Pb}^{2+}$, and $\mathrm{MA}^{+}$ions through vacancies. B: Different migration pathways considering bulk-point defects, lattice distortions, and grain boundaries. Adapted from ref. [134]

Figure 10. The evolution of PSC architectures. Adapted from ref. ${ }^{[176]}$. 25

Figure 11. Skeletal structural formula of commonly employed organic hole transporter materials.

Figure 12. Schematic representation of the different architectures used throughout the experiments. A: Complete solar cell, B: partially complete solar cell and $\boldsymbol{C}$ : electrode... 34

Figure 13. A: Photograph of a fully assembled $\mathrm{MAPbI}_{3}$ solar cell. B: The evolution of $M A P b I_{3}$ solar cell efficiencies throughout the course of the work.

Figure 14. Schematic representation of the spectroelectrochemical setup, together with the photograph of the cell.

Figure 15. The used measurement setup for the ultrafast transient spectroelectrochemistry measurements.

Figure 16. A: Energy levels of a hypothetical optically active sample and some quantum transitions influencing the difference absorption spectrum. B: The corresponding difference absorption spectrum with the separate contributions of the different transitions.

Figure 17. A: Typical $j$-V response of a MAPbI solar cell under dark, reverse and forward sweep conditions. The sweep rate was $50 \mathrm{mV} \mathrm{s}^{-1}$ and the used illumination was $1.5 \mathrm{AM} 100$ $m \mathrm{~W} \mathrm{~cm}^{-2}$. B: The equivalent circuit model of a realistic solar cell. 
Figure 18. $U V$-vis absorbance spectra of $\mathrm{FTO} / \mathrm{TiO}_{2} / \mathrm{Cs} \mathrm{PbBr}_{3}$ films in different electrolytes, after different immersion times. A: pure DCM, B: $0.1 M B u_{4} N B F_{4}$ in DCM, C: $0.1 M$ $\mathrm{Bu}_{4} \mathrm{NClO}_{4}$ in DCM, D: $0.1 \mathrm{M} \mathrm{Bu}_{4} \mathrm{NPF}_{6}$ in DCM.

Figure 19. Normalized absorbance change at $518 \mathrm{~nm}$ of $\mathrm{FTO} / \mathrm{TiO}_{2} / \mathrm{Cs}_{\mathrm{PbBr}}$ electrodes in different DCM based electrolytes. The error bars were derived from measurements on three separate films..... 46

Figure 20. Spectroelectrochemical data, recorded for $\mathrm{FTO} / \mathrm{TiO}_{2} / \mathrm{CsPbBr}_{3}$ films in $0.1 \mathrm{M}$ $B_{4} N_{N F} F_{6} / D C M$ electrolyte $\left(10 \mathrm{mV} \mathrm{s}^{-1}\right.$ sweep rate). A: Three subsequent $C V$ curves recorded during oxidation $\boldsymbol{B}: U V$-vis spectra recorded at various potentials during oxidation. $C:$ The first oxidation half cycle plotted together, with the absorbance change at the excitonic peak.

Figure 21. A: UV-vis absorbance spectrum of a $\mathrm{FTO}_{\mathrm{TiO}} / \mathrm{Cs}_{\mathrm{SbBr}}$ film in $0.1 \mathrm{M} \mathrm{Bu} 4 \mathrm{NPF}$. Difference spectra after $\boldsymbol{B}$ : different immersion times and $\boldsymbol{C}$ : oxidation treatment. 47

Figure 22. Spectroelectrochemical data, recorded for $\mathrm{FTO} / \mathrm{TiO}_{2} / \mathrm{CsPbBr}_{3}$ films in $0.1 \mathrm{M}$ $B u_{4} N P F_{6} / D C M$ electrolyte $\left(10 \mathrm{mV} \mathrm{s}^{-1}\right.$ sweep rate). A: Three subsequent $C V$ curves recorded during reduction $\boldsymbol{B}: U V$-vis spectra recorded at various potentials during reduction. $C:$ The reduction half cycle plotted together, with the absorbance change at excitonic peak. 48

Figure 23. Photographs of $\mathrm{FTO} / \mathrm{TiO}_{2} / \mathrm{Cs}_{\mathrm{PbBr}}$ electrodes treated at selected potential values for $30 \mathrm{~s}$.

Figure 24. High resolution XPS data for the $\mathrm{FTO} / \mathrm{TiO}_{2} / \mathrm{Cs}_{\mathrm{PbBr}}$ electrodes treated at selected potential values (as-is, $-1.4 \mathrm{~V},+0.8 \mathrm{~V},+1.5 \mathrm{~V}$ ), for $30 \mathrm{~s}$ in $0.1 \mathrm{M} \mathrm{Bu} \mathrm{APF}_{6} / \mathrm{DCM}$ solution. A: Cs $3 d, \boldsymbol{B}: \mathrm{Pb} 4 f, \boldsymbol{C}: \mathrm{Br} 3 d, \boldsymbol{D}: \mathrm{F} 1 \mathrm{~s}, \boldsymbol{E}: \mathrm{O} 1 \mathrm{~s} X \mathrm{P}$ spectrum. 49

Figure 25. A: XRD patterns for the $\mathrm{FTO} / \mathrm{TiO}_{2} / \mathrm{CsPbBr}_{3}$ electrodes treated at selected potential values (as is, $-1.4 \mathrm{~V},+0.8 \mathrm{~V},+1.5 \mathrm{~V}$ ) for $30 \mathrm{~s}$ in $0.1 \mathrm{M} \mathrm{Bu} 4 \mathrm{NPF} / \mathrm{DCM}$ solution. The peaks marked with \# represent overlapping diffractions of $\mathrm{CsPbBr}_{3}$ and the $\mathrm{FTO}_{\mathrm{TiO}}$ substrate. B: Magnified regions of the diffraction patterns of the samples held at $-1.4 \mathrm{~V}$ and $+0.8 \mathrm{~V}$. The peaks marked with * belong to the untreated $\mathrm{FTO} / \mathrm{TiO}_{2} / \mathrm{Cs} \mathrm{PbBr} 3$ electrodes. 51

Figure 26. High resolution XPS data for the $\mathrm{FTO}_{\mathrm{TiO}} / \mathrm{Cs}_{\mathrm{PbBr}}$ electrodes, treated at selected potential values $(O C P,-0.4 \mathrm{~V}$, and $+0.4 \mathrm{~V})$ for $30 \mathrm{~s}$ in $0.1 \mathrm{M} \mathrm{Bu}{ }_{4} N P F_{6} / D C M$ solution. A: $C s$ 3d, B: $\mathrm{Pb} 4 f, \boldsymbol{C}: \mathrm{Br} 3 d, \boldsymbol{D}: \mathrm{F} 1 \mathrm{~s}, \boldsymbol{E}: \mathrm{O}$ 1s XP spectrum.

Figure 27. SEM images for the $\mathrm{FTO} / \mathrm{TiO}_{2} / \mathrm{Cs} \mathrm{PbBr}_{3}$ electrodes treated at selected potential values $(A: O C P, B:+0.4 \mathrm{~V}$, and $\boldsymbol{C}:-0.4 \mathrm{~V})$ for $30 \mathrm{~s}$ in $0.1 \mathrm{M} \mathrm{Bu} \mathrm{NPF}_{6} / D C M$ solution.... 54

Figure 28. SEM images of $\mathrm{Cs} \mathrm{PbBr}_{3}$ films both from top and side views. $\boldsymbol{A}, \boldsymbol{E}$ : pristine, $\boldsymbol{B}, \boldsymbol{F}$ : reduced at $E=-1.4 \mathrm{~V}, \boldsymbol{C}, \boldsymbol{G}:$ oxidized at $E=+0.8 \mathrm{~V}, \boldsymbol{D}, \boldsymbol{H}$ : oxidized at $E=+1.4 \mathrm{~V}$. All treatments lasted $30 \mathrm{~s}$ in $0.1 \mathrm{M} \mathrm{Bu} \mathrm{NPF}_{6} / \mathrm{DCM}$ solution. 55

Figure 29. SEM image and EDX data recorded for a $\mathrm{FTO} / \mathrm{TiO}_{2} / \mathrm{Cs} \mathrm{PbBr}_{3}$ electrode treated at $E=-1.4 \mathrm{~V}$ for $30 \mathrm{~s}$ in $0.1 \mathrm{MB} \mathrm{Bu}_{4} N \mathrm{PF} / \mathrm{DCM}$ solution.

Figure 30. Three subsequent $C V$ curves recorded in $0.1 B_{4} N^{2} F_{6} / D C M$ solution $\left(10 \mathrm{mV} \mathrm{s}^{-1}\right.$ sweep rate), during the $\mathrm{A}$ : oxidation and $\boldsymbol{B}$ : reduction of the $\mathrm{PbBr}_{2}$ films deposited on $\mathrm{FTO}_{\mathrm{TiO}}$ electrodes. $U V$-vis spectra recorded during $\mathrm{C}$ : oxidation and $\mathrm{D}$ : reduction processes. 
Figure 31. UV-vis absorbance spectra of $\mathrm{FTO} / \mathrm{TiO}_{2} / \mathrm{MAPbI}_{3}$ films in different electrolytes, after different immersion times. A: pure DCM, B: $0.1 M B u_{4} N B F_{4}$ in DCM, C: $0.1 M$ $\mathrm{Bu}_{4} \mathrm{NClO}_{4}$ in DCM, D: $0.1 \mathrm{M} \mathrm{Bu}_{4} \mathrm{NPF}_{6}$ in DCM, E: $0.01 \mathrm{M} \mathrm{Bu} \mathrm{NPF}_{6}$ in DCM.

Figure 32. Kinetic curves of the normalized absorbance change at $470 \mathrm{~nm}$, recorded for $\mathrm{FTO} / \mathrm{TiO} / \mathrm{MAPbI}_{3}$ in different DCM based electrolytes. The error bars were derived from the measurement of three separate films. 58

Figure 33. Spectroelectrochemical data, recorded for $\mathrm{FTO}_{\mathrm{TiO}} / \mathrm{MAPbI}_{3}$ films in $0.1 \mathrm{M}$ Bu $4{ }_{4} F_{6} / D C M$ solution $\left(10 \mathrm{mV} \mathrm{s}^{-1}\right.$ sweep rate), during the first A: oxidation and $\boldsymbol{B}$ : reduction half cycles together with the absorbance changes at $470 \mathrm{~nm}$. 58

Figure 34. Three subsequent $C V$ curves recorded during the $A$ : oxidation and $B$ : reduction of $\mathrm{FTO} / \mathrm{TiO}_{2} / \mathrm{MAPbI}_{3}$ electrodes in $0.01 \mathrm{Bu}_{4} \mathrm{NPF}_{6} / \mathrm{DCM}$ solution $\left(10 \mathrm{mV} \mathrm{s}^{-1}\right.$ sweep rate). $U V$-vis spectra recorded during the first $\boldsymbol{C}$ : oxidation and $\boldsymbol{D}$ : reduction half-cycle. 59

Figure 35. Three subsequent $C V$ curves recorded in $0.1 B_{4} N P F_{6} / D C M$ solution $\left(10 \mathrm{mV} \mathrm{s}^{-1}\right.$ sweep rate), during the $\mathrm{A}$ : oxidation and $\mathrm{B}$ : reduction of the $\mathrm{PbI}_{2}$ films deposited on $\mathrm{FTO}_{\mathrm{TiO}}$ electrodes. $U V$-vis spectra recorded during the first $\mathrm{C}$ : oxidation and $\mathrm{D}$ : reduction half-cycles.

Figure 36. Comparison of the band edge positions and the potential of various redox events detected for the studied two lead halide perovskites.

Figure 37. A: Time vs. applied potential curve recorded during electrochemical stability measurements. The potential was ramped from $-0.6 \mathrm{~V}$ to $+0.6 \mathrm{~V}$ and then back to $-0.6 \mathrm{~V}$. B: Time vs. the normalized absorbance at $518 \mathrm{~nm}$ (excitonic peak) of the $\mathrm{FTO} / \mathrm{TiO}_{2} / \mathrm{CsPbBr}_{3}$ film used for the electrochemical stability test. $C$ : Absorbance spectra of the $\mathrm{FTO} / \mathrm{TiO}_{2} / \mathrm{Cs} \mathrm{PbBr}_{3}$ film before and after the cycling of applied bias.

Figure 38. Difference absorption spectra of an $\mathrm{FTO} / \mathrm{TiO}_{2} / \mathrm{Cs} \mathrm{PbBr}_{3}$ film measured A: in vacuum (without electrolyte) and B: in DCM containing $0.1 \mathrm{M} \mathrm{Bu} \mathrm{APF}_{6}$ electrolyte (with electrolyte) under OCP conditions (no external bias was used). C: Respective bleaching recovery profiles recorded in the two different media. 64

Figure 39. Time resolved transient spectra recorded following $387 \mathrm{~nm}$ laser pulse excitation $\left(4 \mu \mathrm{J} \mathrm{cm}^{-2}\right)$ of $\mathrm{FTO} / \mathrm{TiO}_{2} / \mathrm{CsPbBr}_{3}$ electrode in a spectroelectrochemical cell with deaerated $D C M$ containing $0.1 M B u_{4} N P F_{6}$ electrolyte. The spectra were recorded at applied potentials of $\boldsymbol{A}:+0.4 \mathrm{~V} ; \boldsymbol{B}:-0.4 \mathrm{~V} ; \boldsymbol{C}:+1.0 \mathrm{~V}$ and $\mathrm{D}:-1.2 \mathrm{~V} v \mathrm{~s} . \mathrm{Ag} / \mathrm{AgCl}$.

Figure 40. A: Bleaching recovery profiles, B: kinetic fit $1 / \Delta A$ vs. time and $\boldsymbol{C}$ : relative pseudo first order rate constants determined from the slope of plots in $\boldsymbol{B}$ with the dashed line to guide the eye (The magnitude of bleaching was kept constant at $\Delta A=-0.25$ for all these experiments so that initial charge carrier concentration is same in all experiments). The applied potentials range was between -0.6 and $+0.6 \mathrm{Vvs} . \mathrm{Ag} / \mathrm{AgCl}$. 66

Figure 41. Dependence of bleaching recovery lifetime determined from the inverse slope of the kinetic fit of $1 / \triangle A$ vs. time. Measurements were carried out with $\mathrm{FTO}_{\mathrm{TiO}} / \mathrm{CsPbBr}_{3}$ electrodes in deaerated DCM containing $0.1 \mathrm{M} B u_{4} N P F_{6}$ electrolyte. The electrodes were biased at set potentials in the range $-0.6 \mathrm{~V}$ to $+0.5 \mathrm{Vvs} . \mathrm{Ag} / \mathrm{AgCl}$ and excited with $387 \mathrm{~nm}$ laser pulse to record time resolved TA spectra. 
Figure 42. Difference absorption spectra of an $\mathrm{FTO} / \mathrm{TiO}_{2} / \mathrm{Cs}_{\mathrm{PbBr}}$ film at different applied potentials on a forward and reverse scan. Spectra are shown for A: -0.4 V forward; B: 0.0 $V$ forward; $\boldsymbol{C}:+0.4 V$ forward; $\boldsymbol{D}:-0.4 V$ reverse; $\boldsymbol{E}: 0.0$ V reverse; $\boldsymbol{F}:+0.4 V$ reverse. The same film was used for all measurements seen here. However, the film was moved to different positions so that degradation due to the pump laser pulse would not occur. 68

Figure 43. Dependence of bleaching recovery lifetime determined from the inverse slope of the kinetic fit of $1 / \triangle A$ vs. time. Measurements were carried out with FTO/CsPbBr 3 electrodes in deaerated DCM containing $0.1 \mathrm{MB} \mathrm{Bu}_{4} \mathrm{NPF}$ electrolyte. The electrodes were biased at set potentials in the range $-0.4 \mathrm{~V}$ to $+0.5 \mathrm{Vvs} . \mathrm{Ag} / \mathrm{AgCl}$ and excited with $387 \mathrm{~nm}$ laser pulse to record time resolved $T A$ spectra.

Figure 44. Schematic diagram illustrating the band energies and the Fermi level response to applied bias. The charge transfer from excited perovskite to $\mathrm{TiO}_{2}\left(k_{E T}\right)$ competes with the charge recombination $\left(k_{r}\right)$ is influenced by the electron accumulation (or depletion) during electrochemical bias

Figure 45. A: Absorption spectra of mixed halide perovskite film (a) before and (b) after irradiation for 30 minutes. Spectrum (c) shows dark recovery after three hours. B: Difference absorption spectra after 30 min light soaking shows dark recovery...... 71

Figure 46. A: $j$-V curves of the champion cell (a) in dark, (b) before light soaking, and (c) after light soaking for 30 minutes. B: Effect of 15 minute and 30 minute light soaking on the performance of four MAPBBr ${ }_{1.5} I_{1.5}$ perovskite based cells. The measured photoconversion efficiency before and after light soaking presented in the figure show the effect of light soaking on the photovoltaic performance.

Figure 47. A: IPCE of mixed halide PSC (a) before light soaking and $(b)-(f)$ during dark recovery following $30 \mathrm{~min}$ light soaking. A $420 \mathrm{~nm}$ cut-off filter was used for IPCE measurements and residual dark currents were corrected for IPCE measurements. B: Difference IPCE spectra showing the recovery of the solar cell response in the 500-700 $\mathrm{nm}$ region. (The spectra were normalized at $470 \mathrm{~nm}$ and the IPCE recorded before the continuous irradiation was used as the reference.). 73

Figure 48. Recovery traces recorded after light soaking of MAPbBr I.5 $_{1.5}$ based solar cells for 30 minutes (a) the recovery of the bleaching (observed at $620 \mathrm{~nm}$ ) using a partially assembled cell and $(b)$ the overall IPCE recovery of a complete cell.

Figure 49. Difference IPCE recorded for $\mathrm{MAPbI}_{3}$ based solar cell irradiated for 30 minutes (the IPCE data were normalized at $450 \mathrm{~nm}$ ). 74

Figure 50. Schematic representation of phase segregation in mixed-halide (MAPbBr $\left.{ }_{1.5} I_{1.5}\right)$ solar cells, and its effect on the charge collection of such devices.

Figure 51. A: Potentiodynamic deposition of PEDOT with $25 \mathrm{mV} \mathrm{s}^{-1}$ sweep rate in a 0.01 $M$ bis-EDOT, $0.1 \mathrm{M} \mathrm{Bu} \mathrm{NPF}_{6} \mathrm{DCM}$ solution on a solar cell architecture (FTO/bl-TiO $/ \mathrm{mp}$ $\mathrm{TiO}_{2} / \mathrm{MAPbI}_{3}$ ) employing a thin $\mathrm{MAPbI}_{3}$ layer. $\mathrm{B}: \mathrm{UV}$-vis absorbance spectra recorded after each cycle at $E=0.0 \mathrm{~V}$ during polymerization. $\boldsymbol{C}$ : First half-cycle of the potentiodynamic deposition plotted together with the absorbance change at $700 \mathrm{~nm}$.

Figure 52. A: Potentiostatic deposition of PEDOT at different potentials in a $0.01 \mathrm{M}$ bisEDOT, $0.1 \mathrm{M} \mathrm{Bu} 4 \mathrm{NPF}_{6} \mathrm{DCM}$ solution on a solar cell architecture employing regular 
thickness $\mathrm{MAPbI}_{3}$ layers with a polymerization charge density of $10 \mathrm{mC} \mathrm{cm}^{-2} . \mathbf{B}: U V$-vis absorbance spectra of solar cells after PEDOT electrodeposition at $E=+0.9 \mathrm{~V}$ for different polymerization charge densities. $C$ : The absorbance change of the solar cells compared to a HTM-free cell by varying the polymerization charge density at different applied potentials.

Figure 53. A: Top-view SEM image before (upper part) and after PEDOT (lower part) electrodeposition. B: Cross sectional FIB-SEM image of a solar cell, where the PEDOT electrodeposition was carried out at $E=+1.0 \mathrm{~V}$ with $Q_{p o l}=5 \mathrm{mC} \mathrm{cm}{ }^{-2} . C$ : Theoretical and actual PEDOT layer thicknesses (determined from cross-sectional SEM images). D: Ramanspectra of a fully assembled solar cell architecture and a FTO/PEDOT reference.

Figure 54. The effect of PEDOT thickness (polymerization charge density) in a FTO/TiO $/ \mathrm{MAPbI}_{3} / \mathrm{PEDOT}$ solar cell on the A: short circuit current density, B: open circuit voltage, $\boldsymbol{C}$ : fill factor, and D: overall efficiency. The solar cells were tested under 1 sun (100 $\mathrm{mW} \mathrm{cm}^{-2}$ ) illumination, with a $50 \mathrm{mV} \mathrm{s}^{-1}$ sweep rate.

Figure 55. The effect of PEDOT thickness (polymerization charge density) in a $\mathrm{FTO}_{\mathrm{TiO}} / \mathrm{MAPbI}_{3} / \mathrm{PEDOT}$ solar cell for PEDOT layers electrodeposited at $\mathrm{E}=+1.0 \mathrm{~V}$. A: on the open circuit voltage $\boldsymbol{B}$ : on the $j$-V profile of the solar cell in the dark $\left(50 \mathrm{mV} \mathrm{s}{ }^{-1}\right) .80$

Figure 56. Schematic representation of PEDOT doping.

Figure 57. Chronoamperometric curves recorded for the post-treatment of a $\mathrm{FTO}_{\mathrm{TiO}} / \mathrm{MAPbI}_{3} / \mathrm{PEDOT}$ solar cell, where the PEDOT layers were electrodeposited at $E$ $=+1.0 \mathrm{~V}, \boldsymbol{A}:$ when an oxidative post-treatment at $E=+0.6 \mathrm{~V}$ and $\boldsymbol{B}:$ when a reductive posttreatment at $E=-0.6 \mathrm{~V}$ was employed.

Figure 58. Development of the open circuit voltage in a FTO/TiO $/ \mathrm{MAPbI}_{3} / \mathrm{PEDOT}$ solar cell for the PEDOT layers electrodeposited at $E=+1.0 \mathrm{~V} \mathrm{for} Q_{p o l}=10 \mathrm{mC} \mathrm{cm}^{-2}$ as a function of redox treatment. 82

Figure 59. Cross-sectional SEM images of a solar cell, where the PEDOT electrodeposition

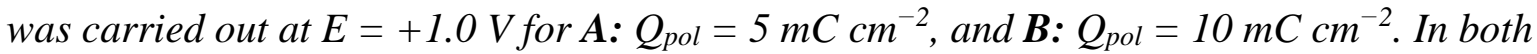
cases the electrodeposition was immediately followed by a reduction step at $E=-0.6 \mathrm{~V}$ for $t=60 \mathrm{~s}$.

Figure 60. Chronoamperometric curves recorded for the post-treatment of FTO/TiO $/ \mathrm{MAPbI}_{3} / \mathrm{PEDOT}$ solar cells, where the PEDOT layers were electrodeposited at $E=+1.0 \mathrm{~V}$. A reductive post treatment of A: rapid reduction at $E=-0.6 \mathrm{~V}$ for $t=10 \mathrm{~s}, \boldsymbol{B}$ : mild reduction at $E=-0.5 \mathrm{~V}$ for $t=60 \mathrm{~s}$, and $\mathrm{C}$ : rapid-mild reduction at $E=-0.5 \mathrm{~V}$ for $t$ $=20 \mathrm{~s}$ was used. 84

Figure 61. The effect of PEDOT thickness (polymerization charge density) on the open circuit voltage in a $\mathrm{FTO}_{\mathrm{TiO}} / \mathrm{MAPbI}_{3} / \mathrm{PEDOT}$ solar cell for PEDOT layers electrodeposited at $E=+1.0 \mathrm{~V}$. The applied post-reduction step was A: rapid reduction at $E=-0.6 \mathrm{~V}$ for $t=10 \mathrm{~s}, \boldsymbol{B}:$ mild reduction at $E=-0.5 \mathrm{~V}$ for $t=60 \mathrm{~s}$, and $\boldsymbol{C}:$ rapid-mild reduction at $E=-0.5$ for $t=20 \mathrm{~s}$. 84

Figure 62. $J$-V curve of the champion device containing a PEDOT layer electrodeposited at $E=+1.0 \mathrm{Vfor} Q_{p o l}=10 \mathrm{mC} \mathrm{cm}{ }^{-2}$, where the post-reduction step was at $E=-0.6 \mathrm{~V}$ for $t=$ $10 \mathrm{~s}$. 
Figure 63. A: Time-resolved transient spectra of an $\mathrm{FTO} / \mathrm{TiO}_{2} / \mathrm{MAPbI}_{3} / \mathrm{PEDOT}$ solar cell employing a thin $\mathrm{MAPbI}_{3}$ layer following $387 \mathrm{~nm}$ laser pulse excitation. The PEDOT layer was prepared and post-treated just as the champion device. B: Bleaching recovery profiles at $760 \mathrm{~nm}$ of different $\mathrm{FTO} / \mathrm{TiO}_{2} / \mathrm{MAPbI}_{3} / \mathrm{PEDOT}$ solar cells 86 


\section{List of Tables}

Table 1. Summary of the performance of the HOIPs used in Li-intercalation studies. In brackets the charging current density is shown. 23

Table 2. Drying conditions of the different conducting electrolytes. 38

Table 3. Compositional analysis of the surface of the electrochemically treated $\mathrm{FTO}_{\mathrm{TiO}} / \mathrm{Cs}_{\mathrm{PbBr}}$ electrodes using XPS. The standard deviation values were derived from analyzing XP spectra captured from three different spots of the sample. 50

Table 4. Compositional analysis of the surface of the $\mathrm{FTO} / \mathrm{TiO}_{2} / \mathrm{Cs}_{\mathrm{PbBr}}$ electrodes oxidized at selected potential values using XPS.

Table 5. Compositional analysis of the surface of the mildly treated $\mathrm{FTO} / \mathrm{TiO}_{2} / \mathrm{CsPbBr}_{3}$ electrodes using XPS. 53

Table 6. Parameters to conduct (spectro)electrochemical experiments. 62

Table 7. Determined lifetimes, from biexponential fits carried out on TA data. The standard deviation of the measurements was calculated from three separate measurements. 86 


\section{List of Abbreviations}

$\mathrm{CB}-$ Conduction band

$\mathrm{CV}-$ Cyclic voltammetry

DSSC - Dye-sensitized solar cell

ECL - Electrochemiluminescence

EDX - Energy dispersive X-ray spectroscopy

ETL - Electron transport layer

FIB - Focused ion beam

HOIP - Hybrid organic-inorganic perovskite

HTL / HTM - Hole transport layer / Hole transport material

IPCE - Incident photon to charge carrier conversion efficiency

$\mathrm{J}_{\mathrm{SC}}$ - Short-circuit current density

$\mathrm{V}_{\mathrm{OC}}-$ Open circuit potential

PSC - Perovskite solar cell

SEM - Scanning electron microscopy

TAS - Transient absorption spectroscopy

UV-vis - Ultraviolet-visible

VB - Valence band

$\mathrm{V}_{\mathrm{OC}}-$ Open-circuit voltage

XPS - X-ray photoelectron spectroscopy

XRD - X-ray diffraction

\section{Chemicals}

DCM - Dichloromethane

DMF - N,N-dimethylformamide

DMSO - Dimethylsulfoxide

FTO - Fluorine doped tin oxide

GBL $-\gamma$-butyrolactone

$\mathrm{MAPbX}_{3}-\mathrm{CH}_{3} \mathrm{NH}_{3} \mathrm{PbX}$

MAI - Methylammonium-iodide

PEDOT - Poly(3,4-ethylenedioxythiophene)

spiro-MeOTAD - 2,2',7,7'-tetrakis(N,N'-di-p-methoxyphenylamine)-9,9'-spirobifluorene 


\section{Introduction}

The emergence of hybrid organic-inorganic perovskites (HOIPs) as active components in solar cells has sparked a rapidly growing interest in the scientific community (Figure 1). A steep rise was witnessed in the power conversion efficiencies of perovskite solar cells (PSCs), which currently peaks at a certified value of $22.1 \% .^{[1]}$ Applications beyond solar cells have also been studied, in spintronics, ${ }^{[2]}$ light emitting diodes, ${ }^{[3,4]}$ lasers, ${ }^{[4]}$ photodetectors, ${ }^{[5-7]} \mathrm{X}$-ray detectors,${ }^{[8]}$ and gamma detectors. ${ }^{[9]}$

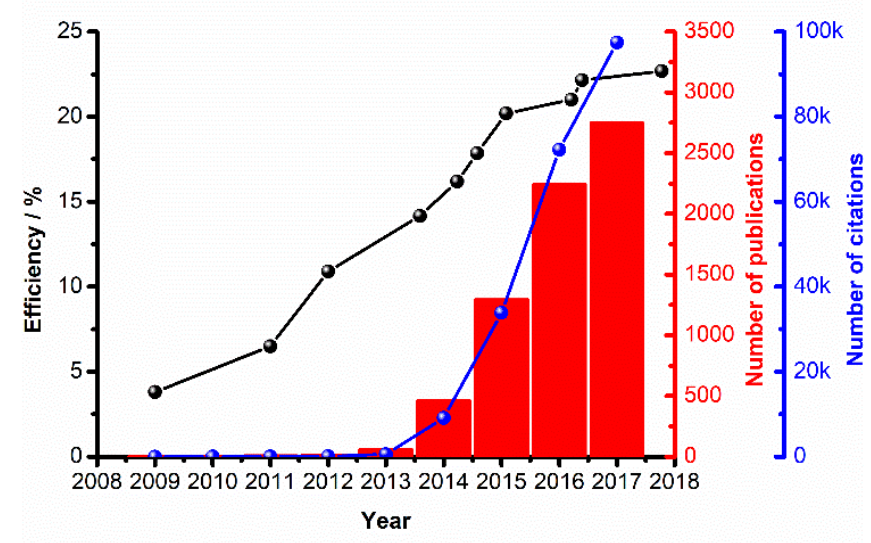

Figure 1. The improvement in power conversion efficiency of perovskite solar cells over the years, graphed together with the number of publications and the number of generated citations (Web of Knowledge statistics 2018.01.01 search term: perovskite solar cell).

HOIPs are extremely versatile as a large portion of elements (or even organic molecules) can be used as the building blocks of the perovskite lattice. The criteria that these elements must meet are mainly size constraints (i.e., Goldschmidt tolerance and octahedral factor). The interchangeability in composition is the reason behind the fact that several properties of HOIPs are exceptionally fine-tunable.

Simple solution processes can be used to prepare efficient HOIP based devices (e.g., one-step, two-step and vacuum assisted processes). Throughout the years several different methods (or improvements on an already existing one) aimed to gain better control over the crystallization process of HOIPs. The optoelectronic properties of HOIPs show extreme tunability and versatility. Their large absorption coefficient and composition dependent bandgap makes them ideal materials for light absorbing and emitting applications. These materials are mainly non-excitonic in nature (low exciton binding energy), which ensures efficient charge carrier generation. Furthermore, their defect tolerant crystal structure (e.g., dominance of shallow point defects and the absence of impurity states) suppresses charge 
carrier recombination processes and their ambipolar charge transport improves charge carrier extraction. These qualities are the underlying reasons for the remarkable efficiency of the derived solar cells.

Currently the limiting factor of the commercialization of HOIP based devices is their sensitivity towards several environmental factors (e.g., humidity, heat, light). Furthermore, device architecture, operational conditions (such as built-in electric fields) and other components of the devices (interfacial layers, electrode corrosion) can also detrimentally affect device performance.

Electrochemistry as a characterization tool, has started to gain a foothold in the evaluation of HOIP materials. Currently the field is in its infancy, however the first studies are already available of HOIP based electrodes in different electrochemical applications (e.g., Li-ion batteries, electrochemiluminescence). Electrochemical techniques can offer valuable information about the charge carrier generation and transport in HOIPs. With the aid of coupled techniques the chemical changes upon charge carrier generation can also be unraveled. Fundamental optoelectronic properties can also be studied (e.g., band edge positions, distribution of trap states). In addition, electrochemical methods can be also used as synthetic tools. The use of HOIP photoelectrodes to drive certain reduction reactions (e.g., $\mathrm{CO}_{2}$ reduction) is an especially lucrative concept. Furthermore, electrochemistry can also aid the assembly of HOIP based devices. All these opportunities call for coherent stability tests, so the degradation of the HOIP based electrodes could be avoided.

\subsection{Properties of Perovskites}

\subsubsection{Structural Considerations}

Perovskites constitute an intriguing class of materials, which have played a pivotal role in the evolution of materials chemistry and condensed matter physics. The crystallographic family itself bears the name of the Russian mineralogist Lev A. Perovski. However, the first perovskite compound $\left(\mathrm{CaTiO}_{3}\right)$, was discovered by Gustav Rose in 1839 . These materials adopt a general stoichiometry of $\mathrm{ABX}_{3}$. Initially oxide compounds dominated this crystallographic family. In recent years the adaptability of the perovskite structure to site substitution gave rise to a new class of perovskites, namely HOIPs. Normally, the A site can be occupied by a monovalent organic cation (e.g., methylammonium $\left(\mathrm{CH}_{3} \mathrm{NH}_{3}{ }^{+}, \mathrm{MA}^{+}\right)$, formamidinium $\left(\mathrm{CH}\left(\mathrm{NH}_{2}\right)_{2}{ }^{+}, \mathrm{FA}\right)$ or inorganic cation (e.g., $\mathrm{K}^{+}, \mathrm{Rb}^{+}, \mathrm{Cs}^{+}$). The $\mathrm{B}$ site is a divalent metal cation (e.g., $\mathrm{Pb}^{2+}, \mathrm{Sn}^{2+}, \mathrm{Ge}^{2+}$ ) and the $\mathrm{X}$ 
site is occupied by halide anions $\left(\mathrm{F}^{-}, \mathrm{Cl}^{-}, \mathrm{Br}^{-}, \mathrm{I}^{-}\right)$, albeit pseudohalides $\left(\mathrm{SCN}^{-}\right)$and complex anions (e.g., $\mathrm{BF}_{4}^{-}, \mathrm{PF}_{6}^{-}$) can also be considered. The 3 dimensional perovskite lattice (Figure 2) is formed by corner-connected $\mathrm{BX}_{6}$ octahedrons, and A cations that fill up the 12-fold cuboctahedral voids.

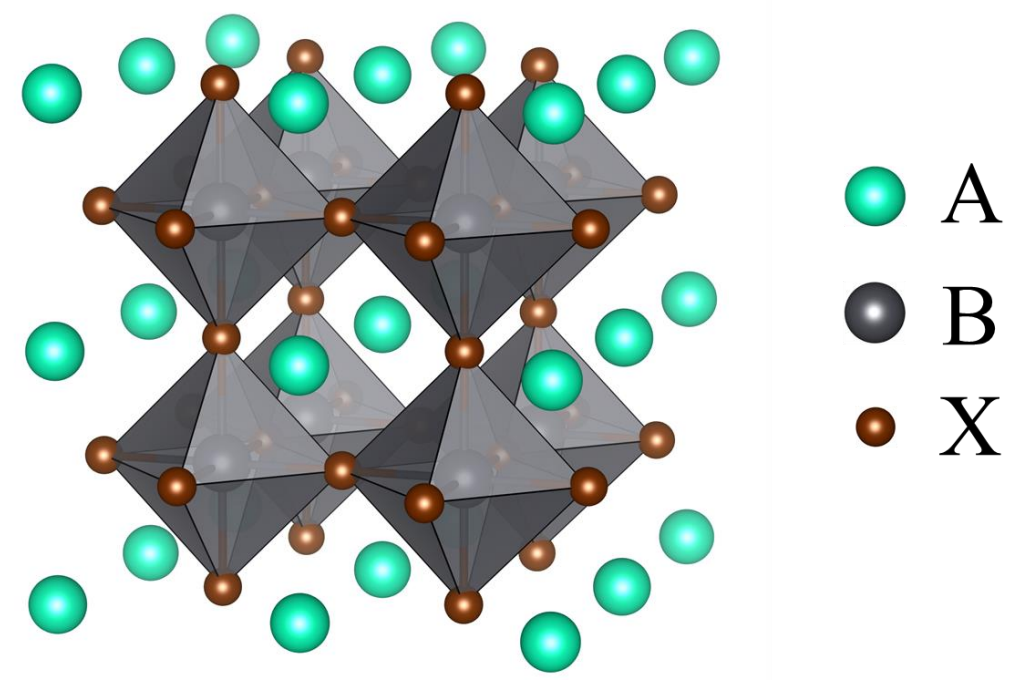

Figure 2. Crystal structure of a $3 D$ HOIP, with a general stoichiometry of $A B X_{3}$.

Although a multitude of elements can be incorporated into the perovskite framework, there are several criteria that these elements must fulfill. The most important geometric constraint is that the A cation must fit into the cuboctahedral voids formed by the $\mathrm{BX}_{6}$ octahedrons. A semiempirical geometric parameter, the Goldschmidt tolerance factor $(\mathrm{t}),{ }^{[10]}$ can be used to predict the stability of a $3 D$ perovskite structure, where $r_{A}, r_{B}$ and $r_{X}$ are the ionic radii of the $\mathrm{A}, \mathrm{B}$ cation and $\mathrm{X}$ anion respectively:

$$
t=\frac{r_{A}+r_{X}}{\sqrt{2}\left(r_{B}+r_{X}\right)}
$$

For most 3D perovskites the Goldschmidt tolerance factor falls in the range of $0.8 \leq \mathrm{t} \leq 1.0$. The lower this value is, the ideal cubic perovskite lattice becomes more distorted by the gradual tilting of the $\mathrm{BX}_{6}$ octahedral units in the lattice. ${ }^{[11]}$ As a result, if $0.8 \leq \mathrm{t} \leq 0.9$, the appearance of orthorhombic, tetragonal and rhombohedral perovskite lattices should be expected. Below $\mathrm{t}=0.8$, other crystal structures (e.g., ilmenite-type) become more stable. Interestingly, in the case of lower dimensional perovskites, the structure can accommodate larger A site cations than the Goldschmidt tolerance factor would suggest $(t>1) .{ }^{[12-14]}$ Note that lower dimensionality refers to the degree of connectivity of the $\mathrm{BX}_{6}$ octahedrons. These 
lower dimensional perovskites, are specific cuts or slices of the parent 3D perovskite structure.

The Goldschmidt tolerance factor is an often-used tool to predict possible material compositions that could form a perovskite lattice. It has been shown that it is a necessary but not sufficient condition as other space filling restrictions should also be considered. ${ }^{[15,16]}$ One such is the stability of the $\mathrm{BX}_{6}$ octahedron, which can be described by the octahedral factor $(\mu)$ :

$$
\mu=\frac{r_{B}}{r_{X}}
$$

The formation of a perovskite lattice is expected if the octahedral factor falls in the range of $0.442 \leq \mu \leq 0.895 .^{[15,16]}$ From these two threshold conditions a simple map for perovskite formation can be constructed (Figure 3). These calculations assume that the ions are rigid spheres and that a close packing is achieved. These assumptions are straightforward in the case of inorganic ions, however asymmetric organic cations (where hydrogen-bonding can vary bond lengths) can pose a challenge. ${ }^{[17]}$ With these considerations recently more than 600 hypothetical organic-inorganic perovskites were identified. ${ }^{[18]}$

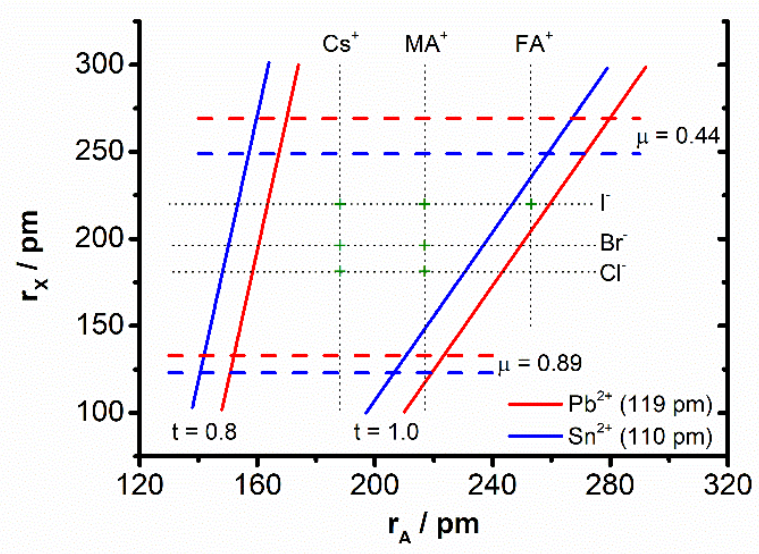

Figure 3. Formation landscape of $3 D$ lead (red line) and tin (blue line) perovskites as a function of A-site cation and X-site anion size. Solid and dashed lines mark the bounds of tolerance and octahedral factors respectively. Adapted from ref. ${ }^{[19]}$

The picture becomes even more complicated when the bonding/coordination preference of the metal ion is considered. ${ }^{[13]}$ In this sense three different phenomena can arise: 
- The lone $\mathrm{s}^{2}$ electron pair of the B site metal ion is stereochemically active, it can shift the metal ion away from the crystallographic center of the $\mathrm{BX}_{6}$ octahedron, thus causing a distortion in the perovskite framework. ${ }^{[20]}$ This effect is particularly dominant in the case of $\mathrm{Ge}^{2+}$ based perovskites and becomes less prominent in the case of $\mathrm{Pb}^{2+} \cdot{ }^{[21,22]}$

- In HOIPs the organic cation occupying the A site can also cause the distortion of the perovskite framework, through directional hydrogen bonding to the halide cage. ${ }^{[22]}$

- Jahn-Teller and related distortions can also affect the stability of the perovskite

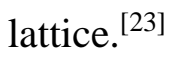

Apart from ionic radii constraints an overall neutral charge must also be achieved. If the A site cation is monovalent and the $\mathrm{X}$ site is occupied by a halogen, then the B site metal must be divalent. However, there are exceptions to this rule, as equal number of +1 and +3 metal cations can yield an overall +2 charge. In this notion the formation of more complex mixed-valent perovskites (e.g., $\left.\mathrm{Cs}_{2} \mathrm{Au}(\mathrm{I}) \mathrm{Au}(\mathrm{III}) \mathrm{I}_{6}\right)$ is also feasible, which further enriches the already broad family of perovskites. ${ }^{[24,25]}$

\subsubsection{Synthetic Strategies to Prepare Perovskites}

A plethora of synthesis strategies are available to prepare HOIP layers with different morphological features. The ultimate goal is to gain control over the overall crystallization process and hence the different physicochemical parameters of the perovskite layer. This enables fine-tuning of the perovskite layer properties towards a specific optoelectronic application. In the next section the most prominent synthesis techniques are briefly introduced (Figure 4). 

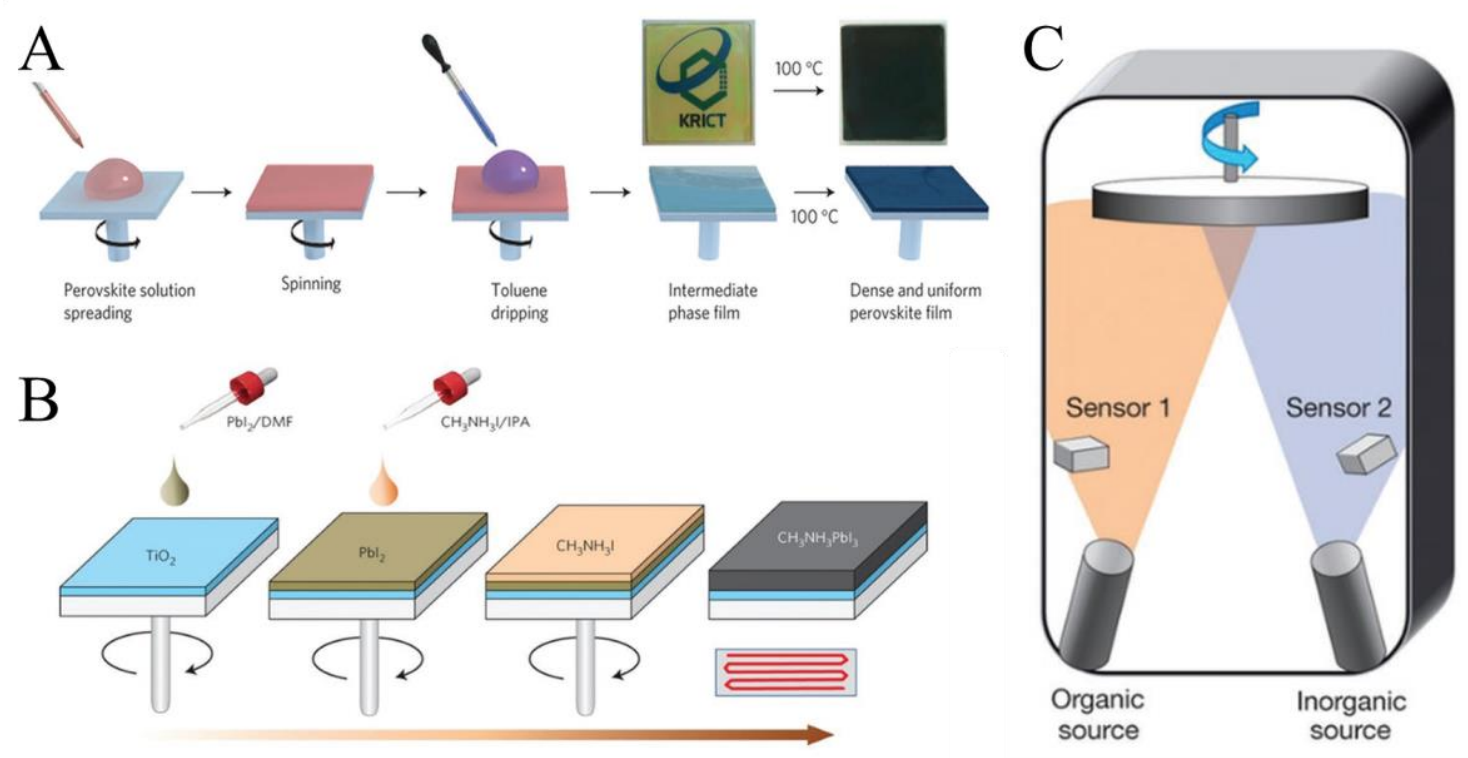

Figure 4. HOIP thin film deposition methods. A: Antisolvent assisted spin-coating method. B: Two-step (sequential) deposition spin-coating method. C: Vapor deposition (coevaporation) method. Adopted from ref. ${ }^{[26]}$

\section{One-step Solution Process - Solvent Engineering}

During one-step methods, the HOIP precursors are dissolved in the same solution and subsequently deposited on the substrate (Figure 4A). Variations in the type of solvent, ${ }^{[27]}$ concentration (ratio) of precursors, ${ }^{[28,29]}$ annealing conditions, ${ }^{[30]}$ and halide composition ${ }^{[28]}$ can change the morphology of the films and thus have an effect on its optoelectronic properties. ${ }^{[31]}$ In the initial studies the most common pitfalls of HOIP layer preparation were inhomogeneous surface coverage ${ }^{[30]}$ and non-uniform film thickness. ${ }^{[32]}$ It was shown that an effective strategy to overcome these issues was the treatment of the wet-precursor film with an antisolvent during spin coating. ${ }^{[33,34]}$ This induces rapid crystallization of the perovskite and results in a film with micron-sized crystals. ${ }^{[35]}$ Apart from the use of antisolvents, additives can also improve the film morphology. For instance, the addition of $\mathrm{Cl}^{-[28,31,36,37]}$ or 2-aminoethanol ${ }^{[38]}$ has an effect on the crystallization mechanism, and also alters the chemical nature of the film forming precursor phases. These additives can even bestow the perovskite films with enhanced water resistance. ${ }^{[38]}$

Usually, high boiling point polar aprotic solvents such as dimethyl sulfoxide (DMSO), N,N-dimethylformamide (DMF), N-methyl-2-pyrrolidone (NMP), and $\gamma$ butyrolactone (GBL) are used for the preparation of the precursor solution. In these solvents the transition metals with an $\mathrm{s}^{2}$ electron configuration that occupy the B-site in HOIPs readily undergo complexation with halide ions. The formed complexes in the solution serve as 
precursors during the film formation (in the case of lead these are called "plumbates"). ${ }^{\text {[3] }}$ The solvents can donate a pair of electrons to form Lewis adducts with $\mathrm{Pb}^{2+}{ }^{[40]}$ Thus it is imperative to understand the solution chemistry of the formed lead complexes, to prepare high-quality perovskite thin films. ${ }^{[29,41,42]}$ Depending on the composition of the solution the solid-state precursor of the film formation can have markedly different features, which ultimately governs the final properties of the perovskite films. ${ }^{[29]}$ In this vein the spectator anions in the solution can also have an effect on the kinetics of the film formation. ${ }^{[43]}$

\section{Sequential (Two-step) Process}

The preparation of HOIP films can also be achieved by various sequential (two-step) methods (Figure 4B). ${ }^{[4]}$ During these processes, a pre-deposited $\mathrm{PbX}_{2}$ film (where $\mathrm{X}$ is usually a halide) is instantaneously converted to the desired perovskite by its exposure to methylammonium halide solution. It has been shown that this method is especially potent in converting $\mathrm{PbI}_{2}$ deposited in a mesostructured substrate. ${ }^{[45]}$ However, when dealing with planar substrates some residual $\mathrm{PbI}_{2}$ remains in the perovskite film.

High quality HOIP films can be formed through an interdiffusion process as well, where the deposition of precursors and the conversion process is decoupled. ${ }^{[46]}$ To completely eliminate the dissolution of the perovskite layer the $\mathrm{PbI}_{2}$ films were exposed to methylammonium-halide vapors (VASP - vapor assisted solution process). This method utilizes the kinetic reactivity of methylammonium iodide (MAI) and the thermodynamic stability of the perovskite during the in situ growth process.

\section{Vacuum Deposition Methods}

Vacuum deposition methods (Figure 4C) favor the formation of extremely uniform, smooth, pinhole free perovskite layers with crystallites in the scale of hundred nanometers. ${ }^{[47]}$ The HOIP thin films are prepared by co-evaporation of the precursors. The main advantage over solution processed films is that the perovskite layer thickness is even and can be precisely controlled. ${ }^{[32]}$ Furthermore, vacuum deposition methods offer the ability to prepare thin films with multiple HOIP layers over large areas, with controllable doping. ${ }^{[48]}$

\section{Single Crystal Growth}

Single crystals are ideal model systems to unravel the fundamental physical properties and ultimate performance limits of HOIP optoelectronic materials. Several methods are available to grow high quality HOIP single crystals. Three methods are frequently used: (i) antisolvent 
vapor assisted, ${ }^{[49]}$ (ii) cooling a saturated solution, ${ }^{[50]}$ and (iii) retrograde solubility approaches. ${ }^{[51-53]}$

An unusual property of many HOIPs is their loss of solubility upon heating, which is termed retrograde solubility. This rare phenomenon is caused by the coordination of solvate molecules and plumbates. The applicable solvent is different for each perovskite: $\mathrm{MAPbCl}_{3}$ - DMSO or DMSO/DMF mixture, ${ }^{[52]} \mathrm{MAPbBr}_{3}-\mathrm{DMF}^{[51]}$ and $\mathrm{MAPbI}_{3}-\mathrm{GBL}^{[51]}$. There are two requirements to observe retrograde solubility in a solvent: the solvent should form stable complexes with the plumbates in solution, and these complexes should dissociate at elevated temperatures.

\subsubsection{Optoelectronic Properties}

Compared to conventional semiconductors, HOIPs possess unique electronic properties, mainly dictated by the $\left[\mathrm{BX}_{6}\right]^{4-}$ octahedra in the structure. According to theoretical calculations in $\mathrm{APbI}_{3}$ type perovskites I $5 \mathrm{p}-\mathrm{Pb} 6 \mathrm{~s}$ forms the valence band (VB), and I $6 \mathrm{p}-$ $\mathrm{Pb} 6 \mathrm{p}$ forms the conduction band (CB). ${ }^{[54]}$ The VB maximum and $\mathrm{CB}$ minimum lies at the same point in reciprocal space, thus halide perovskites are usually direct bandgap semiconductors. As the electronic structure is dominated by the $\left[\mathrm{BX}_{6}\right]^{4-}$ units the A site cation has little or no influence on the band positions. Thus, $\mathrm{CsPbX}_{3}$ and $\mathrm{MAPbX}_{3}$ perovskites have analogous electronic structures. Moreover, the similar dispersion at the band edges results in comparable electron and hole effective masses in HOIPs. Theoretical calculations yielded a $\mathrm{m}_{\mathrm{e}} / \mathrm{m}_{\mathrm{o}}=0.19$ and $\mathrm{m}_{\mathrm{h}} / \mathrm{m}_{\mathrm{o}}=0.25$ value for $\mathrm{MAPbI}_{3},{ }^{[55]}$ which agrees well with the results of high-field magnetoabsorption spectroscopic studies. ${ }^{[56]}$ In the next section various properties that make HOIPs attractive are discussed in detail (Figure 5). 


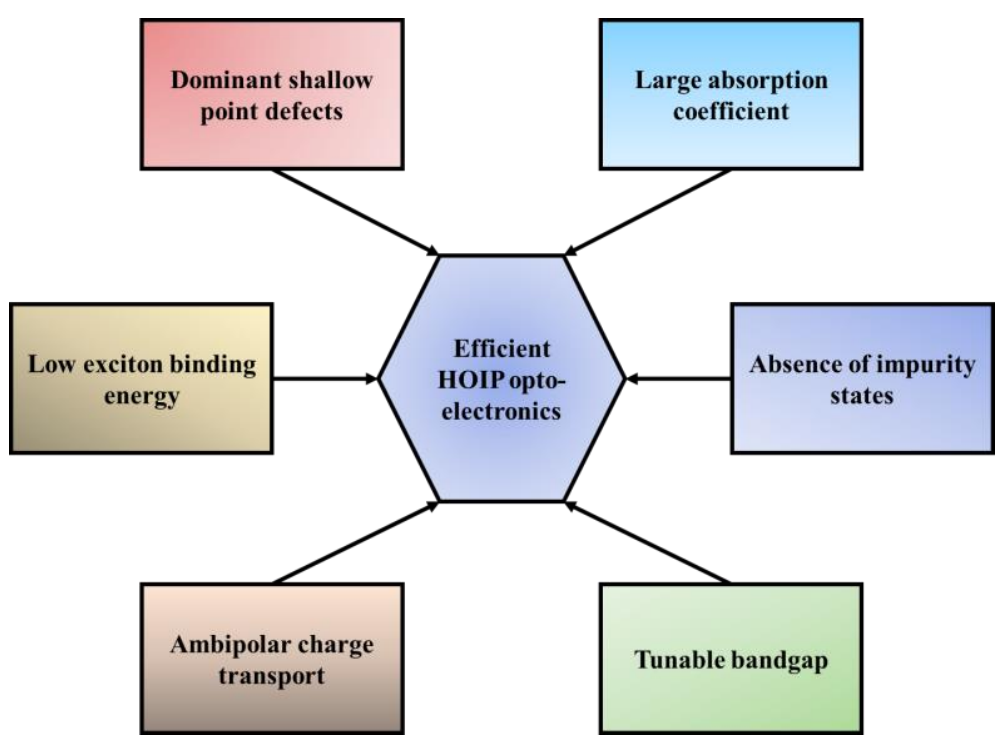

Figure 5. Summary of optoelectronic properties of HOIPs.

\section{Large Absorption Coefficient}

Efficient photovoltaic materials must possess a large absorption coefficient. This is important for two reasons: it enables efficient light harvesting, and is a prerequisite for a large open-circuit voltage $\left(\mathrm{V}_{\mathrm{OC}}\right)$ of a derived device. HOIPs are among the most efficient light absorbers, as such $\mathrm{MAPbI}_{3}$ has an absorption coefficient greater than $3.0 \cdot 10^{4} \mathrm{~cm}^{-1}$ in the visible region. ${ }^{[57,58]}$ The strong absorption property of this materials family is attributed to the coexistence of a direct bandgap and the metal $\mathrm{p} \rightarrow$ halide $\mathrm{p}$ transition. ${ }^{[58]}$ This $\mathrm{p}-\mathrm{p}$ optical transition is stronger than the p-s transition seen in other thin-film solar cell materials. The cause of this is the lone pair effect of the B site metal, thus the CB of these perovskites can be derived from the unoccupied $\mathrm{p}$ orbitals of the metal. These $\mathrm{p}$ orbitals have less dispersion than s orbitals resulting in a greater density of states along the CB edges. ${ }^{[58]}$

Because of the large absorption coefficient, the active layer thickness in PSCs is in the range of $0.3-0.6 \mu \mathrm{m}$, which is three orders of magnitude thinner as in the case of silicon solar cells. Apart from reducing material cost, this also impacts the magnitude of charge carrier recombination in the devices. In thinner configurations, photogenerated carriers do not have to travel far before they are collected, thus they are less prone to recombination. This in turn affects the $\mathrm{V}_{\text {OC }}$ of the derived devices. ${ }^{[57,59]}$ Finally, we note that any distortion in the crystal lattice can cause variation in the optical properties of perovskites. Therefore, it is imperative to preserve the $3 \mathrm{D} \mathrm{B}-\mathrm{X}$ framework to achieve high efficiencies. 


\section{Absence of Impurity States}

The steepness of the optical absorption edge of a material can be characterized by the Urbach energy. Disorder of the microstructure or high density of deep traps can result in the exponential curvature of the absorption near the edge. This sub-bandgap absorption can contribute to $\mathrm{V}_{\mathrm{OC}}$ loss of a device. HOIPs possess small Urbach energy $\left(\mathrm{MAPbI}_{3} \sim 15 \mathrm{meV}\right)$ which further enhances their optoelectronic properties. ${ }^{[60]}$ Furthermore, dramatic improvement in device performance can be achieved through $\mathrm{Cs}^{+}$incorporation, which reduces the Urbach energy and in turn increases the maximum attainable $\mathrm{V}_{\text {OC. }}{ }^{[61,62]}$

\section{Tunable Bandgap}

The bandgap of HOIPs lies in the wavelength regime of visible light $(1.2 \mathrm{eV}-3.0 \mathrm{eV})$. Through simple modifications to the perovskite structure or composition the electronic properties and thus the bandgap of these materials can be fine-tuned.

As theoretical calculations predicted, the A-site cation has negligible contribution to the band structure of the HOIPs. ${ }^{[63]}$ However, the incorporation of different sized cations into the lattice can cause distortions in the perovskite framework, which in turn have an effect on the electronic properties of the material. ${ }^{[64-66]}$ The most prominent feature is that through mixing A-site cations phase stabilization of elusive perovskite phases can be achieved (as seen in the case of $\alpha-\mathrm{FAPbX}_{3}$ ). ${ }^{[61,67,68]}$

The B-site cation is one of the fundamental elements that govern the electronic structure of perovskites. Any exchange on this site leaves the perovskite framework and lattice parameters intact. Through the gradual $\mathrm{Sn}(\mathrm{II})-\mathrm{Pb}(\mathrm{II})$ exchange in $\mathrm{MAPb}_{1-\mathrm{x}} \mathrm{Sn}_{\mathrm{x}} \mathrm{I}_{3}$ continuous variation of the optoelectronic properties was achieved $\left(E_{\mathrm{g}}=1.17-1.55 \mathrm{eV}\right){ }^{[69-}$ ${ }^{71]}$ Interestingly, this exchange shows deviation from Vegard's law. Namely, the bandgap of the solid solutions is not a linear function of composition (lattice parameter). The mixed compositions all showed lower bandgap values compared to the pure compounds. ${ }^{[71]}$

$\mathrm{X}$-site anion exchange has the most prominent effect on the optical properties of HOIPs. In mixed-halide compounds the bandgap monotonously decreases in the series of $\mathrm{Cl}^{-}>\mathrm{Br}^{-}>\mathrm{I}^{-}$. By gradually varying the anion composition of $\mathrm{CsPbCl}_{\mathrm{x}} \mathrm{Br}_{3-\mathrm{x}}$ and $\mathrm{CsPbBr}_{\mathrm{x}} \mathrm{I}_{3-\mathrm{x}}$ nanocrystals their photoluminescence can be precisely tuned between $400-700 \mathrm{~nm} .{ }^{[72,73]} \mathrm{A}$ similar bandgap tunability was found for $\mathrm{MAPbCl}_{\mathrm{x}} \mathrm{Br}_{3-\mathrm{x}}$ and $\mathrm{MAPbBr}_{\mathrm{x}} \mathrm{I}_{3-\mathrm{x}}$ (Figure 6A) as well. ${ }^{[7-77]}$ This substitution mainly affects the VB position of the compounds, as the halide $\mathrm{p}$ orbitals have a large contribution to the VB of perovskites (Figure 6B). In the case of 
$\mathrm{MAPbBr}_{\mathrm{x}} \mathrm{I}_{3-\mathrm{x}}$ this translates to a bandgap variation between $1.6 \mathrm{eV}(\mathrm{x}=0)$ and $2.3 \mathrm{eV}(\mathrm{x}=$ 3). In the case of $\mathrm{Cl} / \mathrm{Br}$ and $\mathrm{Br} / \mathrm{I}$, all intermediate alloys can be realized, however the large ionic size mismatch between $\mathrm{Cl} / \mathrm{I}$ raises a miscibility limit for these alloys (in the case of $\mathrm{MAPbCl}_{\mathrm{x}} \mathrm{I}_{3-\mathrm{x}} \sim 5 \% \mathrm{Cl}^{-}$content). ${ }^{[28,31,36,37]}$ This alloying procedure can be used as a postsynthetic procedure to convert pure perovskite thin films into their mixed-halide counterparts. ${ }^{[74,78]}$ However, such mixed-halide perovskites suffer from photoinduced phase segregation of the solid solutions. ${ }^{[79]}$ During this phenomenon separate domains are formed rich in only one of the constituent halides. ${ }^{[80]}$ This effect is discussed in detail in Section 1.1.5.
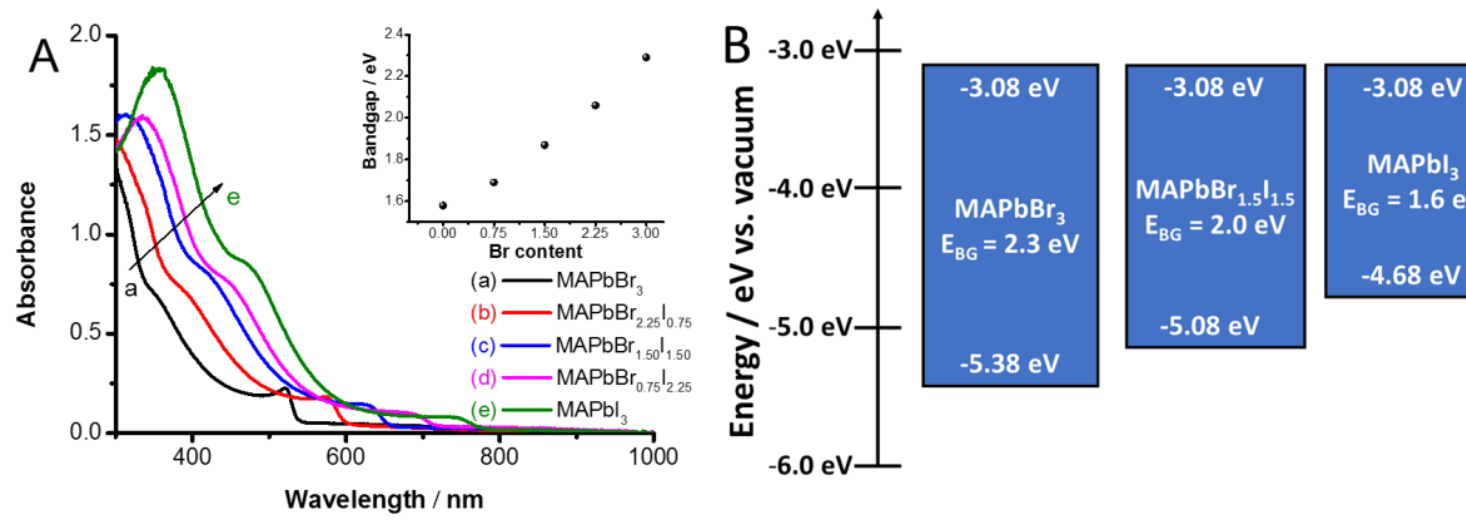

$\mathrm{MAPbl}_{3}$

$E_{B G}=1.6 \mathrm{eV}$

$-4.68 \mathrm{eV}$

Figure 6. A: UV-vis absorption spectra of MAPbBr ${ }_{x} I_{3-x}$ thin layers. The inset shows the determined optical bandgap from the onset of the absorption spectra. B: Representation of the electronic band diagram of $M A P b B r_{x} I_{3-x}$ compounds.

Crystallinity of the samples also influences the measured bandgap. Compared to polycrystalline films $\mathrm{MAPbX}_{3}$ single crystals exhibit narrower bandgaps. ${ }^{[49,50,53,81]}$ This can be attributed to lower trap densities due to higher-dimensional structurally coherent units that are tighter in single crystals compared to their polycrystalline counterparts.

\section{Low Exciton Binding Energy}

In the early solar cell studies HOIPs were used in a similar fashion to organic sensitizers in dye-sensitized solar cells (DSSCs). ${ }^{[2,83]}$ During the evolution of PSC architectures, it was found that the perovskite light absorbers can function effectively in a planar configuration even without employing an electron transport layer $(\mathrm{ETL})^{[32,84]}$ or a hole transport layer $\left(\right.$ HTL ${ }^{[85,86]}$. This behavior raised questions about their photophysical properties, especially about the interplay between excitons and free charge carriers in the material.

Excitons are composed of an electrostatically bound electron-hole pair. Free excitons can be considered as the lowest excited state in an ideal crystalline material that can freely 
move through the lattice, without the transfer of a net charge. The ionization of an exciton to separate charge carriers is thus a crucial aspect in optimizing photovoltaic devices (carrier extraction). For $\mathrm{MAPbI}_{3}$ low binding energy (Mott-Wannier type) excitons are predicted. ${ }^{[19]}$ These can easily dissociate, thus numeric simulations suggest that free charge carriers dominate these materials. ${ }^{[87]}$ It has been shown that the charge carrier generation for both $\mathrm{MAPbI}_{3}$ and $\mathrm{MAPbBr}_{3}$ shows non-excitonic bevavior. ${ }^{[8,89]}$ Furthermore, the external quantum efficiency of $\mathrm{MAPbBr}_{3}$ based devices is unaffected by the use of an external electrical field, which would have an effect if the charge carrier generation in these materials were excitonic in nature. ${ }^{[89]}$

\section{Ambipolar Charge Transport}

The small effective mass of electrons and holes in $\mathrm{MAPbI}_{3}$ signals high charge carrier mobility (inverse relationship). ${ }^{[19,90]}$ Furthermore, the fact that PSCs can function without the use of specific ETL ${ }^{[32,84]}$ or HTL ${ }^{[85,86]}$ also indicates good ambipolar electron and hole transport property of the material. This notion was confirmed by quenching experiments, where selective electron and hole extraction was achieved. The electron-hole diffusion length was in the range of $\sim 100 \mathrm{~nm}$ in solution processed $\mathrm{MAPbBr}_{3}$ and $\mathrm{MAPbI}_{3}{ }^{\left[{ }^{[1,92]}\right.}{ }^{\text {The }}$ investigation of $\mathrm{MAPbI}_{3}$ single crystals revealed a charge carrier diffusion length of $\sim 8-10$ micrometers. ${ }^{[49,51]}$ Currently, the record is held by mm thick $\mathrm{MAPbI}_{3}$ single crystals, where charge carrier diffusion lengths greater than $175 \mu \mathrm{m}$ under 1 sun illumination were shown. ${ }^{\text {[93] }}$ This long-ranged, balanced charge carrier diffusion can be considered a general property of HOIP single crystals as the diffusion length is $\sim 9 \mu \mathrm{m}$ in $\mathrm{MAPbCl}_{3},{ }^{[52]}$ and is $\sim 4-17 \mu \mathrm{m}$ in $\mathrm{MaPbBr}_{3}{ }^{[49,51]}$.

The reason for the long charge carrier diffusion length lies in the rate of different recombination scenarios (Figure 7) in the perovskite layer. ${ }^{[19,90,94,95]}$ Both monomolecular (first-order, i.e., geminate recombination of exciton and trap- or impurity-assisted recombination) and bimolecular (second-order) recombination rates are extremely low in HOIPs. Although Auger recombination (third-order) rates were found to be high, however this recombination pathway is only dominant at high light intensities. ${ }^{[96]}$ 


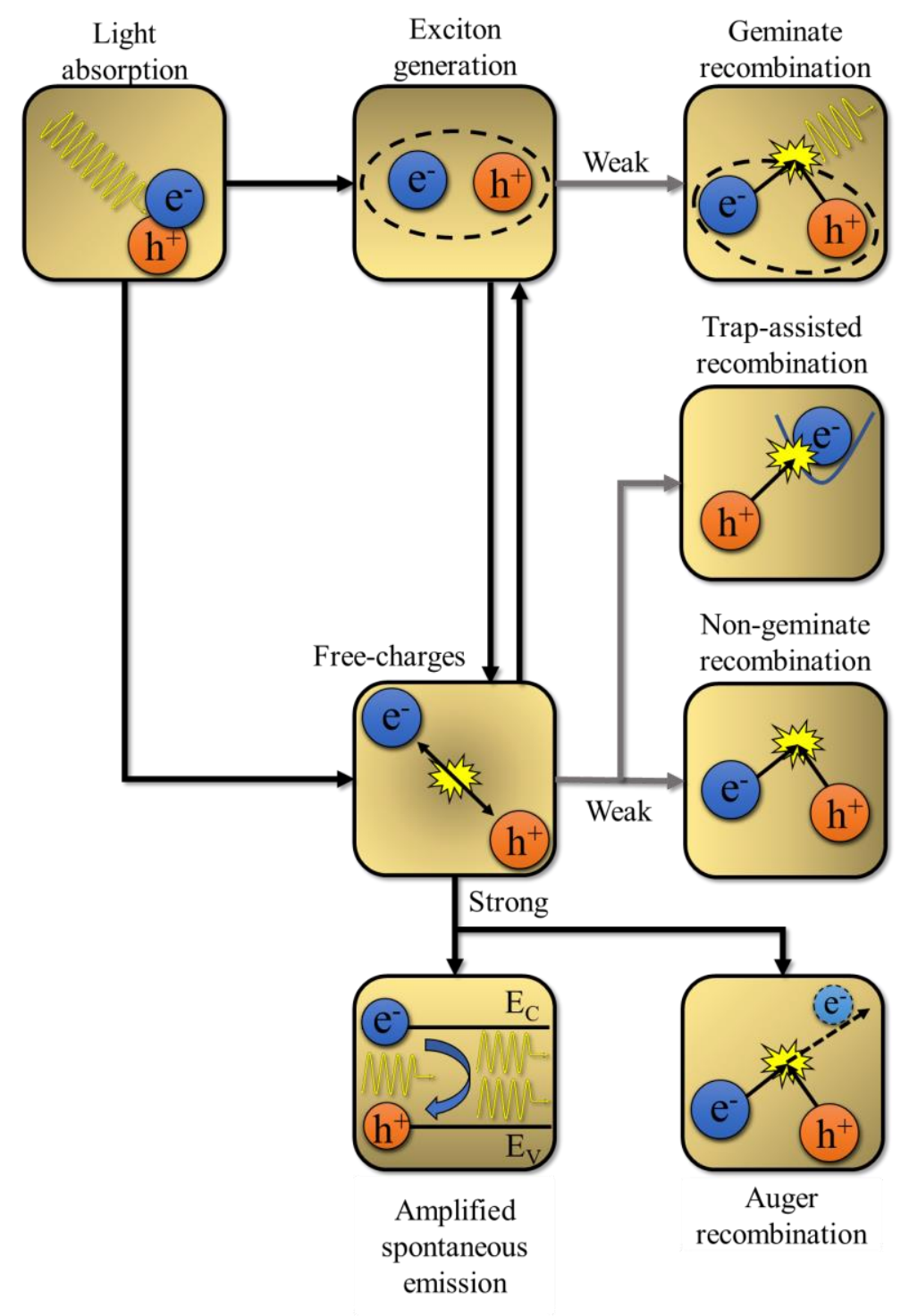

Figure 7. Schematic representation of the various photophysical processes in perovskites following photoexcitation. Adapted from ref. ${ }^{[00]}$.

\section{Dominant Shallow Point Defects}

One of the key features that allows perovskites to deliver remarkable solar cell efficiencies is their unique defect tolerance. ${ }^{[97,98]}$ Defect states in a semiconductor can promote recombination processes, and act as scattering centers for free-charge carriers, thus they deleteriously influence the overall charge collection process. ${ }^{[99]}$ Most HOIPs are fabricated through low-temperature solution processes and still possess small trap densities. The bulk trap density in polycrystalline perovskite thin-films is $\sim 10^{15}-10^{17} \mathrm{~cm}^{-3}$, which becomes even lower $\sim 10^{10} \mathrm{~cm}^{-3}$ in perovskite single crystals. ${ }^{[49,81]}$ In comparison, the trap density of polycrystalline silicon: $\sim 10^{15} \mathrm{~cm}^{-3},{ }^{[100]} \mathrm{GaAs}: \sim 10^{17} \mathrm{~cm}^{-3},{ }^{[101]} \mathrm{TiO}_{2}: \sim 10^{17} \mathrm{~cm}^{-3} \cdot{ }^{[102]}$ This low 
trap density in polycrystalline perovskite thin films can be attributed to their low crystallization activation energy barrier.

Based on the large difference between trap densities of polycrystalline and single crystal perovskites, interfaces and grain boundaries can also act as extended defects. This is mainly caused by the altered stoichiometry of the material on the surface. This holds true for the surface of single crystals as well. ${ }^{[103]}$ Furthermore, the measured defect density is proved to be facet-dependent. ${ }^{[104]}$ To alleviate surface traps, tuning the synthesis conditions to achieve preferred grain orientation or post-synthesis surface passivation strategies are employed. ${ }^{[105]}$ Although theoretical calculations suggest that grain boundaries are inherently benign, and they do not generate gap states, making polycrystalline perovskites single crystal like, ${ }^{[58]}$ the exact nature of grain boundaries in PSCs remains an open question to be answered.

\subsubsection{Instability of Perovskites}

Several different phenomena can induce degradation in HOIP layers. Some of them originate from the environment itself, while others arise from the specific solar cell architectures that are currently used. Environmental factors, even in a completed solar cell architecture (quasisealed), can compromise the integrity of the perovskite absorber layer. Diffusion of chemical species (e.g., $\mathrm{H}_{2} \mathrm{O}$ or $\mathrm{O}_{2}$ ) through the HTL is not hindered and can induce different chemical reactions. In the next section the antagonists of HOIP layers are scrutinized (Figure 8).

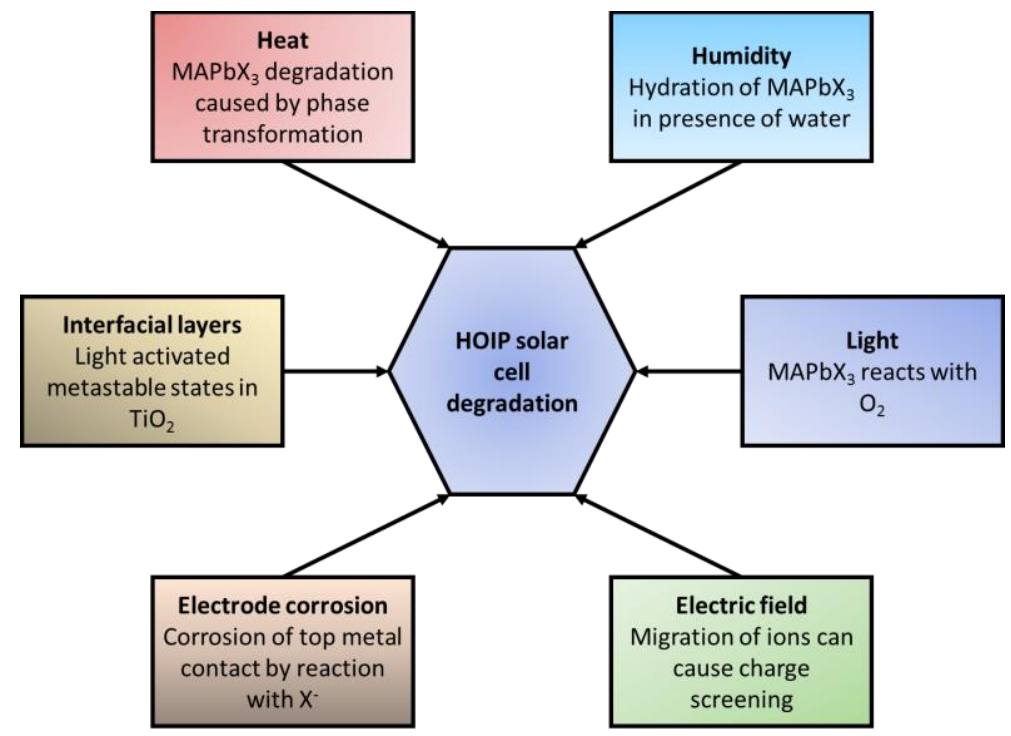

Figure 8. Important factors governing the stability HOIPs. 


\section{Effect of Humidity}

Perhaps the Achilles heel of HOIPs is their sensitivity to polar solvents, especially water. ${ }^{[41,106-110]}$ The overall reaction for the degradation of $\mathrm{MAPbI}_{3}$ is described by $\mathbf{E q .} \mathbf{3}$ as:

$$
\mathrm{CH}_{3} \mathrm{NH}_{3} \mathrm{PbI}_{3}(\mathrm{~s}) \rightarrow \mathrm{CH}_{3} \mathrm{NH}_{2}(\mathrm{~g})+\mathrm{HI}(\mathrm{g})+\mathrm{PbI}_{2}(\mathrm{~s})
$$

However, the actual decomposition reaction is more complex with multiple intermediate hydrated species. ${ }^{[106-108]}$ This alteration affects the light absorption characteristics and induces morphological changes, where the perovskite crystals smoothen and become more oriented. ${ }^{[106,107]}$ The detailed mechanism is described by Eq. 4-5. As the first step, hydrogen bonding between water molecules and the $\mathrm{MA}^{+}$in the lattice disrupts the $3 \mathrm{D}$ perovskite framework. As a result, the structure is gradually transformed into lower dimensional perovskite structures. First 2D perovskite sheets are formed that are separated by water, which leads to the formation of isolated $\left[\mathrm{PbI}_{6}\right]^{4-}$ octahedral units. The first step, where $\mathrm{MAPbI}_{3} \cdot \mathrm{H}_{2} \mathrm{O}$ is formed, is completely reversible. ${ }^{[108]}$

$$
\begin{gathered}
4 \mathrm{CH}_{3} \mathrm{NH}_{3} \mathrm{PbI}_{3}+4 \mathrm{H}_{2} \mathrm{O} \rightleftharpoons 4\left[\mathrm{CH}_{3} \mathrm{NH}_{3} \mathrm{PbI}_{3} \cdot \mathrm{H}_{2} \mathrm{O}\right] \\
4\left[\mathrm{CH}_{3} \mathrm{NH}_{3} \mathrm{PbI}_{3} \cdot \mathrm{H}_{2} \mathrm{O}\right] \rightleftharpoons\left(\mathrm{CH}_{3} \mathrm{NH}_{3}\right)_{4} \mathrm{PbI}_{6} \cdot 2 \mathrm{H}_{2} \mathrm{O}+3 \mathrm{PbI}_{2}+2 \mathrm{H}_{2} \mathrm{O}
\end{gathered}
$$

Under ambient conditions, the formation of alternative degradation products other than $\mathrm{PbI}_{2}$ was also confirmed. During the destruction of the 3D perovskite structure the presence of amorphous $\alpha-\mathrm{PbO}, \mathrm{PbCO}_{3}$, and $\mathrm{Pb}(\mathrm{OH})_{2}$ was also shown. ${ }^{[110]}$ In a similar manner, ammonia vapor can also rapidly degrade the $\mathrm{MAPbI}_{3}$ phase. ${ }^{[11]}$ The nature of the halide in the perovskite structure affects the overall water sensitivity of the compound, which increases in the $\mathrm{Cl}^{-}<\mathrm{Br}^{-}<\mathrm{I}^{-}$order. The mixing of a more moisture resistant halide to a sensitive system can improve the stability of the formed perovskite, however at the expense of device efficiency. ${ }^{[77]}$

\section{Effect of Light}

It has been found that illumination even in the absence of moisture can degrade $\mathrm{MAPbI}_{3}{ }^{[112-}$ ${ }^{114]}$ The proposed mechanism is described by Eq. 6-8. As a first step $\mathrm{MAPbI}_{3}$ is excited and photogenerated electrons and holes are formed. Electrons from the $\mathrm{CB}$ of $\mathrm{MAPbI}_{3}$ can be transferred to adsorbed oxygen on the surface, thus forming superoxide anions. The formed superoxide can simultaneously deprotonate $\mathrm{MA}^{+}$and oxidize $\mathrm{I}^{-}$, thus compromising the stability. Note that the formation of water in Eq. 8 can initiate previously described 
degradation pathways. As expected, however perovskite films stored in nitrogen were stable under steady state illumination. ${ }^{[12-114]}$

$$
\begin{gathered}
\mathrm{CH}_{3} \mathrm{NH}_{3} \mathrm{PbI}_{3} \stackrel{h v>\mathrm{Eg}_{g}}{\longrightarrow} \mathrm{CH}_{3} \mathrm{NH}_{3} \mathrm{PbI}_{3}^{*} \\
\mathrm{O}_{2} \stackrel{\mathrm{CH}_{3} \mathrm{NH}_{3} \mathrm{PbI}_{3}^{*}}{\longrightarrow} \mathrm{O}_{2}^{--} \\
\mathrm{O}_{2}^{--}+\mathrm{CH}_{3} \mathrm{NH}_{3} \mathrm{PbI}_{3} \longrightarrow \mathrm{CH}_{3} \mathrm{NH}_{2}+\mathrm{PbI}_{2}+0.5 I_{2}+\mathrm{H}_{2} \mathrm{O}
\end{gathered}
$$

It was observed that the degradation reaction is more rapid when $\mathrm{Al}_{2} \mathrm{O}_{3}$ is used as the mesoporous scaffold (instead of $\mathrm{TiO}_{2}$ ). ${ }^{[112-114]}$ Thus, the use of $\mathrm{TiO}_{2}$ can suppress this decomposition, by extracting the formed electrons from the $\mathrm{MAPbI}_{3}$ surface before they can react with the adsorbed oxygen. The opposite effect can also be achieved when $\mathrm{MAPbI}_{3}$ layers are forward biased in the dark and in the presence of $\mathrm{O}_{2} \cdot{ }^{[14]}$ Under these circumstances electrons are injected into the $\mathrm{MAPbI}_{3}$ layer which initiate the rapid deterioration of the active layer.

\section{Effect of Heat}

It is crucial to assess the thermal stability of HOIPs layers for two distinct reasons: to optimize the annealing temperature during film deposition, and to ensure device stability during operation. ${ }^{[109,115]}$

During preparation the perovskite films are annealed for a brief amount of time at moderately high temperatures in the $\sim 100{ }^{\circ} \mathrm{C}$ regime. This removes any excess solvent or additive that would remain in the film. Furthermore, it facilitates the formation of the perovskite crystal structure and ensures a proper coverage. In this sense a balance must be found to remove all residual materials, without the degradation of the perovskite material. However, the thermal stability of $\mathrm{MAPbI}_{3}$ seems somewhat controversial. Thermogravimetric studies suggest that $\mathrm{MAPbI}_{3}$ decomposes at $\sim 300{ }^{\circ} \mathrm{C} \cdot{ }^{[50,116-118]} \mathrm{A}$ weight loss is observed between 270 and $340{ }^{\circ} \mathrm{C}$ and is attributed to the removal of organic components from the structure (i.e., $\mathrm{CH}_{3} \mathrm{NH}_{3} \mathrm{I}$ or $\mathrm{CH}_{3} \mathrm{NH}_{2}$ together with $\mathrm{HI}$ ). Furthermore, $\mathrm{MAPbI}_{3}$ undergoes a tetragonal-cubic phase transition at $56{ }^{\circ} \mathrm{C}^{[119]}$ which greatly limits its application in functioning devices. These issues motivated the exchange of $\mathrm{MA}^{+}$to $\mathrm{FA}^{+}$in the perovskite lattice, to enhance its thermal stability. ${ }^{[67,120]}$ 


\section{Interfacial Layers}

The obvious sensitivity issues regarding HOIPs has drawn the attention away from the other constituents of the PSC architecture. The most commonly used ETL $\left(\mathrm{TiO}_{2}\right)$ was found to be a major contributor to the instability of PSCs. ${ }^{[121]}$ UV-light irradiated solar cells suffered from rapid deterioration of their efficiency which was related to a drop in their charge collection efficiency. Ultimately, this phenomenon was explained by the formation of deep trap states within the mesoporous $\mathrm{TiO}_{2}$ framework caused by the UV irradiation. ${ }^{[121]}$ When a UV-cutoff filter or $\mathrm{Al}_{2} \mathrm{O}_{3}$ was used as the scaffold, this problem disappeared. The deterioration of PSC performance is also related to adsorbed molecular $\mathrm{O}_{2}$, connected to oxygen vacancies in the $\mathrm{TiO}_{2}$ framework $\left(\mathrm{Ti}^{3+}\right.$ ions), which leads to the formation of $\mathrm{Ti}^{4+}-$ $\mathrm{O}_{2}{ }^{-}$charge-transfer complexes. Upon UV excitation, the superoxide radical is oxidized by the photogenerated holes, leading to the desorption of molecular oxygen and the formation of a partially occupied deep trap state in $\mathrm{TiO}_{2} .^{[121]}$

Similarly, the HTL also poses a threat to the stability of PSCs. The additives used in the deposition of the most common hole transport material (HTM) 2,2',7,7'-tetrakis( $\mathrm{N}^{\prime} \mathrm{N}^{\prime}$ di-p-methoxyphenylamine)-9,9'-spirobifluorene (spiro-MeOTAD), can also cause the degradation of the solar cell device. It was shown that in the case of DSSCs 4-tertbutylpyridine can evaporate from devices at low temperatures $\left(\sim 85^{\circ} \mathrm{C}\right) .{ }^{[122]}$ Furthermore, Li-bis(trifluoromethane)-sulfonimide (Li-TFSI), the material used to improve the conductivity of the spiro-MeOTAD layer, is a deliquescent material and can draw moisture from the air into the device.

\section{Electrode Corrosion}

In completely assembled PSC architectures, it was found that corrosion of the metal top contact can also negatively influence the stability of PSCs. In cases where Ag was used, discoloration of the top contact was observed after several days. ${ }^{[123]}$ This can be attributed to the migration of iodide ions through the HTL to the Ag contact, where the formation of AgI occurs. This issue also affects other type of top contacts (e.g., $\mathrm{Ca}, \mathrm{Al}, \mathrm{Cr} / \mathrm{Cr}_{2} \mathrm{O}_{3}$ ). ${ }^{[124]}$ Interestingly, $\mathrm{Au}$ as a top contact is also prone to such corrosion events. ${ }^{[124,125]}$

\section{The Effect of Electric Fields}

Perovskites, especially $\mathrm{CsPbBr}_{3}$ and $\mathrm{CsPbI}_{3}$, are well known ionic conductors, as the activation energy for the migration of the halide-ion vacancy is low. ${ }^{[126]}$ In HOIPs there are several candidates for the migrating species. All perovskite forming ions can participate (in 
the case of $\mathrm{MAPbI}_{3}$ e.g., $\mathrm{MA}^{+}, \mathrm{Pb}^{2+}$ and $\mathrm{I}^{-}$) in the overall migration process. Furthermore, additional species from the decomposition scenarios should be also considered $\left(\mathrm{H}^{+}\right) .{ }^{[127]}$ The likelihood of ion migration (i.e., activation energy of migration) depends on several factors: (i) size of the drifting ion, (ii) distance of the vacancy from the ion, (iii) charge of ions and (iv) crystal structure of the material. Theoretical studies agree that the probability of migration follows the $\mathrm{I}^{-}>\mathrm{MA}^{+}>\mathrm{Pb}^{2+}$ trend. In materials with the $\mathrm{ABX}_{3}$ perovskite structure, the vacancy-mediated diffusion process is the most common (Figure 9A). Experimental studies suggest the dominance of electric field induced migration of $\mathrm{MA}^{+}$and $\mathrm{I}^{-}$ions. A redistribution of $\mathrm{MA}^{+}$occurred when a lateral $\mathrm{MAPbI}_{3}$ solar cell was electrically biased. ${ }^{[127-129]}$ After applying a small electrical field, the $\mathrm{MA}^{+}$ions accumulated in the cathode region and were depleted in the anode and central region of the cell. This phenomena led to a switchable photovoltaic effect in such devices. ${ }^{[127-129]}$ The case of $\mathrm{I}^{-}$migration seemed to be a more elusive problem. At elevated temperatures, the movement of $\mathrm{I}^{-}$through interstitial vacancies was shown. ${ }^{[130]} \mathrm{I}^{-}$migration in a $\mathrm{Pb}$-anode/MAPbI $3 / \mathrm{AgI} / \mathrm{Ag}$-cathode arrangement was also the cause of the formation of $\mathrm{PbI}_{2}$ layer near the $\mathrm{Pb}$ anode. ${ }^{[131]}$ It was also demonstrated that at elevated temperatures the use of electrical bias in a lateral $\mathrm{MAPbI}_{3}$ cell can cause the formation of a thin $\mathrm{PbI}_{2}$ layer in the middle of the cell. The electric field driven movement of this $\mathrm{PbI}_{2}$ layer was explained by the massive movement of $\mathrm{I}^{-}$and $\mathrm{MA}^{+}$ ions. ${ }^{[132]}$ There are several ion-migration pathways that can participate in the overall process (summarized in Figure 9B). Not just point defects, but bulk defects, surface and grain boundaries play an important role in ion migration. The grain size of the perovskite films has a pronounced effect on the extent of ion migration. ${ }^{[129,133]}$ It is more difficult to induce photovoltaic switching in PSCs with large grains and this property is directly proportional to the extent of migration in the layer. ${ }^{[129]}$ 
A

Pb-I plane

MA-I plane
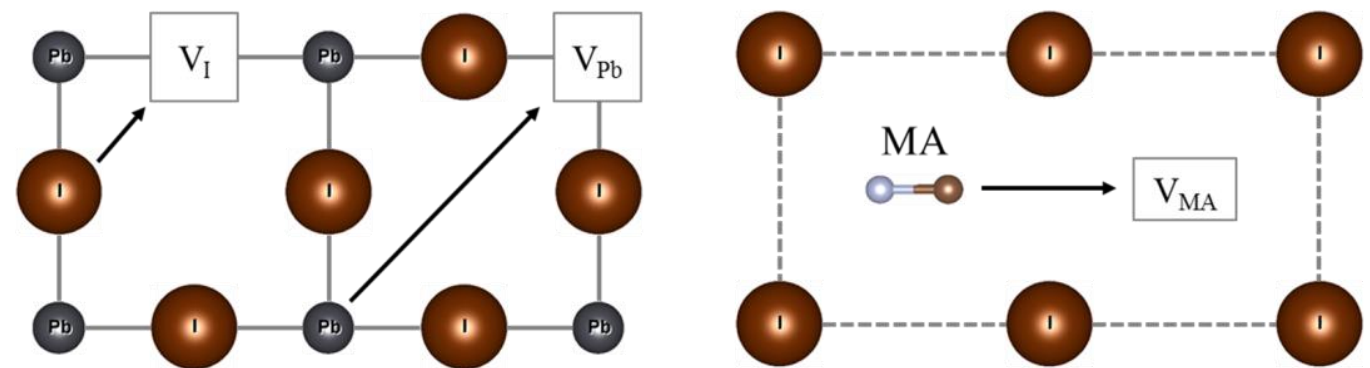

B

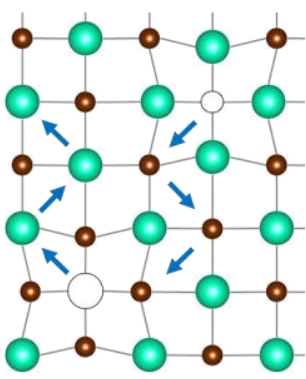

Schottky defects

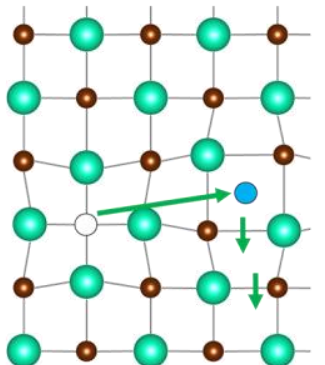

Frenkel defects

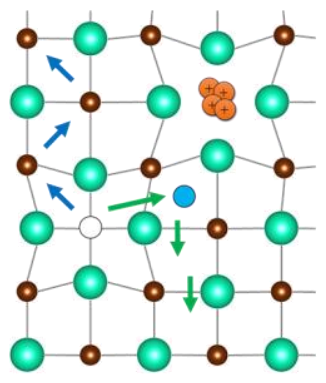

Lattice distortions by accumulated charges/impurities

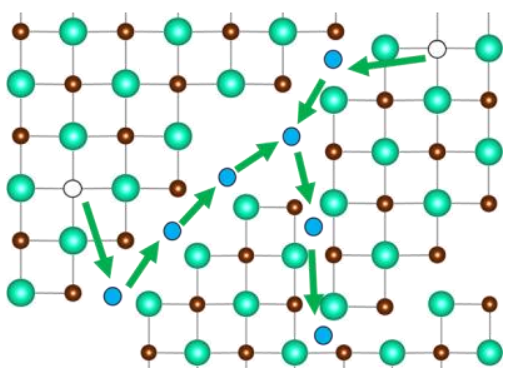

Pathway at grain boundaries

Figure 9. Illustration of the different migration pathways in a $\mathrm{MAPbI}_{3}$ lattice. A: The migration of $\mathrm{I}^{-}, \mathrm{Pb}^{2+}$, and $\mathrm{MA}^{+}$ions through vacancies. B: Different migration pathways considering bulk-point defects, lattice distortions, and grain boundaries. Adapted from ref. [134].

The effect of ion-redistribution on PSC stability and performance is somewhat controversial. It was observed that electric field induced movement of ions can cause the formation of pinholes in the perovskite layer, which can have a detrimental effect on device performance. ${ }^{[129]}$ Furthermore, the formation of $\mathrm{PbI}_{2}$ inside the perovskite absorber layer is also an unwanted phenomenon. ${ }^{[132]}$ Also, by introducing periodic "day/night" cycles into the long term stability test, pronounced fatigue-degradation was observed. This was attributed to the detrimental effect of ion migration during the night cycles. ${ }^{[135]}$ However, it was also shown, that the pronounced ion migration can help to heal an already degraded device. ${ }^{[136,137]}$ It is also interesting, that the migrating $\mathrm{I}^{-}$ions can reach the ETL and react with it. It was shown that employing a [6,6]-phenyl $\mathrm{C}_{61}$ butyric acid methyl ester (PCBM) HTL, the migrating $\mathrm{I}^{-}$effectively doped it, thus improving the performance. ${ }^{[127]}$ In the case of mixedhalide perovskites, the migration of ions have an even more pronounced effect on their optoelectronic properties, as it causes phase-segregation. This phenomenon will be covered 
thoroughly in Section 1.1.5. It is also worth mentioning that electric field induced ionic migration is the main cause of the severe current-voltage hysteresis experienced in PSCs. ${ }^{[138]}$

\subsubsection{Light-Induced Phase Segregation in Mixed Halide Perovskites}

The ability to tune the bandgap of mixed halide perovskites through halide composition is an attractive strategy to design solar cells with tailored response. ${ }^{[61,63,139]}$ Several research groups have evaluated solar cell performance under short-term irradiation and they considered the photovoltaic performance to be stable. ${ }^{[140]}$ Halide ion migration in PSCs plays an important role in determining the overall photovoltaic performance. ${ }^{[141-143]}$ Light soaking, for example, leads to migration of ions and vacancies toward the oxide interface influencing the observed photovoltage. ${ }^{[144]}$ Another property of the mixed halide lead perovskite is its ability to undergo phase segregation under continuous illumination. ${ }^{[0,140,145-148]}$ For example, when $\operatorname{MAPbX}_{3}(\mathrm{X}=\mathrm{Br}, \mathrm{I}, 1: 1$ ratio$)$ is subjected to long-term (> $\left.20 \mathrm{~min}\right)$ visiblelight irradiation, it undergoes phase segregation to yield iodide rich and bromide rich domains as described by Eq. 9.

$$
\begin{gathered}
n M A P b B r_{x} I_{3-x}+h v \rightarrow \\
(n-2 m) M A P b B r_{x} I_{3-x}+m M A P b B r_{x-y} I_{3-x+y}+m M A P b B r_{x+y} I_{3-x-y}
\end{gathered}
$$

Upon stopping the illumination, the segregated phases are restored to the original mixed phase. ${ }^{[147-149]}$ In previous studies, the kinetics of halide ion segregation as well as dark recovery was monitored. The origin of the segregation has been explained using different models. These include entropy of mixing, ${ }^{[145]}$ halide ion defect driven movement of ions, ${ }^{[150]}$ and polaron induced lattice strain. ${ }^{[149]}$ It was also found that the excitation intensity and duration of irradiation determines the dark recovery. ${ }^{[147,150]}$ However, up to this point the effect of halide ion segregation on the performance of PSCs was missing. This raises the obvious question: What is the impact of long-term light irradiation (or light soaking) on the performance of mixed halide perovskite solar cells?

\subsubsection{Electrochemical Properties of Perovskites}

In unraveling the reasons behind the unparalleled optoelectronic properties of HOIPs the use of (photo)electrochemical techniques started to gain a foothold recently. These powerful tools are capable of probing charge carrier generation and transport in HOIPs. Fundamental optoelectronic properties (e.g., band edge positions, distribution of trap states) can be probed 
using spectroelectrochemistry. Furthermore, the use of other coupled techniques can contribute to the understanding the chemical changes at the interfaces and in the electrode material itself upon charge carrier generation. The use of electrochemical techniques is not restricted to probing physicochemical properties of HOIPs but can also be used as a synthetic tool in the assembly of complex perovskite architectures. Electrochemistry can even be invoked as a possible lead removal strategy in HOIP recycling scenarios. ${ }^{[151]}$

The extreme sensitivity of HOIPs to polar solvents made all electrochemical measurements rather challenging. Recently, it was demonstrated that (photo)electrochemical measurements can be performed in dichloromethane-based electrolytes in the presence of different reversible redox couples (e.g., ferrocene and benzoquinone). ${ }^{[152]}$ The assembled liquid junction cells showed remarkable stability for prolonged irradiation. In this notion, electrochemical impedance analysis was performed to estimate the flatband potential and charge carrier density of different HOIPs. ${ }^{[152,153]}$ A very thorough study investigated the electron injection process into formamidinium lead halide perovskite using spectroelectrochemistry. ${ }^{[154]}$ It was demonstrated in this study that it is very difficult, yet possible, to probe band edge positions via charge carrier injection. During these experiments, however, an irreversible electrochemical reduction occurred, thus making the picture even murkier. An overview of the possible solution chemistry events was given, although without taking into account the likely contribution of the electrolyte ions. ${ }^{[154]}$ A similar approach was followed for mixed $\mathrm{CsPbX}_{3}\left(\mathrm{X}=\mathrm{Cl}^{-}, \mathrm{Br}^{-}\right.$, and $\left.\mathrm{I}^{-}\right)$perovskites, where it was assumed that band edge positions can be simply determined from voltammetric data. ${ }^{[155]}$

Apart from determining various physicochemical properties, coupled (photo)electrochemical techniques can offer a fast and convenient way to perform combinatorial analysis of the performance of various HOIP compositions. Scanning electrochemical microscopy can be effectively used to determine the optimal composition of different perovskite compounds in a semiconductor array arrangement. ${ }^{[156,157]}$ In terms of delivering the highest photocurrent, the optimal excess $\mathrm{PbI}_{2}$ content of $\mathrm{MAPbI}_{3}$ layers ${ }^{[56]}$ and the optimal $\mathrm{MASnI}_{3-\mathrm{x}} \mathrm{Br}_{\mathrm{x}}$ composition ${ }^{[157]}$ were determined in a liquid junction setup.

By encapsulating the HOIP layer in a photoelectrode assembly, their use as efficient photocathodes in $\mathrm{H}_{2}$ generation in aqueous media was also shown. ${ }^{[158]}$ The photocathodes retained more than $80 \%$ of their efficiency for $\sim 1 \mathrm{~h}$. The effective protection of the HOIP electrode can pave the way of using these materials in solar fuel generation scenarios. ${ }^{[159]}$ 


\section{Li-ion Batteries}

Halide perovskites of the $\mathrm{CsPbX}_{3}$ family can be considered as fast ionic conductors, because of their high ionic conductivity. ${ }^{[126]}$ This means that their crystal structure remains rather frozen, while ionic species are migrating through the lattice. The complex defect chemistry makes it difficult to discern the diffusivity of specific ions through the perovskite lattice. However, an extrinsic $\mathrm{Li}^{+}$source (e.g., through a solution/perovskite interface) can be used to determine ionic diffusion kinetics in HOIPs. ${ }^{[160]}$ In such a case, electronic and ionic transport is decoupled, because the migration and intercalation of $\mathrm{Li}^{+}$(the slower process) determines the kinetics of the diffusion process. In highly lithiated $3 \mathrm{D} \mathrm{MAPbBr}_{3}$ electrodes the ionic conductivity was found to be $10^{-3} \Omega^{-1} \mathrm{~cm}^{-1}$, which falls in the range of superionic conductors $\left(10^{-4} \Omega^{-1} \mathrm{~cm}^{-1}-10^{-1} \Omega^{-1} \mathrm{~cm}^{-1}\right)$. As the perovskite lithiation progresses, specific capacity values as high as $400 \mathrm{~mA} \mathrm{~h} \mathrm{~g}^{-1}$ were reached. ${ }^{[160,161]} 3 \mathrm{D} \mathrm{MaPbBr} 3$ electrodes exhibit a highly reversible lithium uptake and release without severe distortion of the perovskite lattice (topotactic intercalation). It was proposed that the lithiation proceeds in a fashion that multiple $\mathrm{Li}^{+}$are intercalated into the same unit cell. In this sense, the formation of $\mathrm{Li}_{\mathrm{x}} \mathrm{MAPbBr} 3$ compounds were assumed, where $\mathrm{x}$ approaches $3 . \mathrm{MAPbI}_{3}$ has inferior specific capacitance and stability compared to $\mathrm{MAPbBr}_{3}$ based electrodes. A summary of specific capacitance values can be seen in Table 1. All specific capacitance values for the first discharge cycle greatly surpass the theoretical limit for $\operatorname{MAPbBr}_{3}\left(56 \mathrm{~mA} \mathrm{~h} \mathrm{~g} \mathrm{~g}^{-1}\right)$, which raises suspicion that a conversion event or another effect is also in play. ${ }^{[162]}$ Furthermore, these results are often not supported by any intercalation mechanism. Theoretical calculations and experimental results suggest that in the case of $\mathrm{MAPbCl}_{3}$ and $\mathrm{MAPbBr}_{3}$ the conversion process to $\mathrm{LiX}$ and $\mathrm{Pb}$ metal dominates the electrochemical behavior. ${ }^{[162]}$ 
Table 1. Summary of the performance of the HOIPs used in Li-intercalation studies. In brackets the charging current density is shown.

\begin{tabular}{|c|c|c|c|c|c|}
\hline Perovskite & Used electrolyte & $\begin{array}{l}\text { Potential } \\
\text { window }\end{array}$ & $\begin{array}{c}\text { Specific } \\
\text { capacitance }\end{array}$ & Stability & Reference \\
\hline $\mathrm{MAPbBr}_{3}$ & \multirow{4}{*}{$\begin{array}{l}\text { ethylene carbonate : } \\
\text { ethyl-methyl } \\
\text { carbonate : dimethyl } \\
\text { carbonate }(1: 1: 1) \text { and } \\
1.0 \mathrm{M} \mathrm{LiPF}_{6}\end{array}$} & \multirow{4}{*}{$\begin{array}{c}0.0 \mathrm{~V}-2.0 \mathrm{~V} \\
\text { vs. } \mathrm{Li} / \mathrm{Li}^{+}\end{array}$} & $\begin{array}{l}400 \mathrm{~mA} \mathrm{~h} \mathrm{~g}{ }^{-1} \\
\left(200 \mathrm{~mA} \mathrm{~g}^{-1}\right)\end{array}$ & n.a. & {$[160]$} \\
\hline $\mathrm{MAPbBr}_{3}$ & & & $\begin{array}{l}520 \mathrm{~mA} \mathrm{~h} \mathrm{~g}^{-1} \\
\left(200 \mathrm{~mA} \mathrm{~g}^{-1}\right)\end{array}$ & $\begin{array}{c}30 \% \text { after } 20 \\
\text { cycles }\end{array}$ & [164] \\
\hline $\mathrm{MAPbBr}_{3}$ & & & $\begin{array}{l}330 \mathrm{~mA} \mathrm{~h} \mathrm{~g}^{-1} \\
\left(200 \mathrm{~mA} \mathrm{~g}^{-1}\right)\end{array}$ & $\begin{array}{c}40 \% \text { after } 100 \\
\text { cycles }\end{array}$ & \multirow{2}{*}[161]{} \\
\hline $\mathrm{MAPbI}_{3}$ & & & $\begin{array}{l}44 \mathrm{~mA} \mathrm{~h} \mathrm{~g}{ }^{-1} \\
\left(200 \mathrm{~mA} \mathrm{~g}^{-1}\right)\end{array}$ & $\begin{array}{c}20 \% \text { after } 100 \\
\text { cycles }\end{array}$ & \\
\hline $\mathrm{MAPbI}_{3}$ & \multirow{3}{*}{$\begin{array}{c}\text { ethylene carbonate : } \\
\text { dimethyl carbonate } \\
(1: 1) \\
\text { with } 5 \% \\
\text { fluoroethylene } \\
\text { carbonate and } 1.0 \mathrm{M} \\
\text { LiPF }_{6}\end{array}$} & \multirow{3}{*}{$\begin{array}{c}0.0 \mathrm{~V}-2.5 \mathrm{~V} \\
\text { vs. } \mathrm{Li} / \mathrm{Li}^{+}\end{array}$} & $\begin{array}{l}476 \mathrm{~mA} \mathrm{~h} \mathrm{~g}^{-1} \\
\left(100 \mathrm{~mA} \mathrm{~g}^{-1}\right)\end{array}$ & $\begin{array}{c}40 \% \text { after } 50 \\
\text { cycles }\end{array}$ & \multirow{3}{*}[163]{} \\
\hline$\left(\mathrm{C}_{4} \mathrm{H}_{9} \mathrm{NH}_{3}\right)_{2} \mathrm{PbI}_{4}$ & & & $\begin{array}{l}1605 \mathrm{~mA} \mathrm{~h} \mathrm{~g}^{-1} \\
\left(100 \mathrm{~mA} \mathrm{~g}^{-1}\right)\end{array}$ & $\begin{array}{c}13 \% \text { after } 50 \\
\text { cycles }\end{array}$ & \\
\hline $\mathrm{C}_{6} \mathrm{H}_{9} \mathrm{OPbI}_{3}$ & & & $\begin{array}{l}580 \mathrm{~mA} \mathrm{~h} \mathrm{~g}{ }^{-1} \\
\left(100 \mathrm{~mA} \mathrm{~g}^{-1}\right)\end{array}$ & $\begin{array}{c}40 \% \text { after } 50 \\
\text { cycles }\end{array}$ & \\
\hline
\end{tabular}

The performance of lower dimensional perovskite materials was also evaluated, where, $1 \mathrm{D}$ and 2D HOIPs vastly outperformed their 3D counterparts. ${ }^{[163]}$ A common feature of all $\mathrm{Li}^{+}$intercalation experiments is the strong fading of the specific capacitance over the course of cyclization. In the case of lower dimensional HOIPs, this can be attributed to the irreversible nature of the reduction of $\mathrm{Pb}$ (II) to metallic $\mathrm{Pb}{ }^{[163]}$ Lower dimensional HOIPs are more prone to this effect, which ultimately results in the formation of $\mathrm{Li}_{\mathrm{x}} \mathrm{Pb}_{\mathrm{y}}$ alloys. In the case of 3D HOIPs, no metallic lead formation was found (or the characterization/sensitivity was insufficient) and the fading of the specific capacitance is still unexplained. ${ }^{[164]}$ To exclude the effect of electrolyte on the stability of HOIPs, a solvent toolkit was proposed to perform lithium intercalation experiments in the future. ${ }^{[165]}$

In the case of $\mathrm{CsPbBr}_{3}$ nanocrystals, electrochemical Li-doping was explored. ${ }^{[166,167]}$ Extremely high negative voltages were used to perform $\mathrm{Li}^{+}$intercalation, thus the formation of metallic $\mathrm{Pb}$ was observed. Interestingly, a reversible electrochromic behavior was shown for the $\mathrm{Li}$-doped $\mathrm{CsPbBr}_{3}$ electrodes. However, these studies also lack an exact mechanism behind the observed phenomena or any quantification of the incorporated amount of lithium. 


\section{Electrochemiluminescence}

Electrochemiluminescence (ECL) could be employed to investigate charge carrier transfer under various electrochemical conditions. Most studies on this matter focus exclusively on understanding the properties of $\mathrm{CsPbBr}_{3}$. Several irreversible redox processes were identified by cyclic voltammetry for $\mathrm{CsPbBr}_{3}$ nanocrystal (NC) based electrodes. ${ }^{[168,169]}$ All of these were attributed to the presence of charged states of the NCs. However, this assumption is quite problematic, because it completely disregards the possibility of chemical change (Faradaic processes) of the $\mathrm{CsPbBr}_{3} \mathrm{NCs}$. Electrochemically generated electrons and holes were injected in rapid succession into the $\mathrm{CsPbr}_{3} \mathrm{NC}$ electrodes and the annihilation of the ECL signal was monitored. This was performed by potential switching experiments, where the potential of the $\mathrm{CsPbBr}_{3} \mathrm{NC}$ electrodes was switched rapidly between an electron injecting potential to a hole injecting potential. Relatively weak and fading ECL signals were observed, which were attributed to the unstable nature of the radicals from $\mathrm{CsPbBr}_{3} .{ }^{[168]}$ However, this phenomenon more likely signals the chemical change of the perovskite layer. An increased stability was observed when ethyl-acetate was used as the solvent. It was proposed that ethyl-acetate can act as a co-reactant in the ECL reaction. ${ }^{[169]}$

ECL of both $\mathrm{MAPbBr}_{3}{ }^{[170]}$ and $\mathrm{CsPbBr}_{3}{ }^{[171]}$ were studied in aqueous media as well, but the stability of the films was not addressed. In a recent study, long carbon chain acids or amids were used to stabilize $\mathrm{CsPBBr}_{3} \mathrm{NCs}$ in aqueous media. ${ }^{[172]}$ These ligands orient themselves with their polar head groups, making the surface of the perovskite NCs surface hydrophobic. This strategy was found to stabilize the $\mathrm{CsPbBr}_{3} \mathrm{NCs}$ in aqueous media for a few hours, but the degradation of the layers was still inevitable.

Despite the above examples, solid and coherent knowledge about the fundamental electrochemical behavior of these materials is still missing. In this notion several questions remain unanswered: What is the electrochemical window to safely conduct electrochemical experiments and what are the redox events that were left unexplained?

\subsection{Perovskite Solar Cells}

Long-term device instability is the most pressing issue that hampers PSC commercialization. As more effort was put into understanding the fundamental properties of HOIPs, better control over device stability was achieved. It is imperative that apart from the degradation of HOIPs, the role of the interfaces in the devices must be precisely evaluated. Each interface and contact layer in the device plays a crucial role in determining the overall stability. When 
control over the interfaces is achieved, the devices will be absent from the initial rapid decay (often termed burn-in) and the gradual slower decay of their efficiency. ${ }^{[173]}$ Few studies already reported extremely durable PSCs with over 1000 hours of continuous operation without substantial decay, under ambient conditions (combined stresses of light (including UV light), oxygen and moisture). ${ }^{[173-175]}$

\subsubsection{General Solar Cell Architectures}

To understand how different optoelectronic properties of HOIPs shaped the applicable solar cell architectures a quick overview on the evolution PSCs is presented in the following section (Figure 10). ${ }^{[176]}$

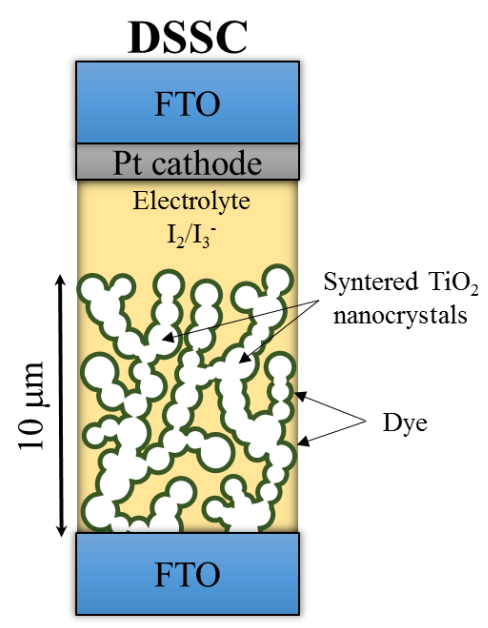

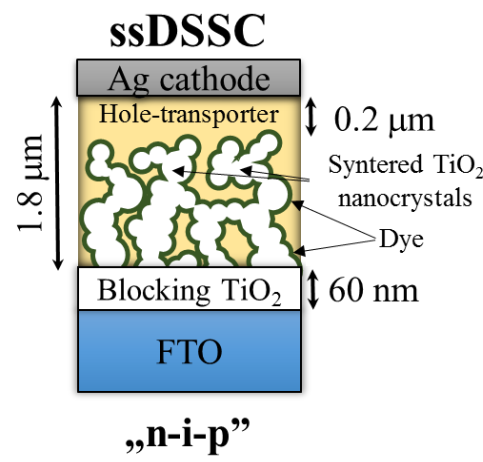

thin-film

perovskite

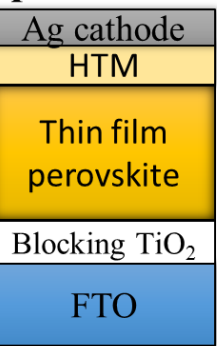

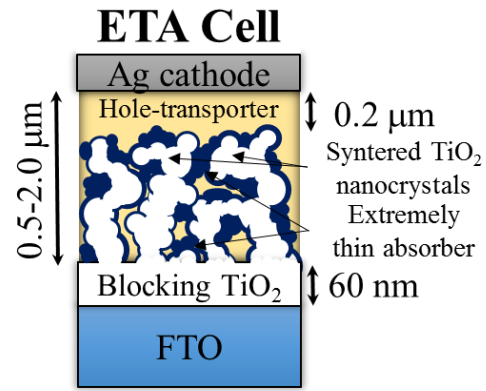

,p-i-n"

thin-film

perovskite

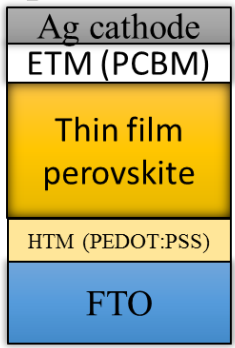

Figure 10. The evolution of PSC architectures. Adapted from ref. ${ }^{[176]}$.

Three components are necessary to fabricate DSSCs: (i) a mesoporous n-type semiconductor scaffold (e.g., $\mathrm{TiO}_{2}$ ), (ii) a light absorber (or sensitizer) anchored on the surface of the scaffold (e.g. organic dyes, inorganic quantum dots), and (iii) a redox active electrolyte (e.g., $\left.\mathrm{I}_{2} / \mathrm{I}_{3}{ }^{-}\right) \cdot{ }^{[177]}$ In these architectures it is imperative to use a high surface area mesoporous scaffold. This ensures that sufficient amount of sensitizer can be adsorbed on 
its surface to maximize the absorption of the incident sunlight. Usually in the case of organic sensitizers this requires films as thick as $10 \mu \mathrm{m}$. HOIPs were initially utilized as alternative sensitizers in DSSC architectures. The pioneering study reported a $3.8 \%$ efficiency for a $\mathrm{MAPbI}_{3}$ based liquid junction cell. ${ }^{[82]}$ This was further improved to $6.5 \%$ by optimizing the mesoporous $\mathrm{TiO}_{2}$ layer properties and the $\mathrm{MAPbI}_{3}$ deposition procedure. ${ }^{\left[{ }^{[3]}\right.}$ Both of these studies pointed out the instability of the HOIP layer to prolonged exposure to the electrolyte. ${ }^{[22,83]}$ To overcome this issue, the use of all-solid state architectures offered a convenient way. This architecture already gained a foothold in the DSSC community because of the fear from leakage of the liquid electrolyte. ${ }^{[178]}$ In these cases, the electrolyte is exchanged to either a small molecule (e.g., spiro-MeOTAD) or a polymeric HTL. ${ }^{[179]}$ However, in such architectures the poor infiltration of the HTL limits the thickness to a few micrometers, thus strongly absorbing materials must be used to obtain high efficiencies. As HOIPs have an order of magnitude larger absorption coefficient then organic dyes, they performed remarkably well in solar cells utilizing extremely thin absorbers. The devices delivered power conversion efficiencies of $7.6 \%$ for $\mathrm{MAPbI}_{2} \mathrm{Cl},{ }^{[180]}$ and $9.7 \%$ for $\mathrm{MAPbI}_{3} \cdot{ }^{[181]}$ Interestingly, when the scaffold was exchanged to insulating mesoporous $\mathrm{Al}_{2} \mathrm{O}_{3}$ the PSCs delivered an increased efficiency of $10.9 \% .{ }^{[180]}$ This is only possible if the perovskite coating is continuous on the surface of the scaffold and can sustain long range electron transport to the back-contact. Furthermore, electron transport through the HOIP is much faster than through the ETL. This arrangement constitutes an entirely new class of solar cell architectures, termed meso-superstructured solar cell. ${ }^{[84,121,180,182]}$ It has been demonstrated that PSCs can function without either an $\operatorname{ETL}^{[32,84]}$ or a HTL ${ }^{[85,86]}$. A twocomponent $\mathrm{TiO}_{2} / \mathrm{MAPbI}_{3}$ solar cell can deliver $5 \%$ efficiency under simulated full sunlight, employing a Au cathode. ${ }^{[183]}$ Even a $300 \mathrm{~nm}$ thick perovskite absorber can generate and transport both electrons and holes to the collecting contacts with close to unity efficiency. ${ }^{\left[{ }^{[4]}\right.}$ As an ultimate demonstration of ambipolar transport, planar heterojunction PSCs showed high efficiencies of over $15 \%{ }^{[32]}$ This finally demonstrated that the perovskite semiconductors can exhibit sufficiently good ambipolar charge transport and participate in all of the principal roles of SC operation: light absorption, charge generation, and transport of both electrons and holes. To realize flexible and low-cost PSCs, an inverted design is generally adopted, as metal-oxide ETL lack the required flexibility for such applications. In an inverted design a HTL (poly(3,4-ethylenedioxythiophene):polystyrene sulfonate (PEDOT:PSS)) is first deposited on the fluorine doped tin oxide (FTO) covered glass. ${ }^{[184]}$ 
The highest efficiency is $19.9 \%$ that was obtained in inverted PSC, which is on par with the best performing regular architecture PSCs. ${ }^{[185]}$

\section{Electron Transport Layer}

PSCs can function efficiently without the use of an ETL, however its presence is necessary to achieve high-performance. Its role is to facilitate electron collection and transport from the perovskite to the current collector electrode. Thus, the material used as an ETL must meet strict requirements. These include: (i) good electron mobility, to enable fast electron transport within the material, (ii) wide bandgap, to ensure high transparency and allow more light to pass through the material, and (iii) compatible energy levels with the employed perovskite, to facilitate charge transfer and efficiently block holes. Currently, mostly inorganic materials are used (e.g., $\mathrm{TiO}_{2}, \mathrm{ZnO}, \mathrm{SnO}_{2}, \mathrm{WO}_{3}$ ), but several organic materials have emerged over the years. ${ }^{[186,187]}$ In a general solid-state DSSC design, apart from the mesoporous- $\mathrm{TiO}_{2}$ layer (mp- $\left.\mathrm{TiO}_{2}\right)$, a blocking- $\mathrm{TiO}_{2}$ layer $\left(\mathrm{bl}-\mathrm{TiO}_{2}\right)$ must be used. It is crucial to use such a compact blocking ETL layer, to prevent charge recombination on the FTO/perovskite interface.

Based on the dimensions of the used $\mathrm{TiO}_{2}$ four categories can be distinguished: (i) OD (nanoparticles) (ii) 1D (nanorods, wires, tubes) (iii) 2D (nanosheets) (iv) 3D (branched nanowires, rods, fibers). The most commonly used building blocks for the mesoporous layer are $\mathrm{TiO}_{2}$ nanoparticles. The size, shape and crystallinity of the nanoparticles ultimately determine the performance of the derived PSCs. Furthermore, the thickness of the layer should also be optimized. The bottleneck in achieving high efficiencies is the infiltration of the perovskite into the labyrinth-like structure. This seems to be a universal challenge, as all the structures suffer from inadequate perovskite filling. ${ }^{[186,187]}$

In the case of flexible electronics, the exchange of the inorganic ETL layer to a more bendable material is necessary. Organic semiconductors are ideal candidates, however the use of in an inverted architecture is necessary in this case. This is caused by the difficulty of preparing a pinhole-free film in a conventional design. The most prominent organic materials are fullerenes such as $\mathrm{C}_{60}$ and PCBM. These materials were found to decrease the density of defects at the perovskite/ETL interface. ${ }^{[186,187]}$

Based on the above considerations, it seems that the electron extracting interface is the most sensitive part of PSCs, thus it is imperative to understand the exact nature of the ETL/perovskite interaction. Furthermore, there are several phenomena (e.g., hysteresis) that 
are tied to this specific interface. Thus, it is important to find an answer for the question: How does the nature of the ETL/perovskite interface influence the charge recombination process?

\subsubsection{Hole Transport Layer}

On the other side of the PSC the HTL serves several purposes: (i) it is a physical/energetic barrier between anode and perovskite layer, efficiently blocking electron transfer to anode, (ii) it improves the efficiency of hole transfer, (iii) influences the VOC of the device, (iv) protects the metal contact from degradation, and (v) might also increase the stability of the PSCs. ${ }^{[188]}$ To deliver high efficiencies, a material should offer: (i) high hole mobility to reduce losses during hole transport, (ii) compatible ionization potential with the HOIP, to minimize injection losses, (iii) high thermal stability and resistance to external degradation factors (e.g., moisture, oxygen and light), and (iv) low cost. HTMs employed in PSCs can be divided into inorganic (e.g., $\mathrm{NiO}, \mathrm{CuSCN}$ and $\mathrm{CuI}$ ) and organic categories. The organic HTMs form three subcategories: (i) small molecules such as spiro-MeOTAD, (ii) oligomers and (iii) polymers (e.g., poly(3-hexylthiophene) (P3HT), PEDOT, and poly[bis(4phenyl)(2,4,6-trimethylphenyl)amine] (PTAA)) (Figure 11). ${ }^{[188,189]}$

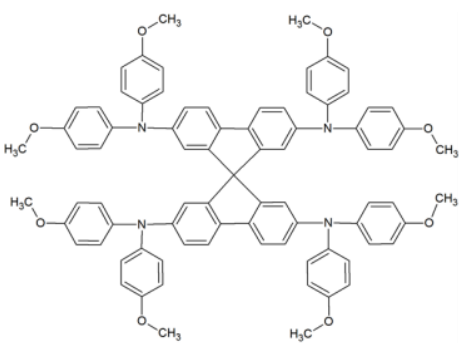

$$
\begin{gathered}
2,2^{\prime}, 7,7^{\prime} \text {-tetrakis(N,N'-di-p- } \\
\text { methoxyphenylamine)-9, } 9 \text { '- } \\
\text { spirobifluorene }
\end{gathered}
$$
Spiro-MeOTAD
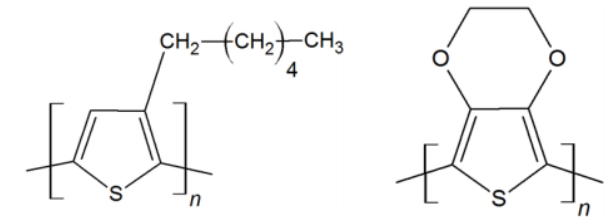

Poly(3-hexylthiophene) Poly(3,4-ethylenedioxythiophene) P3HT

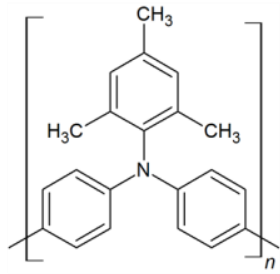

Poly[bis(4-phenyl) $(2,4,6$ trimethylphenyl)amine]

PEDOT

PTAA

\section{Figure 11. Skeletal structural formula of commonly employed organic hole transporter materials.}

Among small molecule HTMs the most widely employed molecule is spiroMeOTAD. Currently it is considered as an essential building block of record breaking PSCs. However, the use of spiro-MeOTAD has drawbacks of its own, such as: (i) high-cost, owing to the multi-step synthesis and difficult purification of the HTM, (ii) the necessity of dopants, caused by the low conductivity of pristine spiro-MeOTAD, and (iii) the sensitivity to atmospheric conditions, which is especially severe, when it is doped with Li-TFSI. The low 
charge carrier mobility in pristine spiro-MeOTAD arises from its inherent triangular pyramid configuration that leads to large intermolecular distances. Dopants such as Li-TFSI and 4tert-butylpyridine are added to suppress charge recombination as well as to improve the contact between absorber and HTM. ${ }^{[188]}$

In pursuit of cost-effective and stable HTMs, polymers with higher hole mobility than dopant-free small molecules, are also considered in PSCs. Few common examples are P3HT, PTAA and PEDOT:PSS which achieved promising power conversion efficiencies in PSCs. ${ }^{[188-190]}$

\subsection{Conducting Polymers as Hole Transporters in Perovskite Solar Cells}

Several research groups focus on the synthesis of new organic HTMs for perovskite PSCs. ${ }^{[188-190]}$ Interestingly, this growing interest has not been accompanied by an improvement of the arsenal of preparation techniques, and spin-coating is almost exclusively employed to deposit these polymer films.

In a $n-i-p$ PSC design, polymeric materials that are soluble in perovskite antisolvents are studied (e.g., P3HT ${ }^{[117,191,192]}$ and PTAA ${ }^{[117]}$ ). Although it was demonstrated that PEDOT accepts holes as effectively as spiro-MeOTAD ${ }^{[193]}$ its use (apart from a few examples ${ }^{[194-}$ ${ }^{196]}$ ) has been restricted to inverted solar cell designs ( $p-i-n$ junction). This limit is rooted in the fact that the direct spin-coating of the PEDOT:PSS aqueous solutions would immediately destroy the perovskite layer. The inverted designs, however, pose further challenges, such as overcoming the energy mismatch between FTO/PEDOT and attaining adequate surface coverage of the perovskite layer (determined by the morphology of the underlying PEDOT film).

Although electrodeposition of the HTM has showed promising results in the case of DSSCs,${ }^{[197]}$ it remained an elusive task for the $n-i-p$ PSC architecture up to this point. This is mainly caused by the instability of $\mathrm{MAPbI}_{3}$ in polar solvents, ${ }^{[198]}$ where most electrochemical syntheses are carried out. Further complications arise from the dynamic exchange between the ions in the perovskite layer and ions present in the solution, which can alter the composition of the perovskite layer. ${ }^{[199]}$ It has been demonstrated recently that the electrochemical properties of $\mathrm{MAPbI}_{3}{ }^{[152,156]}$ and related materials ${ }^{[153-155,157]}$ can be studied in dichloromethane based electrolytes. However, special care must be exercised, because the use of an external electrochemical bias can also induce unintended side reactions (e.g., corrosion of the perovskite layer). Under carefully controlled conditions, 
electrodeposition offers superior control over several efficiency-determining factors (e.g., morphology, regularity, conductivity, optical absorption, and layer thickness). ${ }^{[197]}$ This raises the question: Is it possible to employ electrochemical deposition methods and use conducting polymers as the HTL of HOIP based solar cells in a n-i-p design? 


\section{Aims}

During my research I aimed to find answers to the questions raised in the introduction:

- What is the impact of long-term light irradiation (or light soaking) on the performance of mixed halide perovskite solar cells?

- What is the electrochemical window to safely conduct electrochemical experiments and what are the redox events that were left unexplained?

- How does the nature of the ETL/perovskite interface influence the charge recombination process?

- Is it possible to employ electrochemical deposition methods and use conducting polymers as the HTL of HOIP based solar cells in a n-i-p design?

As the first step, I aimed to find a solvent/electrolyte system, where electrochemical measurements could be carried out. For these experiments, the chosen model systems were $\mathrm{FTO} / \mathrm{TiO}_{2} / \mathrm{CsPbBr}_{3}$ and $\mathrm{FTO} / \mathrm{TiO}_{2} / \mathrm{MAPbI}_{3}$. My aim was to identify the redox alterations of these materials and to ultimately determine an electrochemical stability window. As a next step, I coupled transient absorption spectroscopy with electrochemical methods to elucidate the role of $\mathrm{TiO}_{2}$ in the charge recombination process. As electrochemistry can be also used as a synthetic tool, I also investigated the possibility to electrochemically prepare a PEDOT $\mathrm{HTL}$ in $\mathrm{MAPbI}_{3}$ electrodes, thus forming a regular $n-i-p$ junction solar cell. As an introduction to solar cell characterization and PSC preparation I also studied the interaction of light with mixed-halide PSCs. I investigated the effect of phase segregation on the optical and electronic properties of mixed halide solar cells. 


\section{Materials and Methods}

\subsection{Materials}

Chemicals used for the synthesis of $\mathrm{CsPbBr}$ nanocrystals

- lead (II) bromide ( $\mathrm{PbBr}_{2}$ - Alfa Aesar, $\left.99.999 \%\right)$

- cesium carbonate $\left(\mathrm{Cs}_{2} \mathrm{CO}_{3}\right.$, Alfa Aesar, $\left.99.9 \%\right)$

- oleic acid (OAc, Sigma Aldrich, $90 \%$ technical grade)

- oleylamine (OAm - Sigma Aldrich, $70 \%$ technical grade)

- 1-octadecene (ODE, Sigma-Aldrich, $95 \%$ )

- acetone (Fischer Scientific, HPLC grade)

- n-hexane (Sigma-Aldrich, $95 \%$ anhydrous)

- n-heptane (Sigma-Aldrich, $99 \%$ spectrophotometric grade)

- ethanol (KOPTEC, 200 proof, anhydrous)

Chemicals used for the preparation of the $M A P b B r_{x} I_{3-x}$ solutions

- lead (II) iodide ( $\mathrm{PbI}_{2}$ - Sigma-Aldrich, Ultradry, beads, - 10 mesh, $\left.99.999 \%\right)$

- lead (II) bromide $\left(\mathrm{PbBr}_{2}-\right.$ Alfa Aesar, 98+\%)

- methylammonium-iodide (MAI - Dyesol)

- methylammonium bromide (MABr - Dyesol)

- dimethylsulfoxide (DMSO - Sigma-Aldrich, anhydrous, $\geq 99.9 \%$ )

- n,n-dimethyl formamide (DMF - Sigma-Aldrich, anhydrous, $99.8 \%$ )

Chemicals used for the preparation of bis-EDOT

- 3,4-ethylenedioxythiophene (EDOT - Sigma Aldrich, $97 \%$ )

- tetrahydrofurane (THF - VWR, $99.7 \%$ )

- n-buthyl-lithium (n-BuLi - Alfa Aesar 2.5 M in hexane)

- copper(II) chloride $\left(\mathrm{CuCl}_{2}\right.$ - Sigma Aldrich, $\left.99 \%\right)$

Chemicals used for the preparation of the bl-TiO 2 and $m p-\mathrm{TiO}_{2}$ layer

- titanium diisopropoxide bis(acetylacetonate) (Sigma-Aldrich, $75 \mathrm{wt} \%$ in isopropanol)

- 1-butanol (Sigma-Aldrich, $99.8 \%$ ) 
- $\quad$ titanium (IV) chloride ( $\mathrm{TiCl}_{4}-\mathrm{Alfa}$ Aesar, $\left.99.6 \%\right)$

Chemicals used for the electrochemistry experiments

- 3,4-ethylenedioxythiophene (EDOT - Sigma Aldrich, $97 \%$ )

- dichloromethane (DCM - Sigma Aldrich, anhydrous, $\geq 99.8 \%$, contains 40-150 ppm amylene as stabilizer, dried over $3 \AA$ molecular sieves)

- tetrabutylammonium-hexafluorophosphate $\left(\mathrm{Bu}_{4} \mathrm{NPF}_{6}-\right.$ Sigma Aldrich, for electrochemical analysis, $\geq 99.0 \%$ )

- tetrabutylammonium-tetrafluoroborate $\left(\mathrm{Bu}_{4} \mathrm{NBF}_{4}-\right.$ Sigma Aldrich, for electrochemical analysis, $\geq 99.0 \%$ )

- tetrabutylammonium-perchlorate $\left(\mathrm{Bu}_{4} \mathrm{NClO}_{4}-\right.$ Sigma Aldrich, for electrochemical analysis, $\geq 99.0 \%$ )

\subsection{Synthesis}

\subsubsection{Synthesis of $\mathrm{CsPbBr}_{3}$ Nanocrystals}

The Cs-oleate precursor (0.306 $\mathrm{g} \mathrm{Cs}_{2} \mathrm{CO}_{3}, 2.8 \mathrm{~cm}^{3}$ of OAc and $3 \mathrm{~cm}^{3} \mathrm{ODE}$ ) was injected into a $\mathrm{PbBr}_{2}$ precursor solution $\left(0.808 \mathrm{~g} \mathrm{PbBr}_{2}, 6 \mathrm{~cm}^{3} \mathrm{OAc}, 6 \mathrm{~cm}^{3} \mathrm{ODE}\right.$ and $\left.6 \mathrm{~cm}^{3} \mathrm{OAm}\right)$ at $170{ }^{\circ} \mathrm{C}$. Highly luminescent, yellow colored $\mathrm{CsPbBr}_{3} \mathrm{NCs}$ were formed immediately after injection. The NCs were cooled to room temperature, purified via centrifugation, carefully washed with acetone, and re-suspended in $3 \mathrm{~cm}^{3}$ of a 95/5 V/V\% n-hexane/n-heptane mixture before further use.

\subsubsection{Synthesis of $\mathrm{CH}_{3} \mathrm{NH}_{3} \mathrm{PbBr}_{x} \mathrm{I}_{3-x}$}

A one-step synthesis was used for the preparation of $\mathrm{MAPbBr}_{\mathrm{x}} \mathrm{I}_{3-\mathrm{x}}$ solutions, which is based on the previously described Lewis base adduct method. ${ }^{[40]}$ The MAPbBr $_{\mathrm{x}} \mathrm{I}_{3-\mathrm{x}}$ precursors are mixed together followed by the addition of DMSO so that the molar ratio of $\mathrm{MAPbBr}_{\mathrm{x}} \mathrm{I}_{3-\mathrm{x}}$ : DMSO is 1:1. To prepare thin films for the spectroelectrochemistry experiments, a precursor concentration of $0.36 \mathrm{M}$ was used, while this was increased to $1.5 \mathrm{M}$ for the preparation of solar cells. Prior to spin-coating the solutions were stirred for $1 \mathrm{~h}$ at room temperature and were filtered with an inorganic membrane filter $(0.2 \mu \mathrm{m}$ pore size, G8549141, Whatman) before use. 


\subsubsection{Synthesis of Bis-EDOT}

The preparation of bis-EDOT was carried out by Ullmann coupling, using lithiated EDOT and copper(II)-chloride. ${ }^{[200]}$

\subsection{Methods}

\subsubsection{Electrode/Solar Cell Preparation}

The presented work relies on three different architectures (Figure 12). The PSCs consisted of a FTO electrode coated with a thin bl- $\mathrm{TiO}_{2}$ layer, a mp- $\mathrm{TiO}_{2}$ layer, the perovskite absorber layer, a spiro-MeOTAD layer and a gold contact completed the solar cell (Figure 12A). To study light-induced segregation phenomena solar cell architectures without the top gold contact were also prepared (Figure 12B). To mimic the solar cell architectures, the electrodes for the electrochemistry studies were prepared almost identically. The preparation procedure was the same until the perovskite deposition. A thinner layer of perovskite was used, to ensure significant transmittance of the layers in a wide wavelength range. In the case of electrodes, the upper HTL and gold contacts were omitted (Figure 12C).
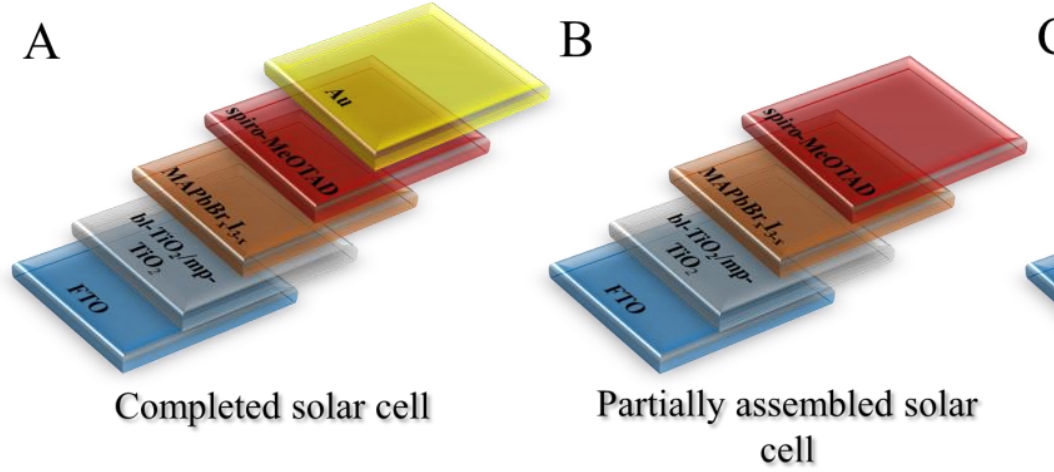

C

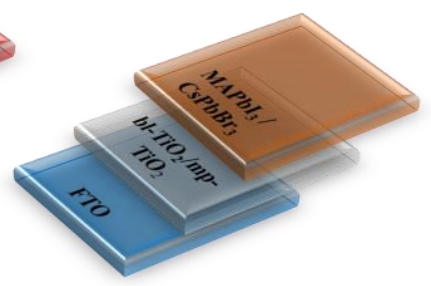

Electrode

Figure 12. Schematic representation of the different architectures used throughout the experiments. A: Complete solar cell, B: partially complete solar cell and $\boldsymbol{C}$ : electrode.

Briefly, the FTO substrates were washed with soap, rinsed with deionized water, sonicated in ethanol for 20 minutes and finally plasma cleaned prior to use. First a $\mathrm{TiO}_{2}$ blocking layer was spin-coated from a $0.15 \mathrm{M}$ titanium diisopropoxide bis(acetylacetonate) solution in 1-butanol. Immediately after spin-coating, the layer was subjected to a heat treatment for 5 minutes at $125{ }^{\circ} \mathrm{C}$ under glovebox conditions $\left(\mathrm{N}_{2}\right.$ atmosphere, $\mathrm{H}_{2} \mathrm{O}<0.1$ ppm, $\left.\mathrm{O}_{2}<0.1 \mathrm{ppm}\right)$. This was followed by the spin-coating of a mesoporous $\mathrm{TiO}_{2}$ layer from an ethanol-based suspension with a concentration of $0.12 \mathrm{~g} \mathrm{~cm}^{-3}$. Details about the $\mathrm{TiO}_{2}$ paste used for the preparation of the suspension can be found elsewhere. ${ }^{[201]}$ After spin- 
coating, the mesoporous layers were calcined for 1 hour at $550{ }^{\circ} \mathrm{C}$ in air. The FTO/bl$\mathrm{TiO}_{2} / \mathrm{mp}-\mathrm{TiO}_{2}$ samples were then subjected to a $\mathrm{TiCl}_{4}$ treatment step. For this, aqueous solution of $20 \mathrm{mM} \mathrm{TiCl}_{4}$ was prepared and the samples were immersed into it at $90{ }^{\circ} \mathrm{C}$ for 10 minutes. This step was followed by a final calcination step for 30 minutes at $500{ }^{\circ} \mathrm{C}$ in air.

The $\mathrm{FTO} / \mathrm{TiO}_{2} / \mathrm{CsPbBr}_{3}$ electrodes were prepared with a method using subsequent cycles of NC deposition and annealing. Deposition of the NCs on the substrate was followed by annealing at $250{ }^{\circ} \mathrm{C}$ for 3 minutes, and this cycle was repeated until bulk films were formed with the desired optical density of $\mathrm{A}_{518 n m} \sim 0.8$. The $\mathrm{CsPbBr} 3$ coated electrodes were kept in glovebox $\left(\mathrm{N}_{2}\right.$ atmosphere, $\left.\mathrm{H}_{2} \mathrm{O}<0.1 \mathrm{ppm}, \mathrm{O}_{2}<0.1 \mathrm{ppm}\right)$ after preparation and used immediately.

The FTO/TiO $/ \mathrm{MAPbI}_{3}$ electrodes were prepared by an antisolvent assisted one step method. During spin-coating, the samples were subjected to an antisolvent treatment step with diethyl-ether (Sigma-Aldrich, for HPLC, $\geq 99.9 \%$, inhibitor-free). After the spincoating, the samples were immediately transferred to a preheated hot-plate $\left(65^{\circ} \mathrm{C}\right.$ for $\left.1 \mathrm{~min}\right)$ to ensure evaporation of the antisolvent. This was followed by an annealing step at $100{ }^{\circ} \mathrm{C}$ for $2 \mathrm{~min}$.

\subsubsection{Spin-coating Protocols}

bl- $\mathrm{TiO}_{2}$ - a three-step dynamic coating procedure on a $5 \mathrm{~cm}$ x $5 \mathrm{~cm}$ substrate: (1) at $700 \mathrm{rpm}$ for $8 \mathrm{~s}$, (2) at $1000 \mathrm{rpm}$ for $10 \mathrm{~s}$ and (3) $2000 \mathrm{rpm}$ for $40 \mathrm{~s}$ (acceleration $1000 \mathrm{rpm}$ ). $0.5 \mathrm{~cm}^{-3}$ of $\mathrm{bl}^{-\mathrm{TiO}_{2}}$ solution was used.

mp- $\mathrm{TiO}_{2}$ - a one-step static coating procedure on a $5 \mathrm{~cm}$ x $5 \mathrm{~cm}$ substrate: (1) at 2000 rpm for $20 \mathrm{~s}$ (acceleration $1000 \mathrm{rpm}) .1 .0 \mathrm{~cm}^{-3}$ of mp- $\mathrm{TiO}_{2}$ suspension was used.

$\mathrm{MAPbBr}_{\mathrm{x}} \mathrm{I}_{3-\mathrm{x}}-\mathrm{a}$ one-step static coating procedure on a $2.5 \mathrm{~cm}$ x $2.5 \mathrm{~cm}$ substrate: (1) at $4000 \mathrm{rpm}$ for $25 \mathrm{~s}$ (acceleration $1200 \mathrm{rpm}$ ). $50 \mu 1$ of perovskite solution was used. 0.5 $\mathrm{cm}^{-3}$ diethyl ether was dispensed on the spinning substrate in a continuous motion in less than $4 \mathrm{~s}$ during the early stage (6 s elapsed time) of the film formation.

$\mathrm{CsPbBr}_{3}$ - dynamic coating procedure on a $2.5 \mathrm{~cm}$ x $2.5 \mathrm{~cm}$ substrate: at $5000 \mathrm{rpm}$ for $20 \mathrm{~s}$ (acceleration $1000 \mathrm{rpm}$ ) $20 \mu \mathrm{l}$ of stock solution, and annealing at $250{ }^{\circ} \mathrm{C}$ for $3 \mathrm{~min}$. 


\subsubsection{Preparation of $\mathrm{CH}_{3} \mathrm{NH}_{3} \mathrm{PbI}_{3}$ Solar Cells}

During the preparation of PSCs multiple layers are spin-coated on an FTO substrate. schematic representation of the different layers was already shown in Figure 12A. As the preparation of PSCs requires multiple steps, it is important to know whether there are no errors during the fabrication procedure. To mitigate the effect of human error during solar cell preparation $\mathrm{MAPbI}_{3}$ solar cells were fabricated regularly (Figure 13A). The summary of the efficiencies of these cells are shown in Figure 13B. Several flaws of the preparation procedure were weeded out during the initial period and the use of only the highest-grade chemicals was justified. To be able to draw meaningful conclusions it was imperative to reproducibly fabricate these solar cells.
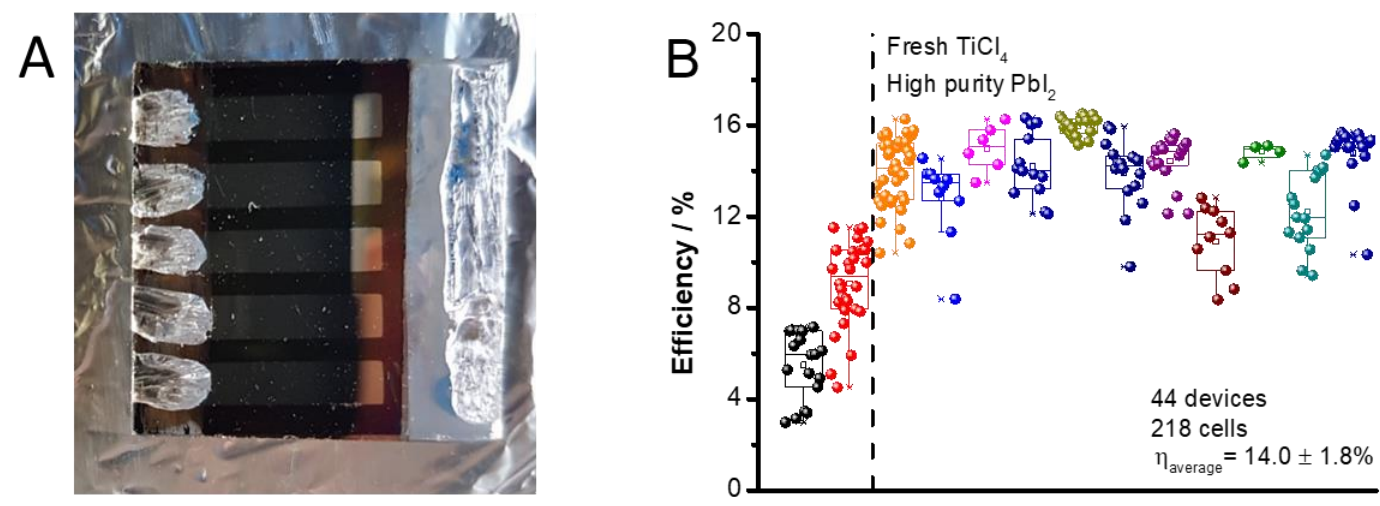

Figure 13. A: Photograph of a fully assembled MAPbI 3 solar cell. B: The evolution of $\mathrm{MAPbI}_{3}$ solar cell efficiencies throughout the course of the work.

\subsubsection{Inducing Phase Segregation}

To induce phase segregation in the solar cells, they were subjected to white light irradiation with an incident power density of $100 \mathrm{~mW} \mathrm{~cm}^{-2}$. The light from the Xe-lamp source was passed through an AM 1.5 filter beforehand. To ensure that thermal effects have miniscule contribution to the phase segregation process, a water filter was placed in front of the samples.

\subsubsection{Electrodeposition of PEDOT}

The electrodeposition of PEDOT hole transporter on the $\mathrm{FTO} / \mathrm{TiO}_{2} / \mathrm{CH}_{3} \mathrm{NH}_{3} \mathrm{PbI}_{3}$ electrodes was carried out from a $0.01 \mathrm{M}$ bis-EDOT and $0.1 \mathrm{M} \mathrm{Bu}_{4} \mathrm{NPF}_{6}$ containing dichloromethane electrolyte. The deposition potential and the growth of the PEDOT layers were monitored 
by potentiodynamic methods coupled with spectroelectrochemistry. Ultimately, however potentiostatic deposition was used to control the thickness of the deposited PEDOT layers, by controlling the polymerization charge density. To remove the contribution of the charging/discharging of the electrochemical double layer to the passed charge density a pretreatment step was introduced prior to the electrodeposition at $\mathrm{E}=+0.5 \mathrm{~V}$ for $60 \mathrm{~s}$ in the polymerization media. After the polymerization was finished the solar cells were rinsed with dichloromethane (as-is solar cells). In the case of post-treated solar cells, the post treatment step was carried out immediately after the polymerization.

\subsection{Physical Characterization}

\subsubsection{Electrochemistry/Spectroelectrochemistry}

The electrochemical measurements were carried out with a Gamry potentiostat in a standard three-electrode setup (see the schematics of the setup in Figure 14). The $\mathrm{FTO} / \mathrm{TiO}_{2} /$ perovskite electrodes functioned as the working electrode, a $\mathrm{Pt}$ mesh $\left(1 \mathrm{~cm}^{2}\right)$ as the counter electrode (cleaned by "Piranha solution", water and finally DCM), and a $\mathrm{Ag} / \mathrm{AgCl}$ wire as a pseudo-reference electrode. The home-made $\mathrm{Ag} / \mathrm{AgCl}$ pseudo-reference electrode was a $\mathrm{Ag}$-wire having $\mathrm{AgCl}$ deposited on its surface. This is generally applied in organic media, because it can be considered as a secondary electrode, with very low chloride ion concentration. Its potential was calibrated before and after the experiments, by measuring the formal potential of the ferrocene/ferrocenium redox couple in DCM (0.01 M ferrocene and $0.1 \mathrm{M} \mathrm{Bu}_{4} \mathrm{NPF}_{6}$ ). Cyclic voltammetry was used, and the formal potential was found to be $\mathrm{E}=+0.45 \pm 0.04 \mathrm{~V}$ vs. our $\mathrm{Ag} / \mathrm{AgCl}$. All cells were assembled in a glovebox $\left(\mathrm{N}_{2}\right.$ atmosphere, $\mathrm{H}_{2} \mathrm{O}<0.1 \mathrm{ppm}, \mathrm{O}_{2}<0.1 \mathrm{ppm}$ ) and sealed hermetically to ensure inert conditions.

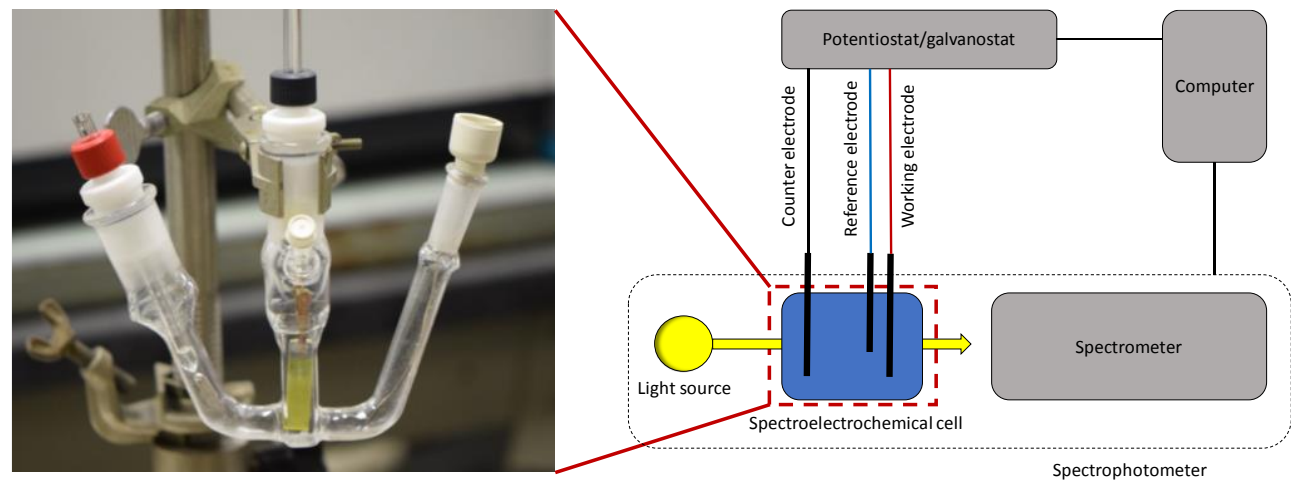

Figure 14. Schematic representation of the spectroelectrochemical setup, together with the photograph of the cell. 
Prior to electrochemical experiments all conducting salts were dried in a vacuum oven under the conditions listed in Table 2. The water content of the solutions was monitored by Karl Fischer titration, and kept below 10 ppm.

Table 2. Drying conditions of the different conducting electrolytes.

\begin{tabular}{|c|c|c|c|}
\hline Conducting salt & Melting point $/{ }^{\circ} \mathrm{C}$ & $\begin{array}{c}\text { Drying } \\
\text { temperature } /{ }^{\circ} \mathrm{C}\end{array}$ & Time / hours \\
\hline $\mathrm{Bu}_{4} \mathrm{NBF}_{4}$ & 160 & 140 & 6 \\
\hline $\mathrm{Bu}_{4} \mathrm{NClO}_{4}$ & 220 & 180 & \\
\hline $\mathrm{Bu}_{4} \mathrm{NPF}_{6}$ & 240 & 180 \\
\hline
\end{tabular}

\section{Cyclic Voltammetry}

During cyclic voltammetry $(\mathrm{CV})$, the potential of the working electrode is changed between two vertex potentials with a constant sweep rate, while the current response of the system is measured (potentiodynamic technique). When a sufficient potential value is reached to drive a certain chemical reaction (e.g., corrosion, electropolymerization) the system responds with a current increase (in absolute value), depending on the rate of the reaction (this is in addition to the non-characteristic capacitive current recorded for the given electrode). From a cyclic voltammogram the onset potential then can be determined, which corresponds to least amount of energy to drive the reaction at a detectable rate at a given electrode under specific circumstances (such as temperature, ionic strength of solution or type of the electrolyte).

\section{Potentiostatic Measurements}

During potentiostatic measurements a constant potential is applied (with respect to the reference electrode) to the working electrode, while the current response of the system is measured. By integrating the current-time curve the passed charge can be determined.

\section{In situ Spectroelectrochemistry}

If an electrochemically induced redox event (either in the solution or on the electrode surface) is coupled with a color change, UV-vis spectroscopy (Varian Cary 50 Bio) can be used to monitor the corresponding color change. Correlating the current response with the absorbance change of the electrode can help distinguish between distinct electrode reactions (e.g. corrosion, electropolymerization). This coupled technique can help evaluate the 
reversibility of certain reactions. Most of the electrochemical techniques can be coupled with $\mathrm{UV}$-vis spectroscopy as long as a transparent electrode and electrochemical cell is used.

\subsubsection{Ultrafast Transient Spectroelectrochemistry}

During a pump-probe measurement, the light absorbing sample (e.g., semiconductor) is excited by a short laser pulse (pump), which is followed by another light pulse (probe) to measure how the first pulse altered the absorption of the sample (Figure 15). The probe pulse should be less intense than the pump as it should not induce changes to the sample. These measurements are sensitive to changes in the ground state and excited state of a material. By varying the delay time between these two pulses, kinetic data of the absorption change can be recorded. Femtosecond transient absorption spectroscopy (TAS) allows the measurement of processes that occur on the picosecond timescale (e.g., excited state energy transfer, inter- and intramolecular electron transfer reactions) in various solvents as well as in heterogeneous systems, such as thin films. This spectroscopic method allows to simultaneously record kinetic and spectral data regarding the excited state decay of the materials of interest.

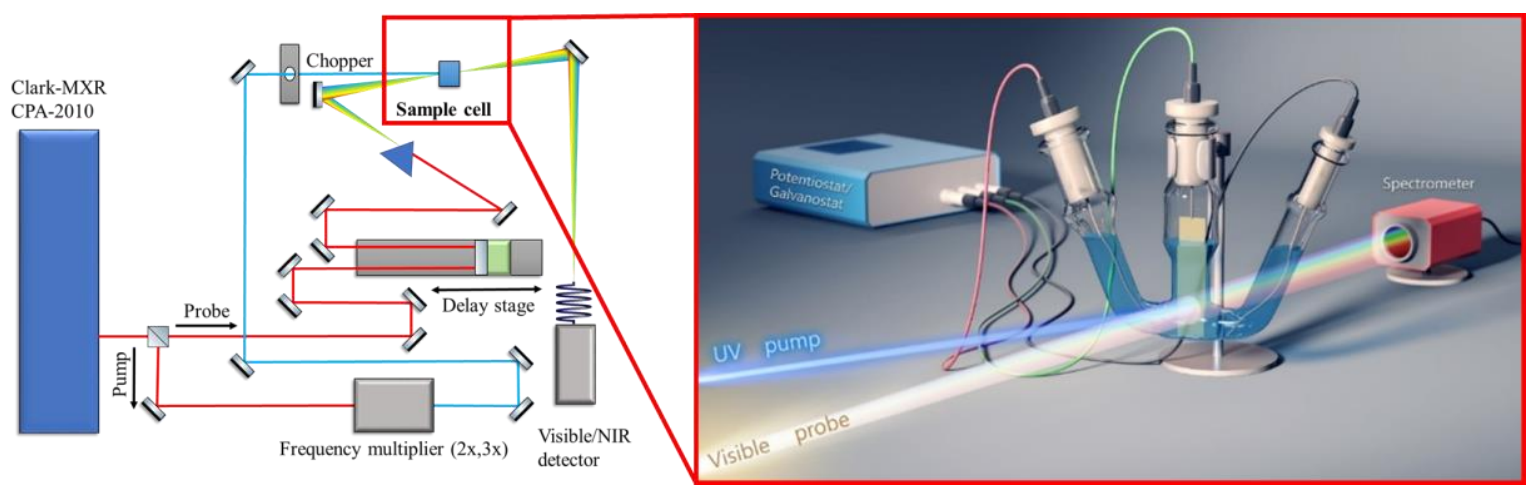

Figure 15. The used measurement setup for the ultrafast transient spectroelectrochemistry measurements.

\section{Measurement Setup}

Two key components of the system are the chopper and the delay stage. The chopper is used to remove a number of pump pulses from the pulse train at a certain frequency, which allows to probe the sample in both the excited $\left(A_{\text {pump+probe }}\right)$ and ground $\left(A_{\text {probe }}\right)$ state. The data collected is the difference between the two measurements.

$$
\Delta A=A_{\text {pump }+ \text { probe }}-A_{\text {probe }}=\log \frac{I_{\text {probe }}}{I_{\text {pump }+ \text { probe }}}
$$


The probe is redirected through the delay stage, which staggers (in time) the arrival of the pump and probe pulses at the sample. The pulse train is then sent through a Sapphire crystal to generate a white-light continuum, which defines the wavelength regime of the measurements.

Three factors limit the time-resolution and timeframe of the technique: (i) the laser pulse width, (ii) the precision of the delay stage (iii), and the length of the delay stage. The laser pulse width (150 fs in our case) is an important factor in the measurement of kinetic data. Any process that occurs on roughly the same time-scale as the pulse width will be difficult to resolve kinetically, as its excited state dynamics will be obscured within the duration of the laser pulse. The delay stage can accurately move a small enough distance to delay the pump and probe pulses by $20 \mathrm{fs}$ (a delay stage translation of about $5 \mathrm{~mm}$ ). The result of this is that the accuracy of the measurement is greater than $20 \mathrm{fs}$. The length of the delay stage is also a limiting factor in the system. The delay stage $(0.48 \mathrm{~m})$ only allows a total delay time of $1600 \mathrm{ps}$ between pump and probe arrival time in the system. With a longer delay stage measurements over a larger time window can be performed, however in most cases, a long delay stage can complicate the system setup causing the system to become impractical.

\section{Transient Absorption Spectral Features}

The recorded spectra are generally complicated as they contain contributions from several events: (i) ground state bleach, (ii) stimulated emission, and (iii) induced absorption (Figure 16A). The pump pulse excites the optically active material, which causes a ground state $\rightarrow$ excited state transition (green arrow). This results in the decrease of population in the ground state, resulting in the diminishing of the signal related to the ground state. Therefore, at the wavelength of the ground state absorption the difference absorption signal becomes negative (Figure 16B). This contribution to the absorption spectrum is called ground state bleaching (GSB). Over time this signal fades as the excited state returns to the ground state. Stimulated emission (SE) arises when the probe pulse interacts with population already in an excited state and induces their emission (red dashed arrow). This phenomenon will also show up as a negative signal on the difference absorption spectrum (Figure 16B). Because when the pump pulse interacts with the sample the detector will receive the sum of the photons from the probe and the photons emitted by the sample ( $\left.I_{\text {pump+probe }}>I_{\text {probe }}\right)$. Similarly, to fluorescence signals SE exhibits a Stokes shift (red shift compared to the absorption spectra). 
Induced absorption (IA) is the result of the excited state absorbing another photon and thus occupying an even higher excited state (blue dotted line). This can only occur in the excited state, thus it appears as a positive signal on the difference absorption spectra (Figure 16B).

A

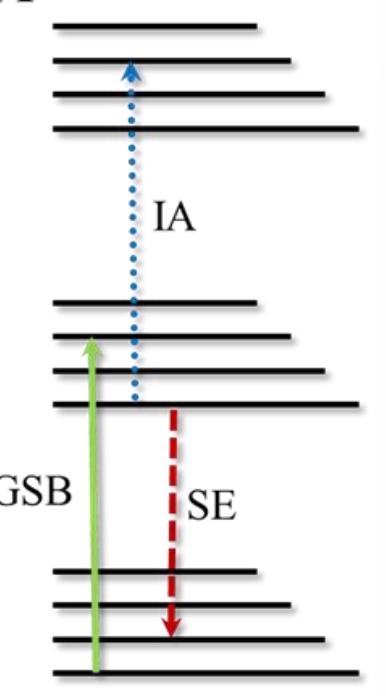

B

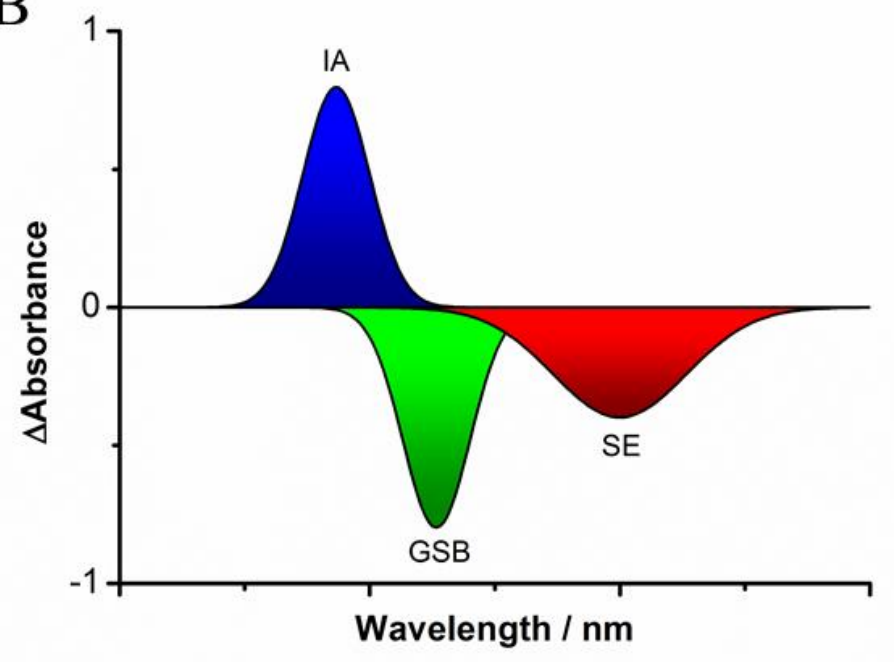

Figure 16. A: Energy levels of a hypothetical optically active sample and some quantum transitions influencing the difference absorption spectrum. B: The corresponding difference absorption spectrum with the separate contributions of the different transitions.

\section{Ultrafast-spectroelectrochemistry}

TAS measurements are usually conducted on thin film samples or solutions placed in a cuvette. However, by replacing the cuvette with an electrochemical cell, and using transparent electrodes, the measurement can be coupled with electrochemical techniques.

A Clark MXR-2010 laser system (775 nm fundamental, $1 \mathrm{~mJ} /$ pulse, FWHM=150 fs, $1 \mathrm{kHz}$ repetition rate) was used to perform the ultrafast transient spectroelectrochemistry measurements. The data was collected using a Helios software from Ultrafast Systems. The fundamental was separated in an intensity ratio of $95 / 5$, where the $95 \%$ was doubled in frequency to generate the $387 \mathrm{~nm}$ excitation pump. The remaining 5\% was used to generate a white light continuum via a $\mathrm{CaF}_{2}$ crystal. This light was used as the probe for all measurements. The intensity of the excitation pump was modulated so that the maximum change in absorbance remained constant in all measurements $\left(\Delta \mathrm{A}_{520 \mathrm{~nm}} \sim-0.25\right)$, which corresponded to a pump excitation of about $12 \mu \mathrm{J} \mathrm{cm}^{-2}$. This was done to ensure that similar amounts of charge carriers were generated in each measurement. A three-armed electrochemical cell with a fused silica cuvette body was placed at the spatial overlap of the pump and the probe. The electrode was kept at a constant potential while the TA spectra 
were recorded. The sampling spot was adjusted between every measurement to ensure the sampling of a fresh electrode area.

\subsubsection{X-ray Photoelectron Spectroscopy}

To gain information on the surface composition and the distinct chemical states of elements on the electrode surface X-ray photoelectron spectroscopy (XPS) was used. XPS is a highly surface sensitive technique, thus the information is acquired from the upper 1-10 nm region of the sample.

The XP spectra were recorded with a PHI VersaProbe II system. The binding energy scale was corrected by fixing the main $\mathrm{C} 1$ s component to $284.8 \mathrm{eV}$, corresponding to the adventitious carbon. For spectrum acquisition and evaluation, the MultiPak software was used. To fit the XP spectra and determine the elemental composition of the different samples a Shirley background was used. First the spectra of the pure $\mathrm{CsPbBr} 3$ samples were fitted. From these fittings the profile shape, position and FWHM of the fitting functions were determined. For the electrochemically treated samples these fittings functioned as starting parameters, from which the shape and FWHM was held constant for each subsequent fitting. Small variation in the peak position was allowed for each fit. If the fitting was inadequate, another component was added to the process.

\subsubsection{Scanning Electron Microscopy}

Top-down and cross-sectional scanning electron microscopic (SEM) images were captured using a FEI Helios NanoLab ${ }^{\mathrm{TM}}$ DualBeam ${ }^{\mathrm{TM}}$ instrument. Elemental composition of the samples was analysed by energy dispersive X-ray spectroscopy (EDX).

\subsubsection{X-ray Diffraction}

$\mathrm{X}$-ray diffraction (XRD) patterns were collected using a Bruker D8 DISCOVER instrument with $\mathrm{Cu} \mathrm{K} \alpha$ X-ray source $(\lambda=1.5406 \AA)$, in the $20-80^{\circ}$ range, with a $2^{\circ} \mathrm{min}^{-1}$ scan rate.

\subsubsection{Raman-spectroscopy}

Raman spectroscopic measurements were performed on a DXR Raman Microscope using a green laser $(\lambda=532 \mathrm{~nm})$, operating at $10 \mathrm{~mW}$ laser power at room temperature. 


\subsubsection{Solar Cell Characterization}

\section{j-V Curves}

A solar cell behaves in the dark like an ideal diode, namely it only lets through current in one direction. This behavior should manifest itself in the response of a solar cell in the dark. The $\mathrm{j}-\mathrm{V}$ curve of a solar cell under illumination is the superposition of the measured dark current and the generated photocurrent. Several parameters can be determined from the $\mathrm{j}-\mathrm{V}$ curve of a solar cell (Figure 17A) such as: open-circuit voltage ( $\left.\mathrm{V}_{\mathrm{OC}}\right)$, short circuit current density (jsc), fill-factor (FF), and maximum power point ( $\left.\mathrm{P}_{\mathrm{Max}}\right)$.
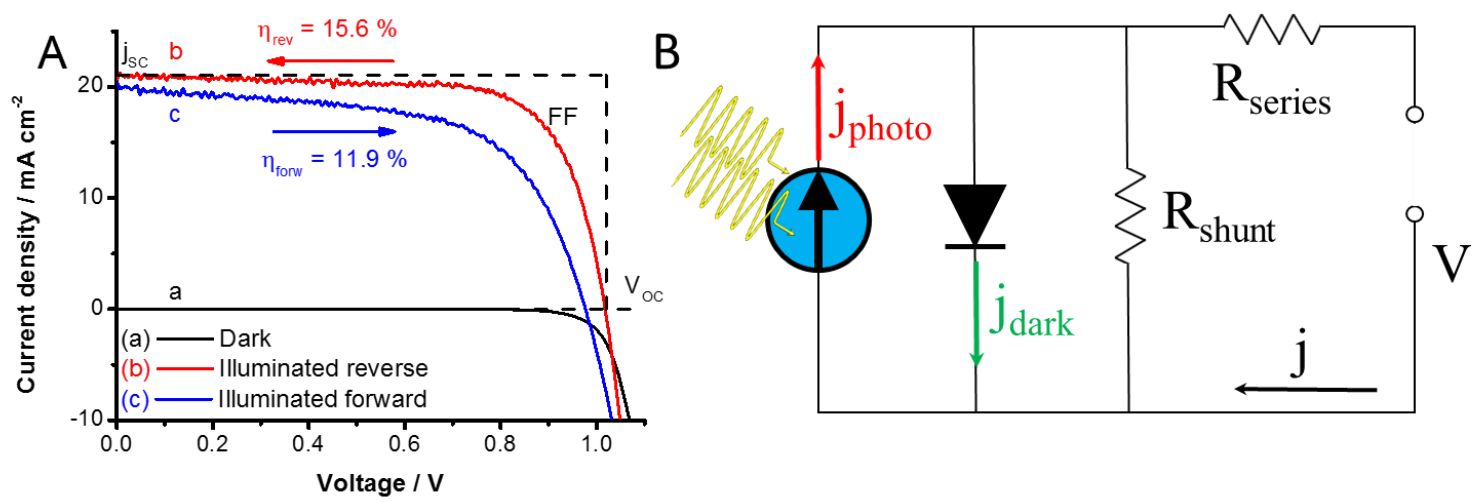

Figure 17. A: Typical j-V response of a $M A P b I_{3}$ solar cell under dark, reverse and forward sweep conditions. The sweep rate was $50 \mathrm{mV} \mathrm{s}^{-1}$ and the used illumination was 1.5 AM $100 \mathrm{~mW} \mathrm{~cm}^{-2}$. B: The equivalent circuit model of a realistic solar cell.

The jsC and the $\mathrm{V}_{\mathrm{OC}}$ are the maximum current and voltage respectively from a solar cell. The FF is defined as the ratio of the maximum power from the solar cell to the product of $\mathrm{V}_{\mathrm{OC}}$ and jsc. Graphically, the FF is a measure of the "squareness" of the j-V curve. The efficiency is the most commonly used parameter to compare the performance of one solar cell to another. It is defined as the ratio of energy output from the solar cell to input energy from the Sun. In addition to reflecting the performance of the solar cell itself, the efficiency depends on the spectrum and intensity of the incident sunlight and the temperature of the solar cell. Therefore, conditions under which efficiency is measured must be carefully controlled to compare the performance of one device to another. Terrestrial solar cells are measured under AM 1.5 conditions and at a temperature of $25^{\circ} \mathrm{C}$. From these values the efficiency of the solar cell can be derived as:

$$
\eta=\frac{P_{M a x}}{P_{I n}}=\frac{F F j_{S C} V_{O C}}{P_{I n}}
$$


The shape of a j-V curve can be indicative of parasitic losses in the cell. These resistive losses can severely distort the shape of the $\mathrm{j}-\mathrm{V}$ curve. These can be integrated into the equivalent circuit model of the solar cell (Figure 17B). One of the losses acts as a series resistance ( $\mathrm{R}_{\text {Series }}$ ). Three causes for this behavior can be identified: (i) current passing through the emitter and base of the solar cell, (ii) contact resistance between the metal contact and the solar cell, and (iii) resistance of the top and rear metal contacts. The main impact of $\mathrm{R}_{\text {Series }}$ is to reduce the FF, although excessively high values may also reduce the jsc as well. Significant power losses caused by the presence of a shunt resistance ( $\left.\mathrm{R}_{\text {Shunt }}\right)$, which is typically caused by defects in the material (recombination), rather than poor solar cell design. Low $\mathrm{R}_{\text {Shunt }}$ causes power losses in solar cells by providing an alternate current path for the light-generated current. Such a diversion reduces the amount of current flowing through the solar cell junction and reduces the voltage from the solar cell.

The characterization of the solar cells was performed in a 2-electrode setup on a Princeton Applied Research PARStat 2273 potentiostat under 1 sun conditions. The voltage was swept with a $50 \mathrm{mV} \mathrm{s}^{-1}$ sweep rate.

\section{IPCE measurement}

During an incident photon to charge carrier conversion efficiency (IPCE) measurement the current response of the solar cell is recorded in the function of the wavelength of the incident light (technically external quantum efficiency measurement). IPCE measures how efficiently the solar cell converts photons to charge carriers. Two types of quantum efficiencies can be distinguished: external and internal. The difference is that internal quantum efficiency only considers photons that are absorbed by the material and excludes photons that are reflected or transmitted at a given wavelength.

The IPCE curve can be used to determine the overall current response of a device to a given light source with known spectral distribution. By using the spectral distribution of solar light (AM $1.5100 \mathrm{~mW} \mathrm{~cm}^{-2}$ ) this should be equal to the jsc value determined from a $\mathrm{j}-\mathrm{V}$ curve (if the $\mathrm{j}-\mathrm{V}$ sweep represents the true performance characteristics of the device). A certain degree of discrepancy between the two measurements can be expected considering that IPCE measurements are carried out at a single wavelength with much lower intensity than one sun irradiation. ${ }^{[202]}$ IPCE measurements were taken with a Newport Oriel Quantum Efficiency Measurement System. 


\section{Results and Discussion}

\subsection{Establishing the Electrochemical Stability Window of Perovskite Electrodes}

\subsubsection{UV-vis Spectroscopic Studies of the Dissolution of $\mathrm{CsPbBr}_{3}$ Electrodes}

After screening a large pool of solvents and electrolytes, we concluded that DCM is a viable candidate. Most perovskites are stable in this medium and it readily dissolves commonly employed electrolytes. To probe the stability of the films, a series of UV-vis spectra were recorded in pure DCM as well as in different DCM based electrolytes for a period of $60 \mathrm{~min}$ (Figure 18). All absorbance spectra show a pronounced feature at $518 \mathrm{~nm}$ which corresponds to the excitonic transition of $\mathrm{CsPbBr}_{3}$. After an initial absorbance increase, where the film became cloudy and slightly opaque, the films began to dissolve.
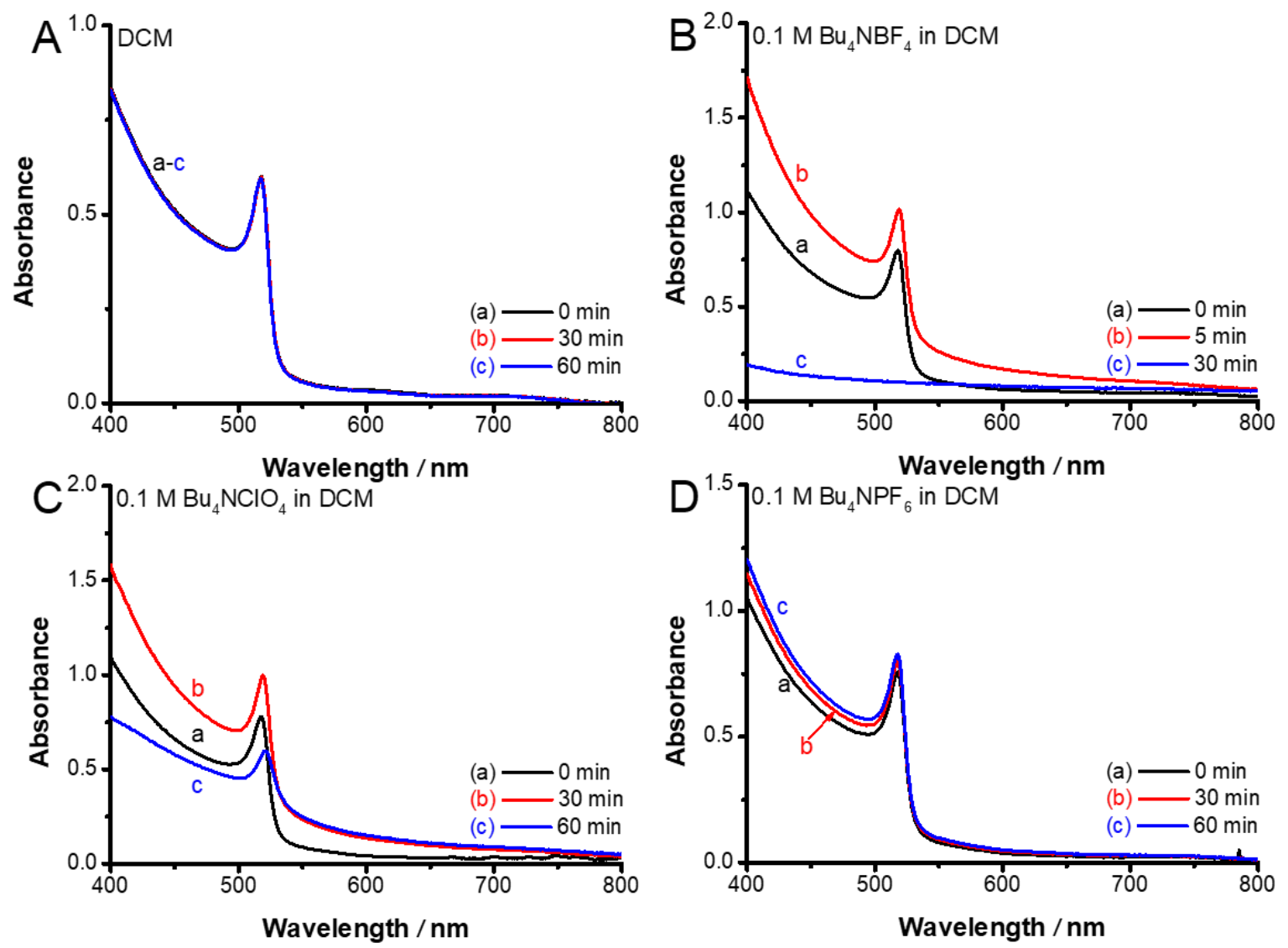

Figure 18. UV-vis absorbance spectra of $\mathrm{FTO} / \mathrm{TiO}_{2} / \mathrm{Cs} \mathrm{PbBr}_{3}$ films in different electrolytes, after different immersion times. A: pure DCM, B: $0.1 \mathrm{MBu}_{4} N B F_{4}$ in DCM, C: $0.1 \mathrm{M}$ $\mathrm{Bu}_{4} \mathrm{NClO}_{4}$ in DCM, D: $0.1 \mathrm{M} \mathrm{Bu}_{4} \mathrm{NPF}_{6}$ in DCM.

The normalized absorbance traces, measured at $518 \mathrm{~nm}$, are compared in Figure 19 for the different electrolytes. The rate of dissolution is related to the complexing ability of 
the anions as well as the specific interactions between the different anions in the solution and the cations in the perovskite lattice. ${ }^{[203,204]}$ The following definite trend was found in the dissolution rate: $\mathrm{BF}_{4}^{-}>\mathrm{ClO}_{4}^{-}>{\mathrm{PF}_{6}}^{-}$. Based on these observations, we have chosen $\mathrm{Bu}_{4} \mathrm{NPF}_{6}$ for all further experiments. We note here that even trace amounts of water can completely ruin the stability of these samples (in our experiments the water content was always kept below 10 ppm). Therefore, electrochemical experiments involving lead halide perovskites should be conducted in an inert environment. This can either be achieved by performing measurements inside a glove box or inside a sealed electrochemical cell that has been thoroughly purged. The latter option was used for all experiments discussed in my dissertation. Additionally, the water content of both the pure solvents and the solutions needs to be checked (e.g., by Karl Fischer titrations) and additional drying steps should be employed if necessary.

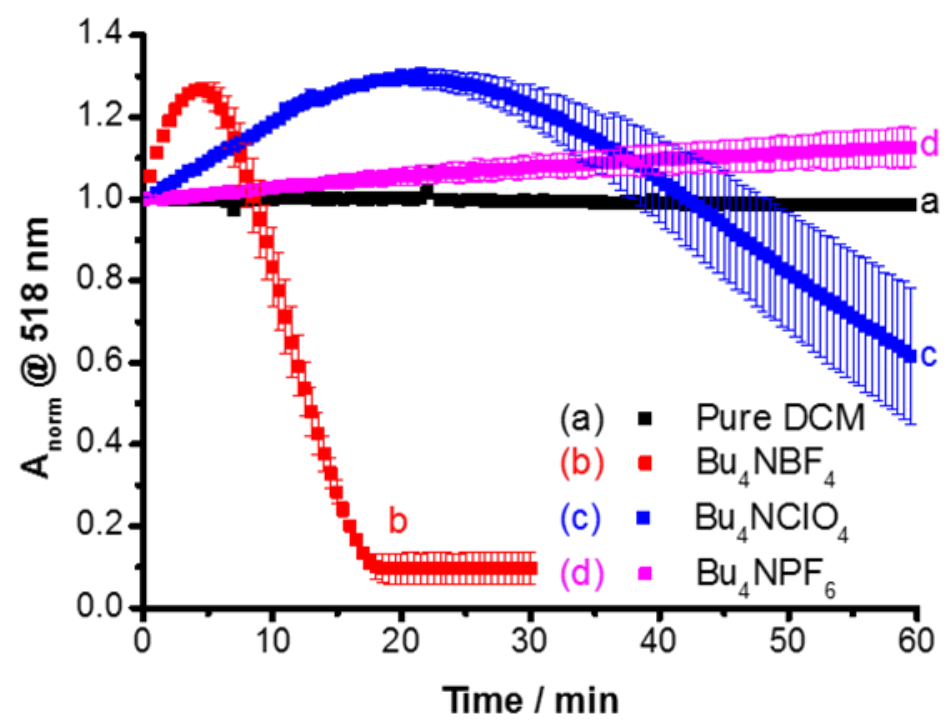

Figure 19. Normalized absorbance change at $518 \mathrm{~nm}$ of $\mathrm{FTO}_{\mathrm{TiO}} / \mathrm{Cs}_{\mathrm{PbBr}}$ electrodes in different DCM based electrolytes. The error bars were derived from measurements on three separate films.

\subsubsection{Spectroelectrochemistry of $\mathrm{CsPbBr} 3$ Electrodes}

In the next step, two separate spectroelectrochemical experiments were carried out, where the potential was scanned from the open circuit potential (OCP) value to both anodic (Figure 20) and cathodic directions (Figure 22). 

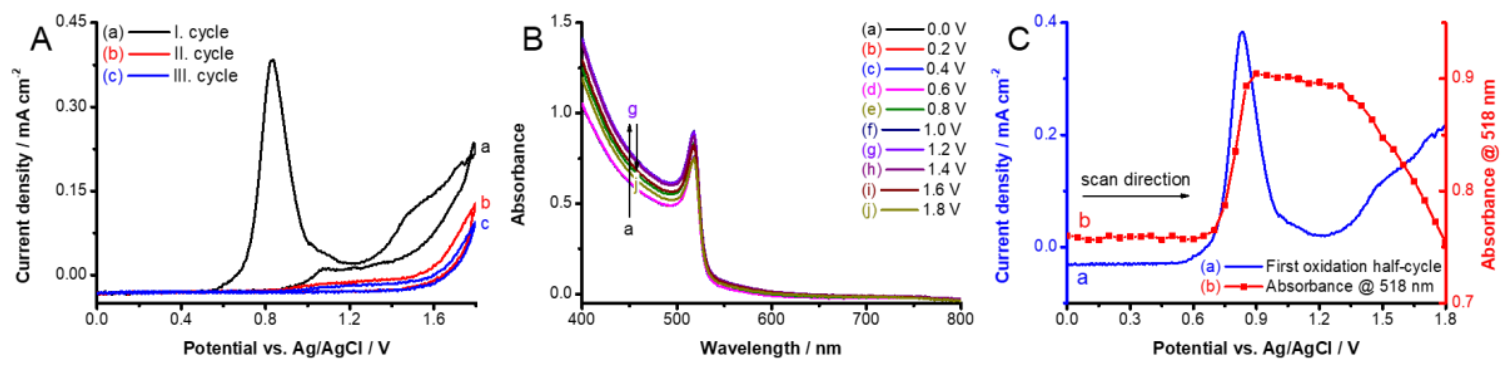

Figure 20. Spectroelectrochemical data, recorded for $\mathrm{FTO} / \mathrm{TiO}_{2} / \mathrm{Cs}_{\mathrm{PbBr}}$ films in $0.1 \mathrm{M}$ $B u_{4} N P F_{6} / D C M$ electrolyte (10 $\mathrm{mV} \mathrm{s}^{-1}$ sweep rate). A: Three subsequent $C V$ curves recorded during oxidation $\boldsymbol{B}: U V$-vis spectra recorded at various potentials during oxidation. $\boldsymbol{C}$ : The first oxidation half cycle plotted together, with the absorbance change at the excitonic peak.

Under positive (anodic) bias, the first oxidation wave was situated at $+0.8 \mathrm{~V}$, followed by a second oxidation peak starting at $+1.3 \mathrm{~V}$. In fact, this second wave is a sum of two peaks, as seen from the shoulder in Figure 20A. These redox events are reflected in distinct changes that occur in the optical behavior shown in Figure 20B. To tie the optical changes to redox events the first oxidation half-cycle was plotted together with the absorbance change of the excitonic peak (Figure 20C). During oxidation, the absorbance increased below $530 \mathrm{~nm}$ in the first oxidation step with the onset of $\sim+0.6 \mathrm{~V}$. The absorbance drastically decreased during the second step (onset $\sim+1.3 \mathrm{~V}$ ). The shape of the initial absorbance increase (i.e., difference absorbance spectrum) mirrors the initial spectrum of the electrode (Figure 21A) which indicates that the perovskite structure is preserved in the film even with a surface etching phenomenon occurring which causes the increase in absorbance (Figure 21C). In addition, the absorbance increase (and opacity) witnessed upon immersion into $0.1 \mathrm{M} \mathrm{Bu}_{4} \mathrm{NPF}_{6}$ is also very similar (Figure 21B). Overall, we think that this absorbance increase is not rooted in the formation of a new material, but in other physical factors (e.g., scattering) which enhances the absorption of the perovskite film. ${ }^{[205]}$
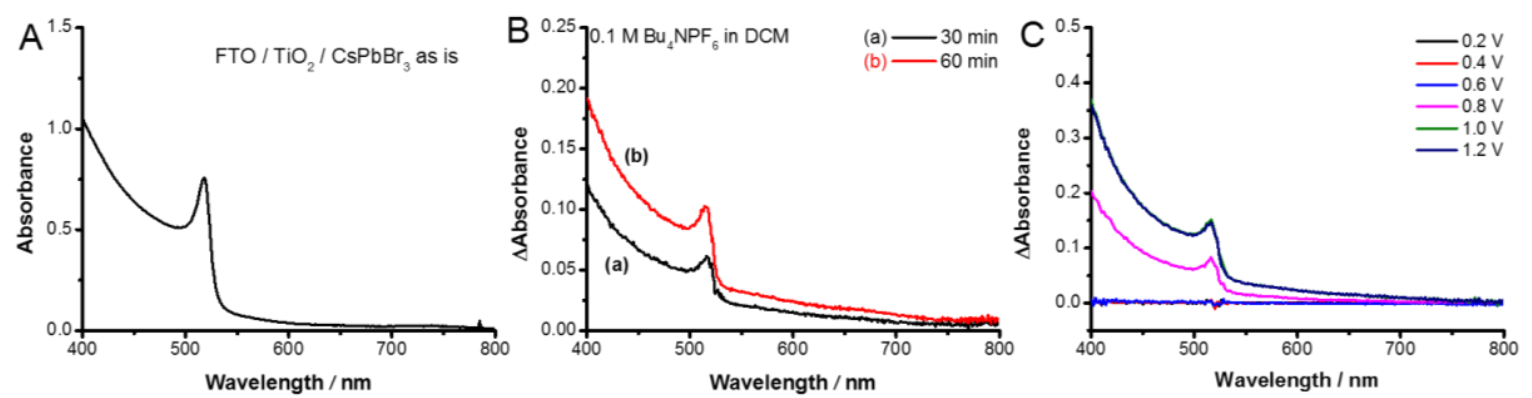

Figure 21. A: UV-vis absorbance spectrum of a $\mathrm{FTO}_{\mathrm{TiO}} / \mathrm{CsPbBr}_{3}$ film in $0.1 \mathrm{M}$ $B u_{4} N P F_{6}$. Difference spectra after $\boldsymbol{B}$ : different immersion times and $\boldsymbol{C}$ : oxidation treatment. 
As for the reduction, there was a single redox peak centered at $-1.4 \mathrm{~V}$, where notably higher current densities were measured compared to the oxidative events (Figure 22A). As a result of the reduction, there was a rapid decrease in the absorbance at the excitonic peak characteristic to the perovskite material (Figure 22B and C). In parallel, there was an increased non-characteristic absorption in the whole wavelength range, which was also confirmed by the reflective metallic appearance of the film (Figure 23).
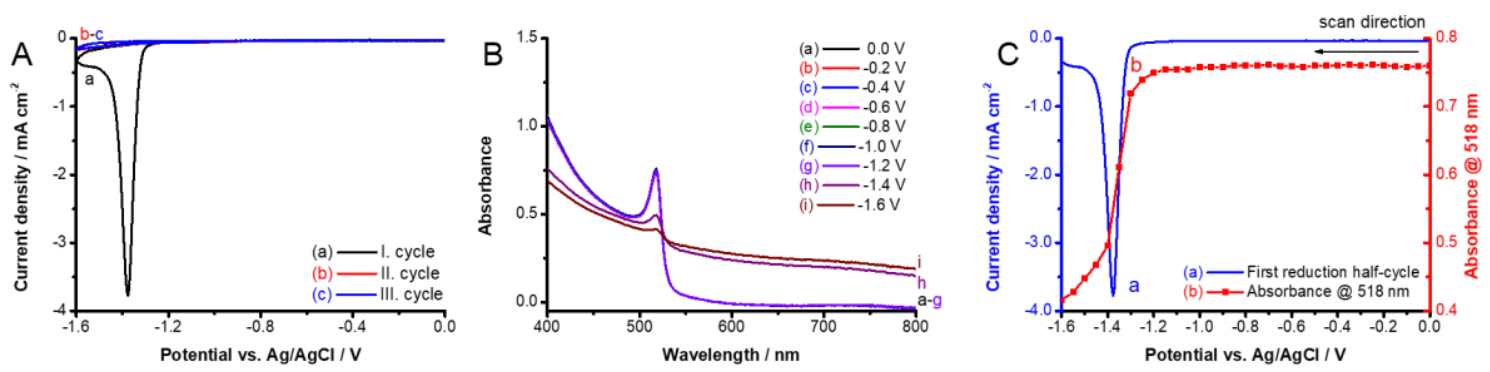

Figure 22. Spectroelectrochemical data, recorded for $\mathrm{FTO}_{\mathrm{TiO}} / \mathrm{Cs}_{\mathrm{PbBr}}$ films in $0.1 \mathrm{M}$ $B u_{4} N P F_{6} / D C M$ electrolyte $\left(10 \mathrm{mV} \mathrm{s}^{-1}\right.$ sweep rate). A: Three subsequent $C V$ curves recorded during reduction $\boldsymbol{B}: U V$-vis spectra recorded at various potentials during reduction. $C$ : The reduction half cycle plotted together, with the absorbance change at excitonic peak.
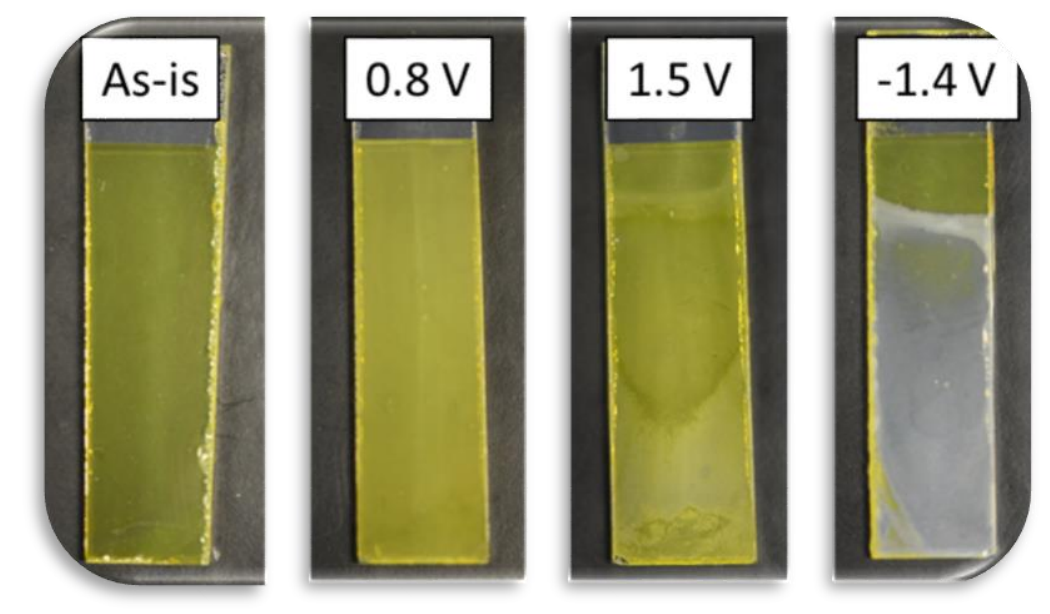

Figure 23. Photographs of $\mathrm{FTO} / \mathrm{TiO}_{2} / \mathrm{Cs}_{\mathrm{SbBr}}$ electrodes treated at selected potential values for $30 \mathrm{~s}$.

\subsubsection{Chemical Composition of Reduced/Oxidized CsPbBr 3 Electrode Surfaces}

The question naturally comes, whether the peaks on the cyclic voltammograms in Figure 20A and Figure 22A correspond to Faradaic events (i.e., reduction of ions) or they are simply related to electron and hole injection into the CB and VB, respectively; and thus, represent band energy values. To probe the possible changes in the $\mathrm{CsPBr}_{3}$ structure, the electrodes were polarized at $E=-1.4 \mathrm{~V},+0.8 \mathrm{~V}$, and $+1.5 \mathrm{~V}$ for $30 \mathrm{~s}$ in $0.1 \mathrm{M} \mathrm{Bu}_{4} \mathrm{NPF}_{6} / \mathrm{DCM}$ 
solution. After carefully washing the electrodes with DCM and drying them in an Argon stream, XPS measurements were carried out to analyze the chemical composition of the surface. Several important trends can be revealed by the careful analysis of the XP spectra (Figure 24): (i) the intensity of the $\mathrm{Br}^{-}$-related signal decreased during all treatments and a new species developed during the reduction; (ii) the $\mathrm{F}^{-}$signal emerged upon oxidation and this trend was more pronounced for samples treated at more positive potentials; (iii) in parallel, a new electron-rich form of $\mathrm{Cs}^{+}$developed for the oxidized samples; and (iv) a new form of oxygen arose in the reduced sample (in addition to the adsorbed oxygen moieties).
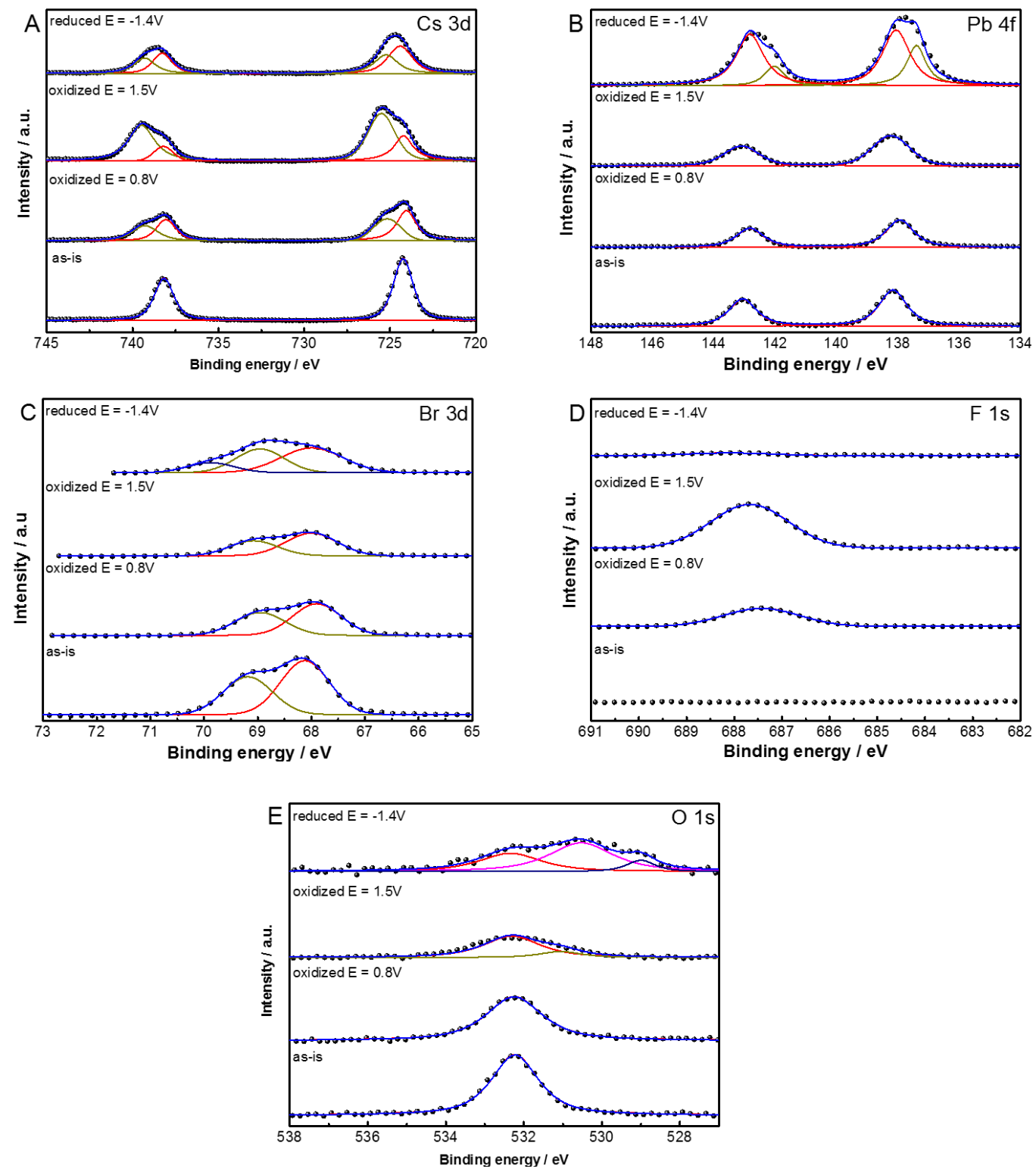

Figure 24. High resolution XPS data for the $\mathrm{FTO}_{\mathrm{TiO}} / \mathrm{Cs}_{\mathrm{PbBr}}$ electrodes treated at selected potential values (as-is, $-1.4 \mathrm{~V},+0.8 \mathrm{~V},+1.5 \mathrm{~V}$ ), for $30 \mathrm{~s}$ in $0.1 \mathrm{MBu} \mathrm{B}_{4} \mathrm{~F}_{6} \mathrm{DCM}$ solution. A: Cs $3 d, \boldsymbol{B}: \mathrm{Pb} 4 f, \boldsymbol{C}: \mathrm{Br} 3 d, \mathrm{D}: \mathrm{F} 1 \mathrm{~s}, \boldsymbol{E}: \mathrm{O} 1 \mathrm{~s} X \mathrm{P}$ spectrum. 
Taking these trends together as a whole, we propose the following explanations. The obvious reduction reaction is the formation of $\mathrm{Pb}$ at negative potentials (as also confirmed by $\mathrm{SEM}-\mathrm{EDX}$ analysis, shown later). $\mathrm{Pb}$ is partially re-oxidized to $\mathrm{PbO}$ upon air exposure while preparing the electrode for XPS measurements, confirmed by both the $\mathrm{Pb}$ and $\mathrm{O}$ signals. During oxidation, the $\mathrm{Br}^{-}$and $\mathrm{Pb}^{2+}$ content is gradually lost due to the destruction of the $\mathrm{CsPBr}_{3}$ structure. In parallel, the $\mathrm{F}^{-}$content (with a binding energy typical for $\mathrm{PF}_{6}^{-}$) increased significantly, suggesting the formation of $\mathrm{CsPF}_{6}$ on the surface. This notion was further confirmed by SEM-EDX, which proved the presence of $\mathrm{P}$ in the oxidized samples. These trends are quantified in Table 3, where the surface composition of the various samples is presented. The larger dispersion in the case of the reduced electrodes further confirmed the inhomogeneity of these samples, to be shown later on the SEM images.

Table 3. Compositional analysis of the surface of the electrochemically treated $\mathrm{FTO} / \mathrm{TiO}_{2} / \mathrm{CsPbBr}_{3}$ electrodes using XPS. The standard deviation values were derived from analyzing XP spectra captured from three different spots of the sample.

\begin{tabular}{|c|c|c|c|c|}
\hline Sample & Br $/ \mathbf{a t} \%$ & $\mathrm{Cs} / \mathbf{a t} \%$ & $\mathbf{P b} / \mathbf{a t} \%$ & $\mathbf{F} / \mathbf{a t} \%$ \\
\hline As-is & $59.9 \pm 0.6$ & $22.6 \pm 1.0$ & $17.5 \pm 0.4$ & 0 \\
\hline Reduced (-1.4 V) & $40.7 \pm 2.7$ & $20.6 \pm 0.8$ & $26.9 \pm 5.7$ & $11.8 \pm 7.5$ \\
\hline Oxidized (0.8 V) & $22.2 \pm 1.8$ & $15.9 \pm 1.0$ & $8.4 \pm 0.7$ & $53.6 \pm 2.1$ \\
\hline Oxidized (1.5 V) & $9.6 \pm 1.0$ & $14.3 \pm 0.5$ & $5.2 \pm 0.9$ & $71.0 \pm 1.5$ \\
\hline
\end{tabular}

To further quantify the above-mentioned trends, we deconvoluted the spectra in Figure 24, which allowed for quantitative analysis of the surface composition, and specifically to determine the amount of the various species. For example, upon the oxidation of $\mathrm{CsPbBr}_{3}$, we assume the formation of $\mathrm{CsPF}_{6}$. By analyzing the $\mathrm{Cs} 3 \mathrm{~d}$ region, two distinct species were identified. By assigning the ones at $738.3 \mathrm{eV}$ and $724.3 \mathrm{eV}$ to $\mathrm{CsPbBr}_{3}$ and the ones $739.6 \mathrm{eV}$ and $725.2 \mathrm{eV}$ to $\mathrm{CsPF}_{6}$, we calculated the theoretical $\mathrm{Br}^{-}, \mathrm{F}^{-}$and $\mathrm{Pb}^{2+}$ content (Table 4). As seen in Table 4, a very good match was found between the measured and calculated data. 
Table 4. Compositional analysis of the surface of the $\mathrm{FTO} / \mathrm{TiO}_{2} / \mathrm{Cs}_{\mathrm{PbBr}}$ electrodes oxidized at selected potential values using XPS.

\begin{tabular}{|c|c|c|c|c|c|c|c|c|c|c|c|}
\hline Sample & $\begin{array}{c}\text { Total Cs } \\
\text { content } \\
\text { / at\% }\end{array}$ & $\begin{array}{c}\text { Peak ratio } \\
\text { for } \text { SSPF }_{6} \\
(739.3 \mathrm{eV})\end{array}$ & $\begin{array}{c}\text { Peak ratio } \\
\text { for } \text { CsPF }_{6} \\
(725.2 \mathrm{eV})\end{array}$ & $\begin{array}{l}\text { Average } \\
\mathrm{CsPF}_{6} / \%\end{array}$ & $\begin{array}{c}\mathrm{CsPF}_{6} / \\
\text { at\% }\end{array}$ & $\begin{array}{c}\text { Calculated } \\
\text { F/ at } \%\end{array}$ & $\begin{array}{l}\text { Measured } \\
\text { F / at\% }\end{array}$ & $\begin{array}{l}\text { Calculated } \\
\mathrm{Br} / \mathrm{at} \%\end{array}$ & $\begin{array}{c}\text { Measured } \\
\mathrm{Br} / \text { at } \%\end{array}$ & $\begin{array}{l}\text { Calculated } \\
\mathrm{Pb} / \text { at } \%\end{array}$ & $\begin{array}{c}\text { Measured } \\
\mathrm{Pb} / \mathrm{at} \%\end{array}$ \\
\hline $\begin{array}{c}\text { Oxidized } \\
(\mathrm{E}=0.8 \mathrm{~V})\end{array}$ & 15.9 & $48.8 \%$ & $41.2 \%$ & 45.0 & 7.2 & 42.9 & 53.6 & 26.1 & 22.2 & 8.7 & 8.4 \\
\hline $\begin{array}{l}\text { Oxidized } \\
(\mathrm{E}=1.5 \mathrm{~V})\end{array}$ & 14.3 & $81.2 \%$ & $66.7 \%$ & 73.9 & 10.6 & 63.4 & 71 & 11.1 & 9.6 & 3.7 & 5.2 \\
\hline
\end{tabular}

\subsubsection{Structural Changes of Reduced/Oxidized CsPbBr3 Electrodes}

To further confirm the above conclusions, XRD analysis was also performed. We note that we consider XPS analysis to be more informative, due to the very small thickness of the films employed in this study. In addition, because of the complexity of the system, and the large number of possible corrosion products, there are notable overlaps among the various diffractions. Because of these two factors, we only make qualitative conclusions, which are in line with the XPS data presented above. Figure 25 shows the XRD patterns of $\mathrm{FTO} / \mathrm{TiO}_{2} / \mathrm{CsPbBr}_{3}$ electrodes, held at the same potential values as those presented in Figure 24.
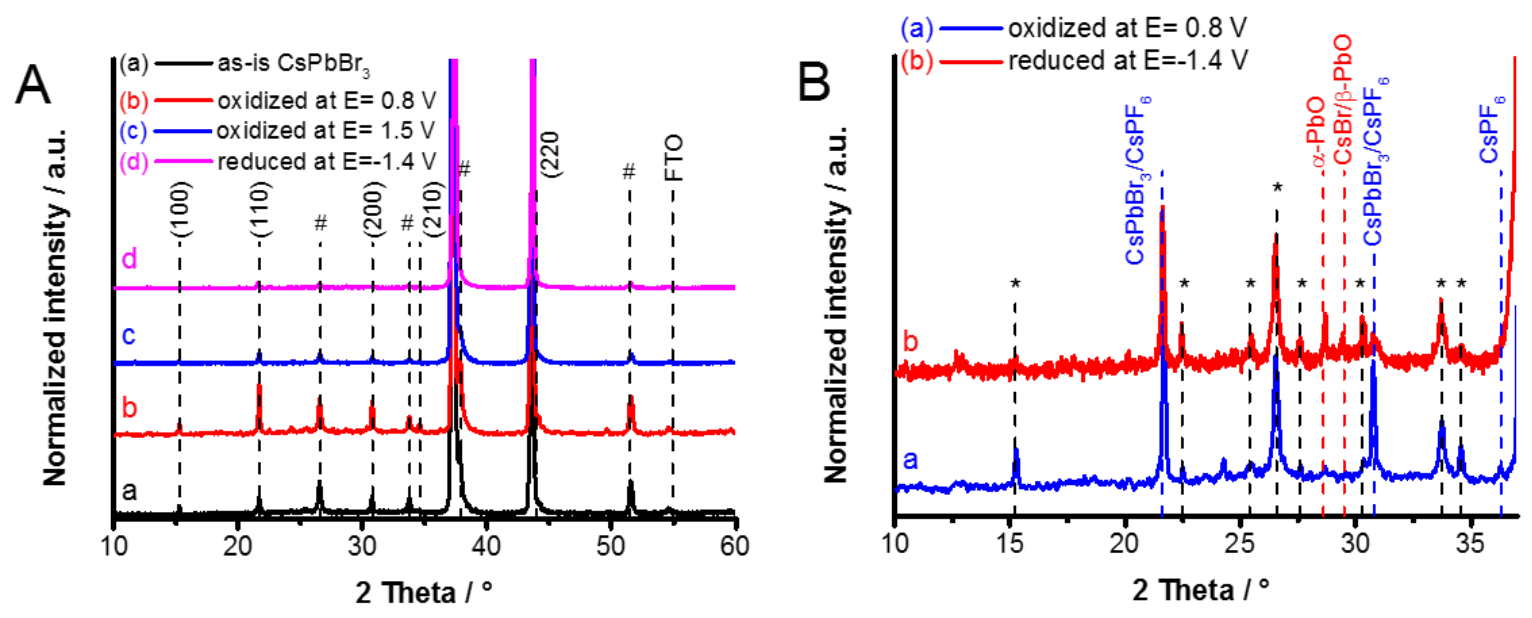

Figure 25. A: XRD patterns for the $\mathrm{FTO} / \mathrm{TiO}_{2} / \mathrm{CsPbBr}_{3}$ electrodes treated at selected potential values (as is, $-1.4 \mathrm{~V},+0.8 \mathrm{~V},+1.5 \mathrm{~V}$ ) for $30 \mathrm{~s}$ in $0.1 \mathrm{M} \mathrm{Bu} 4 \mathrm{NPF} 6 / \mathrm{DCM}$ solution. The peaks marked with \# represent overlapping diffractions of $\mathrm{CsPbBr}_{3}$ and the $\mathrm{FTO} / \mathrm{TiO}_{2}$ substrate. B: Magnified regions of the diffraction patterns of the samples held at $-1.4 \mathrm{~V}$ and $+0.8 \mathrm{~V}$. The peaks marked with * belong to the untreated $\mathrm{FTO} \mathrm{TiO}_{2} / \mathrm{Cs}_{\mathrm{PbBr}}$ electrodes.

The characteristic diffraction peaks of $\mathrm{CsPbr}_{3}$ were identified on all diffractograms at $15.3^{\circ}, 21.7^{\circ}, 26.6^{\circ}, 30.8^{\circ}$ and $33.8^{\circ} 2$ theta values. ${ }^{[78]}$ Upon oxidation, a gradual decrease was witnessed in the intensity of these diffractions (Figure 25A), although because of the overlap of several diffractions this is not trivial for the first view. In addition, low intensity 
diffractions related to $\mathrm{CsPF}_{6}$ developed at 2 theta $=21.6^{\circ}, 30.7^{\circ}$ and $36.4^{\circ}$ (PDF: 00-0340506). Note that the elemental composition, deduced from XPS data, semi-quantitatively confirmed the formation of $\mathrm{CsPF}_{6}$. A substantial decrease in the intensity of the diffractions associated with $\mathrm{CsPbr}_{3}$ was seen as a result of the cathodic treatment. In addition, $\mathrm{PbO}$ related diffraction peaks appeared (PDF: 00-085-1288), suggesting that the formed $\mathrm{Pb}$ is readily re-oxidized to $\mathrm{PbO}$ upon air exposure. ${ }^{[206]}$ Based on the above observations, the plausible reactions (12)-(14) are summarized below:

Oxidation:

$$
\mathrm{CsPbBr}_{3} \rightarrow \mathrm{PbBr}_{2}+\mathrm{Cs}^{+}+0.5 \mathrm{Br}_{2}+e^{-}
$$

$$
\mathrm{PbBr}_{2} \rightarrow \mathrm{Pb}^{2+}+\mathrm{Br}_{2}+2 e^{-}
$$

Reduction:

$$
\mathrm{CsPbBr}_{3}+2 e^{-} \rightarrow \mathrm{Pb}+\mathrm{Cs}^{+}+3 \mathrm{Br}^{-}
$$

To validate that the observed chemical changes are a direct consequence of the redox events identified on the voltammetric scans (Figure 20A and Figure 22A), control measurements were carried out at milder conditions, of which $-0.4 \mathrm{~V},+0.4 \mathrm{~V}$, and simple immersion (i.e., no electrical bias was employed) were chosen. As seen in Figure 26, there was no change when the electrode was placed into the solution and when only moderate bias was employed. Most importantly, only negligible amounts of $\mathrm{F}^{-}$was detected (due to physisorbed $\mathrm{PF}_{6}^{-}$), and the $\mathrm{Br}, \mathrm{Cs}, \mathrm{Pb}$, and $\mathrm{O}$ elements were of identical chemical nature to those in the pristine sample (Figure 26). 

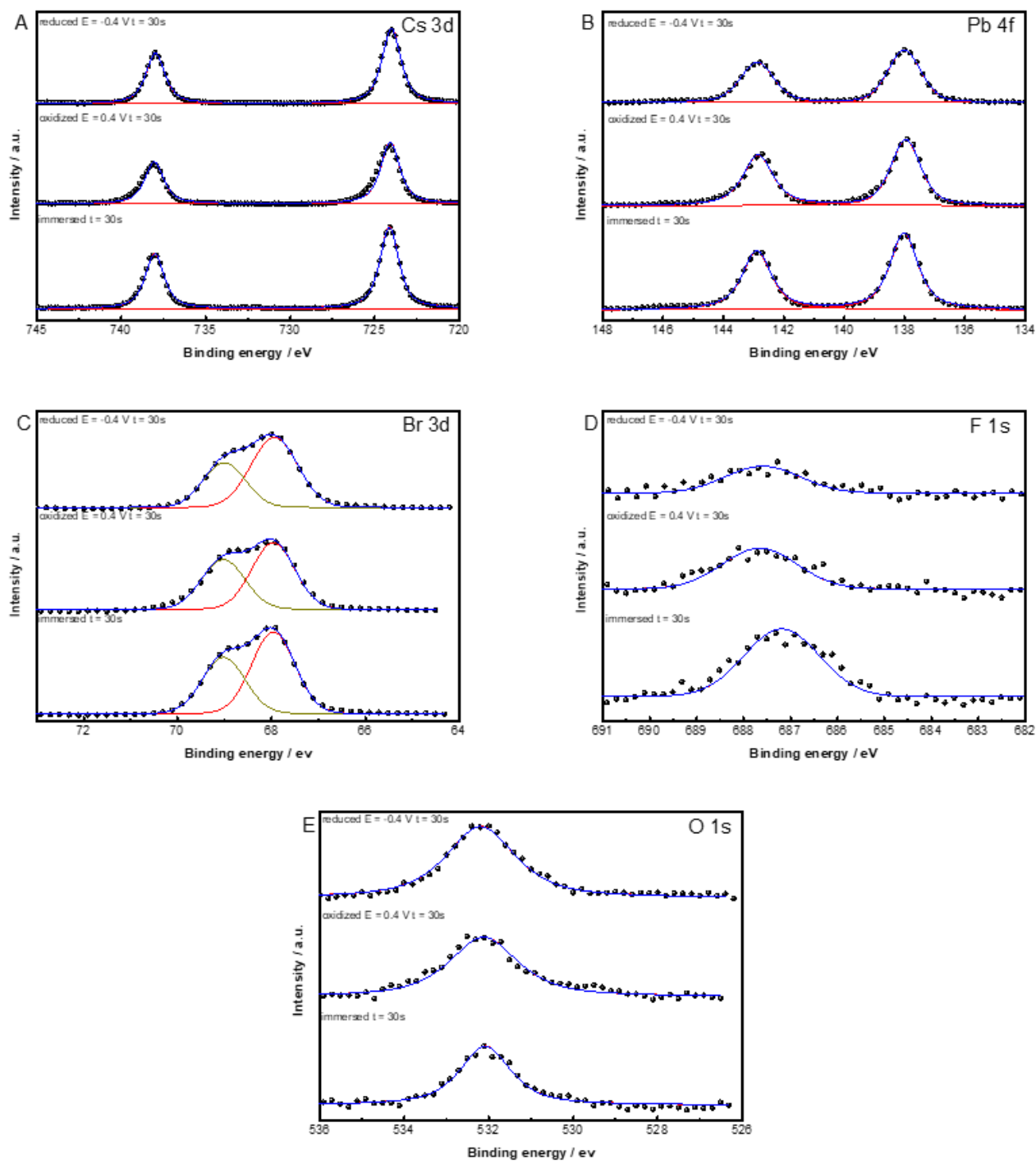

Figure 26. High resolution XPS data for the $\mathrm{FTO} / \mathrm{TiO}_{2} / \mathrm{Cs} \mathrm{PbBr}_{3}$ electrodes, treated at selected potential values $(O C P,-0.4 \mathrm{~V}$, and $+0.4 \mathrm{~V})$ for $30 \mathrm{~s}$ in $0.1 \mathrm{M} \mathrm{Bu} \mathrm{N}_{\mathrm{NPF}} \mathrm{F} D \mathrm{DCM}$ solution. A: Cs 3d, B: $\mathrm{Pb} 4 f, \boldsymbol{C}: \mathrm{Br} 3 d, \mathrm{D}: \mathrm{F} 1 \mathrm{~s}, \boldsymbol{E}: \mathrm{O} 1 \mathrm{~s} X \mathrm{P}$ spectrum.

The quantitative surface composition analysis also supported these qualitative observations (Table 5).

Table 5. Compositional analysis of the surface of the mildly treated $\mathrm{FTO} / \mathrm{TiO}_{2} / \mathrm{CsPbBr}_{3}$ electrodes using XPS.

\begin{tabular}{|c|c|c|c|c|}
\hline Sample & Br $/ \mathbf{a t} \%$ & $\mathbf{C s} / \mathbf{a t} \%$ & $\mathbf{P b} / \mathbf{a t} \%$ & $\mathbf{F} / \mathbf{a t} \%$ \\
\hline Immersed & $53.3 \pm 1.2$ & $23.6 \pm 1.6$ & $16.3 \pm 0.7$ & $6.9 \pm 1.9$ \\
\hline Oxidized (0.4 V) & $52.8 \pm 0.4$ & $21.6 \pm 0.8$ & $16.6 \pm 1.6$ & $9.0 \pm 1.1$ \\
\hline Reduced (-0.4 V) & $56.7 \pm 0.6$ & $24.0 \pm 0.1$ & $16.4 \pm 0.8$ & $3.0 \pm 1.5$ \\
\hline
\end{tabular}




\subsubsection{Morphological Changes of Reduced/Oxidized CsPbBr3 Electrodes}

Morphological changes that occurred during the redox events were visualized using SEM and SEM-FIB (to capture side-view images). The pristine sample adopts a granular morphology typically seen for $\mathrm{CsPbrr}_{3}$ and it remains almost intact under mild electrochemical conditions (Figure 27).
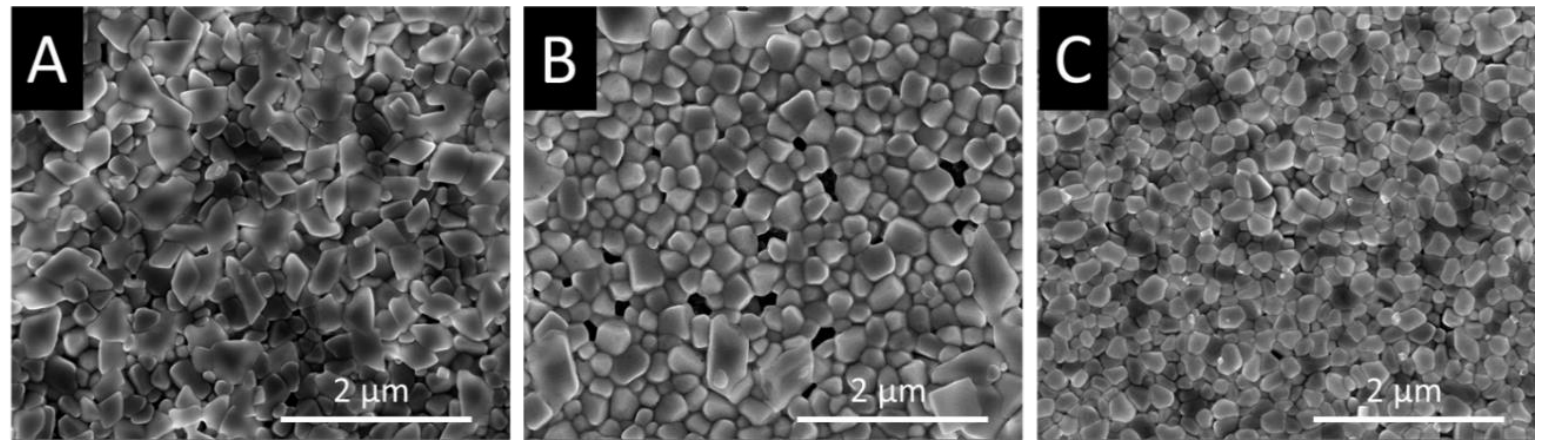

Figure 27. SEM images for the $\mathrm{FTO} / \mathrm{TiO}_{2} / \mathrm{Cs}_{\mathrm{PbBr}}$ electrodes treated at selected potential values $(\mathrm{A}: \mathrm{OCP}, \mathrm{B}:+0.4 \mathrm{~V}$, and $\mathrm{C}:-0.4 \mathrm{~V})$ for $30 \mathrm{~s}$ in $0.1 \mathrm{M} \mathrm{Bu} 4 \mathrm{NPF} / \mathrm{DCM}$ solution.

The top view and cross-section images are compiled in Figure 28. When the electrodes were exposed to more extreme electrochemical conditions (under the same circumstances as the samples discussed above), notable changes happened. Upon reduction, the particulate morphology disappeared, and a compact film was formed at certain areas of the electrode (Figure 28B and Figure 29). At lower magnification, we could even observe formation of dendrites. EDX analysis confirmed that the dendrites are made of $\mathrm{Pb}$ (Figure 29). During oxidation, rectangular particles were formed, which grew with the increasing potential (Figure 28C and D). For the sample held at $+1.5 \mathrm{~V}$ bias potential, the underlying mesoporous $\mathrm{TiO}_{2}$ became visible because of the destruction of the original perovskite film (Figure 28D). What is common in all cases is that the initial compact film turned into a porous one. Thus, the applied potential impacts the morphology to a different extent (Figure 28E-H). The side-view images provide similar insights: (i) the $\mathrm{CsPbBr}_{3}$ disappears at certain areas and the $\mathrm{TiO}_{2}$ becomes visible, (ii) a hollow structure is formed upon oxidation (Figure 28G and $\mathbf{H}$ ), due to the dissolution of the perovskite. 

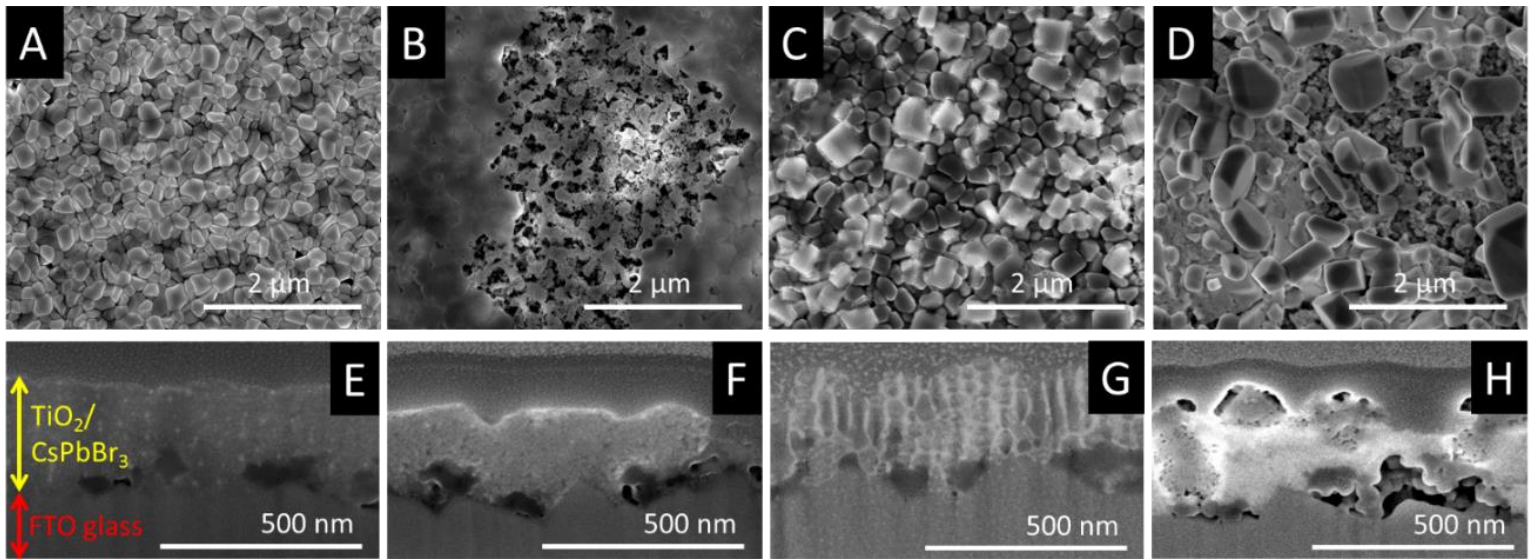

Figure 28. SEM images of $\mathrm{Cs} P b B r_{3}$ films both from top and side views. $\boldsymbol{A}, \boldsymbol{E}$ : pristine, $\boldsymbol{B}$, $\boldsymbol{F}:$ reduced at $\mathrm{E}=-1.4 \mathrm{~V}, \boldsymbol{C}, \boldsymbol{G}:$ oxidized at $\mathrm{E}=+0.8 \mathrm{~V}, \boldsymbol{D}, \boldsymbol{H}:$ oxidized at $E=+1.4 \mathrm{~V}$. All treatments lasted $30 \mathrm{~s}$ in $0.1 \mathrm{M} \mathrm{Bu} 4 \mathrm{NPF} / \mathrm{DCM}$ solution.

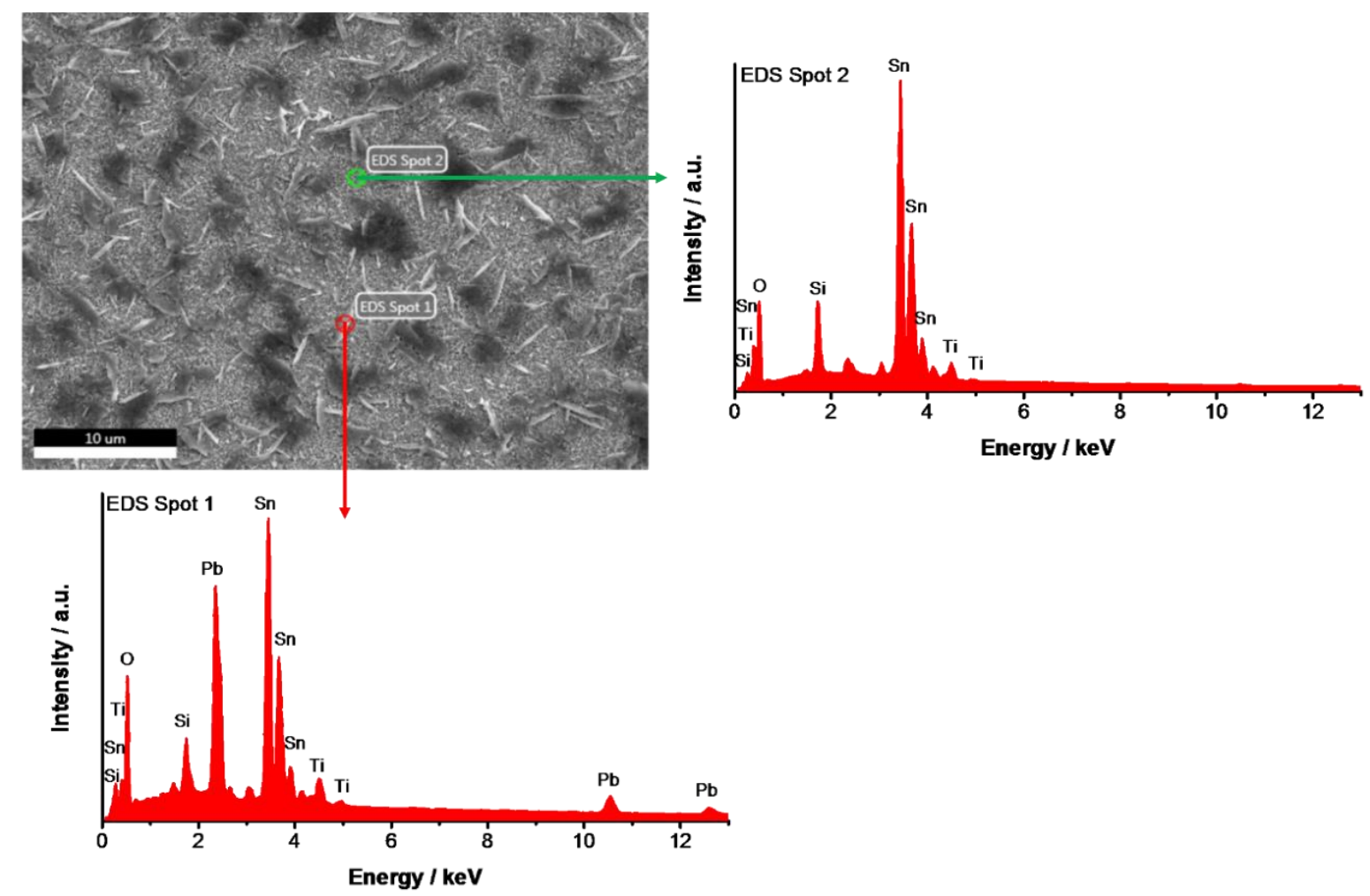

Figure 29. SEM image and EDX data recorded for a $\mathrm{FTO} / \mathrm{TiO}_{2} / \mathrm{CsPbBr}_{3}$ electrode treated at $E=-1.4 \mathrm{~V}$ for $30 \mathrm{~s}$ in $0.1 \mathrm{M} \mathrm{Bu} \mathrm{NPF}_{6} / D C M$ solution.

\subsubsection{Spectroelectrochemistry of $\mathrm{PbBr} 2$ Electrodes}

To better understand the nature of these redox transformations, control experiments were carried out with $\mathrm{FTO} / \mathrm{TiO}_{2}$ and $\mathrm{FTO} / \mathrm{TiO}_{2} / \mathrm{PbBr}_{2}$ films (Figure 30). The $\mathrm{PbBr}_{2}$ was more resistant towards oxidation, as the $\mathrm{FTO} / \mathrm{TiO}_{2} / \mathrm{PbBr}_{2}$ was stable up to $+1.6 \mathrm{~V}$ (vs. $+0.7 \mathrm{~V}$ in the case of $\mathrm{CsPBr}_{3}$ ). This suggests that only the third oxidation peak in Figure $20 \mathrm{~A}$ is related to either $\mathrm{PbBr}_{2}$ or the substrate, while the other two waves (at less positive potentials) 
are characteristic to $\mathrm{CsPbBr}_{3}$. As for the reduction, a more interesting trend was seen: the $\mathrm{CsPbBr}$, was more stable than $\mathrm{PbBr}_{2}$, as this latter was reduced at $450 \mathrm{mV}$ less negative potential.
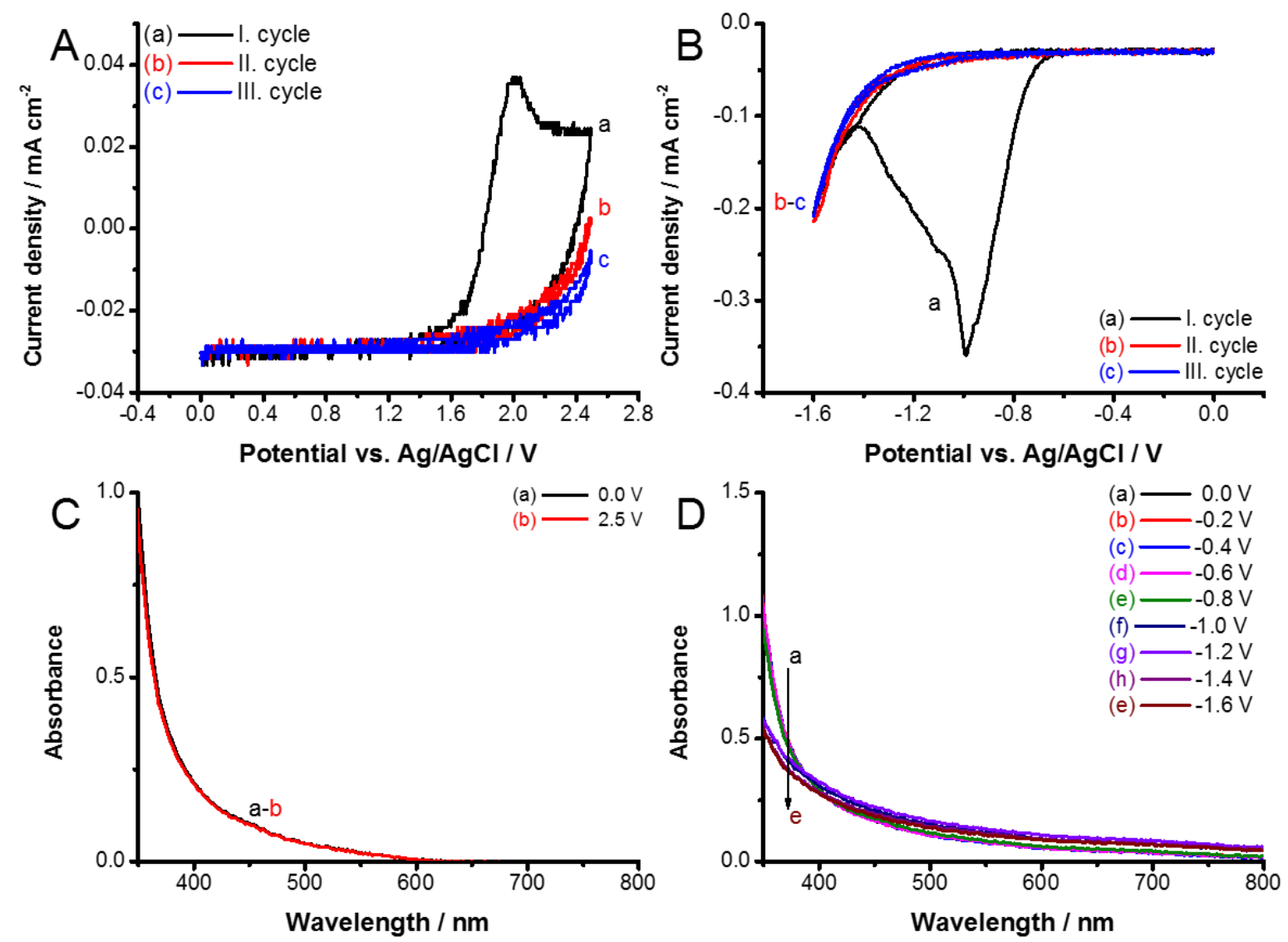

Figure 30. Three subsequent $C V$ curves recorded in $0.1 \mathrm{Bu}_{4} N P F_{6} / D C M$ solution $(10 \mathrm{mV}$ $\mathrm{s}^{-1}$ sweep rate), during the A: oxidation and $\mathrm{B}$ : reduction of the $\mathrm{PbBr}_{2}$ films deposited on $\mathrm{FTO}_{\mathrm{TiO}} \mathrm{O}_{2}$ electrodes. $U V$-vis spectra recorded during $\mathrm{C}$ : oxidation and $\mathrm{D}$ : reduction processes.

\subsubsection{UV-vis Spectroscopic Studies of the Dissolution of $\mathrm{CH}_{3} \mathrm{NH}_{3} \mathrm{PbI}_{3}$ Electrodes}

To see whether the observed behavior is unique to $\mathrm{CsPbr}_{3}$ or it is more general, we extended our study to $\mathrm{MAPbI}_{3}$. Largely, the stability of the films was worse in all electrolytes studied in the present investigation (Figure 31 and Figure 32). The initial absorbance increase (and any opacity), however was not seen in this case. This is a major difference in the behavior of the two perovskite-based electrodes, which may be rooted in the increased sensitivity of iodide based perovskites. Since the stability, obtained in $0.1 \mathrm{M} \mathrm{Bu}_{4} \mathrm{NPF}_{6}$ solution was still not ideal, the electrolyte concentration was decreased to $0.01 \mathrm{M}$ through which a reasonable lifetime was ensured (Figure 31E) 

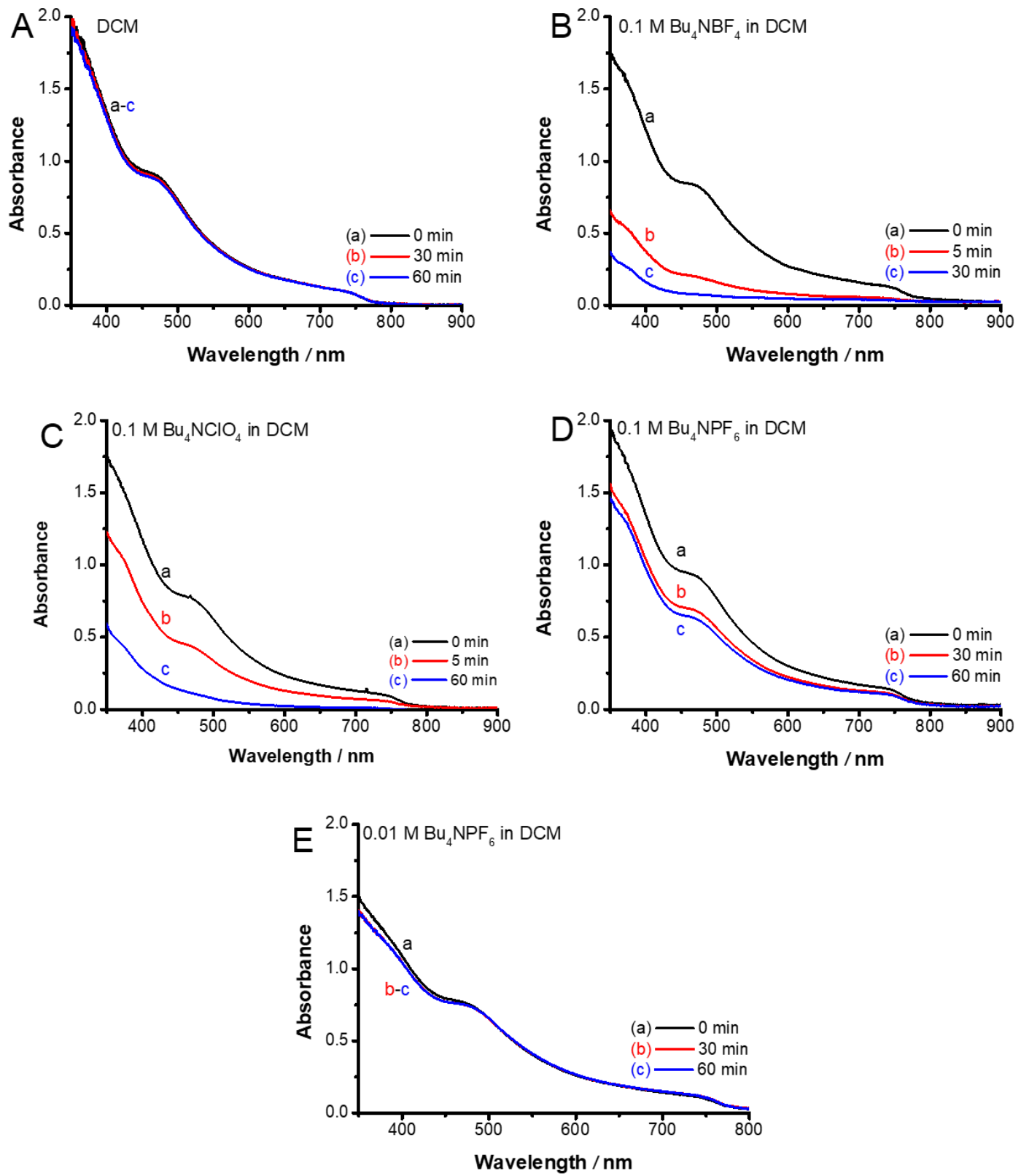

Figure 31. $U V$-vis absorbance spectra of $\mathrm{FTO} / \mathrm{TiO}_{2} / \mathrm{MAPbI}_{3}$ films in different electrolytes, after different immersion times. A: pure DCM, B: $0.1 \mathrm{M} \mathrm{Bu}_{4} \mathrm{NBF}_{4}$ in DCM, C: $0.1 \mathrm{M}$ $\mathrm{Bu}_{4} \mathrm{NClO}_{4}$ in DCM, D: $0.1 \mathrm{M} \mathrm{Bu}_{4} \mathrm{NPF}_{6}$ in DCM, E: 0.01 M BüNPF 6 in DCM.

The normalized absorbance traces, measured at $470 \mathrm{~nm}$ are compared in Figure 32 for the different electrolytes. A similar trend was found for the complexing ability of the used anions as in the case of $\mathrm{CsPbBr}_{3}$ films. 


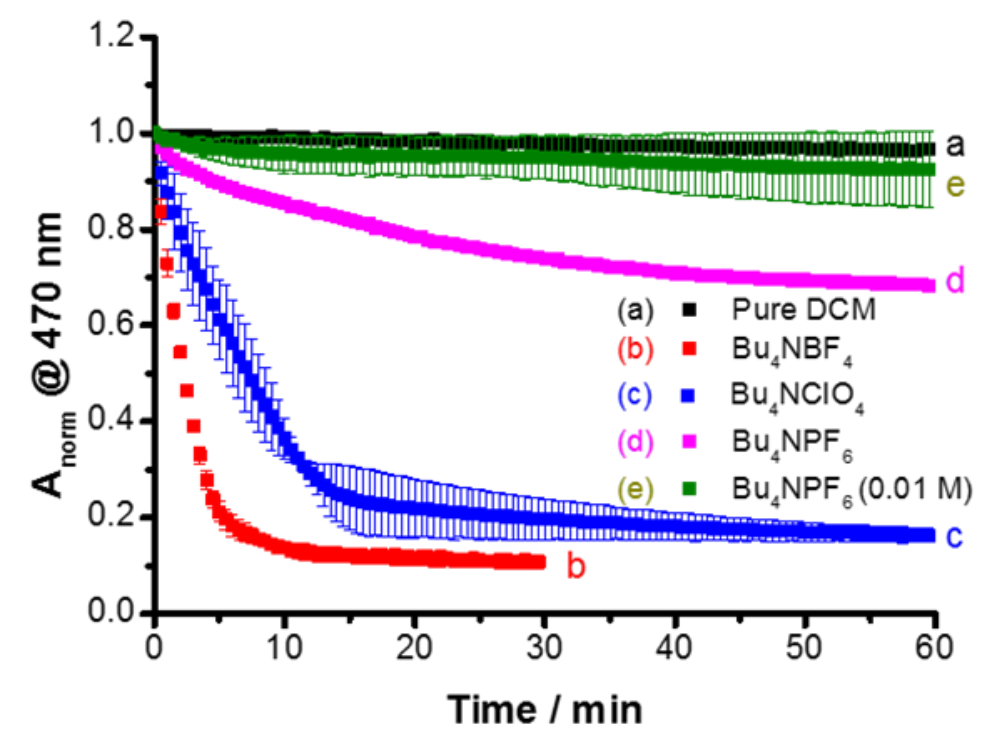

Figure 32. Kinetic curves of the normalized absorbance change at $470 \mathrm{~nm}$, recorded for FTO/TiO $/ \mathrm{MAPbI}_{3}$ in different $\mathrm{DCM}$ based electrolytes. The error bars were derived from the measurement of three separate films.

\subsubsection{Spectroelectrochemistry of $\mathrm{CH}_{3} \mathrm{NH}_{3} \mathrm{PbI}_{3}$ Electrodes}

The observed degradation trend at both positive and negative bias, was the same as in case of the $\mathrm{CsPbr}_{3}$. Spectroelectrochemical studies revealed two oxidation- and one reduction steps, at $E=+0.8 \mathrm{~V},+1.1 \mathrm{~V}$, and $-0.75 \mathrm{~V}$, respectively (Figure 33). Careful inspection of the cathodic wave revealed a shoulder at $E=-0.95 \mathrm{~V}$. These redox events were coupled with a massive decrease in the absorbance related to the dissolution of $\mathrm{MAPbI}_{3}$, and the degradation rate was especially high during reduction (Figure 33B and Figure 34).
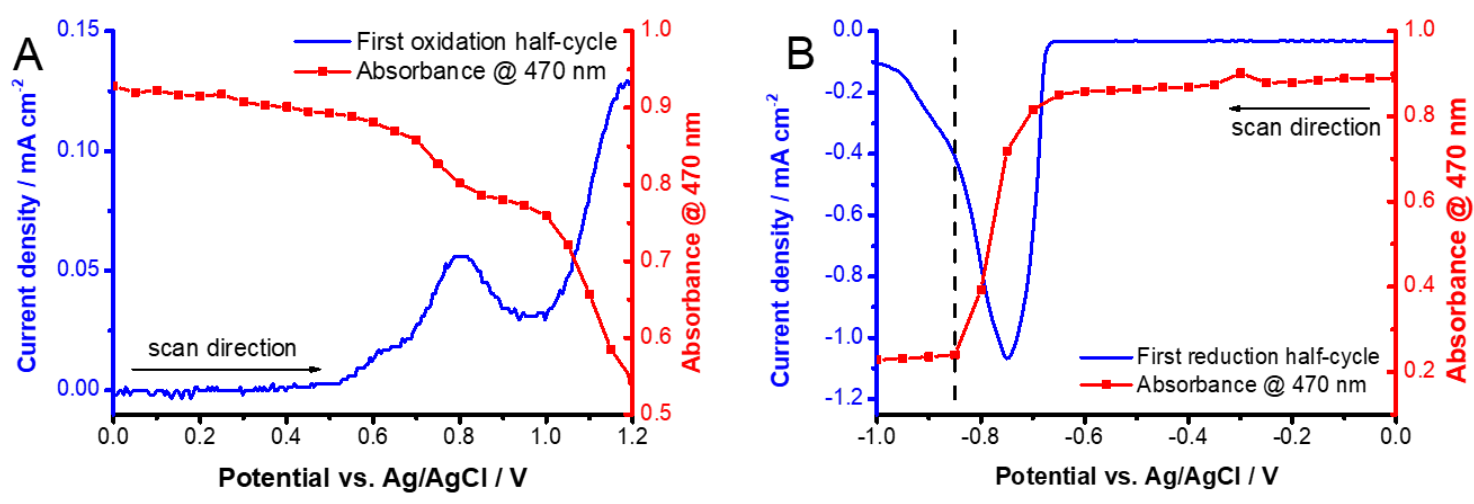

Figure 33. Spectroelectrochemical data, recorded for $\mathrm{FTO} / \mathrm{TiO}_{2} / \mathrm{MAPbI}_{3}$ films in $0.1 \mathrm{M}$ $B u_{4} N P F_{6} / D C M$ solution $\left(10 \mathrm{mV} \mathrm{s}^{-1}\right.$ sweep rate), during the first $\boldsymbol{A}$ : oxidation and $\boldsymbol{B}$ : reduction half cycles together with the absorbance changes at $470 \mathrm{~nm}$. 

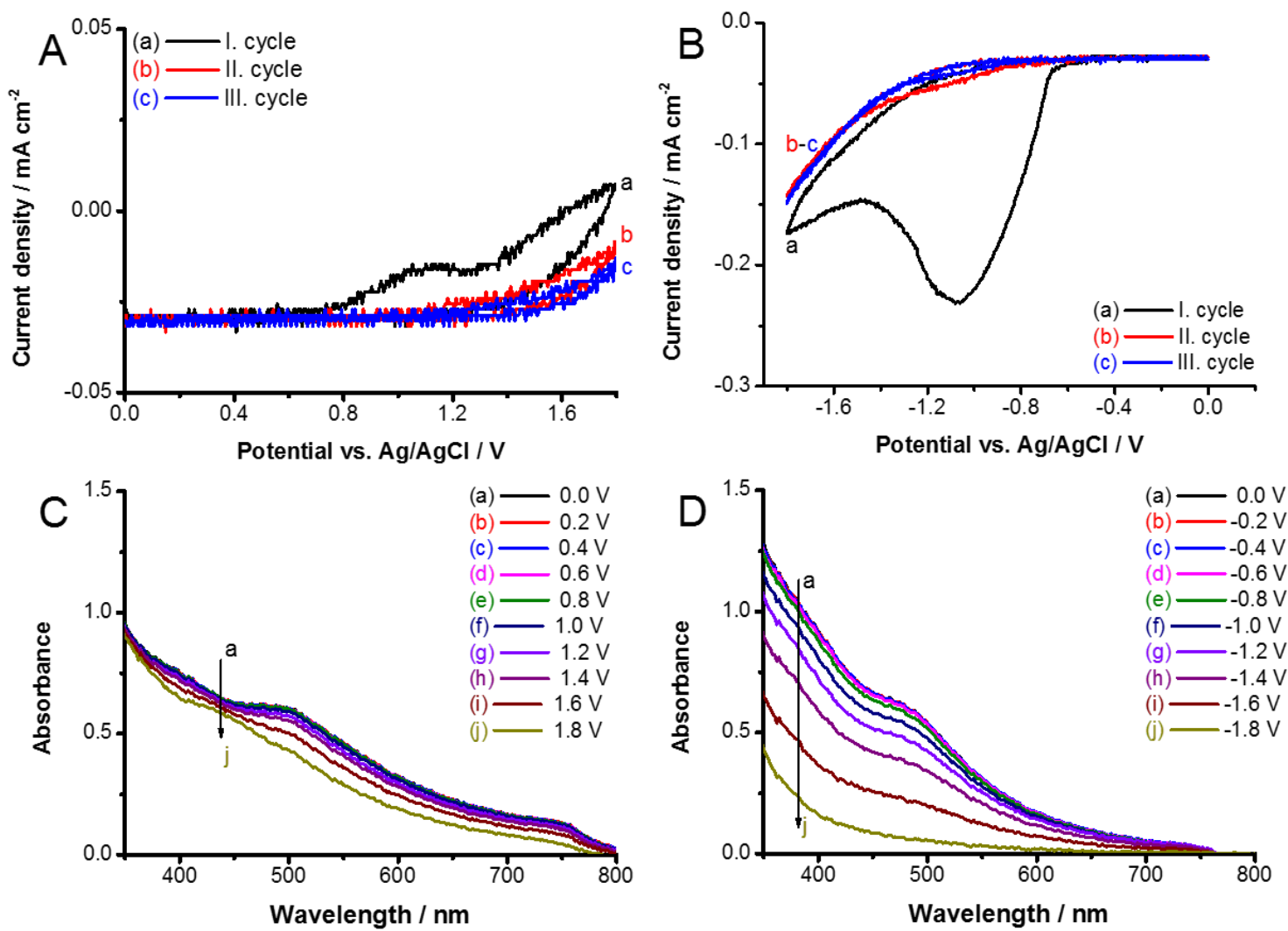

Figure 34. Three subsequent $C V$ curves recorded during the $\boldsymbol{A}$ : oxidation and $\boldsymbol{B}$ : reduction of $\mathrm{FTO} / \mathrm{TiO}_{2} / \mathrm{MAPbI}_{3}$ electrodes in $0.01 \mathrm{Bu}_{4} \mathrm{NPF}_{6} / \mathrm{DCM}$ solution $\left(10 \mathrm{mV} \mathrm{s}^{-1}\right.$ sweep rate).

$U V$-vis spectra recorded during the first $\boldsymbol{C}$ : oxidation and $\boldsymbol{D}$ : reduction half-cycle.

\subsubsection{Spectroelectrochemistry of $\mathrm{PbI}_{2}$ Electrodes}

Similarly to the previous example, control experiments were carried out with the $\mathrm{PbI}_{2}$ films

(Figure 35). Interestingly, $\mathrm{PbI}_{2}$ was less resistant to oxidation compared to $\mathrm{MAPbI}_{3} . \mathrm{Pb}^{2+}$ was reduced at the same potential in case of both films (see Figure 34B and Figure 35B), indicating the similar chemical nature of $\mathrm{Pb}^{2+}$ in the two compounds. 

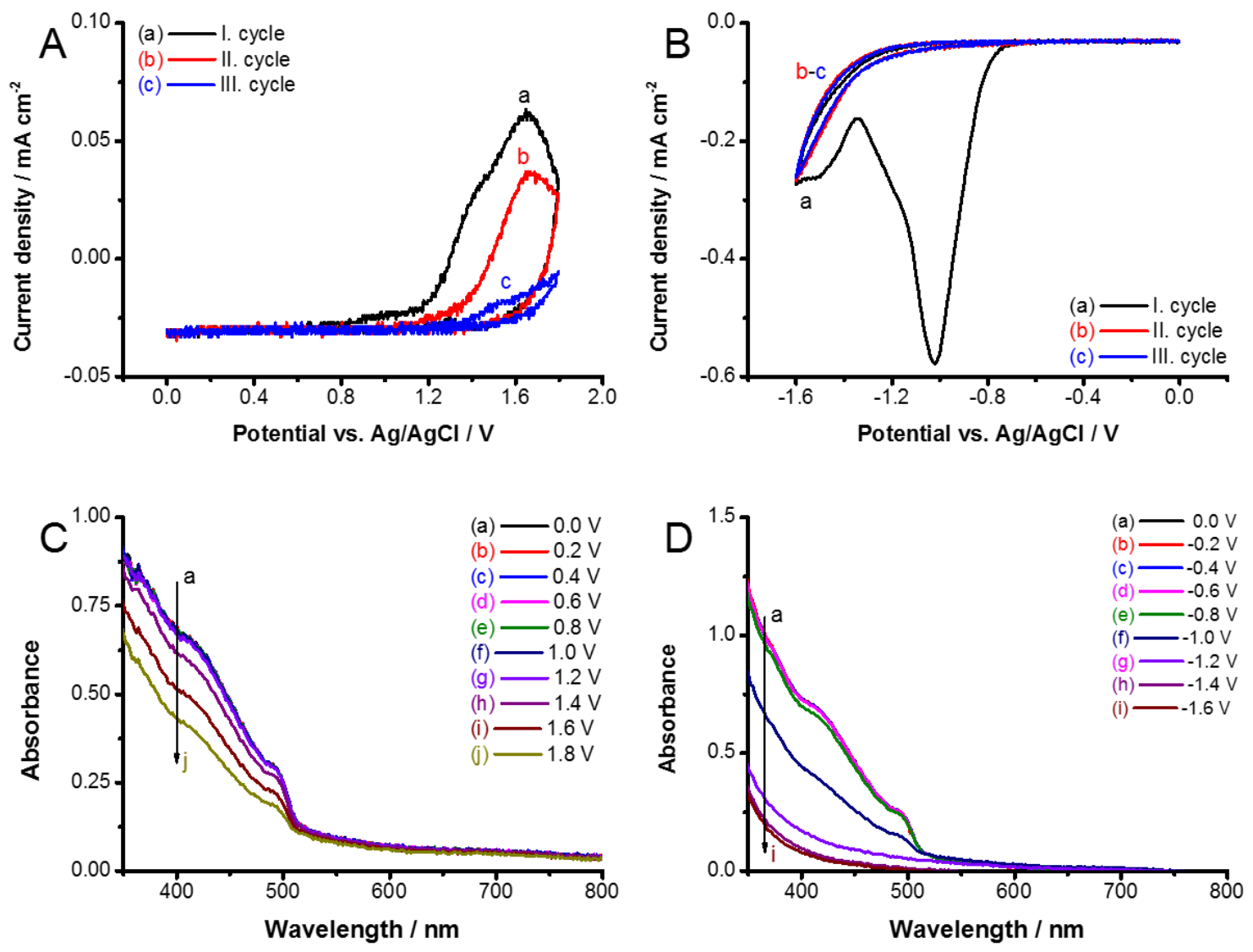

Figure 35. Three subsequent $C V$ curves recorded in $0.1 B u_{4} N P F_{6} / D C M$ solution $(10 \mathrm{mV}$ $s^{-1}$ sweep rate), during the A: oxidation and $\mathrm{B}$ : reduction of the $\mathrm{PbI}_{2}$ films deposited on

$\mathrm{FTO}_{\mathrm{TiO}}$ electrodes. $U V$-vis spectra recorded during the first $\boldsymbol{C}$ : oxidation and $\boldsymbol{D}$ : reduction half-cycles.

\subsubsection{Potential Diagram of Optically Active Perovskite Electrodes}

Taking the above results together, we constructed a potential diagram (Figure 36) to compare the potentials of the various redox events, deduced from the spectroelectrochemical studies. The position of the redox events was always calculated from the onset potential of the redox waves. The band edge positions of the perovskite materials were obtained from previous literature data, and serve semi-quantitative comparison, because the film preparation methods were not identical. ${ }^{[207,208]}$ This representation, together with the previously demonstrated structural analysis, allows to answer the initial question of whether the currents measured during the voltammetric curves are related to charge carrier injection into the materials and/or to real Faradaic events. As for $\mathrm{CsPbr}_{3}$, a good match can be revealed between the potential of the reduction peak and the CB edge; as well as the second oxidation peak and the VB edge. More interestingly, the first oxidation event occurs at a less positive potential than the $\mathrm{VB}$, meaning that the $\mathrm{CsPbr}_{3}$ is irreversibly oxidized before hole injection could take place. Based on all the evidences gathered in this study, these potential 
values should be considered as cathodic and anodic corrosion potentials, respectively. There is a comparable situation in the case of $\mathrm{MAPbI}_{3}$, but here the second reduction peak matches the potential of the $\mathrm{CB}$. This means that an irreversible chemical transformation occurs before the $\mathrm{CB}$ could be populated with electrons. These observations highlight the difference between the solar- and electrochemical cell scenarios because in a solar cell, the photogenerated holes (in the case of $\mathrm{CsPbBr}_{3}$ ) and the electrons (in the case of $\mathrm{MAPbI}_{3}$ ) do not corrode the electrode material as they are transferred quickly to ETL and HTL. If not scavenged quickly, these electrons and holes can induce degradation based on the energy levels illustrated in Figure 36. Addition of an electron acceptor (redox mediator) to the solution can also increase the stability in a similar manner. ${ }^{[154]}$

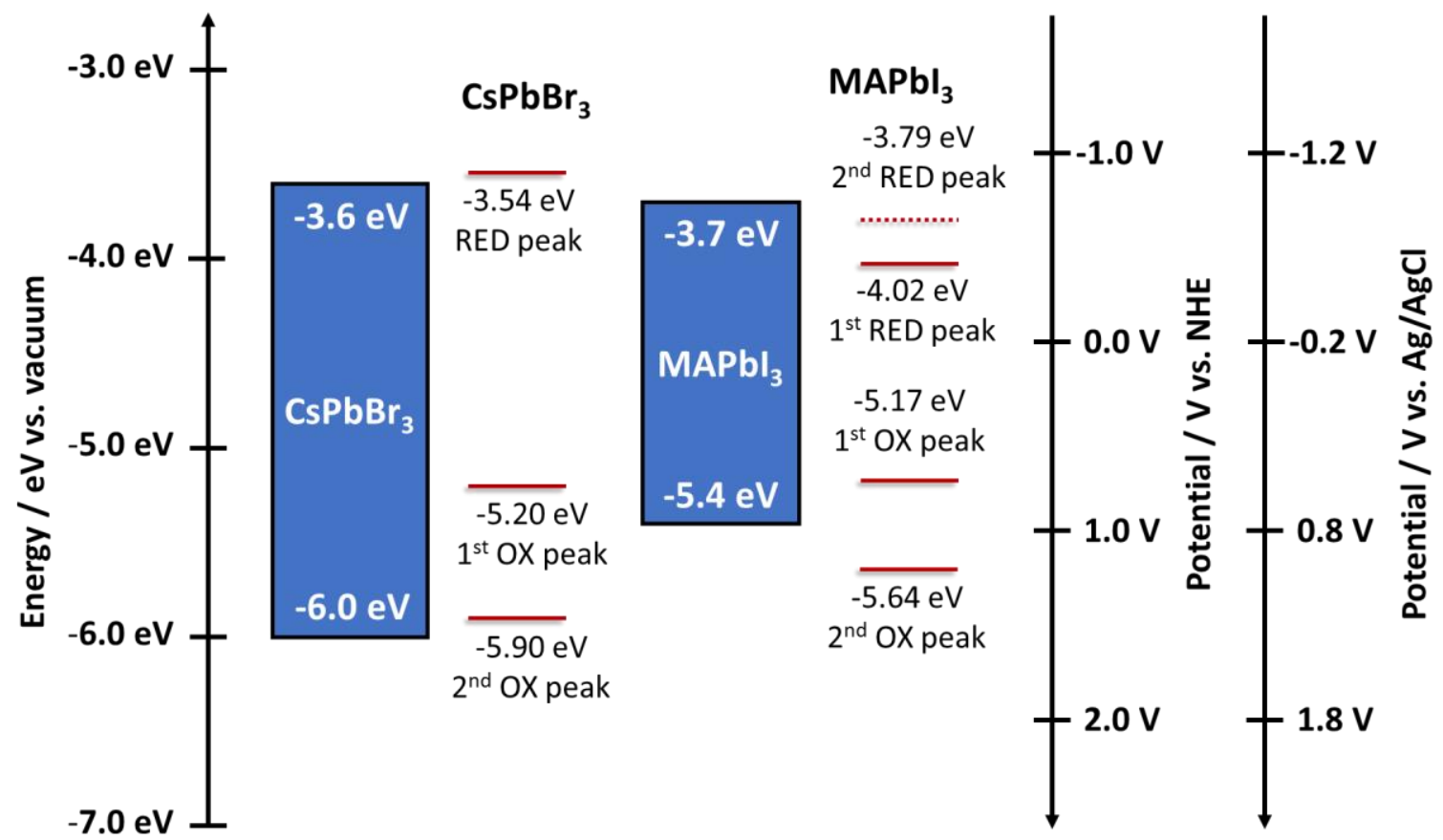

Figure 36. Comparison of the band edge positions and the potential of various redox events detected for the studied two lead halide perovskites.

\subsubsection{Best Practice for Electrochemical Experiments}

To conduct electrochemical studies with metal halide perovskites in a reliable manner, the experiments need to comply with the basic principles of electrochemistry. One needs to make a careful choice of solvent, electrolyte, and electrochemical window to ensure the stability of the electrode within the timeframe of the experiment. Given the sensitivity of perovskite films to polar solvents, one needs to exercise caution while drawing conclusions from the studies performed in aqueous media. ${ }^{[168,170]}$ It should also be noted that conclusions obtained with certain special electrolytes, such as $\mathrm{Li}^{+}$or reversible redox couples ${ }^{[209]}$ which 
are pertinent to certain specific applications, cannot be simply generalized. The researchers should provide data to support the stability test by monitoring the absorption and/or surface spectroscopy measurements before and after electrochemical measurements. This is the only way to ensure that the observed redox processes are chemically reversible. However, there is a safe electrochemical window to conduct electrochemical experiments. One should be able to conduct electrochemical or spectroelectrochemical experiments by adopting the conditions presented in Table 6.

In addition to the experimental considerations, careful analysis of the results is equally important. First of all, the electrochemical process behind the measured redox waves must be clearly identified to draw meaningful conclusions. It should be decided whether the current response reflects a chemical (Faradaic) process or simple charging/discharging (i.e., electron or hole injection). If it is a chemical process, one should study its chemical and electrochemical reversibility. Similarly, it should be clarified whether it is a redox process limited by mass-transport from the solution or a surface confined one. If there is certainly no process other than charge injection, then voltammetric curves can be employed to determine band edge positions. Similarly to organic semiconductors ${ }^{[210]}$, however, the onsets of the redox peaks ${ }^{[154]}$ and not the position of the peak maxima ${ }^{[155]}$ have to be correlated with the respective band edge positions. Finally, during prolonged experiments migration of mobile ions within the perovskite structure might also happen thus complicating the picture even further. ${ }^{[211]}$

Table 6. Parameters to conduct (spectro)electrochemical experiments.

\begin{tabular}{|c|c|c|}
\hline Parameter & $\mathrm{CsPbBr}_{3}$ & $\mathbf{M A P b I}_{3}$ \\
\hline Electrochemical window & $-1.0-+0.6 \mathrm{~V}$ vs $\mathrm{Ag} / \mathrm{AgCl}$ & $-0.65-+0.55 \mathrm{~V}$ vs $\mathrm{Ag} / \mathrm{AgCl}$ \\
\hline Solvent & DCM & $\mathrm{DCM}$ \\
\hline Electrolyte & $\mathrm{Bu}_{4} \mathrm{NPF}_{6}, \leq 0.1 \mathrm{M}$ & $\mathrm{Bu}_{4} \mathrm{NPF}_{6}, \leq 0.01 \mathrm{M}$ \\
\hline Time Window (maximum) & Two hours & One hour \\
\hline Electrode Substrate & $\begin{array}{l}\mathrm{FTO}, \mathrm{FTO} / \mathrm{TiO}_{2} \text {, glassy } \\
\text { carbon }\end{array}$ & $\begin{array}{l}\mathrm{FTO}, \mathrm{FTO} / \mathrm{TiO}_{2} \text {, glassy } \\
\text { carbon }\end{array}$ \\
\hline
\end{tabular}




\subsection{Ultrafast Transient Spectroelectrochemistry of Perovskite Photoelectrodes}

The stability of $\mathrm{CsPbBr}_{3}$ films in $\mathrm{DCM}$ and the $\mathrm{Bu}_{4} \mathrm{NPF}_{6}$ electrolyte has enabled us to conduct electrochemical and spectroelectrochemical measurements on this system. The oxidation and reduction onset were found around +0.65 and $-1.2 \mathrm{~V}$ vs. $\mathrm{Ag} / \mathrm{AgCl}$, respectively for this system (Figure 20C and Figure 22C). The inactivity of $\mathrm{CsPbBr}_{3}$ film between these two onsets offers an electrochemical window to carry out spectroelectrochemical measurements. Thus, a potential window of -0.6 to $+0.6 \mathrm{~V} v s$. $\mathrm{Ag} / \mathrm{AgCl}$ was selected for spectroelectrochemical investigations of $\mathrm{FTO} / \mathrm{CsPbBr}_{3}$ and $\mathrm{FTO} / \mathrm{TiO}_{2} / \mathrm{CsPbBr}_{3}$ electrodes. The absorption spectra recorded before and after the electrochemical cycling within this window showed little changes in the absorption spectra (Figure 37).
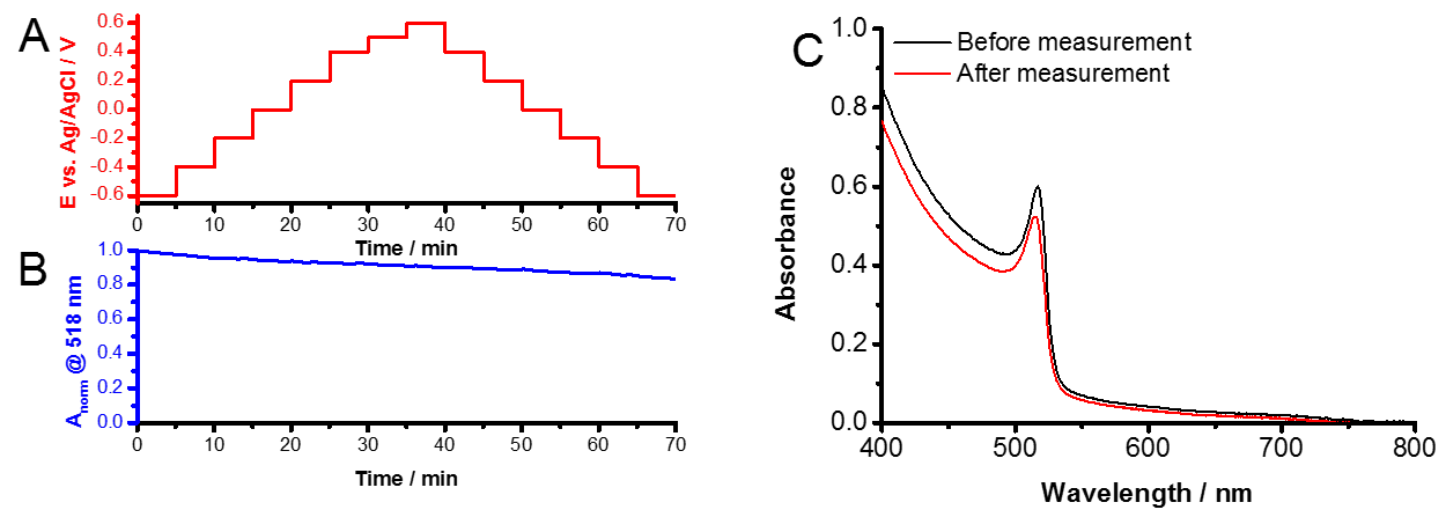

Figure 37. A: Time vs. applied potential curve recorded during electrochemical stability measurements. The potential was ramped from $-0.6 \mathrm{~V}$ to $+0.6 \mathrm{~V}$ and then back to $-0.6 \mathrm{~V}$.

$\boldsymbol{B}$ : Time vs. the normalized absorbance at $518 \mathrm{~nm}$ (excitonic peak) of the $\mathrm{FTO} / \mathrm{TiO}_{2} / \mathrm{Cs} \mathrm{PbBr}_{3}$ film used for the electrochemical stability test. $\mathrm{C}$ : Absorbance spectra of the $\mathrm{FTO} / \mathrm{TiO}_{2} / \mathrm{CsPbr}_{3}$ film before and after the cycling of applied bias.

\subsubsection{Transient Absorption of $\mathrm{CsPbBr}_{3}$ with Applied Bias}

The low exciton binding energy facilitates charge separation following the bandgap excitation of perovskites. The charge separation and charge recombination process in metal halide perovskite films can be readily probed through TAS. ${ }^{[2,93,96,212-215]}$ The time-resolved spectra of a FTO/TiO $2 / \mathrm{CsPbBr}_{3}$ electrode recorded without applied bias is shown in Figure 38. The disappearance of the excitonic band as indicated by the bleaching at $520 \mathrm{~nm}$ represents charge separation. With increasing time, the bleaching at $520 \mathrm{~nm}$ recovers as the photogenerated electrons and holes recombine. ${ }^{[212]}$ 

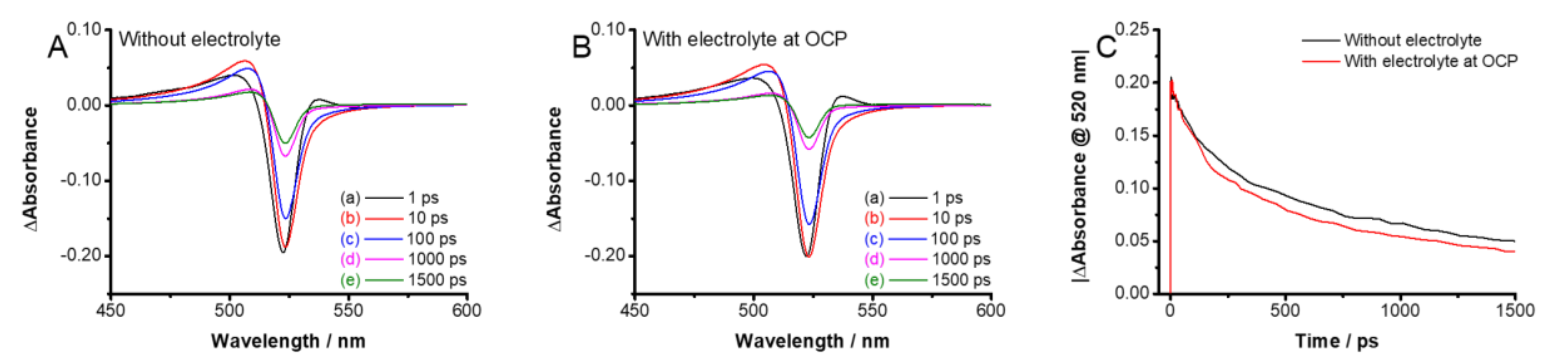

Figure 38. Difference absorption spectra of an $\mathrm{FTO} / \mathrm{TiO}_{2} / \mathrm{Cs} \mathrm{PbBr}_{3}$ film measured $\mathrm{A}$ : in vacuum (without electrolyte) and B: in DCM containing $0.1 \mathrm{MB}_{4} \mathrm{BPF}_{6}$ electrolyte (with electrolyte) under OCP conditions (no external bias was used). C: Respective bleaching recovery profiles recorded in the two different media.

Figure 39 shows the time-resolved TA spectra of $\mathrm{FTO} / \mathrm{TiO}_{2} / \mathrm{CsPbBr}_{3}$ electrodes while being held at four different applied potentials. The significant feature emerging from these spectra is the effect of applied potential on the shape of the spectra during bleaching recovery. The time-resolved spectra recorded at -0.4 and $+0.4 \mathrm{~V}$ show very similar features to those recorded in the absence of applied bias (Figure 39A and B). Thus, qualitatively, the applied bias has no effect on the spectral evolution of transients in this potential regime. On the other hand, the spectra recorded at extreme cathodic $(-1.2 \mathrm{~V})$ and anodic $(+1.0 \mathrm{~V})$ potentials show variation in the spectral features with recovery time (Figure 39C and D). In addition, the maximum bleaching intensity is significantly diminished. Thus, at these extremes a major fraction of the perovskite has degraded and become non-responsive to excitation. These discrepancies in the TAS reaffirm the instability of the perovskite film at these electrochemical bias potentials (in close agreement with the previously shown electrochemical results). Thus, in any electrochemical experiment involving metal halide perovskites one should exercise additional care so that the film is not subjected to irreversible reduction or oxidation. 

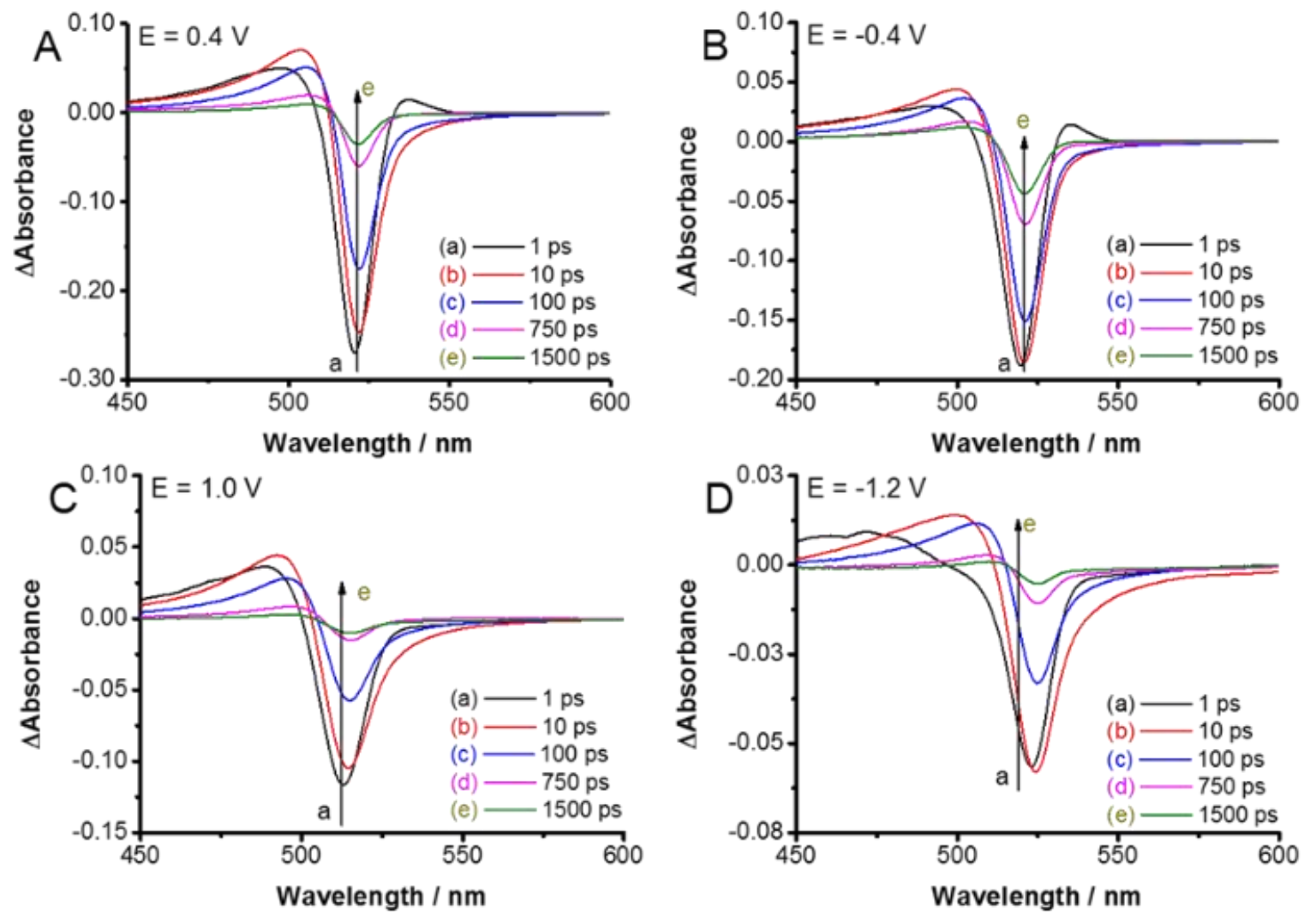

Figure 39. Time resolved transient spectra recorded following $387 \mathrm{~nm}$ laser pulse excitation $\left(4 \mu \mathrm{J} \mathrm{cm}^{-2}\right)$ of $\mathrm{FTO} / \mathrm{TiO}_{2} / \mathrm{CsPbBr}_{3}$ electrode in a spectroelectrochemical cell with deaerated DCM containing $0.1 \mathrm{M} B \mathrm{u}_{4} N P F_{6}$ electrolyte. The spectra were recorded at applied potentials of $\mathbf{A}:+0.4 \mathrm{~V} ; \boldsymbol{B}:-0.4 \mathrm{~V} ; \boldsymbol{C}:+1.0 \mathrm{~V}$ and $\mathrm{D}:-1.2 \mathrm{~V} v \mathrm{~s} . \mathrm{Ag} / \mathrm{AgCl}$.

\subsubsection{Effect of Electrochemical Bias on the Charge Carrier Recombination}

To further probe the effect of electrochemical bias on the charge carrier recombination in $\mathrm{CsPbBr}_{3}$ films the bleaching recovery $(520 \mathrm{~nm})$ was monitored at several different potential values between $-0.6 \mathrm{~V}$ to $+0.5 \mathrm{~V}$ vs. $\mathrm{Ag} / \mathrm{AgCl}$ (Figure 40). As discussed in previous studies, upon laser pulse excitation the bleaching recovery follows simple second order decay. ${ }^{[212]}$ Given the equal concentration of electrons and holes, one expects a linear relationship between reciprocal of concentration of charge carriers (monitored with $\Delta \mathrm{A}$ ) and time. The contribution of trapped charge carriers at high excitation intensity becomes negligible. ${ }^{[216]}$ Also similar magnitude of bleaching was maintained $(\Delta \mathrm{A}=-0.25$ at $520 \mathrm{~nm})$ so that the initial charge carrier concentration is the same for all sets of measurements. The bleaching recovery profiles recorded at different applied potentials and the fitting of the data to second order kinetics (1/ $\Delta \mathrm{A}$ versus time) are shown in Figure 40A and Figure 40B respectively. 

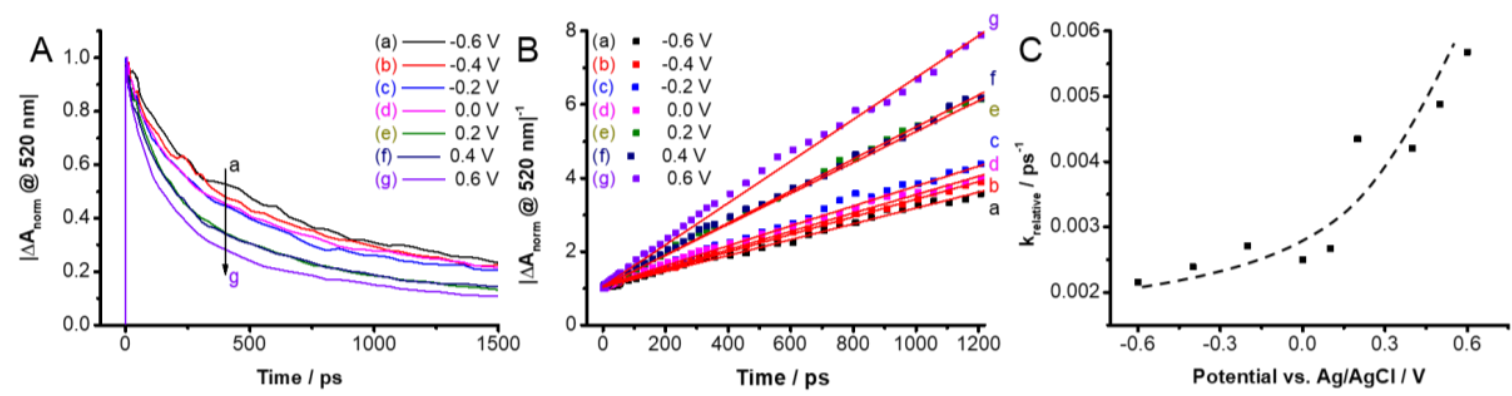

Figure 40. A: Bleaching recovery profiles, $\boldsymbol{B}$ : kinetic fit $1 / \Delta A$ vs. time and $\boldsymbol{C}$ : relative pseudo first order rate constants determined from the slope of plots in $\boldsymbol{B}$ with the dashed line to guide the eye (The magnitude of bleaching was kept constant at $\triangle A=-0.25$ for all these experiments so that initial charge carrier concentration is same in all experiments).

The applied potentials range was between -0.6 and $+0.6 \mathrm{Vvs}$. $\mathrm{Ag} / \mathrm{AgCl}$.

The straight-line plots of $1 / \Delta \mathrm{A}$ versus time confirm the validity of second order kinetics at all applied potentials. Since the initial carrier concentrations were kept similar in all these bleaching recovery experiments, the slopes can be directly compared to obtain relative rate constants or lifetimes of charge carriers. At negative applied potentials, the bleaching recovery exhibits kinetics similar to that recorded in the absence of applied bias. The apparent rate constant $k$ (pseudo first order) obtained from the slope was $\sim 2.5 \cdot 10^{9} \mathrm{~s}^{-1}$ and is not influenced by the applied bias significantly.

The pseudo first order rate constants measured at different applied potentials showed an interesting trend as the applied bias was increased from $-0.6 \mathrm{~V}$ to $+0.6 \mathrm{~V}$ in steps (Figure 40C). At $0.0 \mathrm{~V}$ and more negative potentials the rate constant of charge carrier recombination showed little variation. On the other hand, at anodic bias (more positive than $0.0 \mathrm{~V}$ vs. $\mathrm{Ag} / \mathrm{AgCl}$ ) faster decay kinetics was observed. An increase by a factor of three in the bleaching recovery rate constant was seen as the applied bias was increased to $+0.6 \mathrm{~V}$. Since electrons are depleted from the $\mathrm{TiO}_{2}$ layer under anodic bias, one can expect an additional pathway for photogenerated electrons to participate in the charge injection into $\mathrm{TiO}_{2}$ film $\left(\mathrm{k}_{\mathrm{ET}}\right)$. The competing charge injection process thus renders faster bleaching recovery at anodic bias. These observations are consistent with the mechanism of charge injection from excited molecular sensitizer into $\mathrm{TiO}_{2}$ that is influenced by the applied bias. $^{[217,218]}$

\subsubsection{Reversibility of Bleaching Recovery Kinetics to Applied Bias}

It is imperative to check whether the observed effect of applied electrochemical bias on the bleaching recovery rate constant was reversible. In another, separate experiment the TA spectra were recorded under a forward scan $(-0.6 \mathrm{~V}$ to $+0.5 \mathrm{~V})$ followed by a reverse scan 
$(+0.5 \mathrm{~V}$ to $-0.6 \mathrm{~V})$. The lifetimes derived from the inverse slope of $1 / \Delta \mathrm{A}$ versus time plots are taken as a measure for comparison. These apparent lifetimes obtained at set potentials during forward and reverse scans are presented in Figure 41.

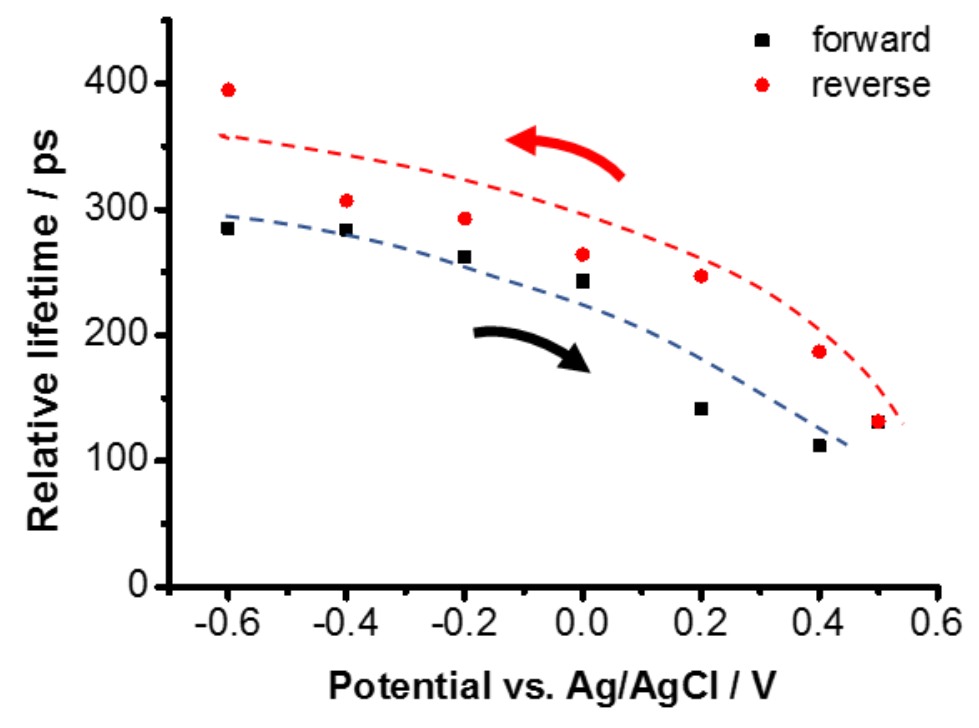

Figure 41. Dependence of bleaching recovery lifetime determined from the inverse slope of the kinetic fit of $1 / \Delta A$ vs. time. Measurements were carried out with $\mathrm{FTO} / \mathrm{TiO}_{2} / \mathrm{CsPbBr}_{3}$ electrodes in deaerated DCM containing $0.1 \mathrm{M} \mathrm{Bu}{ }_{4} N P F_{6}$ electrolyte. The electrodes were biased at set potentials in the range $-0.6 \mathrm{~V}$ to $+0.5 \mathrm{Vvs} . \mathrm{Ag} / \mathrm{AgCl}$ and excited with $387 \mathrm{~nm}$ laser pulse to record time resolved TA spectra.

The decay profiles recorded at a set potential during forward and reverse scans showed the same trend of dependence on the applied bias. Overall, the trend of decreasing lifetimes with increasing positive bias follows the same trend as that seen in Figure 40. There seems to be a small hysteresis in the measured lifetimes when the scan is reversed. Additionally, spectra taken from both the forward and reverse scans show no change in the shape of the features seen in the TA spectra (Figure 42). 

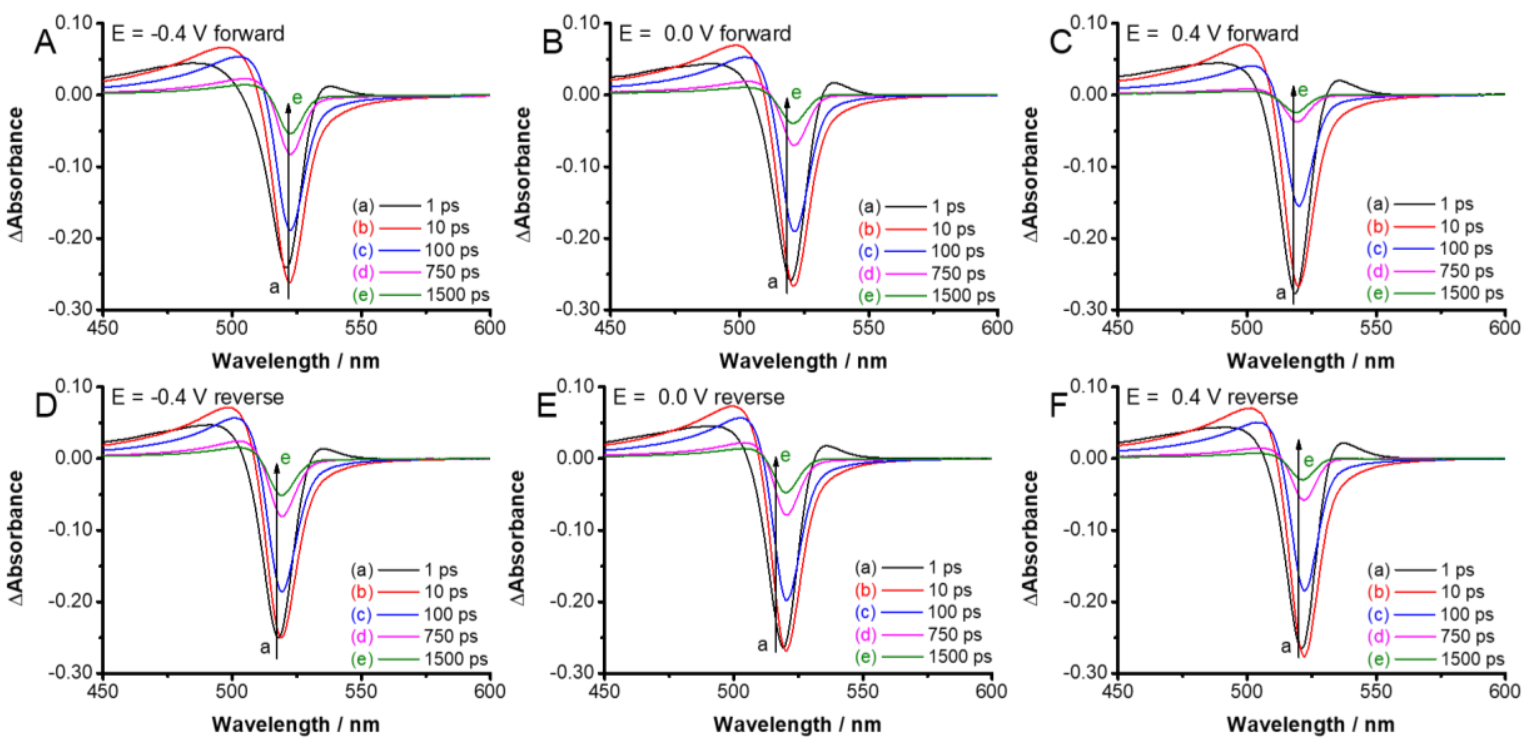

Figure 42. Difference absorption spectra of an $\mathrm{FTO}_{\mathrm{TiO}} / \mathrm{CsPbBr}_{3}$ film at different applied potentials on a forward and reverse scan. Spectra are shown for $A:-0.4 \mathrm{~V}$ forward; B: 0.0 V forward; $\boldsymbol{C}:+0.4 V$ forward; $\boldsymbol{D}:-0.4 V$ reverse $; \boldsymbol{E}: 0.0 \mathrm{~V}$ reverse; $\boldsymbol{F}$ : $+0.4 V$ reverse. The same film was used for all measurements seen here. However, the film was moved to different positions so that degradation due to the pump laser pulse would not occur.

To check the hypothesis that the electron accumulation in $\mathrm{TiO}_{2}$ films increases the lifetime of photogenerated electrons, spectroelectrochemical experiments were carried out with $\mathrm{FTO} / \mathrm{CsPbBr}_{3}$ films (i.e., without $\mathrm{TiO}_{2}$ layer). Interestingly the bleaching recovery was not influenced by the applied electrochemical bias (at least within the studied time-range). The bleaching recovery lifetime remains unchanged at applied potentials in the range of -0.4 $\mathrm{V}-+0.5 \mathrm{~V}$ vs. $\mathrm{Ag} / \mathrm{AgCl}$ (Figure 41). These experimental results further elucidate the role of $\mathrm{TiO}_{2}$ in influencing the charge carrier recombination processes in perovskite films. 


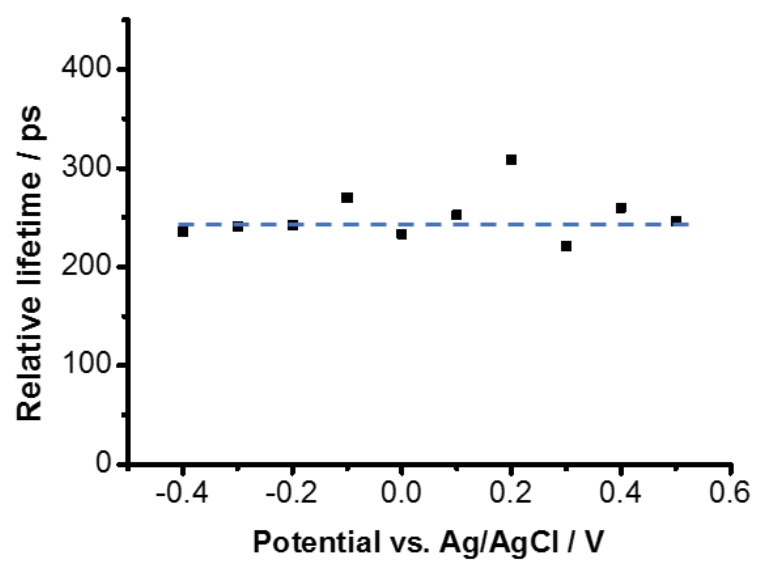

Figure 43. Dependence of bleaching recovery lifetime determined from the inverse slope of the kinetic fit of $1 / \triangle A$ vs. time. Measurements were carried out with $\mathrm{FTO} / \mathrm{CsPbBr} 3$

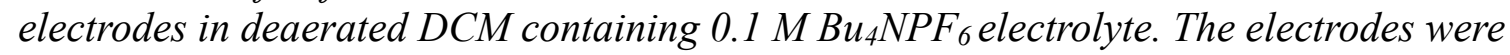
biased at set potentials in the range $-0.4 \mathrm{~V}$ to $+0.5 \mathrm{Vvs} . \mathrm{Ag} / \mathrm{AgCl}$ and excited with $387 \mathrm{~nm}$ laser pulse to record time resolved TA spectra.

\subsubsection{The Influence of $\mathrm{TiO}_{2}$ Layer on Bleaching Recovery Lifetime}

The results presented in Figure 40 and Figure 41 show the decrease in bleaching recovery time as we subject the $\mathrm{FTO} / \mathrm{TiO}_{2} / \mathrm{CsPbBr}_{3}$ electrode to anodic (positive) bias. Since the bleaching recovery is indicative of the charge separation, one can relate the measured lifetime to electron survivability following bandgap excitation of the perovskite film. The band energy diagram in Figure 44 shows a possible scenario of Fermi level changes during open circuit and electrochemical bias conditions. Mesoscopic $\mathrm{TiO}_{2}$ layer, which is in contact with perovskite films, responds to applied bias by accumulating electrons under negative bias or depleting electrons under positive bias. Earlier studies carried out with $\mathrm{TiO}_{2}$ films alone or dye sensitized $\mathrm{SnO}_{2}$ films have shown the electron accumulation under negative bias. ${ }^{[219,220]}$ The electron depletion in $\mathrm{TiO}_{2}$ films under positive bias has pronounced effect on the charge injection rate from excited $\mathrm{Ru}(\mathrm{II})$ polypyridyl complex to metal oxide films. ${ }^{[217,218]} \mathrm{A}$ similar scenario is expected to emerge when a $\mathrm{FTO} / \mathrm{TiO}_{2} / \mathrm{CsPbBr}_{3}$ electrode is subjected to electrochemical bias. If the $\mathrm{TiO}_{2}$ film has excess electrons, as in the case of negative applied bias, we see suppression of electron transfer from excited perovskite to $\mathrm{TiO}_{2}$. Hence charge carrier recombination is the only process dictating the carrier lifetime. On the other hand, under anodic bias, the electron transfer from excited $\mathrm{CsPbBr}_{3}$ to $\mathrm{TiO}_{2}$ is greatly favored and this process now competes with recombination with holes. As a result, the bleaching recovery rate constant increases (or lifetime decreases) as the $\mathrm{FTO} / \mathrm{TiO}_{2} / \mathrm{CsPbBr}_{3}$ electrode is subjected to positive bias. 


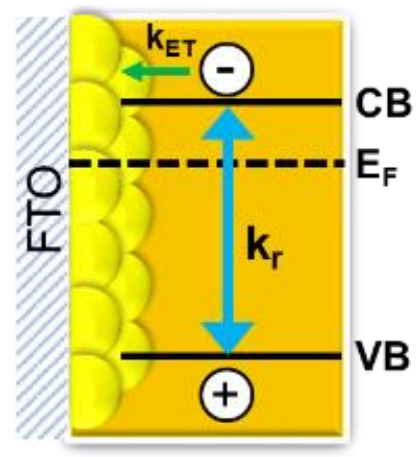

Open Circuit

Potential

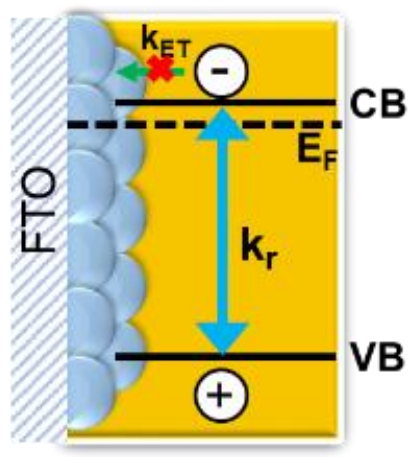

Negative Bias

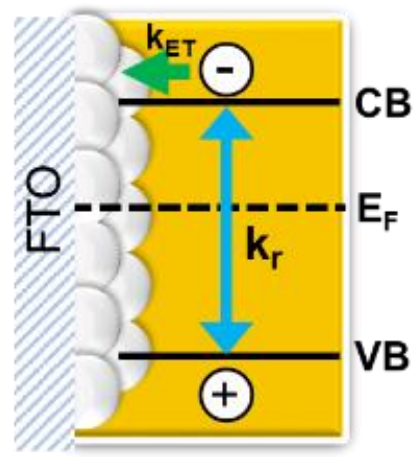

Positive Bias

Figure 44. Schematic diagram illustrating the band energies and the Fermi level response to applied bias. The charge transfer from excited perovskite to $\mathrm{TiO}_{2}\left(k_{E T}\right)$ competes with the charge recombination $\left(k_{r}\right)$ is influenced by the electron accumulation (or depletion) during electrochemical bias

The role of mesoscopic $\mathrm{TiO}_{2}$ film in PSCs remains an intrigue. PSCs can perform well without the presence of a $\mathrm{TiO}_{2}$ mesoscopic layer. For example, planar $\mathrm{MaPbI}_{3}$ based solar cells with a different ETL (e.g., fullerenes) can deliver efficiencies in the range of $20 \% .{ }^{[221,222]}$ Yet the champion cells that utilize mesoscopic metal oxide architecture exhibit superior performance. ${ }^{[223]}$ Thus the role of $\mathrm{TiO}_{2}$ as an ETL that captures electrons from excited $\mathrm{CsPbBr}_{3}$ seems to play an important role in delivering superior device performance. The influence of electron accumulation (or depletion) on the charge recombination rate is a good indication why higher efficiencies are observed in photovoltaic devices containing mesoscopic $\mathrm{TiO}_{2}$ films.

It has been a common practice to manipulate the $\mathrm{j}-\mathrm{V}$ characteristics through light soaking or applying cathode bias. ${ }^{[224]}$ The PSCs show higher efficiency as the cells pretreated with cathodic bias (or light soaking) exhibit higher photovoltage. Under these pretreatment conditions electron accumulation occurs in the $\mathrm{TiO}_{2}$ film as reflected from a small increase in photovoltage. In a previous report on best practices in solar cell characterization, it was demonstrated that an improvement in power conversion efficiency can be observed for a poorly performing solar cell through pretreatment of a negative bias. ${ }^{[41]}$ Indeed the spectroelectrochemical experiments show the effect of cathodic bias on increasing the carrier lifetime. More generally, the in-situ electrochemical TAS measurement can be a useful tool to probe the electron injection process from the excited perovskite film to the ETL, and thus allow a convenient evaluation of different perovskite/ETL pairs. 


\subsection{Light-Induced Phase Segregation in Mixed Halide Perovskite Solar Cells}

\subsubsection{UV-vis Spectroscopic Monitoring of Phase Segregation in $\mathrm{CH}_{3} \mathrm{NH}_{3} \mathrm{PbBr}_{1.5} \mathrm{I}_{1.5}$ Solar Cells}

Two sets of MAPbBr${ }_{1.5} \mathrm{I}_{1.5}$ solar cells were prepared, one with a partial assembly excluding the top gold contact (Figure 12B) and the other complete solar cell assembly (Figure 12A) using the same mixed halide perovskite deposition on a mesoporous $\mathrm{TiO}_{2}$ film. The methylammonium lead halide films were cast using the stoichiometric composition with Br:I ratio of 1:1. The partially assembled cell was used for absorption measurements. The absorption spectra recorded before and after 30 min irradiation with visible light (100 $\mathrm{mW}$ $\mathrm{cm}^{-2}$ ) are shown in Figure 45A. The decrease in absorbance at $620 \mathrm{~nm}$ suggests the disappearance of the mixed halide phase. The concurrent increase in the absorption at shorter and longer wavelengths represents the formation of bromide- and iodide-rich phases. To better visualize this process the difference absorption spectra were recorded (seen in Figure 45B) during the dark recovery. In about three hours, an almost complete recovery of the mixed halide phase can be observed. The exact nature of the absorption changes and recovery can be found in earlier studies on similar thin films. ${ }^{[147,148]}$
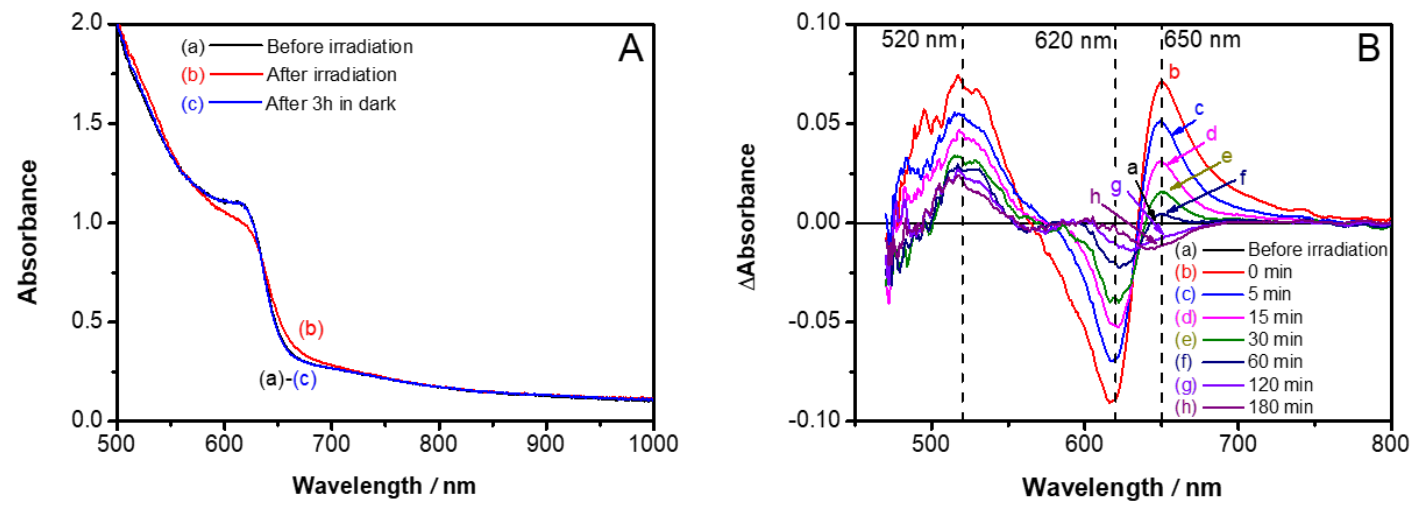

Figure 45. A: Absorption spectra of mixed halide perovskite film (a) before and $(b)$ after irradiation for 30 minutes. Spectrum (c) shows dark recovery after three hours. B:

Difference absorption spectra after 30 min light soaking shows dark recovery. 


\subsubsection{Effect of Phase Segregation on the Efficiency of $\mathrm{CH}_{3} \mathrm{NH}_{3} \mathrm{PbBr}_{1.5} \mathrm{I}_{1.5}$ Solar Cells}

The next step was to evaluate the photovoltaic performance of fully assembled solar cells. The j-V characteristics are shown in Figure 46A. The champion cell that was tested for these measurements yielded a power conversion efficiency of $4.4 \%$, an $\mathrm{V}_{\text {OC }}$ of $1.04 \mathrm{~V}$ and jsC of $6.4 \mathrm{~mA} \mathrm{~cm} \mathrm{~cm}^{-2}$, together with a FF of $66 \%$. The cell was then subjected to visible light irradiation $\left(100 \mathrm{~mW} \mathrm{~cm}^{-2}\right)$ for 30 minutes under open circuit conditions. This allowed us to induce halide ion segregation through visible light excitation (identical to the conditions in Figure 45). Upon evaluation of $\mathrm{j}-\mathrm{V}$ curves of the irradiated cell (Figure 46A), a significant decrease in the solar cell performance was found with $\mathrm{V}_{\text {oc }}$ of $0.91 \mathrm{~V}$ and $\mathrm{J}_{\mathrm{sc}}$ of $4.2 \mathrm{~mA} \mathrm{~cm}^{-2}$ resulting in a net photoconversion efficiency of $1.4 \%$. This phenomenon was also observable for different cells irradiated for 15 and $30 \mathrm{~min}$ (as seen in Figure 46B).
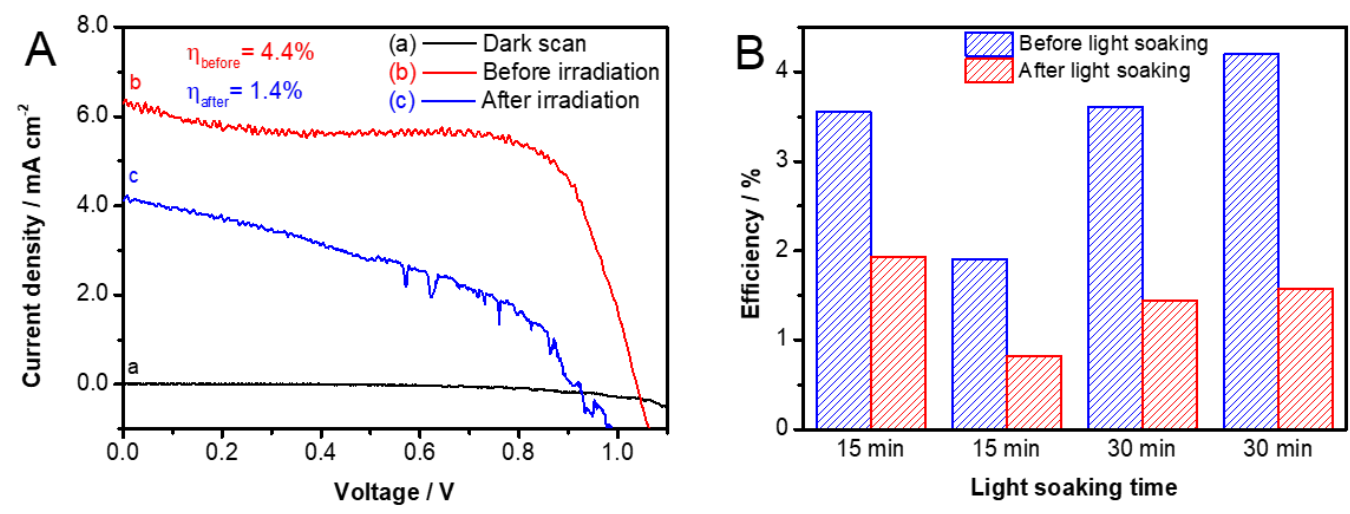

Figure 46. A: $j$-V curves of the champion cell (a) in dark, $(b)$ before light soaking, and (c) after light soaking for 30 minutes. B: Effect of 15 minute and 30 minute light soaking on the performance of four MAPBBr${ }_{1.5} I_{1.5}$ perovskite based cells. The measured photoconversion efficiency before and after light soaking presented in the figure show the effect of light soaking on the photovoltaic performance.

\subsubsection{Effect of Phase Segregation on the Quantum Efficiency of Solar Cells}

We also recorded IPCE data at different times as the cell recovered in the dark (Figure 47A). After inducing phase segregation, a dark current was observed (related to the structural recovery), which distorted the IPCE curves. Employing a $420 \mathrm{~nm}$ filter between the sample and the light source, the dark current could be recorded in the initial regime. Based on these data proper baseline subtraction was applied to all IPCE curves. 

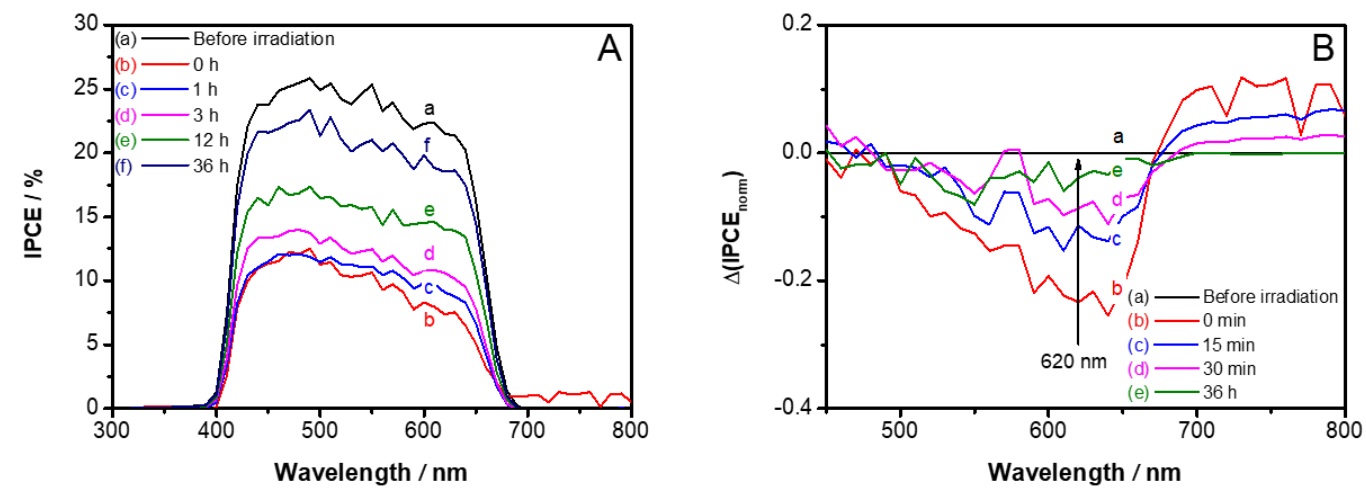

Figure 47. A: IPCE of mixed halide PSC (a) before light soaking and $(b)-(f)$ during dark recovery following $30 \mathrm{~min}$ light soaking. A $420 \mathrm{~nm}$ cut-off filter was used for IPCE measurements and residual dark currents were corrected for IPCE measurements. B: Difference IPCE spectra showing the recovery of the solar cell response in the 500-700 nm region. (The spectra were normalized at $470 \mathrm{~nm}$ and the IPCE recorded before the continuous irradiation was used as the reference.)

The IPCE data recorded immediately after 30 min irradiation shows a nearly $50 \%$ drop in the IPCE values. The IPCE recovered slowly as the cell was left in the dark. Almost $90 \%$ of the recovery was seen in 36 hours. This recovery time is significantly longer than the recovery timescale witnessed in the absorption spectra (Figure 48). Based on this, one can conclude that at the later stages of the recovery process (after 60 minutes from Figure 45B) there is no difference in the light absorption and charge carrier generation processes in the recovering devices. The slower recovery in their electronic properties can be mainly attributed to the hindered charge carrier extraction from the devices. This suggests it takes longer time to alleviate residual traps than the recovery time of the overall absorption.

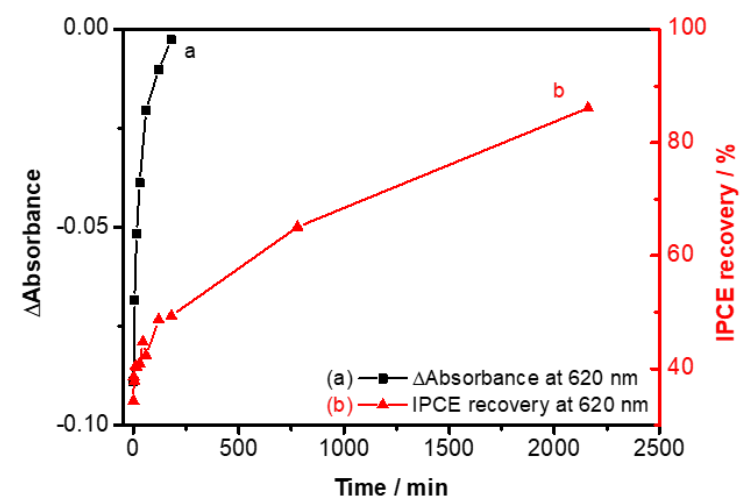

Figure 48. Recovery traces recorded after light soaking of MAPbBr $r_{1.5} I_{1.5}$ based solar cells for 30 minutes (a) the recovery of the bleaching (observed at $620 \mathrm{~nm}$ ) using a partially assembled cell and (b) the overall IPCE recovery of a complete cell. 
Upon closer examination, a change in the shape of the IPCE curves of $30 \mathrm{~min}$ irradiated cell can be seen, compared to the data recoded prior to irradiation. The drop in the IPCE response at longer wavelengths appears to be greater than that at shorter wavelengths. To obtain a closer look at this phenomenon, difference IPCE at different recovery times were recorded following normalization (Figure 47B). The difference IPCE recorded immediately after the $30 \mathrm{~min}$ irradiation of the solar cell shows depletion in the 550-680 nm region with a maximum around $620 \mathrm{~nm}$. This depletion in IPCE matches closely to the decreased absorbance seen in the absorption spectrum after irradiation. This result confirms that the loss of contribution in the IPCE at longer wavelengths arises from the fraction of mixed halide that is lost in the segregation process. As the recovery process in the dark progresses, this depleted IPCE (500-670 $\mathrm{nm}$ ) region recovers. This recovery of IPCE performance parallels the reversibility seen in the photoinduced segregation (see for example Figure 45A and $\mathbf{B}$ ). It is important to note that the irradiated $\mathrm{MAPbI}_{3}$ solar cells do not show such loss of IPCE response in the red region (see Figure 49).

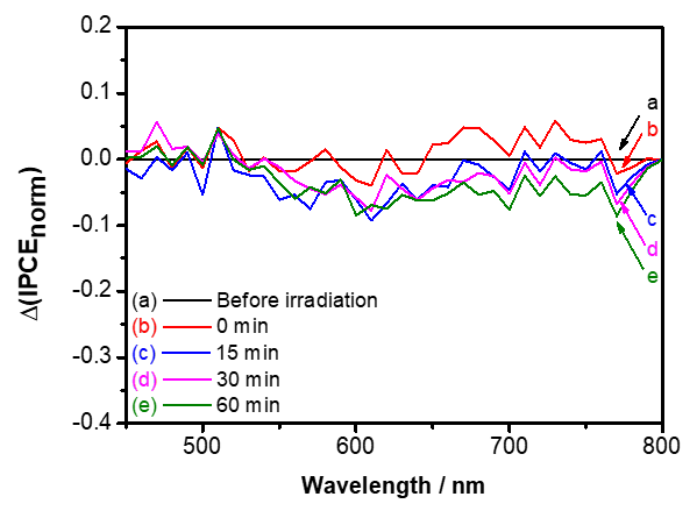

Figure 49. Difference IPCE recorded for $\mathrm{MAPbI}_{3}$ based solar cell irradiated for 30 minutes (the IPCE data were normalized at $450 \mathrm{~nm}$ ).

The observed decrease in IPCE following the $30 \mathrm{~min}$ irradiation of mixed halide film arises from the halide ion segregation and not from the film degradation (see the recovery of the absorption properties of the solar cells). Previous studies showed that low lying I-rich phase serves as a sink for photogenerated electrons and holes. ${ }^{[147]}$ As a result of this accumulation, a prominent emission is seen, arising from the I-rich phase in the photoirradiated films. The gain in the IPCE at wavelengths greater than $700 \mathrm{~nm}$ is rather small and cannot account for the loss we see in the 500-670 $\mathrm{nm}$ region. This discrepancy can be attributed to the halide ion segregated domains within the film which block the flow of charge carriers. While the CBs of mixed halide and segregated phases are isoenergetic, the 
VBs show an energy gradient causing I-rich region to favor the hole accumulation (Figure 50). The I-rich domains thus serve as recombination centers resulting in an eventual decrease in the IPCE and the overall photoconversion efficiency. ${ }^{[78,80,147]}$ As the mixed films recover in the dark, these recombination centers are removed and we see the recovery of photovoltaic performance.
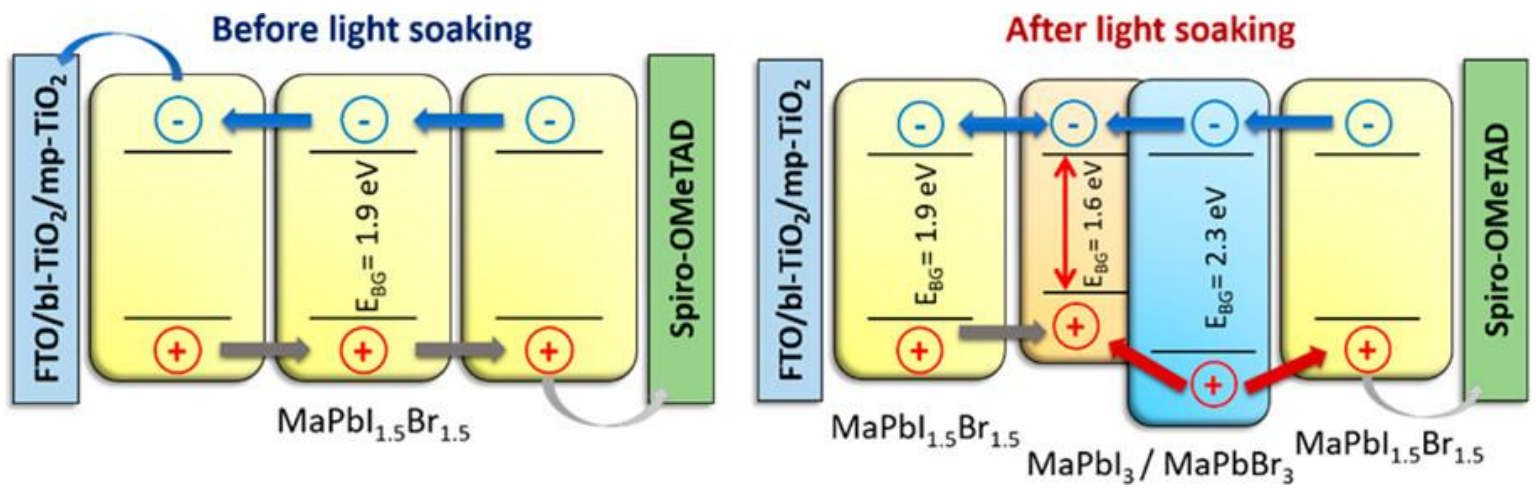

Figure 50. Schematic representation of phase segregation in mixed-halide (MAPbBr $\left.{ }_{1.5} I_{1.5}\right)$ solar cells, and its effect on the charge collection of such devices. 


\subsection{Electrosynthesis of PEDOT Hole Transporter Layer on $\mathrm{CH}_{3} \mathrm{NH}_{3} \mathrm{PbI}_{3}$ Electrodes}

\subsubsection{Spectroelectrochemistry of PEDOT Deposition on $\mathrm{CH}_{3} \mathrm{NH}_{3} \mathrm{PbI}_{3}$ Electrodes}

To monitor the optical changes during electrodeposition, $\mathrm{FTO} / \mathrm{TiO}_{2} / \mathrm{MAPbI}_{3}$ electrodes with a thin $\mathrm{MAPbI}_{3}$ layer were fabricated (Figure 12C). In the previous section we established that in $0.1 \mathrm{M} \mathrm{Bu}_{4} \mathrm{NPF}_{6} / \mathrm{DCM}$ electrolyte, the $\mathrm{MAPbI}_{3}$ layers remain intact (less than $10 \%$ degradation) for around 10 minutes (Figure 32). Note that it was not possible to employ lower electrolyte concentration in this case, because the conductivity of the solution had to be also considered. Furthermore, it had to be ensured that the $\mathrm{MAPbI}_{3}$ layer remains intact during the oxidative electropolymerization. Interestingly, $\mathrm{MAPbI}_{3}$ layers proved to be more resistant to oxidation than reduction (Figure 33). However, EDOT cannot be used as the polymerization precursor, because its polymerization potential would exceed the electrochemical stability window of $\mathrm{MAPbI}_{3}$. To circumvent this issue bis-EDOT was used instead, which in turn allowed the oxidative electropolymerization of PEDOT without degrading the $\mathrm{MAPbI}_{3}$ layer. The polymerization potential can be effectively lowered by using the dimeric unit of a conducting polymer instead of the monomeric unit, caused by the increased conjugation length of the dimer. ${ }^{[225]}$

To determine the electropolymerization potential of bis-EDOT on $\mathrm{FTO} / \mathrm{TiO}_{2} / \mathrm{MAPbI}_{3}$ electrodes, in situ spectroelectrochemical experiments were carried out (Figure 51A-C). The gradual growth of PEDOT was observed during potentiodynamic deposition, indicated by the increasing capacitive current in the range of $0.0 \mathrm{~V}-+0.6 \mathrm{~V}$ (Figure 51A). The peak at $450 \mathrm{~nm}$ on the UV-vis spectrum could be attributed to EDOT oligomers (in the solution) and the broad absorbance between $500-800 \mathrm{~nm}$ to the formation of the PEDOT layer (Figure 51B). To determine the polymerization potential of bis-EDOT on this architecture, the first polymerization half cycle was plotted together with the absorbance increase at $700 \mathrm{~nm}$ in Figure 51C. A sharp rise in the current at $+0.7 \mathrm{~V}$ was observed, which was accompanied by the absorbance increase at $700 \mathrm{~nm}$, confirming the polymerization of bis-EDOT. 

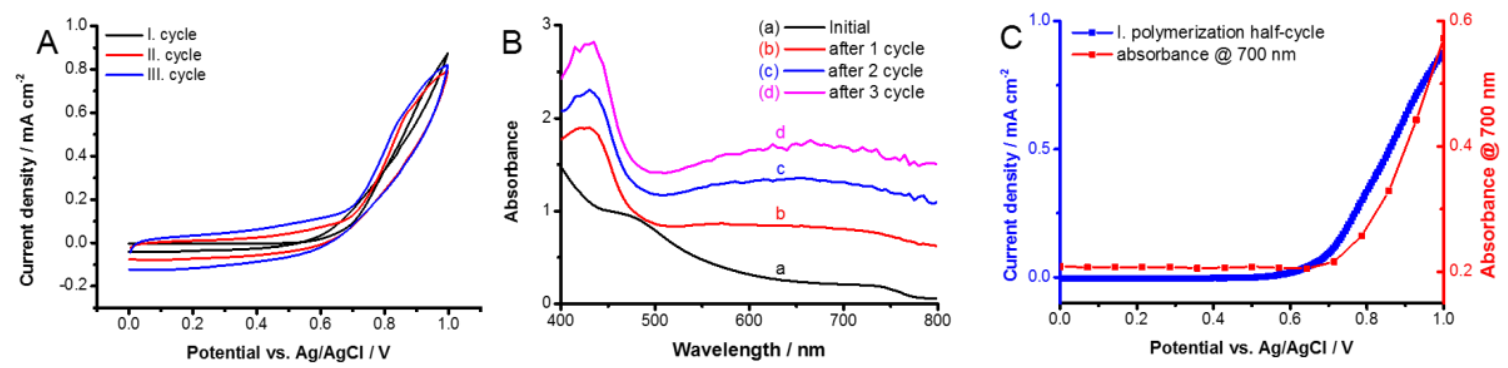

Figure 51. A: Potentiodynamic deposition of PEDOT with $25 \mathrm{mV} \mathrm{s}^{-1}$ sweep rate in a 0.01 $M$ bis-EDOT, 0.1 M Bu ${ }_{4} P_{6} F_{6}$ DCM solution on a solar cell architecture (FTO/bl$\mathrm{TiO}_{2} / \mathrm{mp}-\mathrm{TiO}_{2} / \mathrm{MAPbI}_{3}$ ) employing a thin $\mathrm{MAPbI}_{3}$ layer. $\mathrm{B}: \mathrm{UV}$-vis absorbance spectra recorded after each cycle at $E=0.0 \mathrm{~V}$ during polymerization. $C$ : First half-cycle of the potentiodynamic deposition plotted together with the absorbance change at $700 \mathrm{~nm}$.

Subsequently, our focus shifted to solar cell architectures employing $\mathrm{MAPbI}_{3}$ with regular thickness (Figure 12C). First, the effect of the applied potential on the polymerization rate was studied. Although polymerization starts already at $+0.7 \mathrm{~V}$, it was rather slow, and prolonged exposure to the electrolyte should be avoided. As seen in Figure 52A adequate polymerization rate could only be attained above $+0.9 \mathrm{~V}$. In addition, by varying the polymerization charge density, the thickness of the formed PEDOT layer can be fine-tuned, as deduced from the absorbance spectra (Figure 52B-C). It is apparent that this steady growth is a general feature of all applied potentials as seen in Figure 52C. Furthermore, the UV-vis absorption features of the original $\mathrm{MAPbI}_{3}$ were conserved in all cases, thus no major degradation occurred.
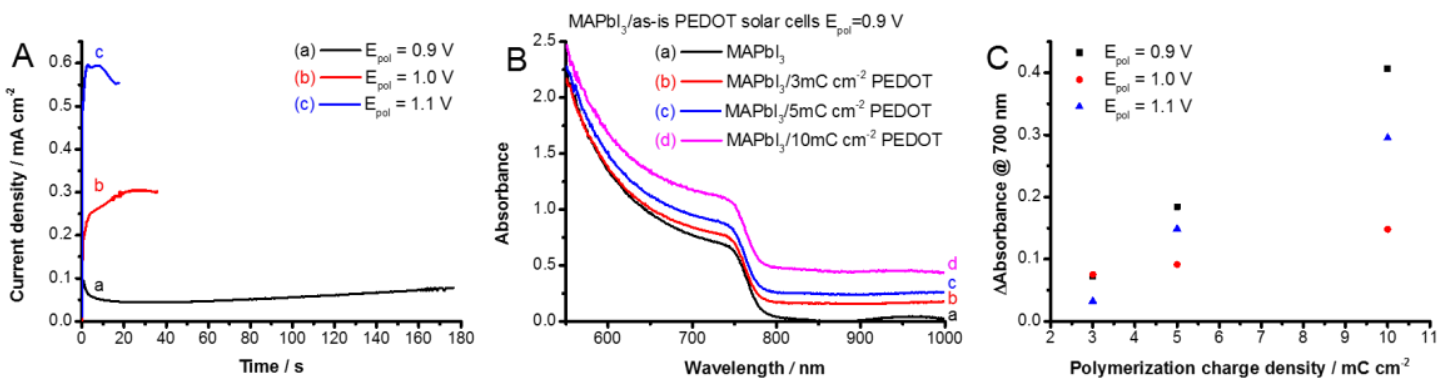

Figure 52. A: Potentiostatic deposition of PEDOT at different potentials in a $0.01 \mathrm{M}$ bisEDOT, $0.1 \mathrm{MB} \mathrm{Bu}_{4} \mathrm{NPF} \mathrm{F}_{6} \mathrm{DCM}$ solution on a solar cell architecture employing regular thickness $\mathrm{MAPbI}_{3}$ layers with a polymerization charge density of $10 \mathrm{mC} \mathrm{cm}^{-2} . \mathbf{B}: U V$-vis absorbance spectra of solar cells after PEDOT electrodeposition at $E=+0.9 \mathrm{~V}$ for different polymerization charge densities. $C$ : The absorbance change of the solar cells compared to a HTM-free cell by varying the polymerization charge density at different applied potentials. 


\subsubsection{Morphological Features of Electrodeposited PEDOT}

To reveal the morphological features of the deposited PEDOT layers, SEM images were captured for a solar cell, where PEDOT was deposited at $\mathrm{E}=+1.0 \mathrm{~V}$ with $\mathrm{Q}_{\mathrm{pol}}=5 \mathrm{mC} \mathrm{cm} \mathrm{m}^{-2}$. The top-view images show that the formed PEDOT completely covers the $\mathrm{MAPbI}_{3}$ layer (Figure 53A). The smooth $\mathrm{MAPbI}_{3}$ surface was not visible anymore, instead a furry polymer coating developed. To determine the thickness of the formed PEDOT, cross-sectional FIBSEM images were taken (Figure 53B). These further confirmed the homogeneity of the PEDOT layer and that the $\mathrm{MAPbI}_{3}$ remains intact during the electrodeposition. From these images the actual PEDOT thickness was determined and compared with the theoretical value calculated from the polymerization charge density (Figure 53C). These values fall in the range of the HTM thicknesses employed in the case of $\mathrm{MAPbI}_{3}$ solar cells using PEDOT. ${ }^{[194,195]}$ Raman-spectroscopic studies (Figure 53D) further confirmed the presence of a PEDOT layer on the surface of the PSCs. ${ }^{[226]}$
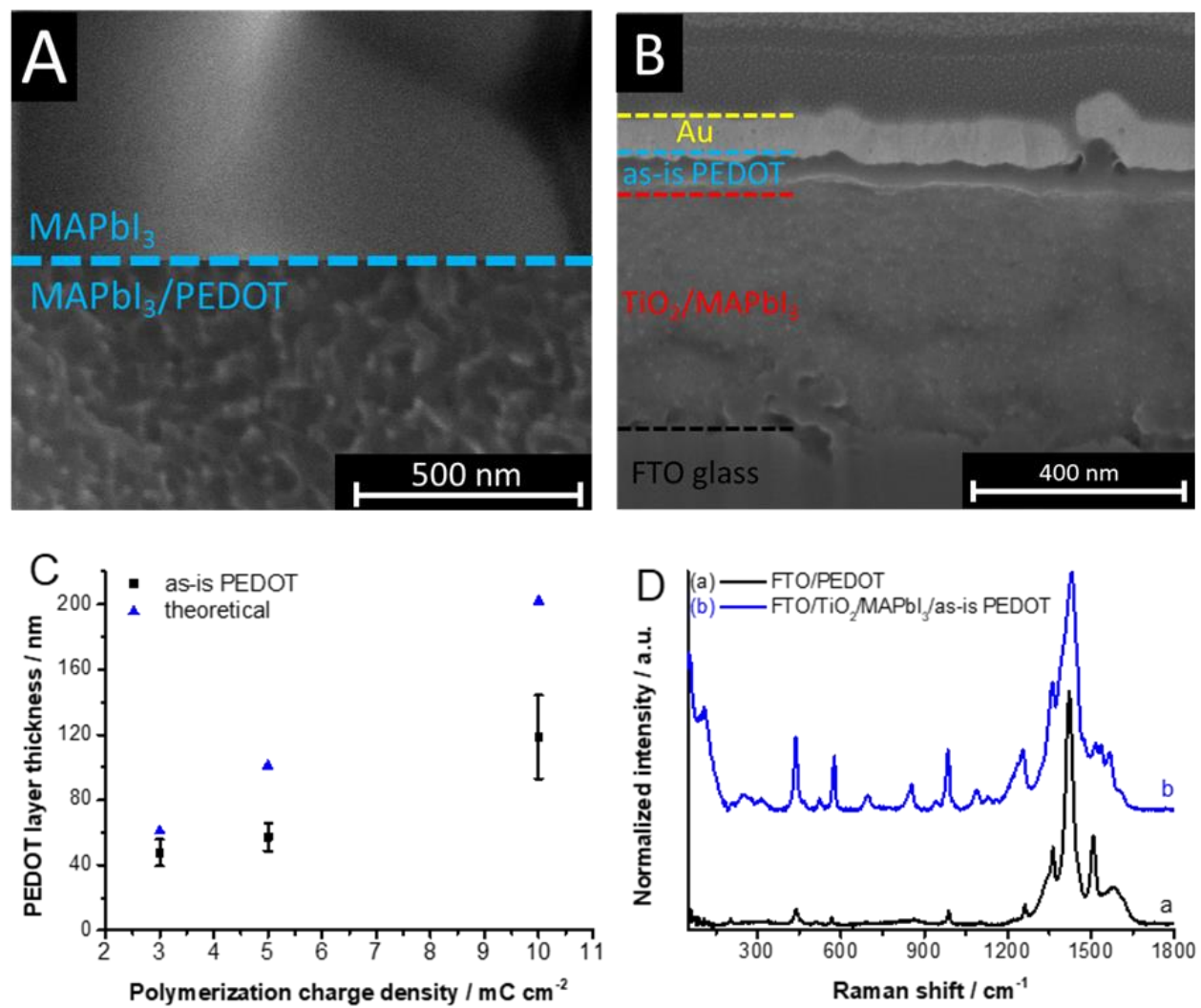

Figure 53. A: Top-view SEM image before (upper part) and after PEDOT (lower part) electrodeposition. B: Cross sectional FIB-SEM image of a solar cell, where the PEDOT electrodeposition was carried out at $E=+1.0 \mathrm{~V}$ with $Q_{p o l}=5 \mathrm{mC} \mathrm{cm}^{-2} . C$ : Theoretical and actual PEDOT layer thicknesses (determined from cross-sectional SEM images). D: Raman-spectra of a fully assembled solar cell architecture and a FTO/PEDOT reference. 


\subsubsection{Efficiency of Solar Cells Employing Electrodeposited PEDOT Hole Transporter}

Subsequently, we evaluated the performance of the solar cells employing the electrochemically deposited PEDOT hole-transporting layers without any post-treatment. The different parameters extracted from the j-V curves are summarized in Figure 54. The cells employing as-is PEDOT performed poorly (i.e., conversion efficiency below 2\%). A general observation was that all cells suffered from low $\mathrm{V}_{\mathrm{OC}}$ and FF, which is indicative of excessive recombination in the solar cells (Figure 54).
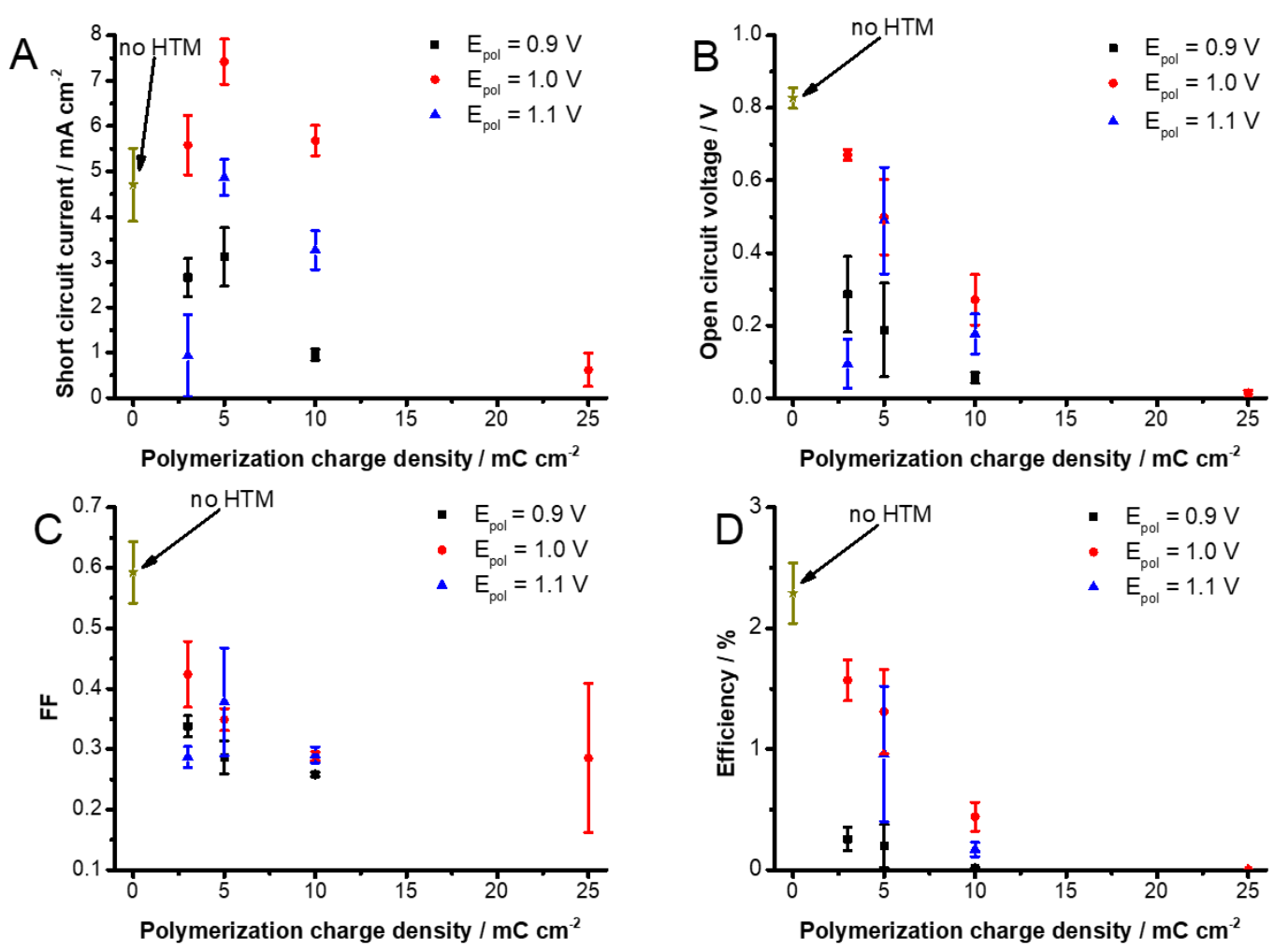

Figure 54. The effect of PEDOT thickness (polymerization charge density) in a FTO/TiO $/ \mathrm{MAPbI}_{3} / \mathrm{PEDOT}$ solar cell on the A: short circuit current density, B: open circuit voltage, $\boldsymbol{C}$ : fill factor, and $\boldsymbol{D}$ : overall efficiency. The solar cells were tested under 1 sun $\left(100 \mathrm{~mW} \mathrm{~cm}^{-2}\right)$ illumination, with a $50 \mathrm{mV} \mathrm{s}^{-1}$ sweep rate.

To measure the $\mathrm{V}_{\mathrm{OC}}$ of the devices more precisely and to see the stability of the $\mathrm{V}_{\mathrm{OC}}$ the devices were subjected to periodic $30 \mathrm{~s}$ of dark-bright cycles under open circuit conditions (Figure 55A). These measurements yielded the same trend as the $\mathrm{j}-\mathrm{V}$ curves, furthermore they revealed that the $\mathrm{V}_{\mathrm{OC}}$ of the devices was stable for a prolonged time. The dark $\mathrm{j}-\mathrm{V}$ characteristics of the solar cells further reinforced the notion that excessive recombination is behind the low performance of the fabricated solar cells (Figure 55B). As the thickness of the electrodeposited PEDOT increased the measured VOC decreased and the 
magnitude of the dark current increased. This indicated that a too thick PEDOT layer (> 150 $\mathrm{nm}$ ) can deleteriously affect the performance of PSCs. This may be an indicator that excessive recombination takes place in the as-is PEDOT layer, and by increasing the HTL thickness more charge carriers are lost by this recombination. A valid strategy to overcome this issue is to utilize different post-treatments, to enhance the properties of the deposited PEDOT layers.
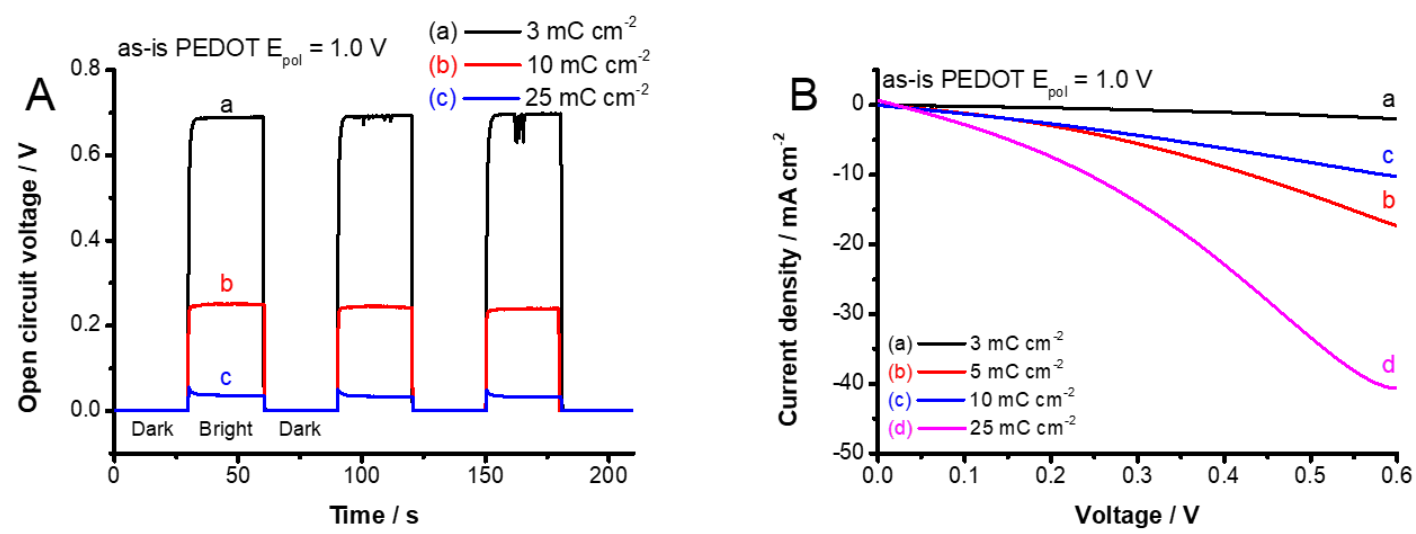

Figure 55. The effect of PEDOT thickness (polymerization charge density) in a $\mathrm{FTO} / \mathrm{TiO}_{2} / \mathrm{MAPbI}_{3} / \mathrm{PEDOT}$ solar cell for PEDOT layers electrodeposited at $E=+1.0 \mathrm{~V}$. $A$ : on the open circuit voltage $\boldsymbol{B}:$ on the $j$-V profile of the solar cell in the dark $\left(50 \mathrm{mV} \mathrm{s} \mathrm{s}^{-1}\right)$.

\subsubsection{Electrochemical Post Treatment of the Deposited PEDOT Hole Transporter}

The solar cell tests indicate that the conductivity of the PEDOT is either insufficient or too high. To overcome the issue, we manipulated the doping level, and thus the charge carrier concentration of PEDOT, via a simple electrochemical conditioning step (Figure 56). 


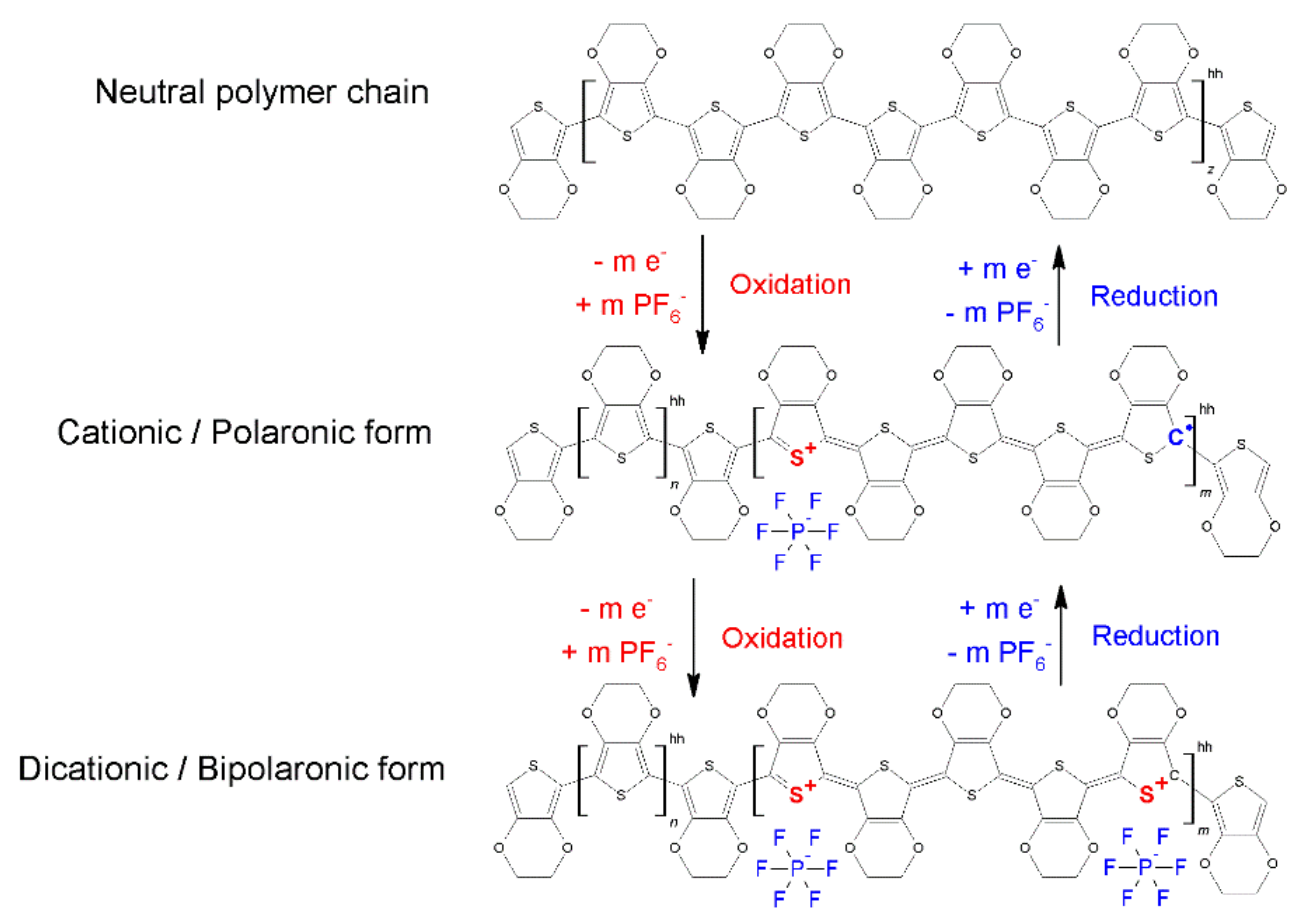

Figure 56. Schematic representation of PEDOT doping.

From the previous experiments, PEDOT films deposited at $+1.0 \mathrm{~V}$, with a $\mathrm{Q}_{\mathrm{pol}}=3$ $10 \mathrm{mC} \mathrm{cm}^{-2}$ seemed to be optimal to obtain reasonable currents. Such PEDOT layers were subjected to either oxidation at $+0.6 \mathrm{~V}$ or reduction at $-0.6 \mathrm{~V}$ for $\mathrm{t}=60 \mathrm{~s}$ (Figure 57A and Figure 57B).
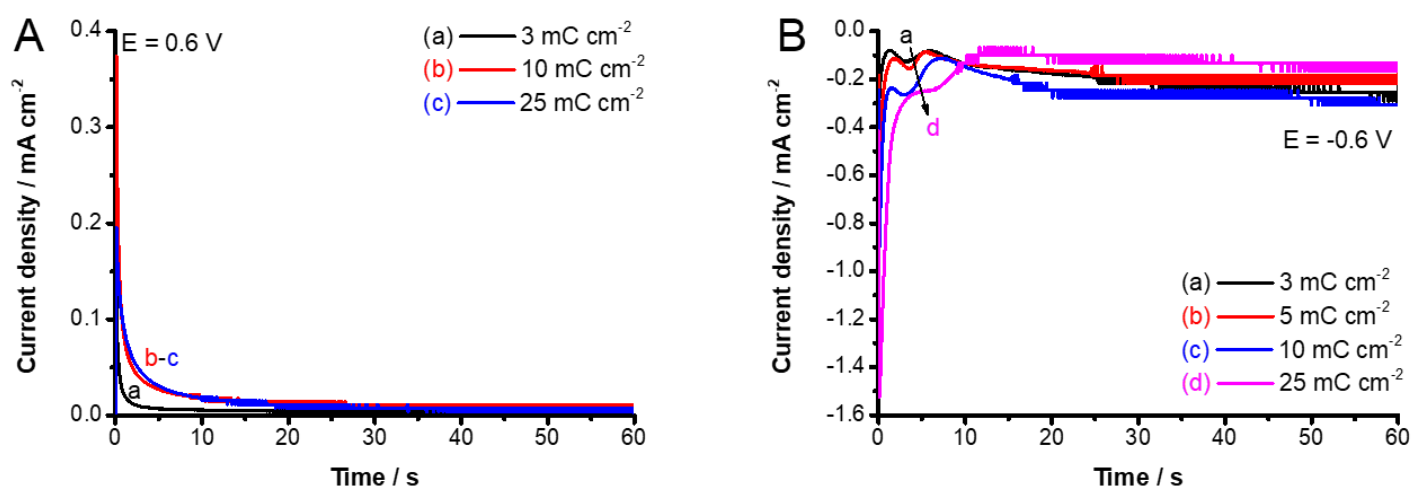

Figure 57. Chronoamperometric curves recorded for the post-treatment of a $\mathrm{FTO} / \mathrm{TiO}_{2} / \mathrm{MAPbI}_{3} / \mathrm{PEDOT}$ solar cell, where the PEDOT layers were electrodeposited at $\mathrm{E}$ $=+1.0 \mathrm{~V}, \boldsymbol{A}:$ when an oxidative post-treatment at $E=+0.6 \mathrm{~V}$ and $\boldsymbol{B}:$ when a reductive post-treatment at $E=-0.6 \mathrm{~V}$ was employed.

No redox-event occurred during oxidation (Figure 57A), which is not surprising since electropolymerization inherently results in PEDOT films in their fully oxidized state. Consequently, this treatment had negligible effect on the $\mathrm{V}_{\mathrm{OC}}$ of the prepared devices 
(Figure 58). The picture is much murkier in the case of reduction (Figure 57B). There are two distinct redox events that both depend on the deposited PEDOT layer thickness. The first (between 0 and $10 \mathrm{~s}$ ) is associated with the reduction of PEDOT, while the second (between 20 and $60 \mathrm{~s}$ ) is related to the electroreduction of the $\mathrm{MAPbI}_{3}$ layer beneath the PEDOT film. Interestingly, this reduction step greatly improved the $\mathrm{V}_{\mathrm{OC}}$ of the solar cells (Figure 58), even when the partial destruction of the $\mathrm{MAPbI}_{3}$ layer occurred. This trend is not unprecedented, as it was proposed that reducing the doping level of PEDOT improves the performance of PSCs ${ }^{[194]}$.

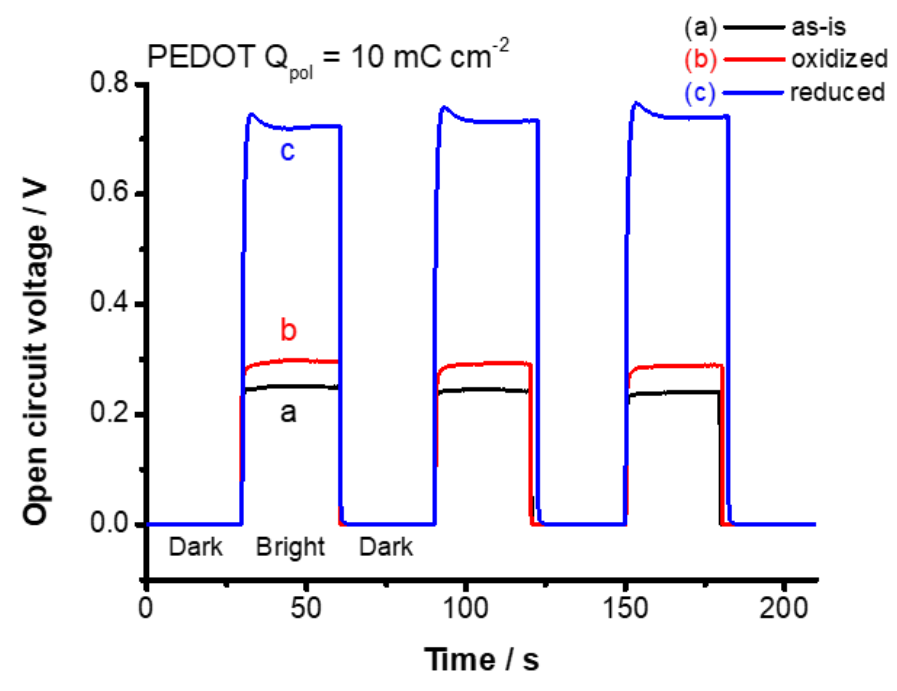

Figure 58. Development of the open circuit voltage in a FTO/TiO $/ \mathrm{MAPbI}_{3} / \mathrm{PEDOT}$ solar cell for the PEDOT layers electrodeposited at $E=+1.0 \mathrm{~V} \mathrm{for} Q_{p o l}=10 \mathrm{mC} \mathrm{cm}^{-2}$ as a function of redox treatment.

This reductive post-treatment method, however, had an unintended side-effect, as revealed by cross sectional SEM images (Figure 59A-B). While the reduction of PEDOT is beneficial for its performance, the post-treatment destroys some of the $\mathrm{MAPbI}_{3}$ from the underlying layer. Excessive void formation can be observed even in the case of thicker PEDOT coatings (greater polymerization charge density). These voids disrupt the inner structure of the perovskite layer, which again affect the device performance in a deleterious manner. Interestingly, the PEDOT layer remains completely intact on the surface of the $\mathrm{MAPbI}_{3}$ layer. However, this the PEDOT coating is not capable of protecting the perovskite layer from the reductive electrochemical treatment. 

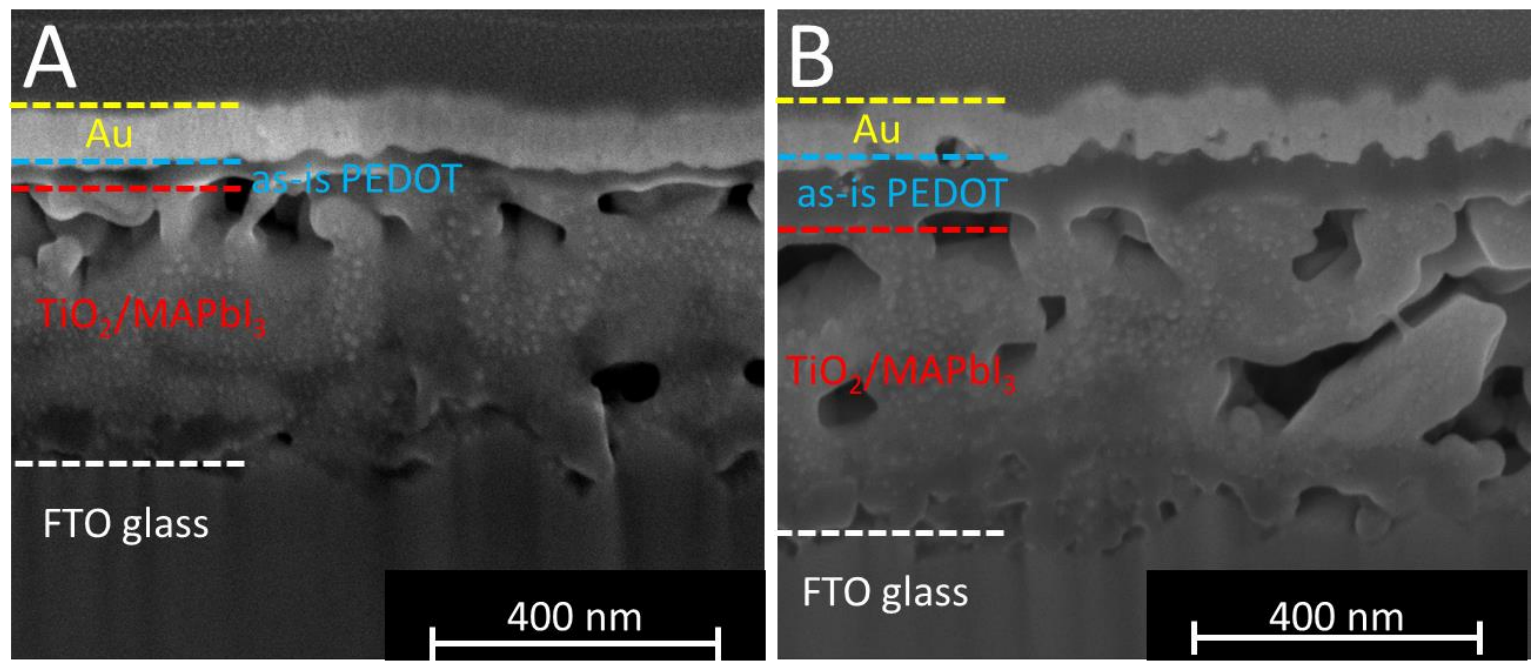

Figure 59. Cross-sectional SEM images of a solar cell, where the PEDOT electrodeposition was carried out at $E=+1.0 \mathrm{~V}$ for $\boldsymbol{A}: Q_{p o l}=5 \mathrm{mC} \mathrm{cm}{ }^{-2}$, and B: $Q_{p o l}=10$ $m C \mathrm{~cm}^{-2}$. In both cases the electrodeposition was immediately followed by a reduction step at $E=-0.6$ for $t=60 \mathrm{~s}$.

\subsubsection{Fine-tuning the Electrochemical Post Treatment}

Three different strategies were employed to mitigate the destruction of the $\mathrm{MAPbI}_{3}$ layer: (i) rapid reduction at $-0.6 \mathrm{~V}$ for $10 \mathrm{~s}$ (Figure 60A); (ii) mild reduction at $-0.5 \mathrm{~V}$ for $60 \mathrm{~s}$ (Figure 60B); and (iii) rapid-mild reduction at $-0.5 \mathrm{~V}$ for $20 \mathrm{~s}$ (Figure 60C). By employing a rapid reduction treatment, the current increase during later stages (between 20 and $60 \mathrm{~s}$ ) of the post treatment was successfully avoided and the magnitude of the reduction current related to the PEDOT layer stayed similar. However, when using milder reduction conditions (Figure 60B and C) only PEDOT reduction occurred and no current flow at later stages of the treatment could be observed. Furthermore, the magnitude of the current related to the PEDOT reduction was reduced, which could indicate that a lesser amount of PEDOT is reduced during milder conditions. 

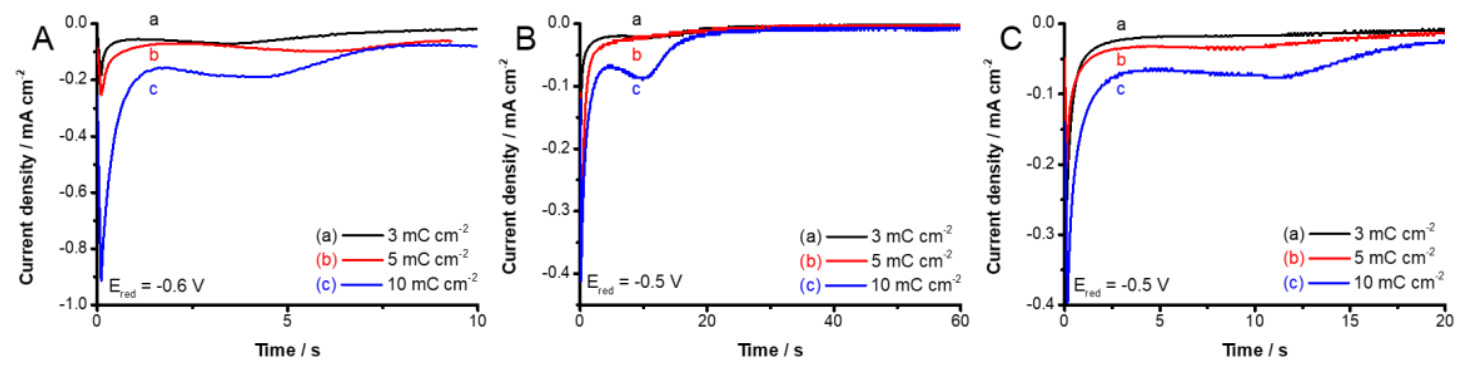

Figure 60. Chronoamperometric curves recorded for the post-treatment of $\mathrm{FTO} / \mathrm{TiO}_{2} / \mathrm{MAPbI}_{3} / \mathrm{PEDOT}$ solar cells, where the PEDOT layers were electrodeposited at $E=+1.0 \mathrm{~V}$. A reductive post treatment of $A$ : rapid reduction at $E=-0.6 \mathrm{~V}$ for $t=10 \mathrm{~s}, \boldsymbol{B}$ : mild reduction at $E=-0.5 \mathrm{~V}$ for $t=60 \mathrm{~s}$, and $\mathrm{C}$ : rapid-mild reduction at $E=-0.5 \mathrm{~V}$ for $t$

$$
=20 \mathrm{~s} \text { was used. }
$$

In all cases, there was an improvement in the $\mathrm{V}_{\mathrm{OC}}$ of the devices compared to the untreated PEDOT (Figure 61). In the case of the rapid reduction (Figure 61A) still significant difference between the $\mathrm{V}_{O C}$ of the devices with different PEDOT thicknesses was observed. The device with a thin $\left(3 \mathrm{mC} \mathrm{cm}^{-2}\right)$ PEDOT coating performed poorly because of the insufficient protection during the electrochemical post-reduction step of the thin PEDOT layer. In the case of the milder reduction treatments the deviation between the $\mathrm{V}_{O C}$ values were close to each other. This can indicate that the milder reduction step leaves more portion of the perovskite layer intact. However, the performances still remained low, because the $\mathrm{J}_{\mathrm{SC}}$ and the FF remained low in most cases. Thus even though the milder reduction step leaves the perovskite layer unharmed it is not sufficient to improve the properties of the PEDOT layer, thus resulting in devices with low efficiencies. The best performing cells were fabricated with the rapid reduction step, where the champion cell had a $5.9 \%$ efficiency (Figure 62).
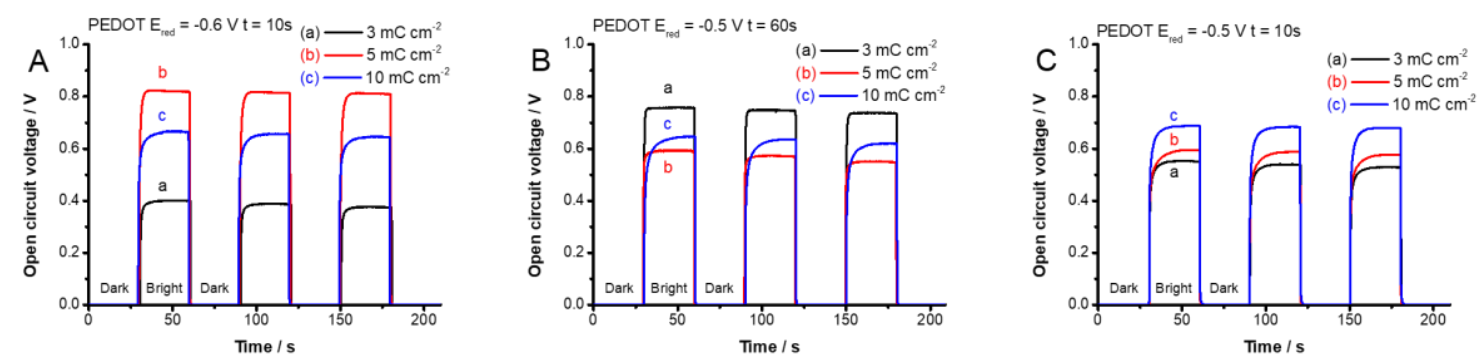

Figure 61. The effect of PEDOT thickness (polymerization charge density) on the open circuit voltage in a $\mathrm{FTO} / \mathrm{TiO}_{2} / \mathrm{MAPbI}_{3} / \mathrm{PEDOT}$ solar cell for PEDOT layers electrodeposited at $E=+1.0 \mathrm{~V}$. The applied post-reduction step was $\mathrm{A}:$ rapid reduction at $E=-0.6$ V for $t=10 \mathrm{~s}, \boldsymbol{B}:$ mild reduction at $E=-0.5 \mathrm{~V}$ for $t=60 \mathrm{~s}$, and $\boldsymbol{C}:$ rapid-mild reduction at $E=-0.5 \mathrm{~V}$ for $t=20 \mathrm{~s}$. 


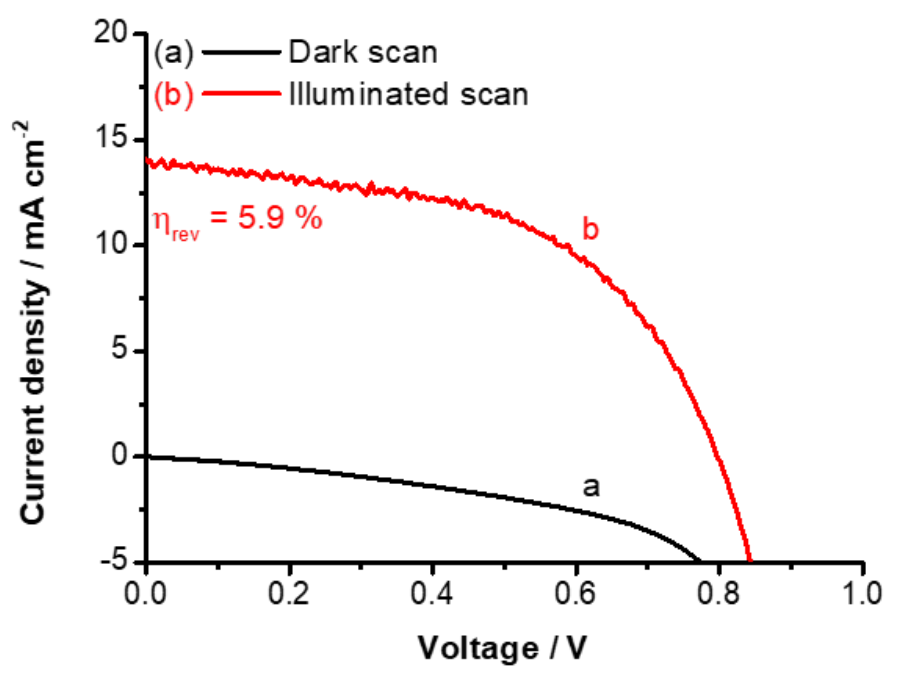

Figure 62. $J$-V curve of the champion device containing a PEDOT layer electrodeposited

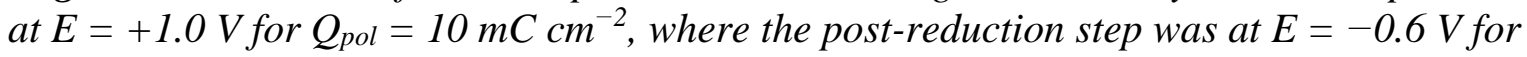
$t=10 \mathrm{~s}$.

\subsubsection{Transient Absorption Spectroscopy of Electrodeposited PEDOT Hole Transporters}

To probe the hole accepting ability of the electrodeposited PEDOT, TAS measurements were carried out (Figure 63A) on the solar cells that are identical to the champion device (PEDOT layer electrodeposited at $\mathrm{E}=+1.0 \mathrm{~V}$ for $\mathrm{Q}_{\mathrm{pol}}=10 \mathrm{mC} \mathrm{cm}^{-2}$, where the post-reduction step was at $\mathrm{E}=-0.6 \mathrm{~V}$ for $\mathrm{t}=10 \mathrm{~s})$. The characteristics of the spectra are in good accordance with $\mathrm{MAPbI}_{3}$ spectra in the literature. ${ }^{[39,94]}$ The most prominent feature, the ground-state bleach at $760 \mathrm{~nm}$, is caused by charge separation due to band edge transition in $\mathrm{MAPbI}_{3}{ }^{[39]}$. Furthermore, there is no additional bleaching signal present at $\sim 500 \mathrm{~nm}$ that would arise from $\mathrm{PbI}_{2}$ in the material. The recovery of the $760 \mathrm{~nm}$ bleach follows second order kinetics (Figure 63B). 

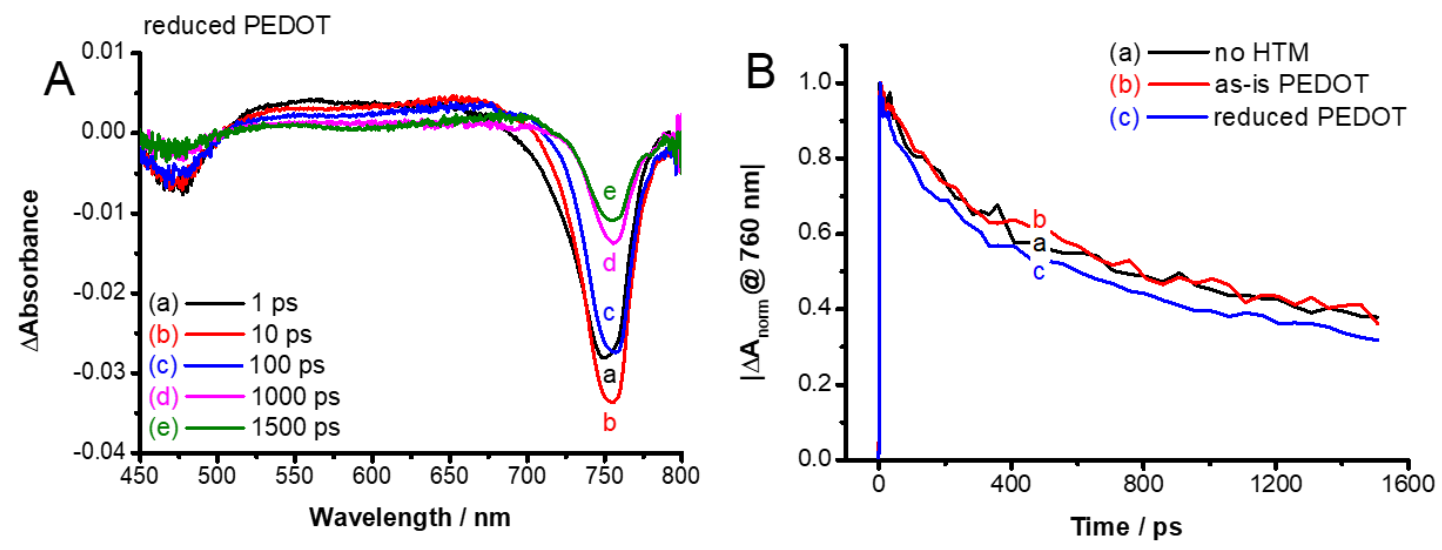

Figure 63. A: Time-resolved transient spectra of an $\mathrm{FTO} / \mathrm{TiO}_{2} / \mathrm{MAPbI}_{3} / \mathrm{PEDOT}$ solar cell employing a thin $\mathrm{MAPbI}_{3}$ layer following $387 \mathrm{~nm}$ laser pulse excitation. The PEDOT layer was prepared and post-treated just as the champion device. B: Bleaching recovery profiles at $760 \mathrm{~nm}$ of different $\mathrm{FTO} / \mathrm{TiO}_{2} / \mathrm{MAPbI}_{3} / \mathrm{PEDOT}$ solar cells.

To calculate the average lifetime two methods can be used. Both methods use the parameters obtained from the biexponential fits of the kinetic traces $\left(A_{i}\right.$ stands for the amplitude and $\tau_{\mathrm{i}}$ stands for the exponent). The exact formula to calculate these, are described by Eq. 15-16:

$$
\begin{gathered}
\tau_{\text {avg. }}=\frac{\sum A_{i} \tau_{i}}{\sum A_{i}} \\
\tau_{\text {weighted avg. }}=\frac{\sum\left(A_{i} \tau_{i}\right)^{2}}{\sum A_{i} \tau_{i}}
\end{gathered}
$$

Both calculation methods yielded similar values within the error of the measurements. The determined values together with the parameters can be found in Table 7. For comparative purposes lifetimes determined by the weighted average method are used.

Table 7. Determined lifetimes, from biexponential fits carried out on TA data. The standard deviation of the measurements was calculated from three separate measurements.

\begin{tabular}{|c|c|c|c|c|c|c|}
\hline Sample & $\mathbf{A}_{1}$ & $\tau_{1} / \mathbf{p s}$ & $\mathbf{A}_{2}$ & $\tau_{2} / \mathbf{p s}$ & $\tau_{\text {avg. }} / \mathbf{p s}$ & $\begin{array}{c}\tau_{\text {weighed avg. }} / \\
\text { ps }\end{array}$ \\
\hline MAPbI $_{3}$ & $0.32 \pm 0.07$ & $200 \pm 54$ & $0.68 \pm 0.07$ & $2600 \pm 440$ & $1873 \pm 240$ & $1747 \pm 173$ \\
\hline $\begin{array}{c}\mathrm{MAPbI}_{3}-\text { as-is } \\
\mathrm{PEDOT}^{\mathrm{MAPb}}-\text { reduced } \\
\text { PEDOT }\end{array}$ & $0.30 \pm 0.02$ & $173 \pm 10$ & $0.70 \pm 0.02$ & $2487 \pm 276$ & $1776 \pm 180$ & $1664 \pm 130$ \\
\hline
\end{tabular}


Fitting the data to a bi-exponential decay reveals that the average lifetime in solar cells with different HTLs has the following trend: reduced PEDOT $\left(\mathrm{t}_{\mathrm{avg}}=1280 \mathrm{ps}\right)<$ as-is PEDOT $\left(\mathrm{t}_{\mathrm{av}}=1660 \mathrm{ps}\right)<$ no HTM $\left(\mathrm{t}_{\mathrm{avg}}=1750 \mathrm{ps}\right)$. This indicates that the PEDOT layer readily accepts holes from $\mathrm{MAPbI}_{3}$ furthermore, reducing the doping level of PEDOT through the post-synthetic reduction technique is a viable strategy to improve the hole accepting properties of the material. 


\section{Summary}

Electrochemistry can be a viable tool to probe the optoelectronic properties of HOIPs. Furthermore, sophisticated electrochemical synthesis techniques are yet to be utilized in device fabrication. A major bottleneck in the application of HOIPs in these electrochemical scenarios is their extreme sensitivity. Our first goal was to establish a guideline on how to perform electrochemical experiments to obtain reliable data. As a next step we built on the gathered experiences and aimed to unravel the effect of the ETL on the charge separation process. For this purpose, a unique coupled technique, ultrafast transient spectroelectrochemistry was used for the first time. To study the interaction of light with mixed-halide PSCs, we investigated the effect of phase segregation of mixed halide solar cells on their electronic properties. We also investigated the possibility of electrosynthesis techniques on the preparation of a PEDOT HTL in $\mathrm{MAPbI}_{3}$ electrodes, thus forming a regular $n-i-p$ junction solar cell.

The most important results of the dissertation can be summarized in the following points:

1. Electrodes were prepared from two different lead halide perovskites $\left(\mathrm{CsPbBr}_{3}\right.$ and $\mathrm{MAPbI}_{3}$ ) by spin-coating method. Steady-state UV-vis measurements were used to evaluate the stability of the perovskite layers in different DCM-based electrolytes. It was found that the speed of dissolution depends on the used anion and follows the following trend: $\mathrm{BF}_{4}^{-}>\mathrm{ClO}_{4}^{-}>>\mathrm{PF}_{6}^{-}$. Thus, electrochemical experiments can be safely performed in $\mathrm{Bu}_{4} \mathrm{NPF}_{6} / \mathrm{DCM}$ electrolyte, because even after 2 hours no sign of substantial degradation was observed. To determine the electrochemical stability window of the layers spectroelectrochemical experiments were carried out. Multiple redox events were observed, and a $-1.0 \mathrm{~V}-+0.6 \mathrm{~V}$ vs $\mathrm{Ag} / \mathrm{AgCl}$ for $\mathrm{CsPbBr}_{3}$ and $-0.65 \mathrm{~V}-+0.55 \mathrm{~V}$ vs $\mathrm{Ag} / \mathrm{AgCl}$ for $\mathrm{MAPbI}_{3}$ stability window was determined.

2. To understand the exact mechanism of the potential induced degradation of the $\mathrm{CsPbBr}_{3}$ layers we investigated the chemical changes during the anodic and cathodic redox waves. XPS measurements revealed that during oxidation the $\mathrm{CsPF}_{6}$ is formed on the electrode surface, parallel to the degradation of the perovskite structure. This degradation is accompanied by a morphological change in the layers. The formation of rectangular particles can be observed on the surface, together with the partial dissolution of the layers (through the leaching of $\mathrm{Pb}^{2+}$ and $\mathrm{Br}^{-}$). During reduction, the formation of $\mathrm{Pb}$ was observed. It was found that $\mathrm{Pb}$ is partially re-oxidized to 
$\mathrm{PbO}$ upon air exposure. The morphology of the electrodes was also heavily impacted, as the degradation was severe enough to expose the underlying $\mathrm{TiO}_{2}$ layer. Furthermore, the formation of dendritic structures was also seen on certain parts of the electrodes. No major change in morphology or composition was observed within the previously proposed stability regime.

3. We combined electrochemistry and TAS to evaluate the effect of external bias on the charge carrier recombination processes. $\mathrm{FTO} / \mathrm{TiO}_{2} / \mathrm{CsPbBr}_{3}$ electrodes were fabricated. At $0.0 \mathrm{~V}$ and more negative potentials the rate constant of charge carrier recombination showed small variation. On the other hand, at more positive potentials, a faster decay kinetics was observed. Since electrons are depleted from the $\mathrm{TiO}_{2}$ layer under anodic bias, photogenerated electrons in $\mathrm{CsPbr}_{3}$ are injected into the $\mathrm{TiO}_{2}$ film. This competing charge injection process resulted in faster bleaching recovery at anodic bias.

4. We unraveled the effect of the substrate (ETL) on the charge recombination processes in $\mathrm{CsPBr}_{3}$ films. In situ ultrafast transient spectroelectrochemical experiments were carried out with $\mathrm{FTO} / \mathrm{CsPbBr}_{3}$ electrodes (i.e., without $\mathrm{TiO}_{2}$ layer). We found that the bleaching recovery was not influenced by the applied electrochemical bias (within the studied time-range). These results further elucidate the role of $\mathrm{TiO}_{2}$ in influencing the charge carrier recombination processes in perovskite films

5. We studied the effect of light-induced phase segregation on the optical and electronic properties of $\mathrm{MAPbBr}_{1.5} \mathrm{I}_{1.5}$ solar cells. Steady state UV-vis absorption spectroscopy was used to evaluate the segregation process. After 30 minutes of white light illumination a decrease in absorbance at $620 \mathrm{~nm}$ was observed which suggests the disappearance of the mixed halide phase. Simultaneously, a concurrent increase in the absorption at shorter and longer wavelength was observed that represents the formation of bromide rich and iodide rich phases. This segregation process has a marked impact on the IPCE of the devices. It was found that inducing segregation a $50 \%$ drop in the IPCE can be observed, which detrimentally affects the performance of such solar cells.

6. When the solar cells are stored in the dark the absorbance completely recovers, within three hours. We found that the recovery of the IPCE is a much slower process and it can take up to 36 hours, but it is almost completely reversible ( 90\% recovery). The slower recovery of the electronic properties suggests that charge 
carrier extraction from the devices is hindered. This was attributed to the lingering effect of the formed residual traps during phase segregation in the devices.

7. Electrochemical polymerization methods were employed to deposit PEDOT HTL on $\mathrm{MAPbI}_{3}$ electrodes, thus forming a regular $n-i-p$ junction solar cell. By using bisEDOT as the monomer, the conservation of the $\mathrm{MAPbI}_{3}$ layer was ensured. Potentiostatic electrodeposition resulted in HTLs with controlled thicknesses. We demonstrated that by controlling the polymerization charge density the thickness of the PEDOT layer can be fine-tuned.

8. To improve the efficiency of the prepared solar cells, electrochemical post-treatment techniques were used. We found that the reduction of the PEDOT layer can improve the $\mathrm{V}_{\mathrm{OC}}$ of the devices. However, this post-reduction step has an unintended side effect, and the $\mathrm{MAPbI}_{3}$ layer can be degraded. Three milder post-reduction techniques were proposed. Because of the milder reduction a champion device with a power conversion efficiency of $5.9 \%$ was achieved. We carried out femtosecond TAS measurements on the champion architecture. These studies revealed that electrodeposited PEDOT is indeed an effective HTM for PSCs. 


\section{6. Összefoglalás}

Az elektrokémiai módszerek egyrészt fontos eszközei lehetnek a hibrid szerves-szervetlen perovszkitok optoelektronikai sajátságainak feltárásához, másrészt e módszerek szintézis céljából történő felhasználása is sok lehetőséget rejt magában. A legnagyobb hátráltató tényező a hibrid szerves-szervetlen perovszkitok környezeti hatásokkal szembeni érzékenysége. Ezért célul tüztük ki, egy olyan körülményrendszer kidolgozását, mely betartása mellett megbízható elektrokémiai mérések végezhetők ezen az érzékeny anyagcsaládon. Ezen tapasztalatokat felhasználva az elektrontranszport-réteg a töltés szétválasztási lépésben betöltött szerepét vizsgáltuk. Ezt egy egyedi csatolt technika fejlesztésével (ultragyors tranziens spektroelektrokémia) hajtottuk végre. Ahhoz, hogy vizsgáljuk a megvilágítás hatását is, tanulmányoztuk a fázisszeparáció hatását vegyes halogenidtartalmú perovszkit napelemek elektronikus sajátságaira. Továbbá elektrokémiai módszerek segítségével PEDOT lyukvezető réteget választottunk le $\mathrm{MAPbI}_{3}$ elektródokra, ezáltal kialakítva n-i-p típusú napelemeket.

A munka tudományos tartalma összefoglalható az alábbi pontokban:

1. A doktori munkám során elvégeztem különböző ólom-halogenid tartalmú perovszkit elektródok $\left(\mathrm{CsPbBr} 3\right.$ és $\left.\mathrm{MAPbI}_{3}\right)$ előállítását forgótárcsás rétegképzés segítségével. UV-látható spektroszkópiás mérések segítségével megvizsgáltuk ezen elektródok stabilitását különböző diklórmetán alapú (DCM) elektrolit oldatokban. Megállapítottuk, hogy a perovszkit rétegek leoldódásának sebessége függ a használt anion minőségétől és az alábbi trendet követi: $\mathrm{BF}_{4}^{-}>\mathrm{ClO}_{4}^{-}>\mathrm{PF}_{6}^{-}$. Így bebizonyítottuk, hogy elektrokémiai mérések biztonsággal végezhetők $\mathrm{Bu}_{4} \mathrm{NPF}_{6} / \mathrm{DCM}$ elektrolitban, ugyanis ebben a közegben 2 óra után sem következik be jelentős degradáció. Ezt követően az elektrokémiai stabilitási potenciáltartomány meghatározását tüztük ki célul, melyet spektroelektrokémiai vizsgálatokkal végeztünk. A vizsgált potenciáltartományban több redoxi eseményt azonosítottunk, és a $\mathrm{CsPbBr} 3$ rétegek esetén a $-1.0 \mathrm{~V}-+0.6 \mathrm{~V}$ vs. $\mathrm{Ag} / \mathrm{AgCl}$ illetve a $\mathrm{MAPbI}_{3}$ rétegek esetén a $-0.65 \mathrm{~V}-+0.55 \mathrm{~V}$ vs. $\mathrm{Ag} / \mathrm{AgCl}$ stabilitási tartományt határoztuk meg.

2. Azért, hogy feltárjuk a potenciál okozta degradáció mechanizmusát, követtük a $\mathrm{CsPbBr}_{3}$ rétegekben bekövetkező kémiai változásokat az anódos és katódos félciklusok során. XPS vizsgálatokkal bebizonyítottuk, hogy a rétegek oxidációja során $\mathrm{CsPF}_{6}$ képződik az elektródok felületén. Ezen felül megállapítottuk, hogy az 
összetételbéli változásokhoz morfológiai változások is társulnak. Az elektródok felületén téglatest alakú részecskék képződését figyeltük meg, miközben a rétegek leoldódását tapasztaltuk $\left(\mathrm{Pb}^{2+}\right.$ és $\mathrm{Br}^{-}$formájában). A rétegek redukciója során fém ólom megjelenését figyeltük meg. $\mathrm{A} \mathrm{Pb}$ egy része viszont $\mathrm{PbO}$-á oxidálódik a levegővel történő érintkezés során. A rétegek morfológiája ebben az esetben is jelentősen változott. A degradáció a redukciós esetben olyan mértékü volt, hogy a $\mathrm{TiO}_{2}$ alapréteg is láthatóvá vált. Ezen felül pedig dendritek megjelenését is tapasztaltuk. Amennyiben viszont a javasolt potenciáltartományon belül végezzük vizsgálatainkat, akkor a rétegek kémiai összetételében és morfológiájában csak csekély változás volt tapasztalható.

3. Miután megállapítottuk a $\mathrm{CsPbBr}_{3}$ elektródokra jellemző elektrokémiai stabilitási potenciáltartományt, megvizsgáltuk a külső potenciál hatását a rétegekben zajló rekombinációs folyamatokra. Ehhez elektrokémiai és tranziens abszorpciós technikák kombinálását valósítottuk meg. $0.0 \mathrm{~V}$-nál negatívabb potenciálokon a töltéshordozók rekombinációjára jellemző sebességi együttható értékében nem tapasztaltunk jelentős változást. Azonban $0.0 \mathrm{~V}$-nál pozitívabb esetekben a folyamat kinetikájának felgyorsulása volt megfigyelhető. Ezt azzal magyaráztuk, hogy anódos potenciál használata esetén a $\mathrm{TiO}_{2}$ réteg elszegényedik elektronokban, ezért a $\mathrm{CsPbBr}_{3}$ rétegekben generált töltéshordozók számára egy új út nyílik meg. Ez a töltés-injektálási folyamat a $\mathrm{TiO}_{2}$ réteg irányába verseng a rekombinációs folyamattal, ezért tapasztaltuk az excitonhoz rendelhető elnyelés gyorsabb visszaállását.

4. Megvizsgáltuk az elektronvezető-réteg hatását a $\mathrm{CsPbBr} 3$ rétegekben bekövetkező rekombinációs folyamatokra. Ehhez $\mathrm{FTO} / \mathrm{Cs} \mathrm{PbBr}_{3}$ rétegeken $\left(\mathrm{a} \mathrm{TiO}_{2}\right.$ alapréteg elhagyásával) ultragyors spektroelektrokémiai vizsgálatokat végeztünk. Ebben az esetben azt állapítottuk meg, hogy az alkalmazott potenciál nincs hatással az excitonokhoz rendelhető elnyelés visszaállásának sebességére (legalábbis az általunk vizsgált időtartományban). Ezek az eredmények további bizonyítékul szolgálnak a $\mathrm{TiO}_{2}$ részvételére a $\mathrm{CsPbBr}_{3}$ rétegekben bekövetkező töltésrekombinációs folyamatokban.

5. Tanulmányoztuk a fény hatására bekövetkező fázisszeparáció hatását a $\mathrm{MAPbBr}_{1.5} \mathrm{I}_{1.5}$ napelemek optikai és elektromos sajátságaira. UV-látható spektrofotometria segítségével vizsgáltuk a fázisszeparációt. 30 perc fehér fénnyel történő megvilágítás után a napelemek fényelnyelésében 620 nm-nél csökkenést 
tapasztaltunk, mely a vegyes halogenid-tartalmú fázis mennyiségének csökkenésével magyarázható. Ezzel egyidőben, rövidebb és hosszabb hullámhosszokon egyaránt abszorbancia növekedést tapasztaltunk, mely a bromid- és jodid-gazdag fázisok megjelenését bizonyítja. Ez a fázisszeparációs folyamat hatással van a napelemek IPCE görbéjére is. A szeparációt követően ugyanis 50 \% csökkenést tapasztaltunk az IPCE értékekben, mely fázisszeparáción átesett napelemek hatékonyságában is megmutatkozik.

6. A napelemek sötétben való tárolását követően, azok optikai sajátságai 3 órát követően teljesen regenerálódnak. Azt tapasztaltuk, hogy az IPCE értékek visszaállása egy sokkal időigényesebb folyamat, ami akár 36 órát is igénybe vehet. Ennek ellenére a folyamat közel reverzibilisnek tekinthető ( $90 \%$-os regenerálódás). Az elektromos sajátságok lassabb visszaállásából azt a következtetést vontuk le, hogy a töltéshordozók kinyerése válik gátolt folyamattá a napelemekben, amely a perovszkit rétegekben kialakuló hibahelyek lassú eltünésével magyarázható.

7. Elektrokémiai polimerizáció segítségével sikeresen választottunk le PEDOT lyukvezető réteget $\mathrm{MAPbI}_{3}$ elektródok felületére, ezzel kialakítva egy $n$-i- $p$ típusú napelemet. $\mathrm{A} \mathrm{MAPbI}_{3}$ réteg degradációjának elkerülése végett, az elektrokémiai leválasztás során bis-EDOT monomert használtunk. Potenciosztatikus leválasztás alkalmazásával sikeresen szabályoztuk a PEDOT lyukvezető réteg vastagságát.

8. Elektrokémiai utókezelések használatával kívántuk a PEDOT lyukvezető réteget tartalmazó napelemek hatékonyságát növelni. A napelemek $V_{O C}$ értékében érdemi javulást tudtunk előidézni a PEDOT rétegek elektroredukciójával. Azonban ennek az utókezelésnek nem kívánt mellékhatásaként a $\mathrm{MAPbI}_{3}$ rétegek degradációját tapasztaltuk. Három enyhébb utóredukciós kezelést vizsgáltunk meg. Ennek köszönhetően a legjobb teljesítményü napelem hatékonysága elérte az 5.8 \%-ot. Femtoszekundumos tranziens abszorpciós spektroszkópia segítségével megállapítottuk, hogy a PEDOT réteg képes hatékony lyukvezető rétegként funkcionálni. 


\section{References}

[1] W.S. Yang, B.-W. Park, E.H. Jung, N.J. Jeon, Y.C. Kim, D.U. Lee, S.S. Shin, J. Seo, E.K. Kim, J.H. Noh, S. Il Seok, Iodide Management in Formamidinium-Lead-Halide-Based Perovskite Layers for Efficient Solar Cells, Science 356 (2017) 1376-1379.

[2] Y. Yang, M. Yang, K. Zhu, J.C. Johnson, J.J. Berry, J. van de Lagemaat, M.C. Beard, Large Polarization-Dependent Exciton Optical Stark Effect in Lead Iodide Perovskites, Nat. Commun. 7 (2016) 12613.

[3] Z.-K. Tan, R.S. Moghaddam, M.L. Lai, P. Docampo, R. Higler, F. Deschler, M. Price, A. Sadhanala, L.M. Pazos, D. Credgington, F. Hanusch, T. Bein, H.J. Snaith, R.H. Friend, Bright Light-Emitting Diodes Based on Organometal Halide Perovskite, Nat. Nanotechnol. 9 (2014) 687-692.

[4] S.A. Veldhuis, P.P. Boix, N. Yantara, M. Li, T.C. Sum, N. Mathews, S.G. Mhaisalkar, Perovskite Materials for Light-Emitting Diodes and Lasers, Adv. Mater. 28 (2016) 6804-6834.

[5] H. Wang, D.H. Kim, Perovskite-Based Photodetectors: Materials and Devices, Chem. Soc. Rev. 46 (2017) 5204-5236.

[6] M. Ahmadi, T. Wu, B. Hu, A Review on Organic-Inorganic Halide Perovskite Photodetectors: Device Engineering and Fundamental Physics, Adv. Mater. 29 (2017) 1605242.

[7] B. Yang, F. Zhang, J. Chen, S. Yang, X. Xia, T. Pullerits, W. Deng, K. Han, Ultrasensitive and Fast All-Inorganic Perovskite-Based Photodetector via Fast Carrier Diffusion, Adv. Mater. 29 (2017) 1703758.

[8] S. Yakunin, M. Sytnyk, D. Kriegner, S. Shrestha, M. Richter, G.J. Matt, H. Azimi, C.J. Brabec, J. Stangl, M. V. Kovalenko, W. Heiss, Detection of X-ray Photons by Solution-Processed Lead Halide Perovskites, Nat. Photonics. 9 (2015) 444-449.

[9] S. Yakunin, D.N. Dirin, Y. Shynkarenko, V. Morad, I. Cherniukh, O. Nazarenko, D. Kreil, T. Nauser, M. V. Kovalenko, Detection of Gamma Photons Using Solution-Grown Single Crystals of Hybrid Lead Halide Perovskites, Nat. Photonics. 10 (2016) 585-589.

[10] V.M. Goldschmidt, Die Gesetze der Krystallochemie, Naturwissenschaften. 14 (1926) 477-485.

[11] A.M. Glazer, The Classification of Tilted Octahedra in Perovskites, Acta Crystallogr. Sect. B Struct. Crystallogr. Cryst. Chem. 28 (1972) 3384-3392.

[12] D.B. Mitzi, Templating and Structural Engineering in Organic-Inorganic Perovskites, J. Chem. Soc. Dalt. Trans. 0 (2001) 1-12.

[13] B. Saparov, D.B. Mitzi, Organic-Inorganic Perovskites: Structural Versatility for Functional Materials Design, Chem. Rev. 116 (2016) 4558-4596.

[14] Y. Chen, Y. Sun, J. Peng, W. Zhang, X. Su, K. Zheng, T. Pullerits, Z. Liang, Tailoring Organic Cation of 2D Air-Stable Organometal Halide Perovskites for Highly Efficient Planar Solar Cells, Adv. Energy Mater. 7 (2017) 1700162.

[15] L. Liang, L. Wencong, C. Nianyi, On the Criteria of Formation and Lattice Distortion of PerovskiteType Complex Halides, J. Phys. Chem. Solids. 65 (2004) 855-860.

[16] C. Li, X. Lu, W. Ding, L. Feng, Y. Gao, Z. Guo, Formability of $A B X_{3}(X=F, C l, B r, I)$ Halide Perovskites, Acta Crystallogr. Sect. B Struct. Sci. 64 (2008) 702-707.

[17] G. Kieslich, S. Sun, A.K. Cheetham, Solid-State Principles Applied to Organic-Inorganic Perovskites: New Tricks for an Old Dog, Chem. Sci. 5 (2014) 4712-4715.

[18] G. Kieslich, S. Sun, A.K. Cheetham, An Extended Tolerance Factor Approach for Organic-Inorganic Perovskites, Chem. Sci. 6 (2015) 3430-3433.

[19] J.S. Manser, J.A. Christians, P. V. Kamat, Intriguing Optoelectronic Properties of Metal Halide Perovskites, Chem. Rev. 116 (2016) 12956-13008.

[20] J.D. Donaldson, J. Silver, S. Hadjiminolis, S.D. Ross, Effects of the Presence of Valence-Shell NonBonding Electron Pairs on the Properties and Structures of Caesium Tin(II) Bromides and of Related Antimony and Tellurium Compounds, J. Chem. Soc. Dalt. Trans. 0 (1975) 1500-1506.

[21] R.J. Worhatch, H. Kim, I.P. Swainson, A.L. Yonkeu, S.J.L. Billinge, Study of Local Structure in Selected Organic-Inorganic Perovskites in the Pm3m Phase, Chem. Mater. 20 (2008) 1272-1277.

[22] I. Swainson, L. Chi, J.-H. Her, L. Cranswick, P. Stephens, B. Winkler, D.J. Wilson, V. Milman, Orientational Ordering, Tilting and Lone-Pair Activity in the Perovskite Methylammonium Tin Bromide, $\mathrm{CH}_{3} \mathrm{NH}_{3} \mathrm{SnBr}_{3}$, Acta Crystallogr. Sect. B Struct. Sci. 66 (2010) $422-429$.

[23] T. Mizokawa, Jahn-Teller Effects in Transition-Metal Compounds with Small Charge-Transfer Energy, J. Phys. Conf. Ser. 428 (2013) 12020.

[24] N. Matsushita, H. Kitagawa, N. Kojima, A Three-Dimensional Iodo-Bridged Mixed-Valence Gold(I, III) Compound, $\mathrm{Cs}_{2} \mathrm{Au}(\mathrm{I}) \mathrm{Au}(\mathrm{III}) I_{6}$, Acta Crystallogr. Sect. C Cryst. Struct. Commun. 53 (1997) 663666. 
[25] N. Elliott, L. Pauling, The Crystal Structure of Cesium Aurous Auric Chloride, Cs ${ }_{2} A u A u C l_{6}$, and Cesium Argentous Auric Chloride, Cs $\mathrm{AgAuCl}_{6}$, J. Am. Chem. Soc. 60 (1938) 1846-1851.

[26] T. Jeon, S.J. Kim, J. Yoon, J. Byun, H.R. Hong, T.-W. Lee, J.-S. Kim, B. Shin, S.O. Kim, Hybrid Perovskites: Effective Crystal Growth for Optoelectronic Applications, Adv. Energy Mater. 7 (2017) 1602596.

[27] H.-B. Kim, H. Choi, J. Jeong, S. Kim, B. Walker, S. Song, J.Y. Kim, Mixed Solvents for the Optimization of Morphology in Solution-Processed, Inverted-Type Perovskite/Fullerene Hybrid Solar Cells, Nanoscale. 6 (2014) 6679-6683.

[28] D.P. Nenon, J.A. Christians, L.M. Wheeler, J.L. Blackburn, E.M. Sanehira, B. Dou, M.L. Olsen, K. Zhu, J.J. Berry, J.M. Luther, Structural and Chemical Evolution of Methylammonium Lead Halide Perovskites During Thermal Processing From Solution, Energy Environ. Sci. 9 (2016) 2072-2082.

[29] J.S. Manser, B. Reid, P. V. Kamat, Evolution of Organic-Inorganic Lead Halide Perovskite from Solid-State Iodoplumbate Complexes, J. Phys. Chem. C. 119 (2015) 17065-17073.

[30] G.E. Eperon, V.M. Burlakov, P. Docampo, A. Goriely, H.J. Snaith, Morphological Control for High Performance, Solution-Processed Planar Heterojunction Perovskite Solar Cells, Adv. Funct. Mater. 24 (2014) 151-157.

[31] G. Grancini, S. Marras, M. Prato, C. Giannini, C. Quarti, F. De Angelis, M. De Bastiani, G.E. Eperon, H.J. Snaith, L. Manna, A. Petrozza, The Impact of the Crystallization Processes on the Structural and Optical Properties of Hybrid Perovskite Films for Photovoltaics, J. Phys. Chem. Lett. 5 (2014) 38363842.

[32] M. Liu, M.B. Johnston, H.J. Snaith, Efficient Planar Heterojunction Perovskite Solar Cells by Vapour Deposition, Nature. 501 (2013) 395-398.

[33] N.J. Jeon, J.H. Noh, Y.C. Kim, W.S. Yang, S. Ryu, S. Il Seok, Solvent Engineering for HighPerformance Inorganic-Organic Hybrid Perovskite Solar Cells, Nat. Mater. 13 (2014) 897-903.

[34] M. Xiao, F. Huang, W. Huang, Y. Dkhissi, Y. Zhu, J. Etheridge, A. Gray-Weale, U. Bach, Y.-B. Cheng, L. Spiccia, A Fast Deposition-Crystallization Procedure for Highly Efficient Lead Iodide Perovskite Thin-Film Solar Cells, Angew. Chemie. 126 (2014) 10056-10061.

[35] S. Paek, P. Schouwink, E.N. Athanasopoulou, K.T. Cho, G. Grancini, Y. Lee, Y. Zhang, F. Stellacci, M.K. Nazeeruddin, P. Gao, From Nano- to Micrometer Scale: The Role of Antisolvent Treatment on High Performance Perovskite Solar Cells, Chem. Mater. 29 (2017) 3490-3498.

[36] H. Yu, F. Wang, F. Xie, W. Li, J. Chen, N. Zhao, The Role of Chlorine in the Formation Process of " $\mathrm{CH}_{3} \mathrm{NH}_{3} \mathrm{PbI}_{3-x} \mathrm{Cl}_{x}$ ” Perovskite, Adv. Funct. Mater. (2014) 7102-7108

[37] S. Colella, E. Mosconi, P. Fedeli, A. Listorti, F. Gazza, F. Orlandi, P. Ferro, T. Besagni, A. Rizzo, G. Calestani, G. Gigli, F. De Angelis, R. Mosca, $M A P b I_{3-x} C l_{x}$ Mixed Halide Perovskite for Hybrid Solar Cells: The Role of Chloride as Dopant on the Transport and Structural Properties, Chem. Mater. 25 (2013) 4613-4618.

[38] B. Li, C. Fei, K. Zheng, X. Qu, T. Pullerits, G. Cao, J. Tian, Constructing Water-Resistant $\mathrm{CH}_{3} \mathrm{NH}_{3} \mathrm{PbI}_{3}$ Perovskite Films via Coordination Interaction, J. Mater. Chem. A. 4 (2016) 17018-17024.

[39] K.G. Stamplecoskie, J.S. Manser, P. V. Kamat, Dual Nature of the Excited State in Organic-Inorganic Lead Halide Perovskites, Energy Environ. Sci. 8 (2015) 208-215.

[40] N. Ahn, D.-Y. Son, I.-H. Jang, S.M. Kang, M. Choi, N.-G. Park, Highly Reproducible Perovskite Solar Cells with Average Efficiency of $18.3 \%$ and Best Efficiency of $19.7 \%$ Fabricated via Lewis Base Adduct of Lead(II) Iodide, J. Am. Chem. Soc. 137 (2015) 8696-8699.

[41] J.S. Manser, M.I. Saidaminov, J.A. Christians, O.M. Bakr, P. V. Kamat, Making and Breaking of Lead Halide Perovskites, Acc. Chem. Res. 49 (2016) 330-338.

[42] Y. Guo, K. Shoyama, W. Sato, Y. Matsuo, K. Inoue, K. Harano, C. Liu, H. Tanaka, E. Nakamura, Chemical Pathways Connecting Lead(II) Iodide and Perovskite via Polymeric Plumbate(II) Fiber, J. Am. Chem. Soc. 137 (2015) 15907-15914.

[43] D.T. Moore, H. Sai, K.W. Tan, D.-M. Smilgies, W. Zhang, H.J. Snaith, U. Wiesner, L.A. Estroff, Crystallization Kinetics of Organic-Inorganic Trihalide Perovskites and the Role of the Lead Anion in Crystal Growth, J. Am. Chem. Soc. 137 (2015) 2350-2358.

[44] K. Liang, D.B. Mitzi, M.T. Prikas, Synthesis and Characterization of Organic-Inorganic Perovskite Thin Films Prepared Using a Versatile Two-Step Dipping Technique, Chem. Mater. 10 (1998) 403411.

[45] J. Burschka, N. Pellet, S.-J. Moon, R. Humphry-Baker, P. Gao, M.K. Nazeeruddin, M. Grätzel, Sequential Deposition as a Route to High-Performance Perovskite-Sensitized Solar Cells, Nature. 499 (2013) 316-319.

[46] Z. Xiao, C. Bi, Y. Shao, Q. Dong, Q. Wang, Y. Yuan, C. Wang, Y. Gao, J. Huang, Efficient, High Yield Perovskite Photovoltaic Devices Grown by Interdiffusion of Solution-Processed Precursor Stacking Layers, Energy Environ. Sci. 7 (2014) 2619-2623. 
[47] L.K. Ono, M.R. Leyden, S. Wang, Y. Qi, Organometal Halide Perovskite Thin Films and Solar Cells by Vapor Deposition, J. Mater. Chem. A. 4 (2016) 6693-6713.

[48] D. Forgács, L. Gil-Escrig, D. Pérez-Del-Rey, C. Momblona, J. Werner, B. Niesen, C. Ballif, M. Sessolo, H.J. Bolink, Efficient Monolithic Perovskite/Perovskite Tandem Solar Cells, Adv. Energy Mater. 7 (2017) 1602121.

[49] D. Shi, V. Adinolfi, R. Comin, M. Yuan, E. Alarousu, A. Buin, Y. Chen, S. Hoogland, A. Rothenberger, K. Katsiev, Y. Losovyj, X. Zhang, P.A. Dowben, O.F. Mohammed, E.H. Sargent, O.M. Bakr, Low Trap-State Density and Long Carrier Diffusion in Organolead Trihalide Perovskite Single Crystals, Science 347 (2015) 519-522.

[50] Y. Dang, Y. Liu, Y. Sun, D. Yuan, X. Liu, W. Lu, G. Liu, H. Xia, X. Tao, Bulk Crystal Growth of Hybrid Perovskite Material $\mathrm{CH}_{3} \mathrm{NH}_{3} \mathrm{PbI}_{3}$, CrystEngComm. 17 (2015) 665-670.

[51] M.I. Saidaminov, A.L. Abdelhady, B. Murali, E. Alarousu, V.M. Burlakov, W. Peng, I. Dursun, L. Wang, Y. He, G. Maculan, A. Goriely, T. Wu, O.F. Mohammed, O.M. Bakr, High-Quality Bulk Hybrid Perovskite Single Crystals Within Minutes by Inverse Temperature Crystallization, Nat. Commun. 6 (2015) 7586.

[52] G. Maculan, A.D. Sheikh, A.L. Abdelhady, M.I. Saidaminov, M.A. Haque, B. Murali, E. Alarousu, O.F. Mohammed, T. Wu, O.M. Bakr, $\mathrm{CH}_{3} \mathrm{NH}_{3} \mathrm{PbCl}_{3}$ Single Crystals: Inverse Temperature Crystallization and Visible-Blind UV-Photodetector, J. Phys. Chem. Lett. 6 (2015) 3781-3786.

[53] M.I. Saidaminov, A.L. Abdelhady, G. Maculan, O.M. Bakr, Retrograde Solubility of Formamidinium and Methylammonium Lead Halide Perovskites Enabling Rapid Single Crystal Growth, Chem. Commun. 51 (2015) 17658-17661.

[54] F. Brivio, K.T. Butler, A. Walsh, M. van Schilfgaarde, Relativistic Quasiparticle Self-Consistent Electronic Structure of Hybrid Halide Perovskite Photovoltaic Absorbers, Phys. Rev. B. 89 (2014) 155204.

[55] P. Umari, E. Mosconi, F. De Angelis, Relativistic GW Calculations on $\mathrm{CH}_{3} \mathrm{NH}_{3} \mathrm{PbI}_{3}$ and $\mathrm{CH}_{3} \mathrm{NH}_{3} \mathrm{SnI}_{3}$ Perovskites for Solar Cell Applications, Sci. Rep. 4 (2015) 4467.

[56] A. Miyata, A. Mitioglu, P. Plochocka, O. Portugall, J.T.-W. Wang, S.D. Stranks, H.J. Snaith, R.J. Nicholas, Direct Measurement of the Exciton Binding Energy and Effective Masses for Charge Carriers in Organic-Inorganic Trihalide Perovskites, Nat. Phys. 11 (2015) 582-587.

[57] J. Huang, Y. Yuan, Y. Shao, Y. Yan, Understanding the Physical Properties of Hybrid Perovskites for Photovoltaic Applications, Nat. Rev. Mater. 2 (2017) 17042.

[58] W.-J. Yin, T. Shi, Y. Yan, Unique Properties of Halide Perovskites as Possible Origins of the Superior Solar Cell Performance, Adv. Mater. 26 (2014) 4653-4658.

[59] N. Jensen, U. Rau, R.M. Hausner, S. Uppal, L. Oberbeck, R.B. Bergmann, J.H. Werner, Recombination Mechanisms in Amorphous Silicon/Crystalline Silicon Heterojunction Solar Cells, J. Appl. Phys. 87 (2000) 2639-2645.

[60] S. De Wolf, J. Holovsky, S.-J. Moon, P. Löper, B. Niesen, M. Ledinsky, F.-J. Haug, J.-H. Yum, C. Ballif, Organometallic Halide Perovskites: Sharp Optical Absorption Edge and Its Relation to Photovoltaic Performance, J. Phys. Chem. Lett. 5 (2014) 1035-1039.

[61] D.P. McMeekin, G. Sadoughi, W. Rehman, G.E. Eperon, M. Saliba, M.T. Horantner, A. Haghighirad, N. Sakai, L. Korte, B. Rech, M.B. Johnston, L.M. Herz, H.J. Snaith, A Mixed-Cation Lead MixedHalide Perovskite Absorber for Tandem Solar Cells, Science 351 (2016) 151-155.

[62] Y. Hu, E.M. Hutter, P. Rieder, I. Grill, J. Hanisch, M.F. Aygüler, A.G. Hufnagel, M. Handloser, T. Bein, A. Hartschuh, K. Tvingstedt, V. Dyakonov, A. Baumann, T.J. Savenije, M.L. Petrus, P. Docampo, Understanding the Role of Cesium and Rubidium Additives in Perovskite Solar Cells: Trap States, Charge Transport, and Recombination, Adv. Energy Mater. (2018) 1703057.

[63] T. Jesper Jacobsson, J.-P. Correa-Baena, M. Pazoki, M. Saliba, K. Schenk, M. Grätzel, A. Hagfeldt, Exploration of the Compositional Space for Mixed Lead Halogen Perovskites for High Efficiency Solar Cells, Energy Environ. Sci. 9 (2016) 1706-1724.

[64] S. Sarkar, P. Mahadevan, Role of the A-site Cation in Determining the Properties of the Hybrid Perovskite $\mathrm{CH}_{3} \mathrm{NH}_{3} \mathrm{PbBr}_{3}$, Phys. Rev. B. 95 (2017) 214118.

[65] C.W. Myung, J. Yun, G. Lee, K.S. Kim, A New Perspective on the Role of A-Site Cations in Perovskite Solar Cells, Adv. Energy Mater. (2018) 1702898.

[66] G. Giorgi, J.-I. Fujisawa, H. Segawa, K. Yamashita, Cation Role in Structural and Electronic Properties of 3D Organic-Inorganic Halide Perovskites: A DFT Analysis, J. Phys. Chem. C. 118 (2014) 12176-12183.

[67] A. Binek, F.C. Hanusch, P. Docampo, T. Bein, Stabilization of the Trigonal High-Temperature Phase of Formamidinium Lead Iodide, J. Phys. Chem. Lett. 6 (2015) 1249-1253.

[68] Z. Li, M. Yang, J.-S. Park, S.-H. Wei, J.J. Berry, K. Zhu, Stabilizing Perovskite Structures by Tuning Tolerance Factor: Formation of Formamidinium and Cesium Lead Iodide Solid-State Alloys, Chem. 
Mater. 28 (2016) 284-292.

[69] P. Kanhere, S. Chakraborty, C.J. Rupp, R. Ahuja, Z. Chen, Substitution Induced Band Structure Shape Tuning in Hybrid Perovskites $\left(\mathrm{CH}_{3} \mathrm{NH}_{3} \mathrm{~Pb}_{1-x} \mathrm{Sn}_{x} \mathrm{I}_{3}\right)$ for Efficient Solar Cell Applications, RSC Adv. 5 (2015) 107497-107502.

[70] Y. Ogomi, A. Morita, S. Tsukamoto, T. Saitho, N. Fujikawa, Q. Shen, T. Toyoda, K. Yoshino, S.S. Pandey, T. Ma, S. Hayase, $\mathrm{CH}_{3} \mathrm{NH}_{3} \mathrm{Sn}_{x} \mathrm{~Pb}_{(1-x)} I_{3}$ Perovskite Solar Cells Covering up to $1060 \mathrm{~nm}, \mathrm{~J}$. Phys. Chem. Lett. 5 (2014) 1004-1011.

[71] F. Hao, C.C. Stoumpos, R.P.H. Chang, M.G. Kanatzidis, Anomalous Band Gap Behavior in Mixed Sn and $\mathrm{Pb}$ Perovskites Enables Broadening of Absorption Spectrum in Solar Cells, J. Am. Chem. Soc. 136 (2014) 8094-8099.

[72] G. Nedelcu, L. Protesescu, S. Yakunin, M.I. Bodnarchuk, M.J. Grotevent, M. V. Kovalenko, Fast Anion-Exchange in Highly Luminescent Nanocrystals of Cesium Lead Halide Perovskites $\left(C_{s} P b X_{3}, X\right.$ $=C l, B r, I)$, Nano Lett. 15 (2015) 5635-5640.

[73] Q.A. Akkerman, V. D’Innocenzo, S. Accornero, A. Scarpellini, A. Petrozza, M. Prato, L. Manna, Tuning the Optical Properties of Cesium Lead Halide Perovskite Nanocrystals by Anion Exchange Reactions, J. Am. Chem. Soc. 137 (2015) 10276-10281.

[74] G. Li, J.Y.-L. Ho, M. Wong, H.S. Kwok, Reversible Anion Exchange Reaction in Solid Halide Perovskites and Its Implication in Photovoltaics, J. Phys. Chem. C. 119 (2015) 26883-26888.

[75] D.M. Jang, K. Park, D.H. Kim, J. Park, F. Shojaei, H.S. Kang, J.-P. Ahn, J.W. Lee, J.K. Song, Reversible Halide Exchange Reaction of Organometal Trihalide Perovskite Colloidal Nanocrystals for Full-Range Band Gap Tuning, Nano Lett. 15 (2015) 5191-5199.

[76] N. Pellet, J. Teuscher, J. Maier, M. Grätzel, Transforming Hybrid Organic Inorganic Perovskites by Rapid Halide Exchange, Chem. Mater. 27 (2015) 2181-2188.

[77] J.H. Noh, S.H. Im, J.H. Heo, T.N. Mandal, S. Il Seok, Chemical Management for Colorful, Efficient, and Stable Inorganic-Organic Hybrid Nanostructured Solar Cells, Nano Lett. 13 (2013) 1764-1769.

[78] J.B. Hoffman, A.L. Schleper, P. V. Kamat, Transformation of Sintered CsPbBr 3 Nanocrystals to Cubic $\mathrm{CsPbI}_{3}$ and Gradient CsPbBr$I_{3-x}$ through Halide Exchange, J. Am. Chem. Soc. 138 (2016) 86038611.

[79] M.C. Brennan, S. Draguta, P. V. Kamat, M. Kuno, Light-Induced Anion Phase Segregation in Mixed Halide Perovskites, ACS Energy Lett. 3 (2018) 204-213.

[80] E.T. Hoke, D.J. Slotcavage, E.R. Dohner, A.R. Bowring, H.I. Karunadasa, M.D. McGehee, Reversible Photo-Induced Trap Formation in Mixed-Halide Hybrid Perovskites for Photovoltaics, Chem. Sci. 6 (2015) 613-617.

[81] Q. Dong, Y. Fang, Y. Shao, P. Mulligan, J. Qiu, L. Cao, J. Huang, Electron-Hole Diffusion Lengths > 175 um in Solution-Grown $\mathrm{CH}_{3} \mathrm{NH}_{3} \mathrm{PbI}_{3}$ Single Crystals, Science 347 (2015) 967-970.

[82] A. Kojima, K. Teshima, Y. Shirai, T. Miyasaka, Organometal Halide Perovskites as Visible-Light Sensitizers for Photovoltaic Cells, J. Am. Chem. Soc. 131 (2009) 6050-6051.

[83] J.-H. Im, C.-R. Lee, J.-W. Lee, S.-W. Park, N.-G. Park, $6.5 \%$ Efficient Perovskite Quantum-DotSensitized Solar Cell, Nanoscale. 3 (2011) 4088-4093.

[84] J.M. Ball, M.M. Lee, A. Hey, H.J. Snaith, Low-Temperature Processed Meso-Superstructured to ThinFilm Perovskite Solar Cells, Energy Environ. Sci. 6 (2013) 1739-1743.

[85] Y. Li, S. Ye, W. Sun, W. Yan, Y. Li, Z. Bian, Z. Liu, S. Wang, C. Huang, Hole-Conductor-Free Planar Perovskite Solar Cells with 16.0 \% Efficiency, J. Mater. Chem. A. 3 (2015) 18389-18394.

[86] J. Shi, J. Dong, S. Lv, Y. Xu, L. Zhu, J. Xiao, X. Xu, H. Wu, D. Li, Y. Luo, Q. Meng, Hole-ConductorFree Perovskite Organic Lead Iodide Heterojunction Thin-Film Solar Cells: High Efficiency and Junction Property, Appl. Phys. Lett. 104 (2014) 63901.

[87] V. D’Innocenzo, G. Grancini, M.J.P. Alcocer, A.R.S. Kandada, S.D. Stranks, M.M. Lee, G. Lanzani, H.J. Snaith, A. Petrozza, Excitons Versus Free Charges in Organo-Lead Trihalide Perovskites, Nat. Commun. 5 (2014) 1-6.

[88] K. Tanaka, T. Takahashi, T. Ban, T. Kondo, K. Uchida, N. Miura, Comparative Study on the Excitons in Lead-Halide-Based Perovskite-Type Crystals $\mathrm{CH}_{3} \mathrm{NH}_{3} \mathrm{PbBr}_{3} \mathrm{CH}_{3} \mathrm{NH}_{3} \mathrm{PbI}_{3}$, Solid State Commun. 127 (2003) 619-623.

[89] M. Hu, C. Bi, Y. Yuan, Z. Xiao, Q. Dong, Y. Shao, J. Huang, Distinct Exciton Dissociation Behavior of Organolead Trihalide Perovskite and Excitonic Semiconductors Studied in the Same System, Small. 11 (2015) 2164-2169.

[90] T.C. Sum, N. Mathews, Advancements in Perovskite Solar Cells: Photophysics Behind the Photovoltaics, Energy Environ. Sci. 7 (2014) 2518-2534.

[91] G. Xing, N. Mathews, S. Sun, S.S. Lim, Y.M. Lam, M. Gratzel, S. Mhaisalkar, T.C. Sum, Long-Range Balanced Electron- and Hole-Transport Lengths in Organic-Inorganic $\mathrm{CH}_{3} \mathrm{NH}_{3} \mathrm{PbI}_{3}$, Science 342 (2013) 344-347. 
[92] K. Zheng, Q. Zhu, M. Abdellah, M.E. Messing, W. Zhang, A. Generalov, Y. Niu, L. Ribaud, S.E. Canton, T. Pullerits, Exciton Binding Energy and the Nature of Emissive States in Organometal Halide Perovskites, J. Phys. Chem. Lett. 6 (2015) 2969-2975.

[93] S.D. Stranks, G.E. Eperon, G. Grancini, C. Menelaou, M.J.P. Alcocer, T. Leijtens, L.M. Herz, A. Petrozza, H.J. Snaith, Electron-Hole Diffusion Lengths Exceeding 1 Micrometer in an Organometal Trihalide Perovskite Absorber, Science 342 (2013) 341-344.

[94] J. Peng, Y. Chen, K. Zheng, T. Pullerits, Z. Liang, Insights Into Charge Carrier Dynamics in OrganoMetal Halide Perovskites: From Neat Films to Solar Cells, Chem. Soc. Rev. 46 (2017) 5714-5729.

[95] K. Zheng, K. Žídek, M. Abdellah, J. Chen, P. Chábera, W. Zhang, M.J. Al-Marri, T. Pullerits, High Excitation Intensity Opens a New Trapping Channel in Organic-Inorganic Hybrid Perovskite Nanoparticles, ACS Energy Lett. 1 (2016) 1154-1161.

[96] J. Chen, P. Chábera, T. Pascher, M.E. Messing, R. Schaller, S. Canton, K. Zheng, T. Pullerits, Enhanced Size Selection in Two-Photon Excitation for CsPbBr 3 Perovskite Nanocrystals, J. Phys. Chem. Lett. 8 (2017) 5119-5124.

[97] H. Huang, M.I. Bodnarchuk, S. V. Kershaw, M. V. Kovalenko, A.L. Rogach, Lead Halide Perovskite Nanocrystals in the Research Spotlight: Stability and Defect Tolerance, ACS Energy Lett. 2 (2017) 2071-2083.

[98] K. Zheng, K. Žídek, M. Abdellah, M.E. Messing, M.J. Al-Marri, T. Pullerits, Trap States and Their Dynamics in Organometal Halide Perovskite Nanoparticles and Bulk Crystals, J. Phys. Chem. C. 120 (2016) 3077-3084.

[99] J.M. Ball, A. Petrozza, Defects in Perovskite-Halides and Their Effects in Solar Cells, Nat. Energy. 1 (2016) 16149.

[100] D. Macdonald, A. Cuevas, Understanding Carrier Trapping in Multicrystalline Silicon, Sol. Energy Mater. Sol. Cells. 65 (2001) 509-516.

[101] P. Parkinson, J. Lloyd-Hughes, Q. Gao, H.H. Tan, C. Jagadish, M.B. Johnston, L.M. Herz, Transient Terahertz Conductivity of GaAs Nanowires, Nano Lett. 7 (2007) 2162-2165.

[102] P. Sasanpour, R. Mohammadpour, Trap Density Measurement in Three-Dimensional Nanostructured Photoelectrode of Titanium Dioxide with Various Morphologies, Appl. Phys. A. 122 (2016) 476.

[103] Y. Fang, Q. Dong, Y. Shao, Y. Yuan, J. Huang, Highly Narrowband Perovskite Single-Crystal Photodetectors Enabled by Surface-Charge Recombination, Nat. Photonics. 9 (2015) 679-686.

[104] S.Y. Leblebici, L. Leppert, Y. Li, S.E. Reyes-Lillo, S. Wickenburg, E. Wong, J. Lee, M. Melli, D. Ziegler, D.K. Angell, D.F. Ogletree, P.D. Ashby, F.M. Toma, J.B. Neaton, I.D. Sharp, A. WeberBargioni, Facet-dependent Photovoltaic Efficiency Variations in Single Grains of Hybrid Halide Perovskite, Nat. Energy. 1 (2016) 16093.

[105] Y. Shao, Z. Xiao, C. Bi, Y. Yuan, J. Huang, Origin and Elimination of Photocurrent Hysteresis by Fullerene Passivation in $\mathrm{CH}_{3} \mathrm{NH}_{3} \mathrm{PbI}_{3}$ Planar Heterojunction Solar Cells, Nat. Commun. 5 (2014) 5784.

[106] J.A. Christians, P.A. Miranda Herrera, P. V. Kamat, Transformation of the Excited State and Photovoltaic Efficiency of $\mathrm{CH}_{3} \mathrm{NH}_{3} \mathrm{PbI}_{3}$ Perovskite upon Controlled Exposure to Humidified Air, J. Am. Chem. Soc. 137 (2015) 1530-1538.

[107] J. Yang, B.D. Siempelkamp, D. Liu, T.L. Kelly, Investigation of $\mathrm{CH}_{3} \mathrm{NH}_{3} \mathrm{PbI}_{3}$ Degradation Rates and Mechanisms in Controlled Humidity Environments Using in Situ Techniques, ACS Nano. 9 (2015) 1955-1963.

[108] A.M.A. Leguy, Y. Hu, M. Campoy-Quiles, M.I. Alonso, O.J. Weber, P. Azarhoosh, M. van Schilfgaarde, M.T. Weller, T. Bein, J. Nelson, P. Docampo, P.R.F. Barnes, Reversible Hydration of $\mathrm{CH}_{3} \mathrm{NH}_{3} \mathrm{PbI}_{3}$ in Films, Single Crystals, and Solar Cells, Chem. Mater. 27 (2015) 3397-3407.

[109] T.A. Berhe, W.-N. Su, C.-H. Chen, C.-J. Pan, J.-H. Cheng, H.-M. Chen, M.-C. Tsai, L.-Y. Chen, A.A. Dubale, B.-J. Hwang, Organometal Halide Perovskite Solar Cells: Degradation and Stability, Energy Environ. Sci. 9 (2016) 323-356.

[110] W. Huang, J.S. Manser, P. V. Kamat, S. Ptasinska, Evolution of Chemical Composition, Morphology, and Photovoltaic Efficiency of $\mathrm{CH}_{3} \mathrm{NH}_{3} \mathrm{PbI}_{3}$ Perovskite under Ambient Conditions, Chem. Mater. 28 (2016) 303-311.

[111] Y. Zhao, K. Zhu, Optical Bleaching of Perovskite $\left(\mathrm{CH}_{3} \mathrm{NH}_{3}\right) \mathrm{PbI}_{3}$ Through Room-Temperature Phase Transformation Induced by Ammonia, Chem. Commun. 50 (2014) 1605-1607.

[112] N. Aristidou, I. Sanchez-Molina, T. Chotchuangchutchaval, M. Brown, L. Martinez, T. Rath, S.A. Haque, The Role of Oxygen in the Degradation of Methylammonium Lead Trihalide Perovskite Photoactive Layers, Angew. Chemie Int. Ed. 54 (2015) 8208-8212.

[113] D. Bryant, N. Aristidou, S. Pont, I. Sanchez-Molina, T. Chotchunangatchaval, S. Wheeler, J.R. Durrant, S.A. Haque, Light and Oxygen Induced Degradation Limits the Operational Stability of Methylammonium Lead Triiodide Perovskite Solar Cells, Energy Environ. Sci. 9 (2016) 1655-1660. 
[114] F.T.F. O’Mahony, Y.H. Lee, C. Jellett, S. Dmitrov, D.T.J. Bryant, J.R. Durrant, B.C. O’Regan, M. Graetzel, M.K. Nazeeruddin, S.A. Haque, Improved Environmental Stability of Organic Lead Trihalide Perovskite-Based Photoactive-Layers in the Presence of Mesoporous $\mathrm{TiO}_{2}$, J. Mater. Chem. A. 3 (2015) 7219-7223.

[115] J. Yang, T.L. Kelly, Decomposition and Cell Failure Mechanisms in Lead Halide Perovskite Solar Cells, Inorg. Chem. 56 (2017) 92-101.

[116] C.C. Stoumpos, C.D. Malliakas, M.G. Kanatzidis, Semiconducting Tin and Lead Iodide Perovskites with Organic Cations: Phase Transitions, High Mobilities, and Near-Infrared Photoluminescent Properties, Inorg. Chem. 52 (2013) 9019-9038.

[117] J.H. Heo, S.H. Im, J.H. Noh, T.N. Mandal, C.-S. Lim, J.A. Chang, Y.H. Lee, H. Kim, A. Sarkar, M.K. Nazeeruddin, M. Grätzel, S. Il Seok, Efficient Inorganic-Organic Hybrid Heterojunction Solar Cells Containing Perovskite Compound and Polymeric Hole Conductors, Nat. Photonics. 7 (2013) 486-491.

[118] J. Padchasri, R. Yimnirun, Effects of Annealing Temperature on Stability of Methylammonium Lead Iodide Perovskite Powders, J. Alloys Compd. 720 (2017) 63-69.

[119] T. Baikie, Y. Fang, J.M. Kadro, M. Schreyer, F. Wei, S.G. Mhaisalkar, M. Graetzel, T.J. White, Synthesis and Crystal Chemistry of the Hybrid Perovskite $\left(\mathrm{CH}_{3} \mathrm{NH}_{3}\right) \mathrm{PbI}_{3}$ for Solid-State Sensitised Solar Cell Applications, J. Mater. Chem. A. 1 (2013) 5628-5641.

[120] S. Aharon, A. Dymshits, A. Rotem, L. Etgar, Temperature Dependence of Hole Conductor Free Formamidinium Lead Iodide Perovskite Based Solar Cells, J. Mater. Chem. A. 3 (2015) 9171-9178.

[121] T. Leijtens, G.E. Eperon, S. Pathak, A. Abate, M.M. Lee, H.J. Snaith, Overcoming Ultraviolet Light Instability of Sensitized $\mathrm{TiO}_{2}$ with Meso-Superstructured Organometal Trihalide Perovskite Solar Cells, Nat. Commun. 4 (2013) 2885.

[122] C.D. Bailie, E.L. Unger, S.M. Zakeeruddin, M. Grätzel, M.D. McGehee, Melt-Infiltration of SpiroOMeTAD and Thermal Instability of Solid-State Dye-Sensitized Solar Cells, Phys. Chem. Chem. Phys. 16 (2014) 4864-4870.

[123] Y. Kato, L.K. Ono, M. V. Lee, S. Wang, S.R. Raga, Y. Qi, Silver Iodide Formation in Methyl Ammonium Lead Iodide Perovskite Solar Cells with Silver Top Electrodes, Adv. Mater. Interfaces. 2 (2015) 1500195.

[124] A. Guerrero, J. You, C. Aranda, Y.S. Kang, G. Garcia-Belmonte, H. Zhou, J. Bisquert, Y. Yang, Interfacial Degradation of Planar Lead Halide Perovskite Solar Cells, ACS Nano. 10 (2016) 218224.

[125] D. Wei, T. Wang, J. Ji, M. Li, P. Cui, Y. Li, G. Li, J.M. Mbengue, D. Song, Photo-induced Degradation of Lead Halide Perovskite Solar Cells Caused by the Hole Transport Layer/Metal Electrode Interface, J. Mater. Chem. A. 4 (2016) 1991-1998.

[126] J. Mizusaki, K. Arai, K. Fueki, Ionic Conduction of the Perovskite-Type Halides, Solid State Ionics. 11 (1983) 203-211.

[127] Y. Yuan, J. Huang, Ion Migration in Organometal Trihalide Perovskite and Its Impact on Photovoltaic Efficiency and Stability, Acc. Chem. Res. 49 (2016) 286-293.

[128] Y. Yuan, J. Chae, Y. Shao, Q. Wang, Z. Xiao, A. Centrone, J. Huang, Photovoltaic Switching Mechanism in Lateral Structure Hybrid Perovskite Solar Cells, Adv. Energy Mater. 5 (2015) 1500615.

[129] Z. Xiao, Y. Yuan, Y. Shao, Q. Wang, Q. Dong, C. Bi, P. Sharma, A. Gruverman, J. Huang, Giant Switchable Photovoltaic Effect in Organometal Trihalide Perovskite Devices, Nat. Mater. 14 (2015) 193-198.

[130] J.L. Minns, P. Zajdel, D. Chernyshov, W. van Beek, M.A. Green, Structure and Interstitial Iodide Migration in Hybrid Perovskite Methylammonium Lead Iodide, Nat. Commun. 8 (2017) 15152.

[131] T.-Y. Yang, G. Gregori, N. Pellet, M. Grätzel, J. Maier, The Significance of Ion Conduction in a Hybrid Organic-Inorganic Lead-Iodide-Based Perovskite Photosensitizer, Angew. Chemie Int. Ed. 54 (2015) 7905-7910.

[132] Y. Yuan, Q. Wang, Y. Shao, H. Lu, T. Li, A. Gruverman, J. Huang, Electric-Field-Driven Reversible Conversion Between Methylammonium Lead Triiodide Perovskites and Lead Iodide at Elevated Temperatures, Adv. Energy Mater. 6 (2016) 1501803.

[133] J. Xing, Q. Wang, Q. Dong, Y. Yuan, Y. Fang, J. Huang, Ultrafast Ion Migration in Hybrid Perovskite Polycrystalline Thin Films Under Light and Suppression in Single Crystals, Phys. Chem. Chem. Phys. 18 (2016) 30484-30490.

[134] M.L. Petrus, J. Schlipf, C. Li, T.P. Gujar, N. Giesbrecht, P. Müller-Buschbaum, M. Thelakkat, T. Bein, S. Hüttner, P. Docampo, Capturing the Sun: A Review of the Challenges and Perspectives of Perovskite Solar Cells, Adv. Energy Mater. 7 (2017) 1700264.

[135] F. Huang, L. Jiang, A.R. Pascoe, Y. Yan, U. Bach, L. Spiccia, Y.-B. Cheng, Fatigue Behavior of Planar $\mathrm{CH}_{3} \mathrm{NH}_{3} \mathrm{PbI}_{3}$ Perovskite Solar Cells Revealed by Light On/Off Diurnal Cycling, Nano Energy. 27 (2016) 509-514. 
[136] W. Nie, J.-C. Blancon, A.J. Neukirch, K. Appavoo, H. Tsai, M. Chhowalla, M.A. Alam, M.Y. Sfeir, C. Katan, J. Even, S. Tretiak, J.J. Crochet, G. Gupta, A.D. Mohite, Light-Activated Photocurrent Degradation and Self-Healing in Perovskite Solar Cells, Nat. Commun. 7 (2016) 11574.

[137] S. Ghosh, S.K. Pal, K.J. Karki, T. Pullerits, Ion Migration Heals Trapping Centers in $\mathrm{CH}_{3} \mathrm{NH}_{3} \mathrm{PbBr}_{3}$ Perovskite, ACS Energy Lett. 2 (2017) 2133-2139.

[138] H.J. Snaith, A. Abate, J.M. Ball, G.E. Eperon, T. Leijtens, N.K. Noel, S.D. Stranks, J.T.-W. Wang, K. Wojciechowski, W. Zhang, Anomalous Hysteresis in Perovskite Solar Cells, J. Phys. Chem. Lett. 5 (2014) 1511-1515.

[139] N. Klein-Kedem, D. Cahen, G. Hodes, Effects of Light and Electron Beam Irradiation on Halide Perovskites and Their Solar Cells, Acc. Chem. Res. 49 (2016) 347-354.

[140] D.J. Slotcavage, H.I. Karunadasa, M.D. McGehee, Light-Induced Phase Segregation in HalidePerovskite Absorbers, ACS Energy Lett. 1 (2016) 1199-1205.

[141] E. Mosconi, F. De Angelis, Mobile Ions in Organohalide Perovskites: Interplay of Electronic Structure and Dynamics, ACS Energy Lett. 1 (2016) 182-188.

[142] C. Eames, J.M. Frost, P.R.F. Barnes, B.C. O’Regan, A. Walsh, M.S. Islam, Ionic Transport in Hybrid Lead Iodide Perovskite Solar Cells, Nat. Commun. 6 (2015) 7497.

[143] A.J. Barker, A. Sadhanala, F. Deschler, M. Gandini, S.P. Senanayak, P.M. Pearce, E. Mosconi, A.J. Pearson, Y. Wu, A.R. Srimath Kandada, T. Leijtens, F. De Angelis, S.E. Dutton, A. Petrozza, R.H. Friend, Defect-Assisted Photoinduced Halide Segregation in Mixed-Halide Perovskite Thin Films, ACS Energy Lett. 2 (2017) 1416-1424.

[144] J. Hu, R. Gottesman, L. Gouda, A. Kama, M. Priel, S. Tirosh, J. Bisquert, A. Zaban, Photovoltage Behavior in Perovskite Solar Cells under Light-Soaking Showing Photoinduced Interfacial Changes, ACS Energy Lett. 2 (2017) 950-956.

[145] F. Brivio, C. Caetano, A. Walsh, Thermodynamic Origin of Photoinstability in the $\left.\mathrm{CH}_{3} \mathrm{NH}_{3} \mathrm{~Pb}_{\left(\mathrm{I}_{1-x}\right.} \mathrm{Br}_{x}\right)_{3}$ Hybrid Halide Perovskite Alloy, J. Phys. Chem. Lett. 7 (2016) 1083-1087.

[146] O. Hentz, Z. Zhao, S. Gradečak, Impacts of Ion Segregation on Local Optical Properties in Mixed Halide Perovskite Films, Nano Lett. 16 (2016) 1485-1490.

[147] S.J. Yoon, S. Draguta, J.S. Manser, O. Sharia, W.F. Schneider, M. Kuno, P. V. Kamat, Tracking Iodide and Bromide Ion Segregation in Mixed Halide Lead Perovskites during Photoirradiation, ACS Energy Lett. 1 (2016) 290-296.

[148] S.J. Yoon, M. Kuno, P. V. Kamat, Shift Happens . How Halide Ion Defects Influence Photoinduced Segregation in Mixed Halide Perovskites, ACS Energy Lett. 2 (2017) 1507-1514.

[149] C.G. Bischak, C.L. Hetherington, H. Wu, S. Aloni, D.F. Ogletree, D.T. Limmer, N.S. Ginsberg, Origin of Reversible Photoinduced Phase Separation in Hybrid Perovskites, Nano Lett. 17 (2017) 1028-1033.

[150] S.J. Yoon, K.G. Stamplecoskie, P. V. Kamat, How Lead Halide Complex Chemistry Dictates the Composition of Mixed Halide Perovskites, J. Phys. Chem. Lett. 7 (2016) 1368-1373.

[151] C.G. Poll, G.W. Nelson, D.M. Pickup, A. V. Chadwick, D.J. Riley, D.J. Payne, Electrochemical Recycling of Lead From Hybrid Organic-Inorganic Perovskites Using Deep Eutectic Solvents, Green Chem. 18 (2016) 2946-2955.

[152] H.-Y. Hsu, L. Ji, H.S. Ahn, J. Zhao, E.T. Yu, A.J. Bard, A Liquid Junction Photoelectrochemical Solar Cell Based on p-Type $\mathrm{MeNH}_{3} \mathrm{PbI}_{3}$ Perovskite with $1.05 \mathrm{~V}$ Open-Circuit Photovoltage, J. Am. Chem. Soc. 137 (2015) 14758-14764.

[153] Z. Li, C.C. Mercado, M. Yang, E. Palay, K. Zhu, Electrochemical Impedance Analysis of PerovskiteElectrolyte Interfaces, Chem. Commun. 53 (2017) 2467-2470.

[154] R.C. Shallcross, Y. Zheng, S.S. Saavedra, N.R. Armstrong, Determining Band-Edge Energies and Morphology-Dependent Stability of Formamidinium Lead Perovskite Films Using Spectroelectrochemistry and Photoelectron Spectroscopy, J. Am. Chem. Soc. 139 (2017) 4866-4878.

[155] V.K. Ravi, G.B. Markad, A. Nag, Band Edge Energies and Excitonic Transition Probabilities of Colloidal CsPbX $\mathrm{S}_{3}(\mathrm{X}=\mathrm{Cl}, \mathrm{Br}, \mathrm{I})$ Perovskite Nanocrystals, ACS Energy Lett. 1 (2016) 665-671.

[156] H.-Y. Hsu, L. Ji, M. Du, J. Zhao, E.T. Yu, A.J. Bard, Optimization of PbI $\mathrm{PMAPbI}_{3}$ Perovskite Composites by Scanning Electrochemical Microscopy, J. Phys. Chem. C. 120 (2016) 19890-19895.

[157] H.-Y. Hsu, L. Ji, M. Du, J. Zhao, E.T. Yu, A.J. Bard, Optimization of Lead-free Organic-inorganic Tin(II) Halide Perovskite Semiconductors by Scanning Electrochemical Microscopy, Electrochim. Acta. 220 (2016) 205-210.

[158] M. Crespo-Quesada, L.M. Pazos-Outón, J. Warnan, M.F. Kuehnel, R.H. Friend, E. Reisner, MetalEncapsulated Organolead Halide Perovskite Photocathode for Solar-Driven Hydrogen Evolution in Water, Nat. Commun. 7 (2016) 12555.

[159] C. Janáky, K. Rajeshwar, Current Trends in Semiconductor Photoelectrochemistry, ACS Energy Lett. 2 (2017) 1425-1428.

[160] N. Vicente, G. Garcia-Belmonte, Organohalide Perovskites are Fast Ionic Conductors, Adv. Energy 
Mater. 7 (2017) 1700710.

[161] H.-R. Xia, W.-T. Sun, L.-M. Peng, Hydrothermal Synthesis of Organometal Halide Perovskites for Liion Batteries, Chem. Commun. 51 (2015) 13787-13790.

[162] J.A. Dawson, A.J. Naylor, C. Eames, M. Roberts, W. Zhang, H.J. Snaith, P.G. Bruce, M.S. Islam, Mechanisms of Lithium Intercalation and Conversion Processes in Organic-Inorganic Halide Perovskites, ACS Energy Lett. 2 (2017) 1818-1824.

[163] M. Tathavadekar, S. Krishnamurthy, A. Banerjee, S. Nagane, Y. Gawli, A. Suryawanshi, S. Bhat, D. Puthusseri, A.D. Mohite, S. Ogale, Low-Dimensional Hybrid Perovskites as High Performance Anodes for Alkali-Ion Batteries, J. Mater. Chem. A. 5 (2017) 18634-18642.

[164] N. Vicente, G. Garcia-Belmonte, Methylammonium Lead Bromide Perovskite Battery Anodes Reversibly Host High Li-Ion Concentrations, J. Phys. Chem. Lett. 8 (2017) 1371-1374.

[165] M. Hasan, S. Venkatesan, D. Lyashenko, J.D. Slinker, A. Zakhidov, Solvent Toolkit for Electrochemical Characterization of Hybrid Perovskite Films, Anal. Chem. 89 (2017) 9649-9653.

[166] Q. Jiang, M. Chen, J. Li, M. Wang, X. Zeng, T. Besara, J. Lu, Y. Xin, X. Shan, B. Pan, C. Wang, S. Lin, T. Siegrist, Q. Xiao, Z. Yu, Electrochemical Doping of Halide Perovskites with Ion Intercalation, ACS Nano. 11 (2017) 1073-1079.

[167] Q. Jiang, X. Zeng, N. Wang, Z. Xiao, Z. Guo, J. Lu, Electrochemical Lithium Doping Induced Property Changes In Halide Perovskite CsPbBr 3 Crystal, ACS Energy Lett. 3 (2018) 264-269.

[168] Y. Huang, M. Fang, G. Zou, B. Zhang, H. Wang, Monochromatic and Electrochemically Switchable Electrochemiluminescence of Perovskite CsPbBr 3 Nanocrystals, Nanoscale. 8 (2016) 18734-18739.

[169] J. Xue, Z. Zhang, F. Zheng, Q. Xu, J. Xu, G. Zou, L. Li, J.-J. Zhu, Efficient Solid-State Electrochemiluminescence from High-Quality Perovskite Quantum Dot Films, Anal. Chem. 89 (2017) 8212-8216.

[170] X. Tan, B. Zhang, G. Zou, Electrochemistry and Electrochemiluminescence of Organometal Halide Perovskite Nanocrystals in Aqueous Medium, J. Am. Chem. Soc. 139 (2017) 8772-8776.

[171] Y. Huang, X. Long, D. Shen, G. Zou, B. Zhang, H. Wang, Hydrogen Peroxide Involved Anodic Charge Transfer and Electrochemiluminescence of All-Inorganic Halide Perovskite CsPbBr 3 Nanocrystals in an Aqueous Medium, Inorg. Chem. 56 (2017) 10135-10138.

[172] Z. Cai, F. Li, W. Xu, S. Xia, J. Zeng, S. He, X. Chen, Colloidal CsPbBr, Perovskite Nanocrystal Films as Electrochemiluminescence Emitters in Aqueous Solutions, Nano Res. 11 (2018) 1447-1455.

[173] J.A. Christians, P. Schulz, J.S. Tinkham, T.H. Schloemer, S.P. Harvey, B.J. Tremolet de Villers, A. Sellinger, J.J. Berry, J.M. Luther, Tailored Interfaces of Unencapsulated Perovskite Solar Cells for > 1,000 Hour Operational Stability, Nat. Energy. 3 (2018) 68-74.

[174] E. Bi, H. Chen, F. Xie, Y. Wu, W. Chen, Y. Su, A. Islam, M. Grätzel, X. Yang, L. Han, Diffusion Engineering of Ions and Charge Carriers for Stable Efficient Perovskite Solar Cells, Nat. Commun. 8 (2017) 15330.

[175] G. Grancini, C. Roldán-Carmona, I. Zimmermann, E. Mosconi, X. Lee, D. Martineau, S. Narbey, F. Oswald, F. De Angelis, M. Graetzel, M.K. Nazeeruddin, One-Year Stable Perovskite Solar Cells by 2D/3D Interface Engineering, Nat. Commun. 8 (2017) 15684.

[176] H.J. Snaith, Perovskites: The Emergence of a New Era for Low-Cost, High-Efficiency Solar Cells, J. Phys. Chem. Lett. 4 (2013) 3623-3630.

[177] B. O'Regan, M. Grätzel, A Low-Cost, High-Efficiency Solar Cell Based on Dye-Sensitized Colloidal $\mathrm{TiO}_{2}$ Films, Nature. 353 (1991) 737-740.

[178] H.J. Snaith, L. Schmidt-Mende, Advances in Liquid-Electrolyte and Solid-State Dye-Sensitized Solar Cells, Adv. Mater. 19 (2007) 3187-3200.

[179] M. Grätzel, U. Bach, D. Lupo, P. Comte, J.E. Moser, F. Weissörtel, J. Salbeck, H. Spreitzer, SolidState Dye-Sensitized Mesoporous $\mathrm{TiO}_{2}$ Solar Cells with High Photon-to-Electron Conversion Efficiencies, Nature. 395 (1998) 583-585.

[180] M.M. Lee, J. Teuscher, T. Miyasaka, T.N. Murakami, H.J. Snaith, Efficient Hybrid Solar Cells Based on Meso-Superstructured Organometal Halide Perovskites, Science 338 (2012) 643-647.

[181] H.-S. Kim, C.-R. Lee, J.-H. Im, K.-B. Lee, T. Moehl, A. Marchioro, S.-J. Moon, R. Humphry-Baker, J.-H. Yum, J.E. Moser, M. Grätzel, N.-G. Park, Lead Iodide Perovskite Sensitized All-Solid-State Submicron Thin Film Mesoscopic Solar Cell with Efficiency Exceeding 9 \%, Sci. Rep. 2 (2012) 591.

[182] T. Leijtens, S.D. Stranks, G.E. Eperon, R. Lindblad, E.M.J. Johansson, I.J. McPherson, H. Rensmo, J.M. Ball, M.M. Lee, H.J. Snaith, Electronic Properties of Meso-Superstructured and Planar Organometal Halide Perovskite Films: Charge Trapping, Photodoping, and Carrier Mobility, ACS Nano. 8 (2014) 7147-7155.

[183] L. Etgar, P. Gao, Z. Xue, Q. Peng, A.K. Chandiran, B. Liu, M.K. Nazeeruddin, M. Grätzel, Mesoscopic $\mathrm{CH}_{3} \mathrm{NH}_{3} \mathrm{PbI}_{3} / \mathrm{TiO}_{2}$ Heterojunction Solar Cells, J. Am. Chem. Soc. 134 (2012) 17396-17399.

[184] T. Liu, K. Chen, Q. Hu, R. Zhu, Q. Gong, Inverted Perovskite Solar Cells: Progresses and 
Perspectives, Adv. Energy Mater. 6 (2016) 1600457.

[185] S. Ye, H. Rao, Z. Zhao, L. Zhang, H. Bao, W. Sun, Y. Li, F. Gu, J. Wang, Z. Liu, Z. Bian, C. Huang, A Breakthrough Efficiency of 19.9\% Obtained in Inverted Perovskite Solar Cells by Using an Efficient Trap State Passivator Cu(thiourea)I, J. Am. Chem. Soc. 139 (2017) 7504-7512.

[186] M.F. Mohamad Noh, C.H. Teh, R. Daik, E.L. Lim, C.C. Yap, M.A. Ibrahim, N. Ahmad Ludin, A.R. bin Mohd Yusoff, J. Jang, M.A. Mat Teridi, The Architecture of the Electron Transport Layer for a Perovskite Solar Cell, J. Mater. Chem. C. 6 (2018) 682-712.

[187] K. Mahmood, S. Sarwar, M.T. Mehran, Current Status of Electron Transport Layers in Perovskite Solar Cells: Materials and Properties, RSC Adv. 7 (2017) 17044-17062.

[188] Z.H. Bakr, Q. Wali, A. Fakharuddin, L. Schmidt-Mende, T.M. Brown, R. Jose, Advances in Hole Transport Materials Engineering for Stable and Efficient Perovskite Solar Cells, Nano Energy. 34 (2017) 271-305.

[189] S.F. Völker, S. Collavini, J.L. Delgado, Organic Charge Carriers for Perovskite Solar Cells, ChemSusChem. 8 (2015) 3012-3028.

[190] S. Ameen, M.A. Rub, S.A. Kosa, K.A. Alamry, M.S. Akhtar, H.-S. Shin, H.-K. Seo, A.M. Asiri, M.K. Nazeeruddin, Perovskite Solar Cells: Influence of Hole Transporting Materials on Power Conversion Efficiency, ChemSusChem. 9 (2016) 10-27.

[191] Y. Zhang, W. Liu, F. Tan, Y. Gu, The Essential Role of the Poly(3-hexylthiophene) Hole Transport Layer in Perovskite Solar Cells, J. Power Sources. 274 (2015) 1224-1230.

[192] F. Di Giacomo, S. Razza, F. Matteocci, A. D’Epifanio, S. Licoccia, T.M. Brown, A. Di Carlo, High Efficiency $\mathrm{CH}_{3} \mathrm{NH}_{3} \mathrm{PbI}_{(3-x)} \mathrm{Cl}_{x}$ Perovskite Solar Cells with Poly(3-hexylthiophene) Hole Transport Layer, J. Power Sources. 251 (2014) 152-156.

[193] P. Docampo, J.M. Ball, M. Darwich, G.E. Eperon, H.J. Snaith, Efficient Organometal Trihalide Perovskite Planar-Heterojunction Solar Cells on Flexible Polymer Substrates, Nat. Commun. 4 (2013) 2761.

[194] D. Koushik, W.J.H. Verhees, D. Zhang, Y. Kuang, S. Veenstra, M. Creatore, R.E.I. Schropp, Atomic Layer Deposition Enabled Perovskite/PEDOT Solar Cells in a Regular n-i-p Architectural Design, Adv. Mater. Interfaces. 4 (2017) 1700043.

[195] J. Liu, S. Pathak, T. Stergiopoulos, T. Leijtens, K. Wojciechowski, S. Schumann, N. Kausch-Busies, H.J. Snaith, Employing PEDOT as the p-Type Charge Collection Layer in Regular Organic-Inorganic Perovskite Solar Cells, J. Phys. Chem. Lett. 6 (2015) 1666-1673.

[196] X. Jiang, Z. Yu, Y. Zhang, J. Lai, J. Li, G.G. Gurzadyan, X. Yang, L. Sun, High-Performance Regular Perovskite Solar Cells Employing Low-Cost Poly(ethylenedioxythiophene) as a Hole-Transporting Material, Sci. Rep. 7 (2017) 42564.

[197] C. Janáky, K. Rajeshwar, The Role of (Photo)Electrochemistry in the Rational Design of Hybrid Conducting Polymer/Semiconductor Assemblies: From Fundamental Concepts to Practical Applications, Prog. Polym. Sci. 43 (2015) 96-135.

[198] K.L. Gardner, J.G. Tait, T. Merckx, W. Qiu, U.W. Paetzold, L. Kootstra, M. Jaysankar, R. Gehlhaar, D. Cheyns, P. Heremans, J. Poortmans, Nonhazardous Solvent Systems for Processing Perovskite Photovoltaics, Adv. Energy Mater. 6 (2016) 1600386.

[199] N.T. Shewmon, H. Yu, I. Constantinou, E. Klump, F. So, Formation of Perovskite Heterostructures by Ion Exchange, ACS Appl. Mater. Interfaces. 8 (2016) 33273-33279.

[200] G.A. Sotzing, J.R. Reynolds, P.J. Steel, Poly(3,4-ethylenedioxythiophene) (PEDOT) Prepared via Electrochemical Polymerization of EDOT, 2,2'-Bis(3,4-ethylenedioxythiophene) (BiEDOT), and Their TMS Derivatives, Adv. Mater. 9 (1997) 795-798.

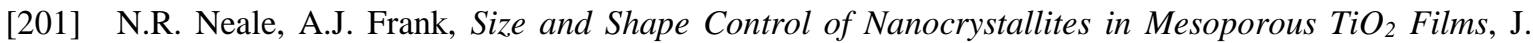
Mater. Chem. 17 (2007) 3216-3221.

[202] J.A. Christians, J.S. Manser, P. V. Kamat, Best Practices in Perovskite Solar Cell Efficiency Measurements. Avoiding the Error of Making Bad Cells Look Good, J. Phys. Chem. Lett. 6 (2015) 852-857.

[203] S.H. Strauss, The Search for Larger and More Weakly Coordinating Anions, Chem. Rev. 93 (1993) 927-942.

[204] I. Krossing, I. Raabe, Noncoordinating Anions - Fact or Fiction? A Survey of Likely Candidates, Angew. Chemie - Int. Ed. 43 (2004) 2066-2090.

[205] Y. Tian, I.G. Scheblykin, Artifacts in Absorption Measurements of Organometal Halide Perovskite Materials: What Are the Real Spectra?, J. Phys. Chem. Lett. 6 (2015) 3466-3470.

[206] S. Sawatani, S. Ogawa, T. Yoshida, H. Minoura, Formation of Highly Crystallized B-PbO Thin Films by Cathodic Electrodeposition of Pb and Its Rapid Oxidation in Air, Adv. Funct. Mater. 15 (2005) 297-302.

[207] Q.A. Akkerman, M. Gandini, F. Di Stasio, P. Rastogi, F. Palazon, G. Bertoni, J.M. Ball, M. Prato, A. 
Petrozza, L. Manna, Strongly Emissive Perovskite Nanocrystal Inks for High-Voltage Solar Cells, Nat. Energy. 2 (2016) 16194.

[208] P. Schulz, E. Edri, S. Kirmayer, G. Hodes, D. Cahen, A. Kahn, Interface Energetics in Organo-Metal Halide Perovskite-Based Photovoltaic Cells, Energy Environ. Sci. 7 (2014) 1377-1381.

[209] H.-Y. Hsu, L. Ji, H.S. Ahn, J. Zhao, E.T. Yu, A.J. Bard, A Liquid Junction Photoelectrochemical Solar Cell Based on p-Type $\mathrm{MeNH}_{3} \mathrm{PbI}_{3}$ Perovskite with $1.05 \mathrm{~V}$ Open-Circuit Photovoltage, J. Am. Chem. Soc. 137 (2015) 14758-14764.

[210] C.M. Cardona, W. Li, A.E. Kaifer, D. Stockdale, G.C. Bazan, Electrochemical Considerations for Determining Absolute Frontier Orbital Energy Levels of Conjugated Polymers for Solar Cell Applications, Adv. Mater. 23 (2011) 2367-2371.

[211] E. Bandiello, J. Ávila, L. Gil-Escrig, E. Tekelenburg, M. Sessolo, H.J. Bolink, Influence of Mobile Ions on the Electroluminescence Characteristics of Methylammonium Lead Iodide Perovskite Diodes, J. Mater. Chem. A. 4 (2016) 18614-18620.

[212] J.S. Manser, P. V. Kamat, Band Filling with Free Charge Carriers in Organometal Halide Perovskites, Nat. Photonics. 8 (2014) 737-743.

[213] C.S. Ponseca, T.J. Savenije, M. Abdellah, K. Zheng, A. Yartsev, T. Pascher, T. Harlang, P. Chabera, T. Pullerits, A. Stepanov, J.-P. Wolf, V. Sundström, Organometal Halide Perovskite Solar Cell Materials Rationalized: Ultrafast Charge Generation, High and Microsecond-Long Balanced Mobilities, and Slow Recombination, J. Am. Chem. Soc. 136 (2014) 5189-5192.

[214] B.C. O’Regan, P.R.F. Barnes, X. Li, C. Law, E. Palomares, J.M. Marin-Beloqui, Optoelectronic Studies of Methylammonium Lead Iodide Perovskite Solar Cells with Mesoporous $\mathrm{TiO}_{2}$ : Separation of Electronic and Chemical Charge Storage, Understanding Two Recombination Lifetimes, and the Evolution of Band Offsets during j-V Hysteresis, J. Am. Chem. Soc. 137 (2015) 5087-5099.

[215] J. Chen, K. Žídek, P. Chábera, D. Liu, P. Cheng, L. Nuuttila, M.J. Al-Marri, H. Lehtivuori, M.E. Messing, K. Han, K. Zheng, T. Pullerits, Size- and Wavelength-Dependent Two-Photon Absorption Cross-Section of $\mathrm{CsPbBr}_{3}$ Perovskite Quantum Dots, J. Phys. Chem. Lett. 8 (2017) 2316-2321.

[216] S. Draguta, S. Thakur, Y. V. Morozov, Y. Wang, J.S. Manser, P. V. Kamat, M. Kuno, Spatially NonUniform Trap State Densities in Solution-Processed Hybrid Perovskite Thin Films, J. Phys. Chem. Lett. 7 (2016) 715-721.

[217] P. V. Kamat, I. Bedja, S. Hotchandani, L.K. Patterson, Photosensitization of Nanocrystalline Semiconductor Films. Modulation of Electron Transfer between Excited Ruthenium Complex and $\mathrm{SnO}_{2}$ Nanocrystallites with an Externally Applied Bias, J. Phys. Chem. 100 (1996) 4900-4908.

[218] Y. Tachibana, S.A. Haque, I.P. Mercer, J.E. Moser, D.R. Klug, J.R. Durrant, Modulation of the Rate of Electron Injection in Dye-Sensitized Nanocrystalline $\mathrm{TiO}_{2}$ Films by Externally Applied Bias, J. Phys. Chem. B. 105 (2001) 7424-7431.

[219] G. Redmond, D. Fitzmaurice, Spectroscopic Determination of Flatband Potentials for Polycrystalline Titania Electrodes in Nonaqueous Solvents, J. Phys. Chem. 97 (1993) 1426-1430.

[220] I. Bedja, S. Hotchandani, P. V. Kamat, Preparation and Photoelectrochemical Characterization of Thin $\mathrm{SnO}_{2}$ Nanocrystalline Semiconductor Films and Their Sensitization with Bis(2,2'bipyridine)(2,2'-bipyridine-4,4'-dicarboxylic acid)ruthenium(II) Complex, J. Phys. Chem. 98 (1994) 4133-4140.

[221] E.H. Anaraki, A. Kermanpur, L. Steier, K. Domanski, T. Matsui, W. Tress, M. Saliba, A. Abate, M. Grätzel, A. Hagfeldt, J.-P. Correa-Baena, Highly Efficient and Stable Planar Perovskite Solar Cells by Solution-Processed Tin Oxide, Energy Environ. Sci. 9 (2016) 3128-3134.

[222] Y. Fang, C. Bi, D. Wang, J. Huang, The Functions of Fullerenes in Hybrid Perovskite Solar Cells, ACS Energy Lett. 2 (2017) 782-794.

[223] S.S. Shin, E.J. Yeom, W.S. Yang, S. Hur, M.G. Kim, J. Im, J. Seo, J.H. Noh, S. Il Seok, Colloidally Prepared La-doped $\mathrm{BaSnO}_{3}$ Electrodes for Efficient, Photostable Perovskite Solar Cells, Science 356 (2017) 167-171.

[224] G.A. Nemnes, C. Besleaga, V. Stancu, D.E. Dogaru, L.N. Leonat, L. Pintilie, K. Torfason, M. Ilkov, A. Manolescu, I. Pintilie, Normal and Inverted Hysteresis in Perovskite Solar Cells, J. Phys. Chem. C. 121 (2017) 11207-11214.

[225] G.F. Samu, C. Visy, K. Rajeshwar, S. Sarker, V.R. Subramanian, C. Janáky, Photoelectrochemical Infiltration of a Conducting Polymer (PEDOT) into Metal-Chalcogenide Decorated $\mathrm{TiO}_{2} \mathrm{Nanotube}$ Arrays, Electrochim. Acta. 151 (2015) 467-476.

[226] H. Gustafsson, C. Kvarnström, A. Ivaska, Comparative Study of n-doping and p-doping of Poly(3,4ethylenedioxythiophene) Electrosynthesised on Aluminium, Thin Solid Films. 517 (2008) 474-478. 


\section{Acknowledgements}

"Smooth Seas Never Made A Skilled Sailor"

Many individuals from different countries took part in the completion of this dissertation through valuable discussions, guidance, or emotional support.

I am grateful to Prof. Ágota Tóth, head of the Department of Physical Chemistry and Materials Science for the opportunity to conduct research and to finish my doctoral studies at the department.

I would like to thank my supervisors, who shaped my thinking and attitude throughout the years. They were the ones who guided me and helped me become the person I am today. I would like to thank Prof. Csaba Visy for his constant support, especially in my early years, when I needed it the most. I am thankful to Dr. Csaba Janáky for his scientific and personal advices. I am grateful for the constant support that allowed me to widen my perspectives and learn how to think critically. I feel fortunate that I could be a part of the excitement that is to found a new research group. During my doctoral studies I had the opportunity to visit two research groups in the USA. I would like to thank my "unofficial" supervisors Prof. Krishnan Rajeshwar and Prof. Prashant Kamat. The time I spent in their laboratories taught me how to be independent and that there is always one more experiment (especially on the day of departure). I was also reminded that the spark of joy should always be with you when doing research.

I would like to thank Dr. Tatyana Orlova and Dr. Gary Zaiats for taking the SEM-FIB images, Steven M. Kobosko for recording the XP spectra, and Dr. Allen G. Oliver for recording the XRD patterns.

I am grateful for the support of my friends and colleagues: Ágnes Dancs, Attila Kormányos, Balázs Endrődi, Jacob Hoffmann, Rebecca Scheidt, Gábor Schuszter, Tamás Boldizsár. Your invigorating presence kept me always motivated.

My deepest gratitude goes to my family: my parents and my sister. The atmosphere you created at home was always a sanctuary for me. It helped me take a breath and continue working towards my goals. 